Doctor Thesis

\title{
Recent Measurement of \\ Flavor Asymmetry of Antiquarks in the Proton by Drell-Yan Experiment SeaQuest at Fermilab
}

\author{
Kei Nagai \\ Department of Physics, Tokyo Institute of Technology
}

February 27, 2017 


\begin{abstract}
A measurement of the flavor asymmetry of the antiquarks $(\bar{d}$ and $\bar{u})$ in the proton is described in this thesis. The proton consists of three valence quarks, sea quarks, and gluons. Antiquarks in the proton are sea quarks. They are generated from the gluon splitting: $g \rightarrow q+\bar{q}$. According to QCD (Quantum Chromodynamics), the gluon splitting is independent of quark flavor. It suggests that the amounts of $\bar{d}$ and $\bar{u}$ should be the same in the proton.
\end{abstract}

However, the NMC experiment at CERN found that the amount of $\bar{d}$ is larger than that of $\bar{u}$ in the proton using the deep inelastic scattering in 1991. This result is obtained for $\bar{d}$ and $\bar{u}$ integrated over Bjorken $x$. Bjorken $x$ is the fraction of the momentum of the parton to that of the proton. The NA51 experiment $(x \sim 0.2)$ at CERN and E866/NuSea experiment $(0.015<x<0.35)$ at Fermilab measured the flavor asymmetry of the antiquarks $(\bar{d} / \bar{u})$ in the proton as a function of $x$ using Drell-Yan process. The experiments reported that the flavor symmetry is broken over all measured $x$ values.

Understanding the flavor asymmetry of the antiquarks in the proton is a challenge of the QCD. The theoretical investigation from the first principle of QCD such as lattice QCD calculation is important. In addition, the QCD effective models and hadron models such as the meson cloud model can also be tested with the flavor asymmetry of antiquarks. From the experimental side, it is important to measure with higher accuracy and in a wider $x$ range. The SeaQuest (E906) experiment measures $\bar{d} / \bar{u}$ at large $x(0.15<x<0.45)$ accurately to understand its behavior.

The SeaQuest experiment is a Drell-Yan experiment at Fermi National Accelerator Laboratory (Fermilab). In the Drell-Yan process of proton-proton reaction, an antiquark in a proton and a quark in another proton annihilate and create a virtual photon, which then decays into a muon pair $\left(q \bar{q} \rightarrow \gamma^{*} \rightarrow \mu^{+} \mu^{-}\right)$. The SeaQuest experiment uses a $120 \mathrm{GeV}$ proton beam extracted from Fermilab's Main Injector. The proton beam interacts with hydrogen and deuterium targets. The SeaQuest spectrometer detects the muon pairs from the Drell-Yan process. The $\bar{d} / \bar{u}$ ratio at $0.1<x<0.58$ is extracted from the number of detected Drell-Yan muon pairs.

After the detector construction, commissioning run and detector upgrade, the SeaQuest experiment started the physics data acquisition from 2013. We finished so far three periods of physics data acquisition. The fourth period is in progress.

The detector construction, detector performance evaluation, data taking and data analysis for the flavor asymmetry of the antiquarks $\bar{d} / \bar{u}$ in the proton are my contribution to SeaQuest.

The cross section ratio of Drell-Yan process in $p-p$ and $p-d$ reactions is obtained from dimuon yields. In the experiment with high beam intensity, it is important to control the tracking efficiency of charged particles through the magnetic spectrometer. The tracking efficiency depends on the chamber occupancy, and the appropriate method for the correction is important. The chamber occupancy is the number of hits in drift chambers. A new method of the correction for the tracking efficiency is developed based on the occupancy, and applied to the data. This method reflects the real response of the drift chambers. Therefore, the systematic error is well controlled by this method.

The flavor asymmetry of antiquarks is obtained at $0.1<x<0.58$. At $0.1<x<0.45$, the result is $\bar{d} / \bar{u}>1$. The result at $0.1<x<0.24$ agrees with the E866 result. The result at $x>0.24$, however, disagrees with the E866 result. The result at $0.45<x<0.58$ is consistent with unity within the statistical errors. 
The $\bar{d} / \bar{u}$ results extracted from experiments are used to investigate the validity of the theoretical models. The present experimental result provides the data points in wide $x$ region. It is useful for understanding the proton structure in the light of QCD and effective hadron models. The present result has a practical application as well. Antiquark distributions are important as inputs to simulations of hadron reactions such as $W^{ \pm}$production in various experiments. The new knowledge on antiquark distributions helps to improve the precision of the simulations. 


\section{Contents}

1 Introduction $\quad 4$

2 Nucleon Structure $\quad 6$

2.1 Deep Inelastic Scattering . . . . . . . . . . . . . . . . . . . 6

2.2 Quantum Chromodynamics (QCD) . . . . . . . . . . . . . . . 8

2.3 Drell-Yan Process . . . . . . . . . . . . . . . . . . . . . . . . 9

2.4 Motivation for the SeaQuest Experiment . . . . . . . . . . . . . . . 12

$2.4 .1 \quad$ NMC Experiment . . . . . . . . . . . . . . . . . . . . . . 12

2.4 .2 NA51 Experiment . . . . . . . . . . . . . . . . . 14

2.4 .3 E866/NuSea Experiment . . . . . . . . . . . . . . . . . 14

2.4.4 Motivation of SeaQuest Experiment . . . . . . . . . . . . 17

3 SeaQuest Experiment $\quad 18$

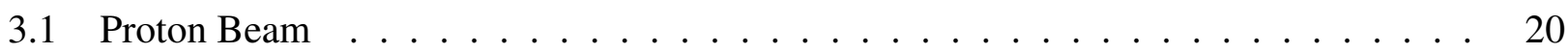

3.2 Beam Monitor . . . . . . . . . . . . . . . . . . . . . . 21

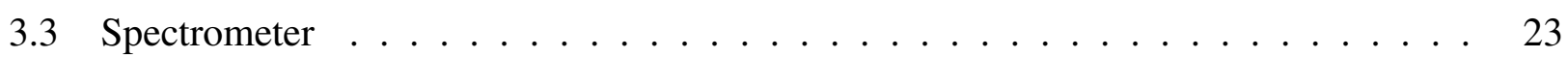

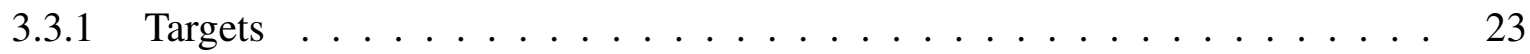

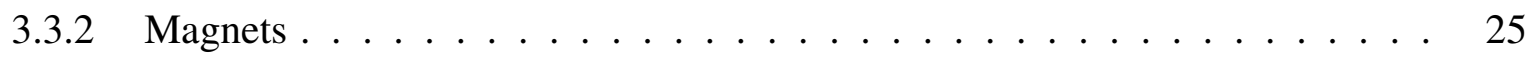

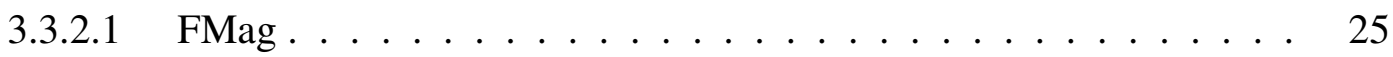

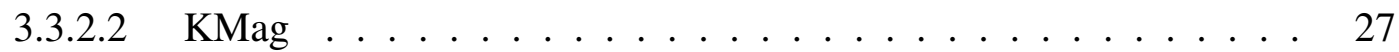

3.3.3 Tracking Stations . . . . . . . . . . . . . . . . . . . 29

3.3.3.1 Hodoscopes . . . . . . . . . . . . . . . . 29

3.3.3.2 Drift Chambers . . . . . . . . . . . . 35

3.3.3.3 Proportional Tubes . . . . . . . . . . . . . . . . . 46

3.3.3.4 Readout System . . . . . . . . . . . . . . . . . 46

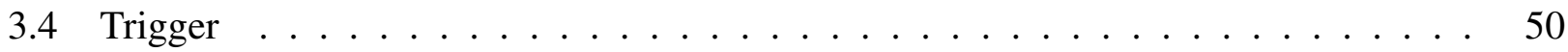

$3.4 .1 \quad$ NIM-based Trigger . . . . . . . . . . . . . . . . . . 51

3.4 .2 FPGA-based Trigger $\ldots \ldots \ldots \ldots \ldots \ldots$. . . . . . . . . . . 51

3.5 Data Acquisition System . . . . . . . . . . . . . . . . . . 54

3.5 .1 Event DAQ . . . . . . . . . . . . . . . . . . 54

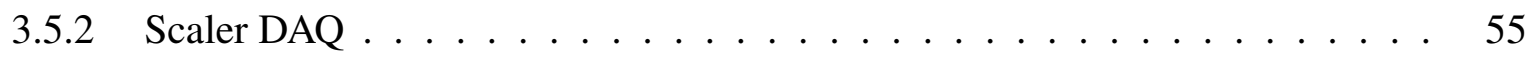

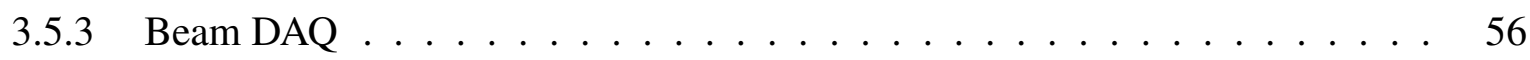


3.6 Monte Carlo Simulation . . . . . . . . . . . . . . . . . . 57

4 Data Analysis $\quad 59$

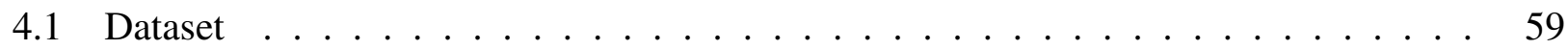

4.2 Dimuon Reconstruction . . . . . . . . . . . . . . . . . . . . 59

4.2.1 Pre-tracking Analysis . . . . . . . . . . . . . . . . . . 60

4.2.1.1 Hit Removal . . . . . . . . . . . . . . . . . 60

4.2.1.2 Occupancy Cut .................... 63

4.2.2 Single Track Reconstruction . . . . . . . . . . . . . . . . . 64

4.2.2.1 Building Tracklets . . . . . . . . . . . . . . . . 64

4.2.2.2 Connecting Tracklets in St. 2 and St. 3 . . . . . . . . . . 68

4.2.2.3 Constructing Global Tracks . . . . . . . . . . . . . . . . . . 69

4.2.3 Reaction Vertex Reconstruction . . . . . . . . . . . . . . . . . 70

4.2.3.1 Single Track Vertex Reconstruction . . . . . . . . . . . . . . 70

4.2.3.2 Dimuon Vertex Reconstruction . . . . . . . . . . . . . . . 74

4.3 Selections and Quality Cuts . . . . . . . . . . . . . . . . . . 74

4.3.1 Spill Level Selection . . . . . . . . . . . . . . . . . . 75

4.3.1.1 Target Position . . . . . . . . . . . . . . . 76

4.3.1.2 Beam Related Quantities . . . . . . . . . . . . . . . 77

4.3.1.3 DAQ Related Quantities . . . . . . . . . . . . . . . . . . 80

4.3.1.4 Magnets Related Quantities . . . . . . . . . . . . . . . . 88

4.3.2 Event Level Selection _. . . . . . . . . . . . . . . . . . . 88

4.3.2.1 Track Selection _ . . . . . . . . . . . . . . . . 88

4.3.2.2 Dimuon Selection . . . . . . . . . . . . . . 96

4.3.2.3 Target-Dump Separation . . . . . . . . . . . . . . . . 103

4.4 Dimuon Yield . . . . . . . . . . . . . . . . . . . . . . . . . . . 109

4.4 Dimuon Raw Yield . . . . . . . . . . . . . . . . . . . 109

4.4 .2 Dimuon Corrected Yield . . . . . . . . . . . . . . . . 110

4.4.2.1 Tracking Efficiency Correction . . . . . . . . . . . . . . 111

4.4.2.2 Empty Target Subtraction . . . . . . . . . . . . . . . . 118

4.4.2.3 Occupancy Dependence of Combinatorial Background . . . . . . 123

4.5 Cross Section Ratio . . . . . . . . . . . . . . . . . . . . . . . . . . 127

4.5.1 Cross Section Ratio Calculation . . . . . . . . . . . . . . . 128

4.5 .2 Error Estimation . . . . . . . . . . . . . . . . . . . 128

$4.5 .3 \quad$ Results . . . . . . . . . . . . . . . . . 132

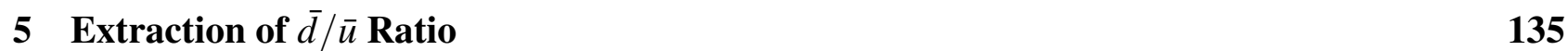

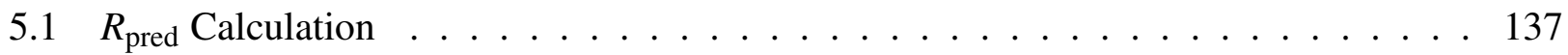

5.2 Update $\bar{d} / \bar{u} \ldots \ldots \ldots \ldots \ldots \ldots \ldots \ldots$. . . . . . . . . . . . . . . . . . . . . . . . . . . . . . . . . . . . . . .

5.3 Error Estimation . . . . . . . . . . . . . . . . . . . . . . . . 138

5.3 .1 Statistical Error . . . . . . . . . . . . . . . . . 138 
5.3 .2 Systematic Error . . . . . . . . . . . . . . . . . . 138

5.4 Results . . . . . . . . . . . . . . . . . . . . . 141

6 Discussion on the Result $\quad 143$

6.1 Comparison with the Previous Results . . . . . . . . . . . . . . . . . . 143

6.2 Comparison with E866 Experiment . . . . . . . . . . . . . . . . . 145

6.3 Origin of Flavor Asymmetry . . . . . . . . . . . . . . . . . . . 145

6.3 .1 Pauli Blocking . . . . . . . . . . . . . . . 146

6.3 .2 Statistical Model . . . . . . . . . . . . . . . . . . . 146

6.3 .3 Pion Cloud Model . . . . . . . . . . . . . . . . . . . . . . . . . . 148

7 Summary and Conclusion $\quad 149$ 


\section{Chapter 1}

\section{Introduction}

Since long time ago, mankind has been interested in the structure of the universe and the matter. The research on the fundamental particles has made a rapid progress only in these 100 years. The reason is that high energy experiments are needed to find such tiny particles.

The electron is the fundamental particle discovered first. The discovery of the electron was made around 1900. Then, Rutherford studied nuclei using the scattering experiment with $\alpha$-particles in 1911. Many hadrons have been found in the following few decades.

The proton had been considered to be a fundamental particle. The deep inelastic scattering (DIS) experiment at SLAC, however, found that the proton is not a fundamental particle but consists of the partons $[3,4]$. The experiment showed that the tiny structure can be investigated with high energy beams. The parton model was proposed by Feynman based on the results of the experiments. The model successfully explains many hadrons with two or three partons (quarks).

However, the gluons have been found at higher energy scale [5]. Quarks are confined into the proton by exchanging the gluons. The gluon in the proton splits into a quark-antiquark pair ( $g \rightarrow d \bar{d}$ or $g \rightarrow u \bar{u}$ ) and then the quark-antiquark pair annihilates back to a gluon. Therefore, the proton consists of not only three quarks but many quarks and antiquarks. These kinds of quarks are called sea quarks.

The gluon splitting is a process of the strong interaction. The strong interaction is described by the Quantum Chromodynamics (QCD). According to the QCD, the gluon splitting is independent of the quark flavor because the coupling constant is the same. This suggests that the amount of $\bar{u}$ in the proton is the same as that of $\bar{d}$ (flavor symmetry). Figure 1.1 shows the proton in different energy scales.



Figure 1.1: Proton structure in different energy scales. The energy increases left to right. 
The NMC experiment at CERN found a violation of the flavor symmetry, namely there are more $\bar{d}$ than $\bar{u}$, by deep inelastic muon scattering [6,7]. The NA51 experiment at CERN measured the flavor asymmetry of the antiquarks using the Drell-Yan process [8]. The NA51 experiment used a $450 \mathrm{GeV}$ proton beam and $\mathrm{H}_{2}$ and $\mathrm{D}_{2}$ targets. NA51 found that the $\bar{d}>\bar{u}$ at Bjorken $x=0.18$ by measuring the cross sections of the $p-p$ and $p-d$ Drell-Yan processes. Here, Bjorken $x$ is the fraction of the momentum of the parton to that of the proton.

The E866 experiment at Fermilab also observed the flavor asymmetry $\bar{d}(x) / \bar{u}(x)$ at $0.015<x<$ 0.35 by the Drell-Yan process $[9,11]$. E866 used the $800 \mathrm{GeV}$ proton beam and $\mathrm{LH}_{2}$ and $\mathrm{LD}_{2}$ targets. E866 found that $\bar{d} / \bar{u}$ deviates from 1.0 upward at $x<0.2$. The $\bar{d} / \bar{u}$ ratio at $x>0.3$, however, seems to be consistent with or less than 1.0 with large statistical errors. More conclusive experimental data at the higher $x$ region is thus needed to understand the structure of the proton.

The SeaQuest/E906 experiment performed at Fermilab is a new experiment to measure the $\bar{d} / \bar{u}$ ratio by the Drell-Yan process. SeaQuest aims to measure $\bar{d} / \bar{u}$ at higher and wider range of $x$ than E866, namely $0.1<x<0.45$. SeaQuest uses a $120 \mathrm{GeV}$ proton beam and $\mathrm{LH}_{2}$ and $\mathrm{LD}_{2}$ targets to measure the muon pairs originating from the Drell-Yan process to obtain the cross section ratio of the $p-p$ and $p-d$ reactions. The advantage of the SeaQuest experiment is to use a lower energy beam and wider acceptance of the spectrometer. The cross section of Drell-Yan is inversely proportional to the beam energy, thus more Drell-Yan dimuons are produced. The wider acceptance spectrometer allows to detect such higher and wider range of Bjorken $x$. The SeaQuest provides the precise $\bar{d} / \bar{u}$ result at high $x$ region.

The detector construction, detector performance evaluation, data taking and data analysis for the flavor asymmetry of the antiquarks $\bar{d} / \bar{u}$ in the proton were my contributions to SeaQuest. An analysis method was developed based on the chamber occupancy of the drift chambers. It is a key element in the analysis which handles reactions with high intensity proton beam. This thesis is organized in the following way. In Chapter 2, the physics background and the history of the study are explained. In Chapter 3, the experimental setup including the spectrometer is shown. Chapter 4 is the main part. It explains the data analysis to obtain the cross section ratio of $\sigma^{p p} / 2 \sigma^{p d}$. In Chapter 5 , the $\bar{d} / \bar{u}$ extraction method using the Chapter 4 results is explained. The results are discussed in Chapter 6. Chapter 7 is a summary. 


\section{Chapter 2}

\section{Nucleon Structure}

The SeaQuest experiment aims to measure the structure of the nucleon. Many experiments have investigated the structure of the nucleon so far. The deep inelastic scattering and the Drell-Yan process are mainly used for the investigation.

The theoretical background and the history of the investigation on the nucleon structure is explained in this chapter.

\subsection{Deep Inelastic Scattering}

In the lepton-proton scattering, the excited states of the nucleon appear at $W<4 \mathrm{GeV}$. Here, $W$ is the invariant mass of the hadrons in the resonance states in this region. However, the resonance states don't appear at $W \geq 4 \mathrm{GeV}$. At $W \geq 4 \mathrm{GeV}$, the lepton beam breaks the proton and interact with components of the proton at this $W$ range. The lepton-proton reaction at this $W$ range is called deep inelastic scattering (DIS). As mentioned above, this reaction can access the components of the proton. Therefore, the DIS is one of the important tools for investigating the nucleon structure.

Figure 2.1 is a drawing of the deep inelastic scattering. The lepton exchanges the virtual boson

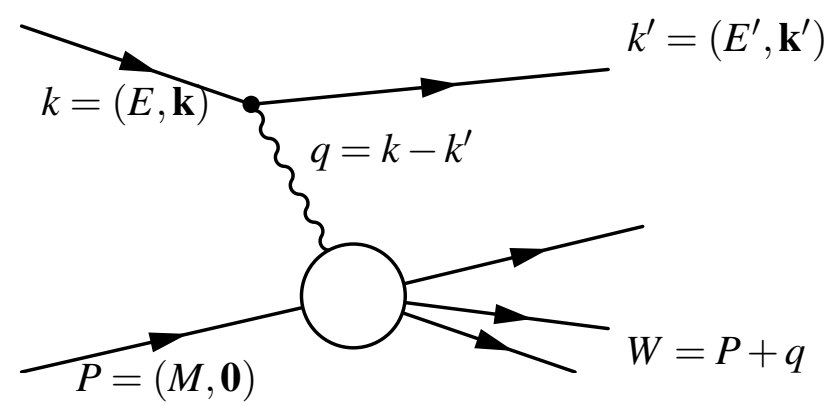

Figure 2.1: Drawing of deep inelastic scattering. $k$ and $k^{\prime}$ are 4-momenta of initial and final state leptons, respectively. $q$ is the 4 -momentum of virtual photon. $P$ is the 4-momentum of the initial state proton. $W$ is the invariant mass of hadron in the final state.

with a constituent of the proton. The type of the boson exchanged in the reaction depends on the energy scale. Here, only the virtual photon is taken into account because the electromagnetic interaction is dominant in the energy scale of the experiments related to this thesis. 
The cross section of the DIS is described as

$$
\frac{d^{2} \sigma}{d \Omega d E^{\prime}}=\frac{\alpha^{2}}{4 E^{\prime 2} \sin ^{4}(\theta / 2)}\left[\frac{1}{v} F_{2}\left(x, Q^{2}\right) \cos ^{2} \frac{\theta}{2}+\frac{1}{M} F_{1}\left(x, Q^{2}\right) \sin ^{2} \frac{\theta}{2}\right],
$$

where $F_{1}$ and $F_{2}$ are the structure functions. The other kinematic variables used in Eq. (2.1) are listed in Tab. 2.1.

Table 2.1: Kinematic variables in DIS.

\begin{tabular}{ll}
\hline$k, k^{\prime}$ & 4-momenta of the initial and final state leptons \\
$q$ & 4-momentum of the virtual photon \\
$P$ & 4-momentum of the initial state proton \\
$W$ & invariant mass of hadron in the final state \\
$\Omega$ & solid angle in the scattered lepton detection \\
$\theta$ & scattering angle of the lepton \\
$v=E-E^{\prime}$ & energy of the virtual photon \\
$x=\frac{Q^{2}}{2 P \cdot q}$ & Bjorken scaling variable \\
$Q^{2}=-q^{2}$ & momentum transfer squared \\
\hline
\end{tabular}

The structure functions $F_{1}$ and $F_{2}$ are functions of $x$ and $Q^{2}$. The cross section of lepton-proton scattering decreases rapidly as $Q^{2}$ increases, but the structure functions do not depend on $Q^{2}$ in the first approximation [12]. Therefore, they can be written as the functions of only $x: F_{1}(x)$ and $F_{2}(x)$ (Bjorken scaling). Here, $x$ is called Bjorken scaling variable defined as

$$
x=\frac{Q^{2}}{2 P \cdot q}=\frac{Q^{2}}{2 M v} .
$$

The Bjorken scaling indicates that the proton has point-like particles. These particles are called parton, and this nucleon structure model is called parton model [15]. The partons were found to be identical with quarks and gluons, later.

In the parton model, the interaction between partons can be ignored in DIS: a parton can be treated as a free particle. This is called inpulse approximation. It is because the interaction time of virtual photon is much shorter than that between partons. Therefore, the cross section of lepton-proton reaction can be written as the sum of the cross sections of lepton-partons:

$$
\sigma^{l p}=\sum_{\text {parton }} \sigma^{l \text { parton }}
$$

The structure functions can also be written as

$$
\begin{aligned}
& F_{1}=\frac{1}{2} \sum_{i} z_{i}^{2}\left(q_{i}(x)+\bar{q}_{i}(x)\right), \\
& F_{2}=x \sum_{i} z_{i}^{2}\left(q_{i}(x)+\bar{q}_{i}(x)\right),
\end{aligned}
$$


where $z_{i}$ is the charge of the $q_{i}$, and $q_{i}(x)$ is the parton distribution function (PDF). The PDF is the probability that the parton exists between $x$ and $x+d x$.

The meaning of the Bjorken variable is provided with the parton model. Assuming the case that the longitudinal momentum of the proton is much larger than transverse momentum, the momentum of parton in the proton should also be longitudinal. In this case, the parton is considered to have a partial momentum of the proton: $p_{\text {parton }}=\xi p_{\text {proton }}$. Under the inpulse approximation, the virtual photon with a four-component momentum $q$ interacts with a free parton. The invariant mass of the virtual photon-parton interaction is described as

$$
\left(\xi p_{\text {proton }}+q\right)^{2}=m^{2}
$$

where $m$ is the parton mass in the final state. The parton mass is ignored since it is small compared to momenta:

$$
\left(\xi p_{\text {proton }}+q\right)^{2}=0
$$

From Eq. (2.6),

$$
\xi=\frac{Q^{2}}{2 P \cdot q}=x
$$

is extracted. The Bjorken scaling variable indicates the fraction of the momentum of the parton to that of the proton at DIS energy scale.

\subsection{Quantum Chromodynamics (QCD)}

The parton model succeeded in explaining the simple structure of the nucleon. However, for example, there is a problem if the quark model is applied to $\Delta^{++}(1232)$ : it consists of three $u$-quarks (uuu). The spin of each $u$-quark should be the same direction since the spin of $\Delta^{++}(1232)$ is $3 / 2$. This is inconsistent with the Pauli exclusion principle. This problem was solved by introducing a new intrinsic charge: color. There three types of colors: Red, Green, and Blue. The problem is solved by adding the colors to $\Delta^{++}: u_{R} u_{G} u_{B}$, for example.

The color is a charge for the strong interaction. The theory for the color charge and the strong interaction is called Quantum Chromodynamics (QCD). The Lagrangian of the QCD is

$$
\mathscr{L}_{\mathrm{QCD}}=\bar{\psi}\left(i \gamma^{\mu} \mathscr{D}_{\mu}-m\right) \psi-\frac{1}{4} F_{\mu \nu}^{a}-\frac{1}{2 \alpha}\left(\partial^{\mu} A_{\mu}^{a}\right)^{2}-\bar{c}^{a} \partial^{\mu} \mathscr{D}_{\mu}^{a b} c^{b}
$$

The first term corresponds to the fermions and their interactions.

$$
\mathscr{D}_{\mu} \psi(x)=\left[\partial_{\mu}-i g T^{a} A_{\mu}^{a}(x)\right] \psi(x)
$$

where $A_{\mu}^{a}$ is a gluon, and $g$ is a coupling constant. The second term is a kinematic term. This term includes $\left[A_{\mu}, A_{v}(x)\right]$ which is a unique noncommutative term in the QCD. This term causes the asymptotic freedom [13] and the quark confinement. The third term is called gauge fixing term. The fourth term arises from Faddeev-Popov ghost [14]. 
The effective coupling constant of the QCD is expressed as

$$
\alpha_{s}\left(Q^{2}\right)=\frac{4 \pi}{\beta_{0} \ln \left(Q^{2} / \Lambda^{2}\right)},
$$

where $\beta_{0}$ is the leading-order of the $\beta$ function expanded with coupling constant $g$. Eq. (2.10) shows that the coupling constant is independent of the quark flavor. Therefore, the processes caused by the QCD such as gluon splitting are expected to be independent of quark flavor. Eq. (2.10) is inversely proportional to $\ln Q^{2}$, so coupling constant becomes small as $Q^{2}$ becomes large. It means that the particle is free at high energy (very short distance). This phenomenon is unique in the QCD, and is called asymptotic freedom.

The PDFs themselves cannot be calculated by QCD. The $x$ dependence of the PDFs can be calculated with the effective models, but the functions in the model is highly dependent of the parameters. Therefore, it is important to obtain the PDFs experimentally. Figure 2.2 is an example of the PDF calculation [18].

MSTW 2008 NLO PDFs (68\% C.L.)
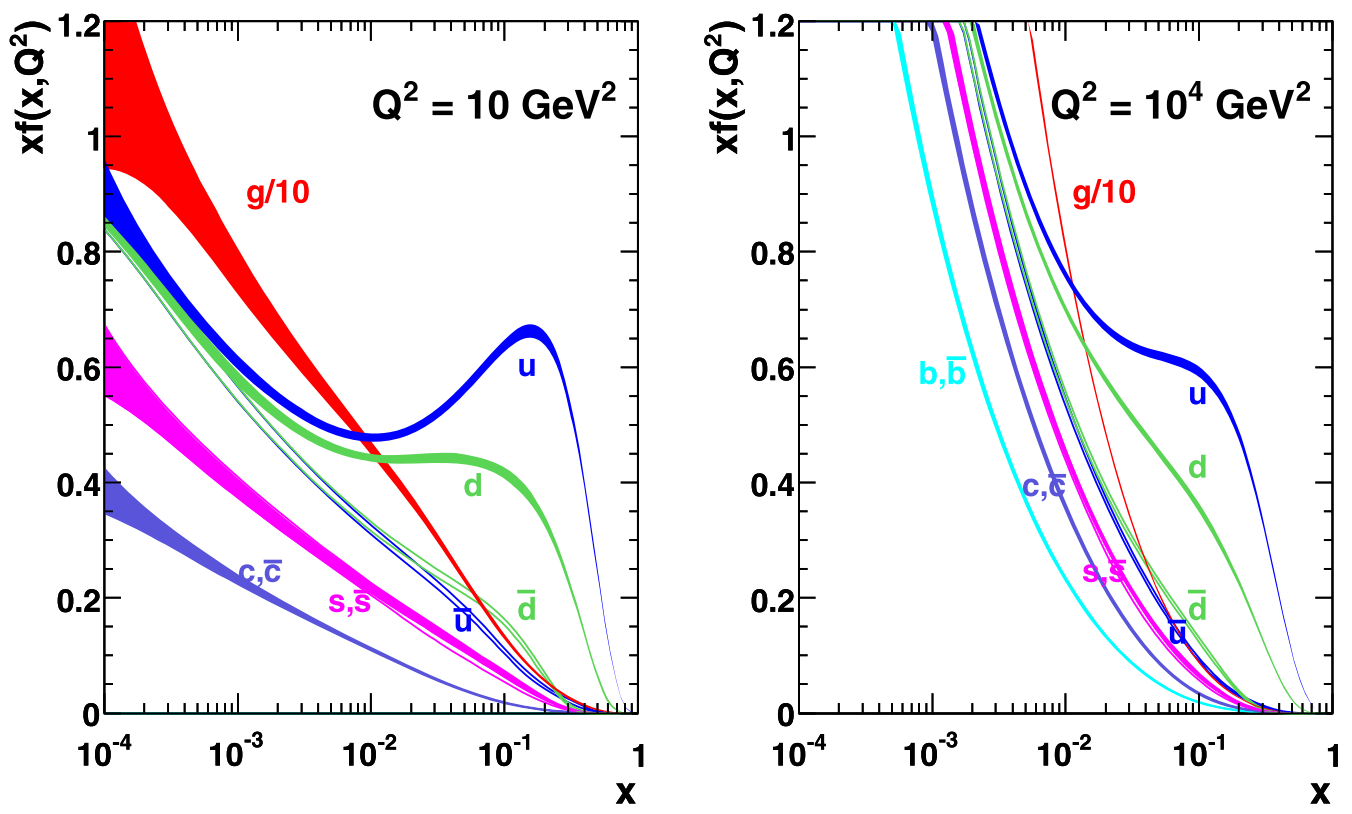

Figure 2.2: NLO PDF calculation at $Q^{2}=10 \mathrm{GeV}^{2}$ and $Q^{2}=10^{4} \mathrm{GeV}^{2}$.

\subsection{Drell-Yan Process}

The SeaQuest experiment uses the Drell-Yan process [17]. An antiquark in a hadron and a quark in another hadron annihilate and create a virtual photon, which then decays into a lepton pair in the Drell-Yan process. SeaQuest uses the proton as hadrons and measures the muon pairs as lepton pairs $\left(q \bar{q} \rightarrow \gamma^{*} \rightarrow \mu^{+} \mu^{-}\right)$. The diagram of the Drell-Yan process is shown in Fig. 2.3. 


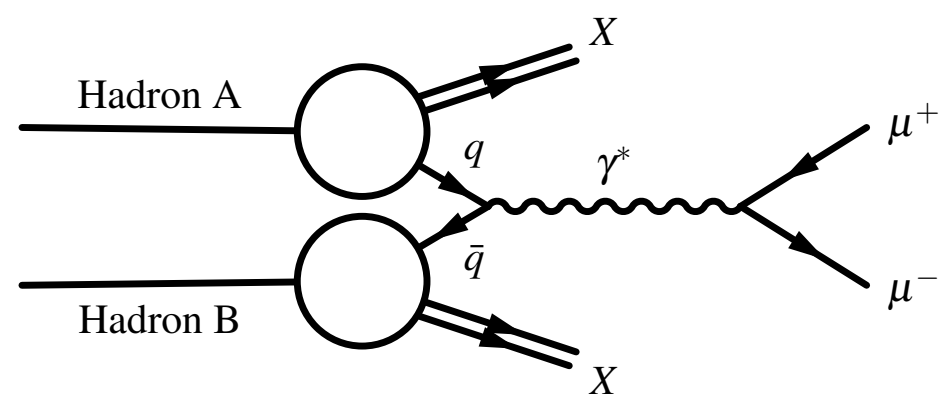

Figure 2.3: Drawing of Drell-Yan process.

The cross section of the Drell-Yan process is

$$
\frac{d^{2} \sigma}{d x_{1} d x_{2}}=\frac{4 \pi \alpha}{9 M^{2}} \sum_{i} e_{i}^{2}\left[q_{i}\left(x_{1}\right) \bar{q}_{i}\left(x_{2}\right)+\bar{q}_{i}\left(x_{1}\right) q_{i}\left(x_{2}\right)\right],
$$

where $x_{1}$ and $x_{2}$ are Bjorken $x$ of the partons in the beam and that in the target, respectively, and $M$ is the invariant mass of the lepton pair.

The advantages of the Drell-Yan process are

- the antiquark PDF is always involved in the reaction, and

- the kinematics is simple and can be determined experimentally.

The antiquark PDF is directly related with the Drell-Yan cross section as shown in Eq. (2.11). The typical kinematic acceptance of the SeaQuest experiment is $x_{1} \gg x_{2}$ as shown in Fig. 2.4. The PDFs of antiquarks are small and quark-PDFs are dominant at high $x$ region as shown in Fig. 2.2. Therefore, the second term of Eq. (2.11) can be ignored at the SeaQuest kinematic acceptance:

$$
\frac{d^{2} \sigma}{d x_{1} d x_{2}} \approx \frac{4 \pi \alpha}{9 M^{2}} \sum_{i} e_{i}^{2}\left[q_{i}\left(x_{1}\right) \bar{q}_{i}\left(x_{2}\right)\right] .
$$

Then, the antiquark of the target proton can be directly accessed.

The kinematic variables are calculated with the variables at the center of mass frame: the center of mass energy of the interacting hadrons $\sqrt{s}$, the mass of the lepton pair $M$, longitudinal momentum $p_{l}$, and the energy of the virtual photon $E$.

$$
\begin{aligned}
\tau & =M^{2} / s=x_{1} x_{2}, \\
y & =\frac{1}{2} \ln \left(\frac{E+p_{l}}{E-p_{l}}\right), \\
x_{1} & =\sqrt{\tau} e^{y}, \\
x_{2} & =\sqrt{\tau} e^{-y}, \\
x_{F} & =x_{1}-x_{2} .
\end{aligned}
$$




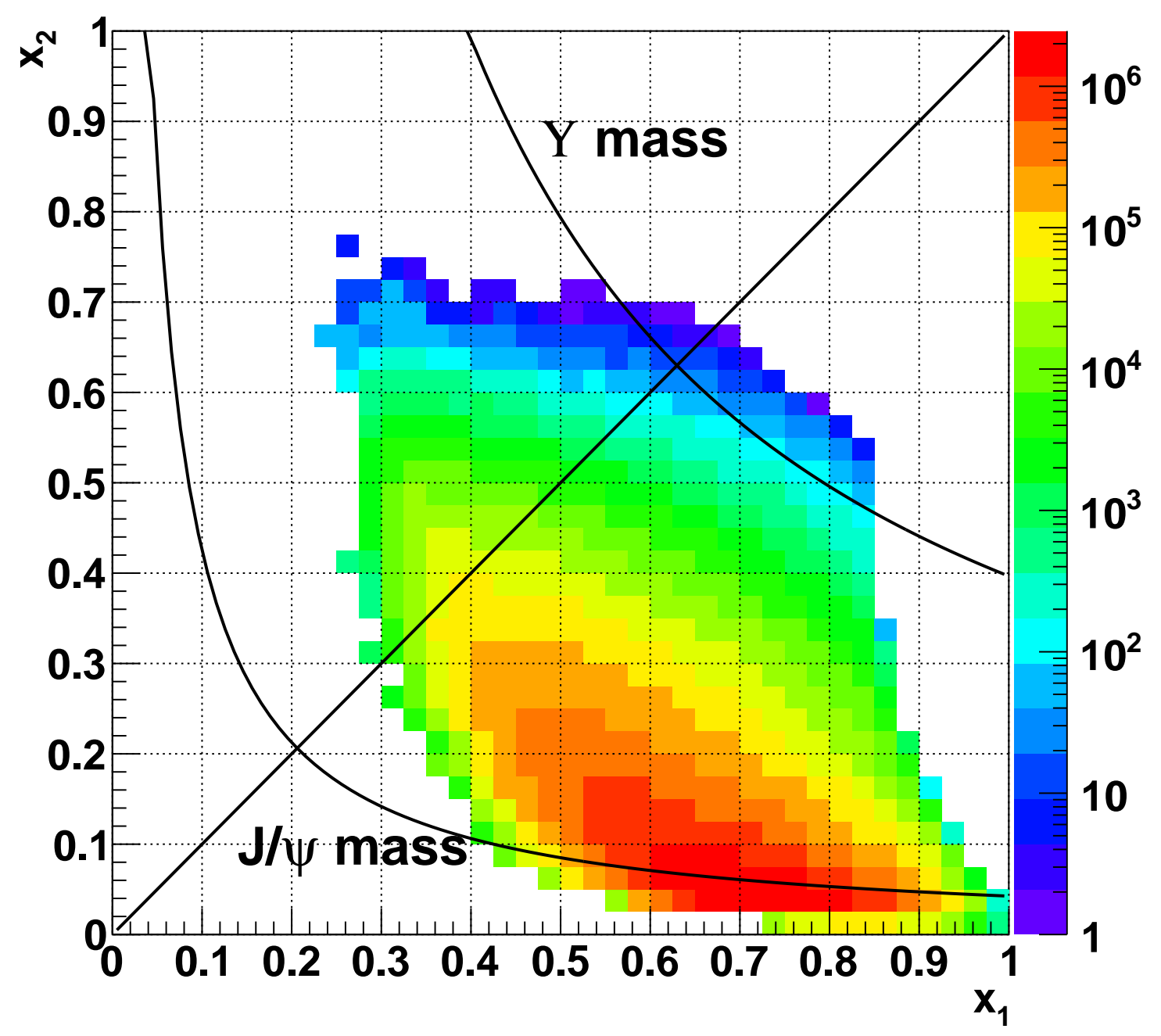

Figure 2.4: Acceptance of the SeaQuest spectrometer. The Geant4-based Monte-Carlo simulation data (Section 3.6) are used in the figure. The curves corresponding to $J / \psi$ mass and $\Upsilon$ mass are also shown. 


\subsection{Motivation for the SeaQuest Experiment}

One of the challenges of the QCD is to understand the flavor asymmetry of the antiquarks. The PDFs of $\bar{u}$ and $\bar{d}$ have been considered to be the same because the process of the gluon splitting is independent of the quark flavor. This section shows the history of the experimental investigations of the antiquark PDFs and the motivation for the SeaQuest experiment.

\subsubsection{NMC Experiment}

The first attempt to investigate the flavor symmetry was to measure the Gottfried sum rule [19]. Here, the flavor symmetry, if valid, leads to $\int_{0}^{1} d x \bar{u}(x)=\int_{0}^{1} d x \bar{d}(x)$. The Gottfried sum rule is a rule for the structure function of the proton $F_{2}^{p}$ and that of the neutron $F_{2}^{n}$. Using the parton distribution functions of quarks and antiquarks, the leading-order structure function of the proton is

$$
F_{2}^{p}(x)=x\left[\frac{4}{9}(u(x)+\bar{u}(x))+\frac{1}{9}\left(d(x)+\bar{d}(x)+\sum_{i} z_{i}^{2}\left(q_{i}(x)+\bar{q}_{i}(x)\right)\right)\right],
$$

where $i$ is for strange quark and heavy quarks. Under the assumption of the isospin symmetry, namely $u_{p}(x)=d_{n}(x), \bar{u}_{p}(x)=\bar{d}_{n}(x), d_{p}(x)=u_{n}(x)$ and $\bar{d}_{p}(x)=\bar{u}_{n}(x)$, the structure function of the neutron is

$$
F_{2}^{n}(x)=x\left[\frac{4}{9}(d(x)+\bar{d}(x))+\frac{1}{9}\left(u(x)+\bar{u}(x)+\sum_{i} z_{i}^{2}\left(q_{i}(x)+\bar{q}_{i}(x)\right)\right)\right] .
$$

As a result, the integrated difference between $F_{2}^{p}$ and $F_{2}^{n}$ is

$$
\begin{aligned}
S_{G} & =\int_{0}^{1} d x\left(F_{2}^{p}(x)-F_{2}^{n}(x)\right) / x \\
& =\int_{0}^{1}\left[\frac{1}{3}(u(x)+\bar{u}(x))-\frac{1}{3}(d(x)+\bar{d}(x))\right] \\
& =\int_{0}^{1}\left[\frac{1}{3}(u(x)-\bar{u}(x))-\frac{1}{3}(d(x)-\bar{d}(x))+\frac{2}{3}(\bar{u}(x)-\bar{d}(x))\right] .
\end{aligned}
$$

Since the constituent quarks of the proton are two $u$-quarks and one $d$-quark, the following relations are established:

$$
\begin{aligned}
& \int_{0}^{1} d x(u(x)-\bar{u}(x))=2, \\
& \int_{0}^{1} d x(d(x)-\bar{d}(x))=1 .
\end{aligned}
$$

From Eq. (2.22), Eq. (2.23) and Eq. (2.24), $S_{G}$ is expressed as

$$
S_{G}=\frac{1}{3}+\frac{2}{3} \int_{0}^{1} d x(\bar{u}(x)-\bar{d}(x)) .
$$

If the flavor symmetry is assumed, the second term of Eq. (2.25) vanishes:

$$
S_{G}=\int_{0}^{1} d x\left(F_{2}^{p}(x)-F_{2}^{n}(x)\right) / x=\frac{1}{3} .
$$




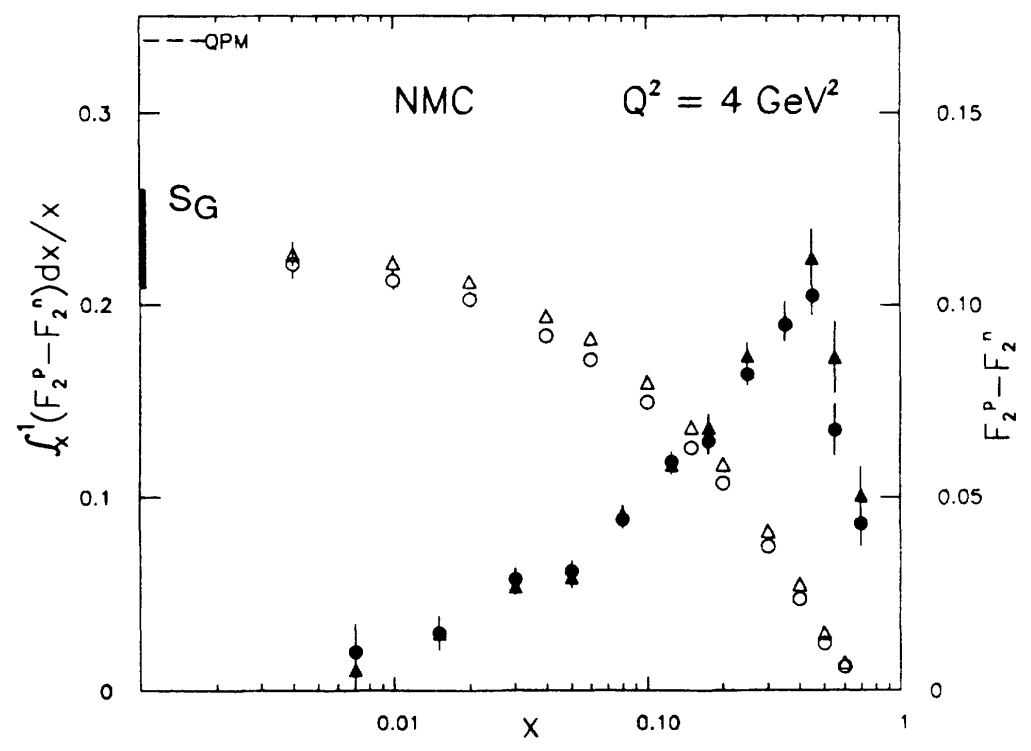

Figure 2.5: NMC results of $F_{2}^{p}(x)-F_{2}^{n}(x)$ (black points) and $\int_{x}^{1}\left(F_{2}^{p}(x)-F_{2}^{n}(x)\right) d x / x$ (white points) [7]. Circles are the reevaluation results from Ref. [7], and triangles are from Ref. [6]. The black line on the left vertical axis is the extrapolated result $\left(S_{G}\right)$. The prediction based on the simple quark-parton model (QPM) is also shown.

Eq. (2.26) is called the Gottfried sum rule.

The early experiments measured the Gottfried sum, but they were not able to conclude whether the Gottfried sum rule is valid or not due to the large errors [20-23]. The first successful measurement for investigating the Gottfried sum rule was performed by the NMC experiment at CERN $[6,7,24]$. The NMC experiment is a deep inelastic muon scattering experiment. It uses 90 and $280 \mathrm{GeV} / \mathrm{c}$ muon beams and hydrogen and deuterium targets. The kinematic range of the experiment is $x=0.004-$ 0.8 and $Q^{2}=1-190 \mathrm{GeV}^{2}$. The measured quantity is $F_{2}^{n} / F_{2}^{p}=F_{2}^{d} / F_{2}^{p}-1$. Using the result of measurement, the difference of the structure functions is derived:

$$
F_{2}^{p}-F_{2}^{n}=2 F_{2}^{d} \frac{1-F_{2}^{n} / F_{2}^{p}}{1+F_{2}^{n} / F_{2}^{p}} .
$$

Figure 2.5 shows the NMC result of $F_{2}^{p}(x)-F_{2}^{n}(x)$ (black points) and $\int_{x}^{1}\left(F_{2}^{p}(x)-F_{2}^{n}(x)\right) d x / x$ (white points) at $Q^{2}=4 \mathrm{GeV}^{2}$. The partial Gottfried sum for $0.004<x<0.8$ at $Q^{2}=4 \mathrm{GeV}^{2}$ is

$$
S_{G}(0.004,0.8)=0.221 \pm 0.008(\text { stat }) \pm 0.019(\text { syst })
$$

To obtain the complete Gottfried sum, the contributions at $x>0.8$ and $x<0.004$ to Gottfried sum were estimated by extrapolating $F_{2}^{p}(x)-F_{2}^{n}(x)$ to $x=0$ and $x=1$. The NMC experiment extracted the Gottfried sum as

$$
S_{G}=0.235 \pm 0.026
$$

at $Q^{2}=4 \mathrm{GeV}^{2}$. The result is significantly below the simple quark-parton model expectation of $1 / 3$. It suggests $\int_{0}^{1} d x \bar{d}(x)>\int_{0}^{1} d x \bar{u}(x)$. 


\subsubsection{NA51 Experiment}

There are two scenarios to explain the result of NMC experiment. The first scenario is $\int_{0}^{1} d x \bar{d}(x)>$ $\int_{0}^{1} d x \bar{u}(x)$, which means $\bar{d}(x) \neq \bar{u}(x)$. The second one is that the isospin symmetry is broken, namely $\bar{u}_{p}(x) \neq \bar{d}_{n}(x)$. Here, the isospin symmetry was considered to be preserved, and the first one was further investigated.

The Drell-Yan process is suitable for the research of the antiquarks. The NA51 experiment performed at CERN [8] measured the Drell-Yan cross sections of $p-p$ and $p-n$ collisions. NA51 used a $450 \mathrm{GeV} / c$ proton beam and $\mathrm{LH}_{2}$ and $\mathrm{LD}_{2}$ are used as targets. NA51 measured the cross section asymmetry:

$$
\begin{aligned}
A_{D Y} & =\frac{\sigma^{p p}-\sigma^{p n}}{\sigma^{p p}+\sigma^{p n}} \\
& =2 \frac{\sigma^{p p}}{\sigma^{p d}}-1,
\end{aligned}
$$

where $\sigma^{p p}$ and $\sigma^{p n}$ are the cross sections for the dimuons produced in $p-p$ and $p-n$ reactions, respectively. Here, $\sigma^{p d}=\sigma^{p p}+\sigma^{p n}$, where $\sigma^{p d}$ is the cross section of $p-d$ reaction, is assumed. Eq. (2.30) can also be written as

$$
A_{D Y}=\frac{\left(4 u_{v}-d_{v}\right)(\bar{u}-\bar{u})+\left(u_{v}-d_{v}\right)(4 \bar{u}-\bar{d})}{\left(4 u_{v}+d_{v}\right)(\bar{u}+\bar{u})+\left(u_{v}+d_{v}\right)(4 \bar{u}+\bar{d})}
$$

where $u_{v}, d_{v}, \bar{u}$ and $\bar{d}$ are the parton distribution functions of valence $u$-quark, valence $d$-quark, $\bar{u}$ quark and $\bar{d}$-quark, respectively. This equation is simplified with the ratios of up quark to down quark:

$$
A_{D Y}=\frac{\left(4 \lambda_{v}-1\right)\left(\lambda_{s}-1\right)+\left(\lambda_{v}-1\right)\left(4 \lambda_{s}-1\right)}{\left(4 \lambda_{v}+1\right)\left(\lambda_{s}+1\right)+\left(\lambda_{v}+1\right)\left(4 \lambda_{s}+1\right)},
$$

where $\lambda_{v}$ is the ratio of the valence $u$-quark distribution to the valence $d$-quark distribution, $u_{v}(x) / d_{v}(x)$, and $\lambda_{s}$ is the ratio of the $\bar{u}$-quark distribution to the $\bar{d}$-quark distribution in the proton, $\bar{u}(x) / \bar{d}(x)$. The NA51 obtained the asymmetry as

$$
A_{D Y}=-0.09 \pm 0.02(\text { stat }) \pm 0.025 \text { (syst) }
$$

The ratio $\lambda_{s}=\bar{u} / \bar{d}$ is obtained using Eq. (2.33) as

$$
\bar{d} / \bar{u}=1.96 \pm 0.15 \text { (stat) } \pm 0.19 \text { (syst) }
$$

at $x=0.18$. This result suggests the flavor symmetry violation of the light quark sea in the proton. The $\bar{d}$-quark exceeds the $\bar{u}$-quark in the proton. The result agrees with the NMC result.

\subsubsection{E866/NuSea Experiment}

The NA51 experiment suggested the violation of the light quark flavor symmetry. The result was however provided at only one $x$ value. A more detailed answer on flavor asymmetry was provided by 
the E866/NuSea experiment performed at Fermilab [9, 10]. E866 used a $800 \mathrm{GeV} / c$ proton beam and liquid hydrogen and liquid deuterium targets. Approximately 360,000 Drell-Yan muon pairs were used for the analysis. The goal of the experiment was to obtain the flavor asymmetry of antiquarks $\bar{d} / \bar{u}$ for $0.015<x<0.35$. The E866 experiment measured the Drell-Yan cross sections of $p-p$ and $p-n$ reactions: $\sigma^{p p}$ and $\sigma^{p n}$. In the E866 experiment, the kinematic acceptance of $x$ in beam $\left(x_{1}\right)$ is much larger than $x$ in target $\left(x_{2}\right)$, namely $x_{1} \gg x_{2}$. Thus, the cross sections of $p-p$ and $p-n$ reactions for $x_{1} \gg x_{2}$ can be expressed as

$$
\begin{aligned}
\sigma^{p p} & \propto \frac{4}{9} u\left(x_{1}\right) \bar{u}\left(x_{2}\right)+\frac{1}{9} d\left(x_{1}\right) \bar{d}\left(x_{2}\right), \\
\sigma^{p n} & \propto \frac{4}{9} u\left(x_{1}\right) \bar{d}\left(x_{2}\right)+\frac{1}{9} d\left(x_{1}\right) \bar{u}\left(x_{2}\right) .
\end{aligned}
$$

The Drell-Yan cross section ratio of $p-d$ reaction to $p-p$ reaction is

$$
\begin{aligned}
\frac{\sigma^{p d}}{2 \sigma^{p p}} & =\frac{1}{2} \frac{\sigma^{p p}+\sigma^{p n}}{\sigma^{p p}} \\
& \approx \frac{1}{2} \frac{\frac{4}{9} u\left(x_{1}\right) \bar{u}\left(x_{2}\right)+\frac{1}{9} d\left(x_{1}\right) \bar{d}\left(x_{2}\right)+\frac{4}{9} u\left(x_{1}\right) \bar{d}\left(x_{2}\right)+\frac{1}{9} d\left(x_{1}\right) \bar{u}\left(x_{2}\right)}{\frac{4}{9} u\left(x_{1}\right) \bar{u}\left(x_{2}\right)+\frac{1}{9} d\left(x_{1}\right) \bar{d}\left(x_{2}\right)} \\
& =\frac{1}{2} \frac{1+\frac{1}{4} \frac{d\left(x_{1}\right)}{u\left(x_{1}\right)}}{1+\frac{1}{4} \frac{d\left(x_{1}\right)}{u\left(x_{1}\right)} \frac{\bar{d}\left(x_{2}\right)}{\bar{u}\left(x_{2}\right)}}\left[1+\frac{\bar{d}\left(x_{2}\right)}{\bar{u}\left(x_{2}\right)}\right]
\end{aligned}
$$

The equation can be more simplified with $d(x) \ll 4 u(x)$,

$$
\frac{\sigma^{p d}}{2 \sigma^{p p}} \approx \frac{1}{2}\left[\frac{\bar{d}}{\bar{u}}+1\right] .
$$

The E866 experiment extracted $\bar{d} / \bar{u}$ for $0.015<x<0.35$ by measuring the cross section ratio.

Figure 2.6 shows the results of E866 experiment together with the NA51 result. The E866 experiment showed many data points of $\bar{d} / \bar{u}$ and confirmed that the flavor symmetry is broken at $0.015<x<0.35$. The $\bar{d} / \bar{u}$ is 1.7 at maximum at $x \sim 0.2$, which is consistent with the tendency of the NMC experiment. The behavior is investigated theoretically and several models have been proposed [25,26]. On the other hand, $\bar{d} / \bar{u}$ at $x \sim 0.3$ is close to 1.0 or less with large statistical uncertainty. This behavior is not reproduced by any theoretical models. The precise $\bar{d} / \bar{u}$ measurement for higher $x$ region is needed.

The CTEQ group has calculated the parton distribution functions based on the results of experiments. The CTEQ4M [27] PDF in 1997 was calculated without the E866 results. The next version, CTEQ5M [28] PDF in 2000, was calculated with E866 results. The effect of the E866 results is shown in Fig. 2.7. The effect is significant at large $x$ region. 


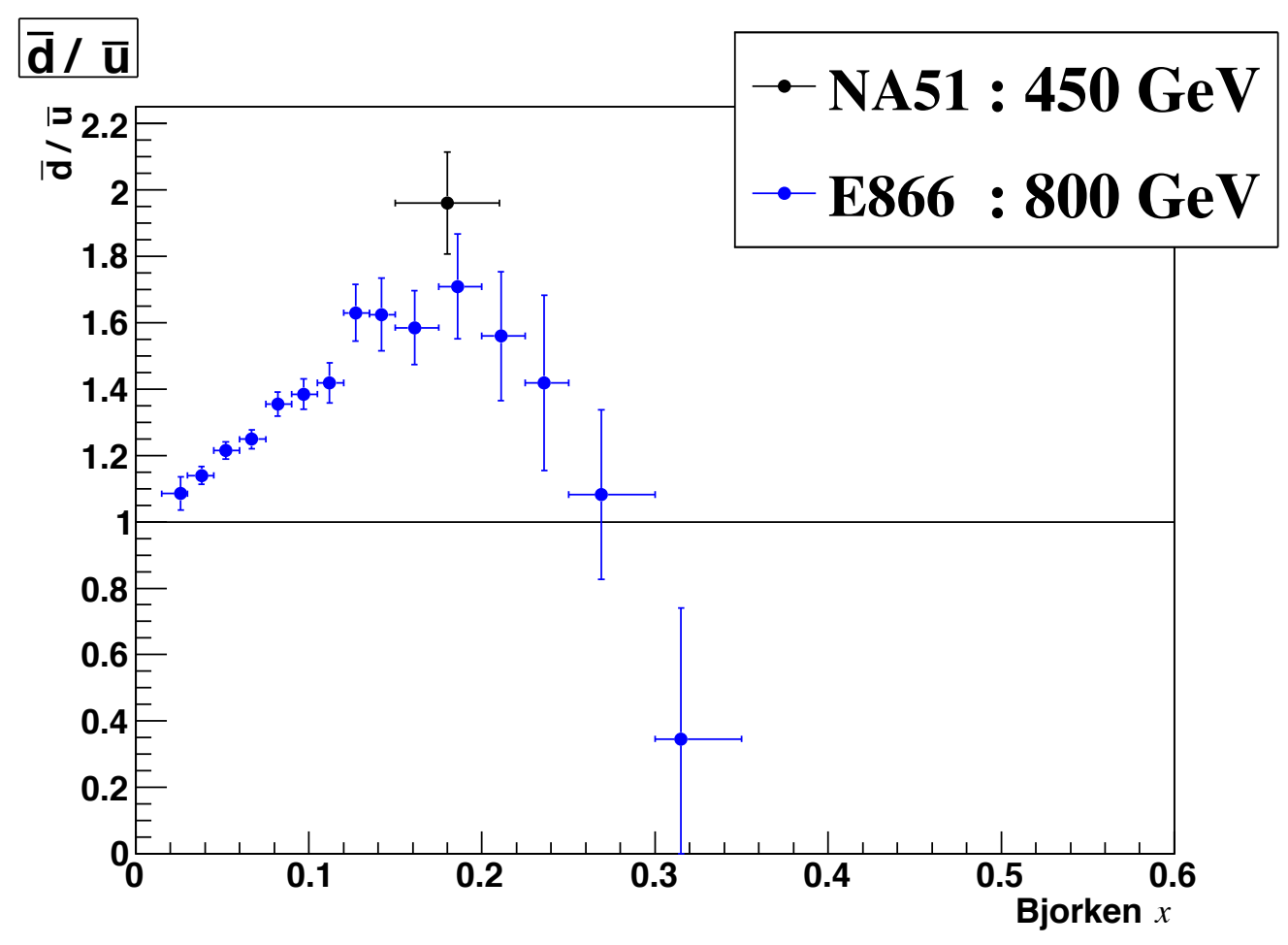

Figure 2.6: Results of E866 experiment and NA51 experiment. The blue points are the results of the E866 experiment, and a black point is the result of the NA51 experiment.



Figure 2.7: Comparison of the $\bar{d} / \bar{u}$ distribution in CTEQ4M and CTEQ5M [28]. The major change is caused by the E866 data. 


\subsubsection{Motivation of SeaQuest Experiment}

The E866 experiment succeeded in obtaining the precise data in the small $x$ region, but the $\bar{d} / \bar{u}$ at $x>0.24$ is not conclusive due to the large uncertainty. A new experiment, SeaQuest (E906), aims to determine the $\bar{d} / \bar{u}$ at higher and wider $x$ region $(0.1<x<0.45)$ precisely [29]. SeaQuest will provide new data points and contribute to understanding the proton structure.

The SeaQuest spectrometer is designed based on the E866 spectrometer. SeaQuest focuses on the high $x$ Drell-Yan dimuons, thus the acceptance of the spectrometer is wider than the E866 spectrometer. The energy of the proton beam is different between the SeaQuest and the E866 experiments: $120 \mathrm{GeV}(\sqrt{s} \sim 15 \mathrm{GeV})$ and $800 \mathrm{GeV}(\sqrt{s} \sim 40 \mathrm{GeV})$. The low beam energy provides two advantages: high statistics and low background rate.

The Drell-Yan cross section is inversely proportional to $s$. Therefore, the Drell-Yan cross section at the SeaQuest is larger than that at the E866 experiment.

The data acquisition is suppressed if the background rate is high. The main background for the Drell-Yan experiments is muons from $J / \psi$ decay. The $J / \psi$ production is roughly proportional to the beam energy. Thus, the $J / \psi$ event rate is suppressed in the SeaQuest experiment. 


\section{Chapter 3}

\section{SeaQuest Experiment}

The SeaQuest experiment is a Drell-Yan experiment at Fermi National Accelerator Laboratory (Fermilab). The collaboration consists of Japanese, American and Taiwanese institutes. The SeaQuest experiment uses the $120 \mathrm{GeV}$ proton beam provided by the Fermilab Main Injector and uses fixed targets. The targets are liquid hydrogen and deuteron, and solid nuclear targets. For the measurement of the flavor asymmetry of antiquarks in the proton, SeaQuest measures the momenta of the muons produced in the Drell-Yan process.

The timeline of the SeaQuest experiment is shown in Tab. 3.1. The spectrometer construction started in 2009. The commissioning run was performed in 2012. After the commissioning run, a one-and-half-year detector upgrade was done. The main upgrade in this period was to install one of the new drift chambers. The first physics data acquisition started in November 2013. Three data acquisition periods have been completed. After the accelerator shutdown, the fourth physics data acquisition started and is in progress.

Figure 3.1 shows the integrated number of protons on target. The recorded protons on target so far is $1.1 \times 10^{18}$ protons. After the fourth data acquisition, the total integrated protons on target will be $1.5 \times 10^{18}$ protons. The number of the protons written in the proposal is $3.4 \times 10^{18}$. Physics data taking after the present run will be determined later.

This chapter explains the devices to detect the muon pairs originating from the Drell-Yan process. 
Table 3.1: Timeline of SeaQuest experiment.

\begin{tabular}{ccl}
\hline Year & Month & Description \\
\hline 2009 & 04 & Building detectors \\
2011 & 08 & \\
2012 & $03-04$ & Commissioning Run (Run I) \\
& $05-$ & Accelerator Shutdown \& Detector upgrade (installed St. 3- drift chamber) \\
2013 & $11-$ & First Physics Data Acquisition (Run II) \\
2014 & $09-10$ & Accelerator Shutdown \\
& $11-$ & Second Physics Data Acquisition (Run III) \\
2015 & $07-09$ & Accelerator Shutdown \\
& $10-$ & Third Physics Data Acquisition (Run IV) \\
2016 & $08-11$ & Accelerator Shutdown \\
2016 & $11-$ & Fourth Phyics Data Acquisition (Run V) \\
\hline
\end{tabular}

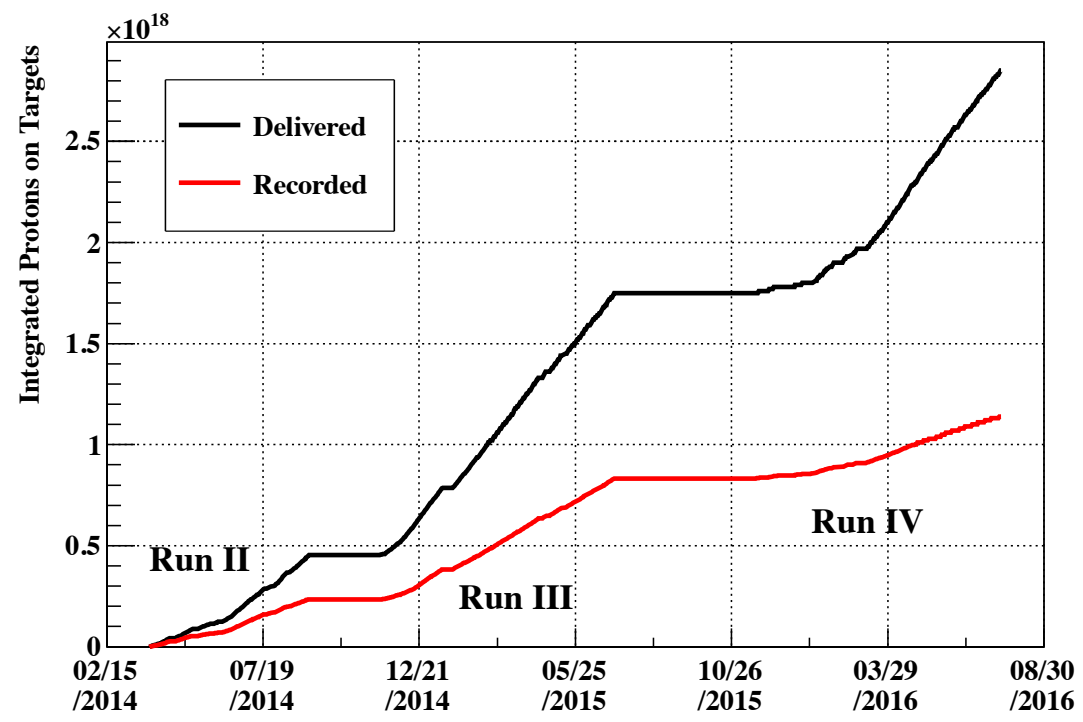

Figure 3.1: Integated protons on target. The black line shows the delivered protons on target, and the red line shows the recorded protons on target. 


\subsection{Proton Beam}

The SaeQuest uses a $120 \mathrm{GeV}$ proton beam. It is provided by the Fermilab Main Injector (FMI). The protons are accelerated up to $8 \mathrm{GeV}$ at LINAC and Booster, and then injected into FMI. Figure 3.2 shows the drawing of the FMI and the SeaQuest experimental hall.

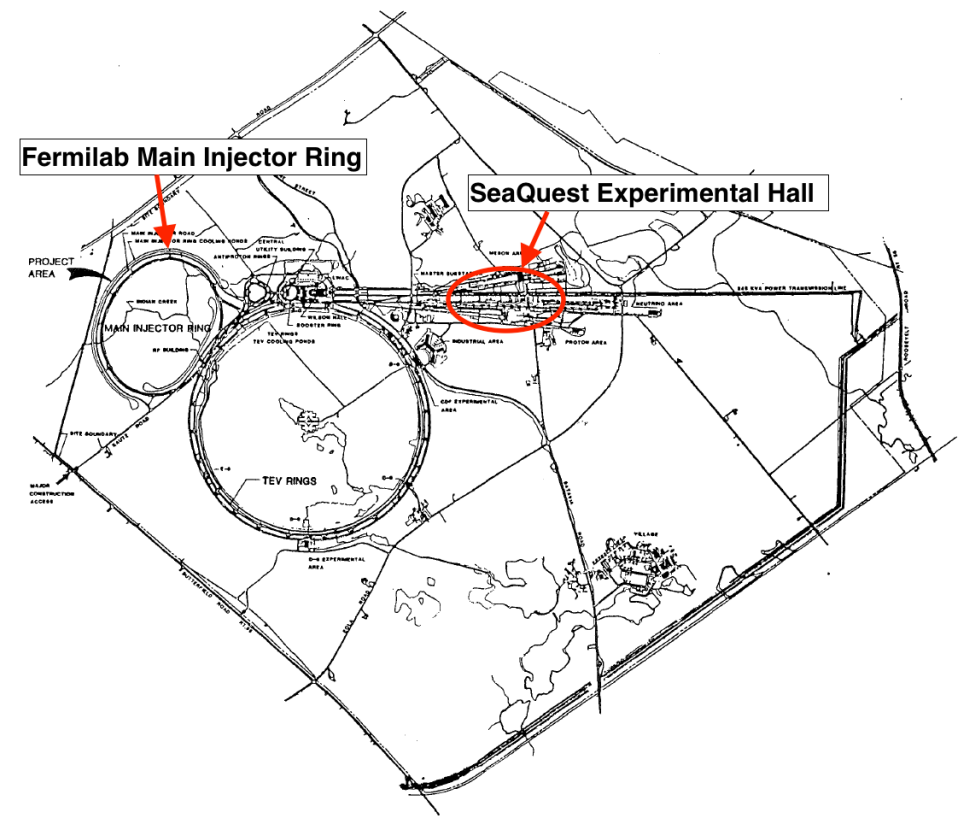

Figure 3.2: Drawing of Fermilab Main Injector and SeaQuest experimental hall [30].

Figure 3.3 shows the beam structure. The harmonic number of the Booster is 84 . The Booster

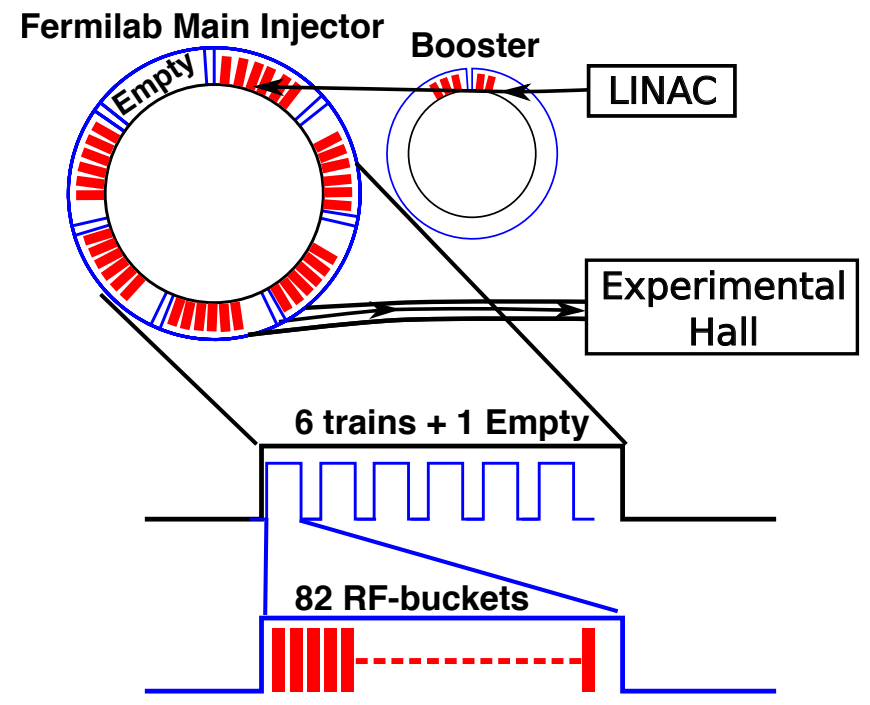

Figure 3.3: Beam structure of Fermilab Main Injector. Fermilab Main Injector holds six trans and 1 empty train. One train holds 492 RF-buckets.

can hold 84 beam bunches. A bunch is called "RF-bucket". RF stands for radio-frequency. The RF-bucket is 1-2 ns long. Typically, 82 out of 84 RF-buckets are filled with protons. A group of 84 RF-buckets is called a "train". The harmonic number of the FMI is 588, thus the FMI can hold 
seven trains. Six trains out of seven trains are in use. Therefore, FMI holds 492 RF-buckets which are filled with protons. The FMI accelerates the protons to $120 \mathrm{GeV}$. The accelerated proton beam is extracted by a slow extraction system. The system extracts about $4 \times 10^{4}$ protons. The proton beam is extracted every $\sim 19 \mathrm{~ns}(53 \mathrm{MHz})$ in each "spill". A spill is a five-second-long beam. It comes to experimental hall every minuite. The typical intensity of the spill is $10^{12}-10^{13}$ protons.

RF-signals are also provided from the FMI to the SeaQuest experimental hall. Since the SeaQuest uses the drift chambers, the timing information is very important for the measurement. The trigger system is synchronized with the provided FMI RF-signals.

\subsection{Beam Monitor}

It is important to monitor the proton beam intensity since the SeaQuest experiment measures the (relative) cross section of Drell-Yan process to extract $\bar{d} / \bar{u}$. In addition, the events are to be controlled so that the numbers of hits in detectors are not too high due to the high beam intensity. The beam monitor is also used in order to reject such kind of events.

SeaQuest uses three different monitors: ion chambers, SEM (secondary emission monitor), and Cherenkov counter for monitoring the number of protons. The ion chambers are used to give a feedback to the accelerator group, while the SEM and Cherenkov counter are mainly used for the analysis.

The Cherenkov counter is located upstream of the target. A diagram of the Cherenkov counter is shown in Fig. 3.4. The radiator of the Cherenkov counter is a gas with a mixture of $80 \%$ of Argon and

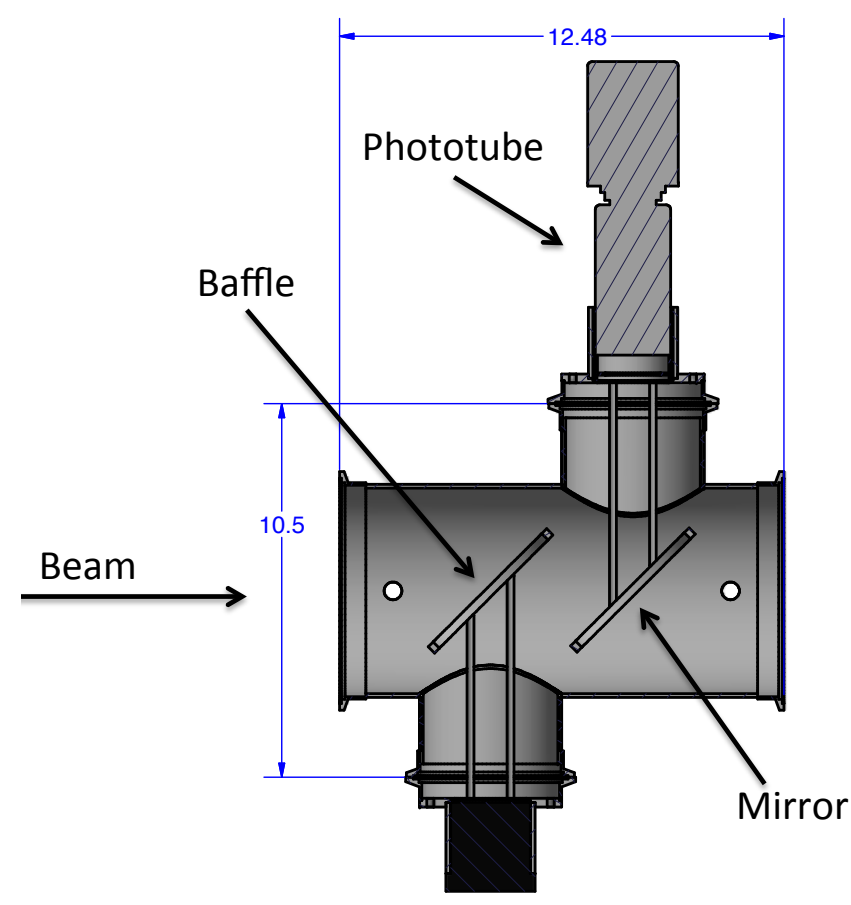

Figure 3.4: Cherenkov counter.

$20 \%$ of $\mathrm{CO}_{2}$. The mirror is made of Kapton and is aluminized. It directs the light to a photomultiplier tube (PMT). The signals from PMT is integrated and digitized with a "QIE" ("charge (Q) Integrator 
and Encoder") readout board. The readout system is synchronized with the FMI RF-signal. The advantage of the Cherenkov counter is the short response time. It can measure the number of protons in each $53 \mathrm{MHz}$ RF-bucket. It is also used as the trigger inhibitor. The inhibit threshold is set between 65,000 and 95,000 protons per RF-bucket. The measured value is not an absolute value of the proton intensity. This relative value of the integrated beam is called "QIE value". In order to measure the absolute number of protons in a RF-bucket, a measurement by the SEM is needed. The following information are provided by the Cherenkov counter:

- the integrated beam for entire spill (five seconds),

- the integrated beam while the trigger is inhibited due to the high intensity,

- the integrated beam during the trigger dead time, and

- the beams in RF-buckets which are the 16 RF-buckets before the triggered RF-buckets, the triggered RF-bucket, and 16 RF-buckets after the triggered RF-buckets.

The SEM is located upstream of the Cherenkov counter. The advantage of the SEM is to measure the absolute number of the beam protons while the Cherenkov counter measures relative value. However, the SEM is not able to measure the number of protons in each RF-bucket. It measures spill by spill. The role of the SEM is to measure the absolute number of protons in the entire spill. Combining the information from the Cherenkov counter and the SEM, the absolute number of the protons in each RF-bucket is extracted:

$$
N_{\mathrm{RF}}=Q_{\mathrm{RF}} \cdot \frac{N_{\text {Spill }}}{Q_{\text {Spill }}},
$$

where $N_{\mathrm{RF}}$ is the number of protons in a RF-bucket, $Q_{\mathrm{RF}}$ is the QIE value in a RF-bucket measured by the Cherenkov counter, $N_{\text {Spill }}$ is the number of protons in a spill measured by the SEM, and $Q_{\text {Spill }}$ is the QIE value in a spill measured by the Cherenkov counter. 


\subsection{Spectrometer}

Figure 3.5 shows the SeaQuest spectrometer. The SeaQuest spectrometer is 25 meter long. The spec-

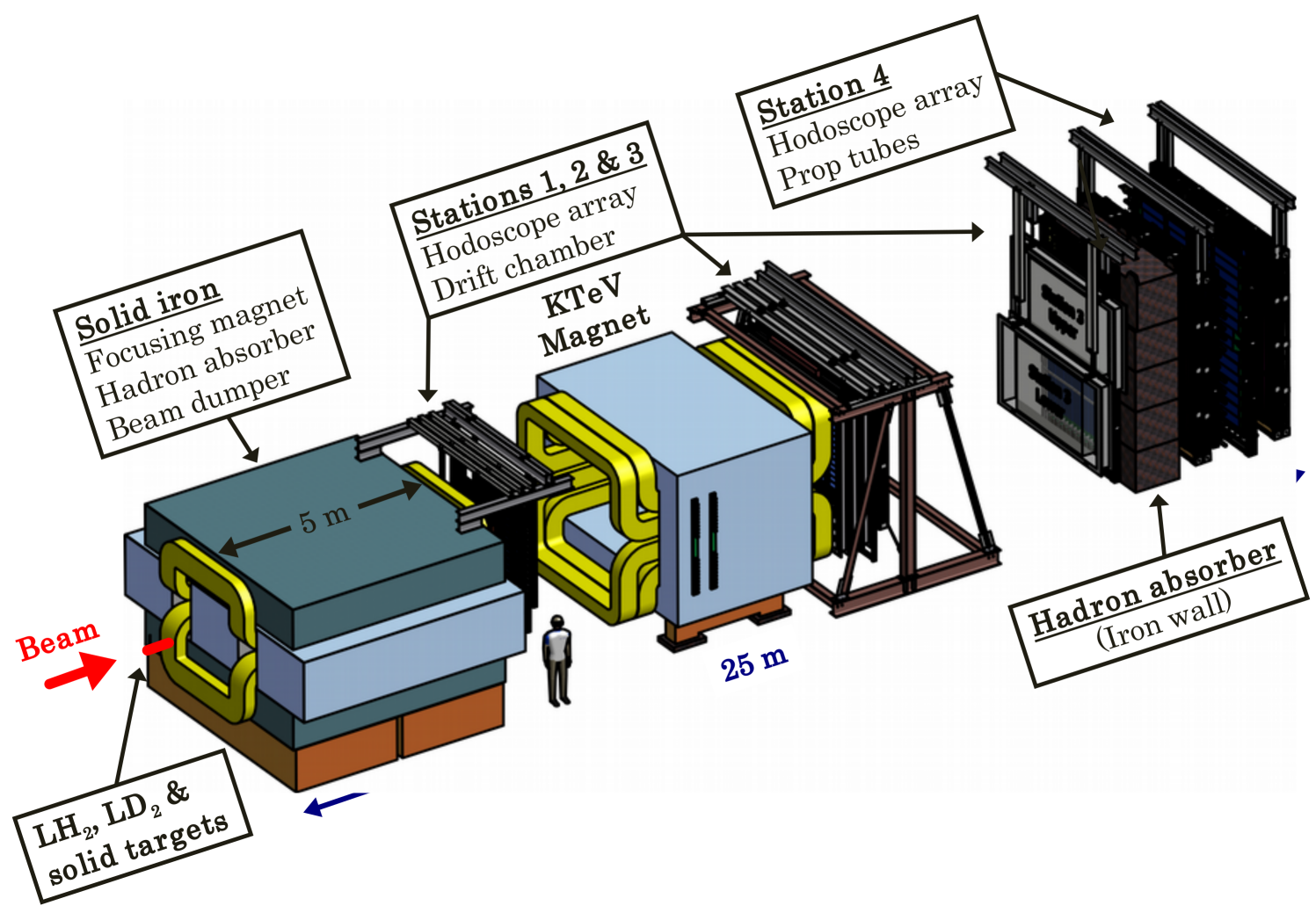

Figure 3.5: SeaQuest spectrometer. It consists of the targets, two dipole magnets, four tracking stations, and two hadron absorbers.

trometer consists of the targets, two dipole magnets, four tracking stations, and two hadron absorbers. The proton beam is injected from left-hand side of the figure and collides with the targets. A muon pair created from the Drell-Yan process passes through a focusing magnet which is also used as a hadron absorber. The muon pair is detected by four tracking stations. Another magnet is located between St. 1 and St. 2 in order to determine muon momenta. Another hadron absorber is located between St. 3 and St. 4 for the muon identification.

The $z$-axis of the laboratory frame is the direction of the proton beam. The $y$-axis is vertically upward. The $x$-axis is determined by the $z$ - and $y$-axes since the frame is the right-handed frame. All the axes are perpendicular to each other. $z=0$ corresponds to the upstream edge of the first magnet.

The details of the components of the spectrometer are explained in the following sub-sections.

\subsubsection{Targets}

SeaQuest uses two types of targets: liquid targets (Fig. 3.6 (a)) and solid targets (Fig. 3.6 (B)). Among them, the $\mathrm{LH}_{2}$ (hydrogen) and $\mathrm{LD}_{2}$ (deuterium) targets are used for measuring the antiquark flavor 


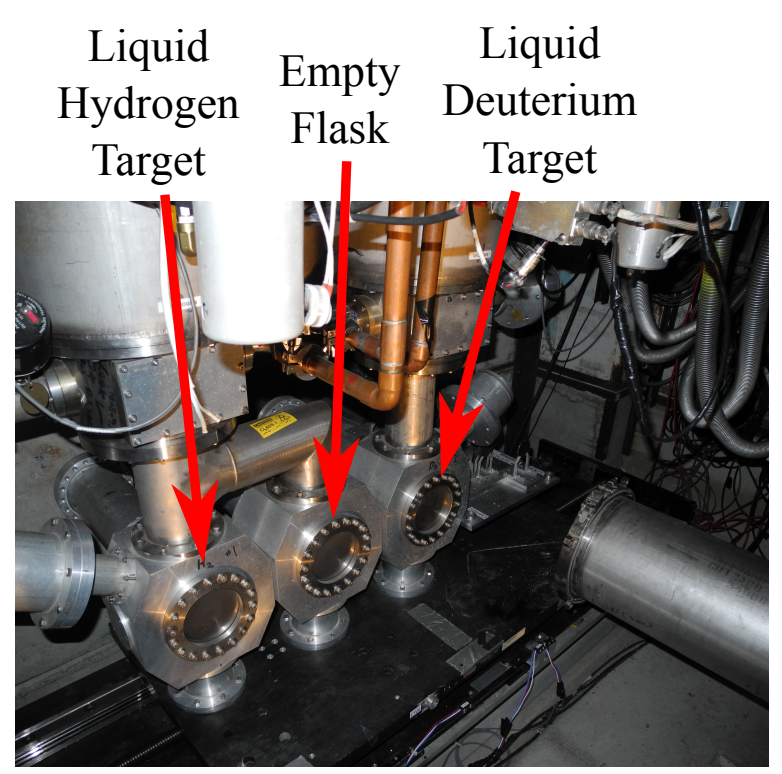

(a) Liquid targets $\left(\mathrm{LH}_{2}, \mathrm{LD}_{2}\right)$ and empty flask

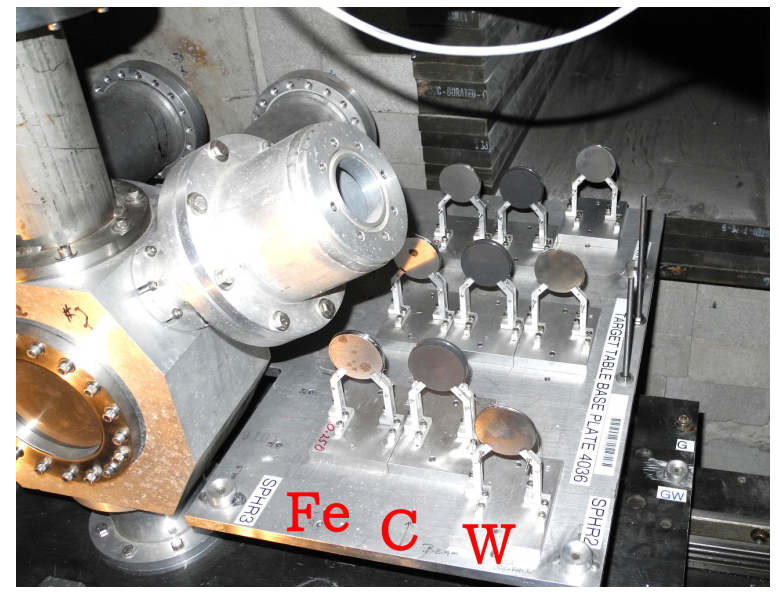

(b) Solid targets (iron, carbon, tungsten)

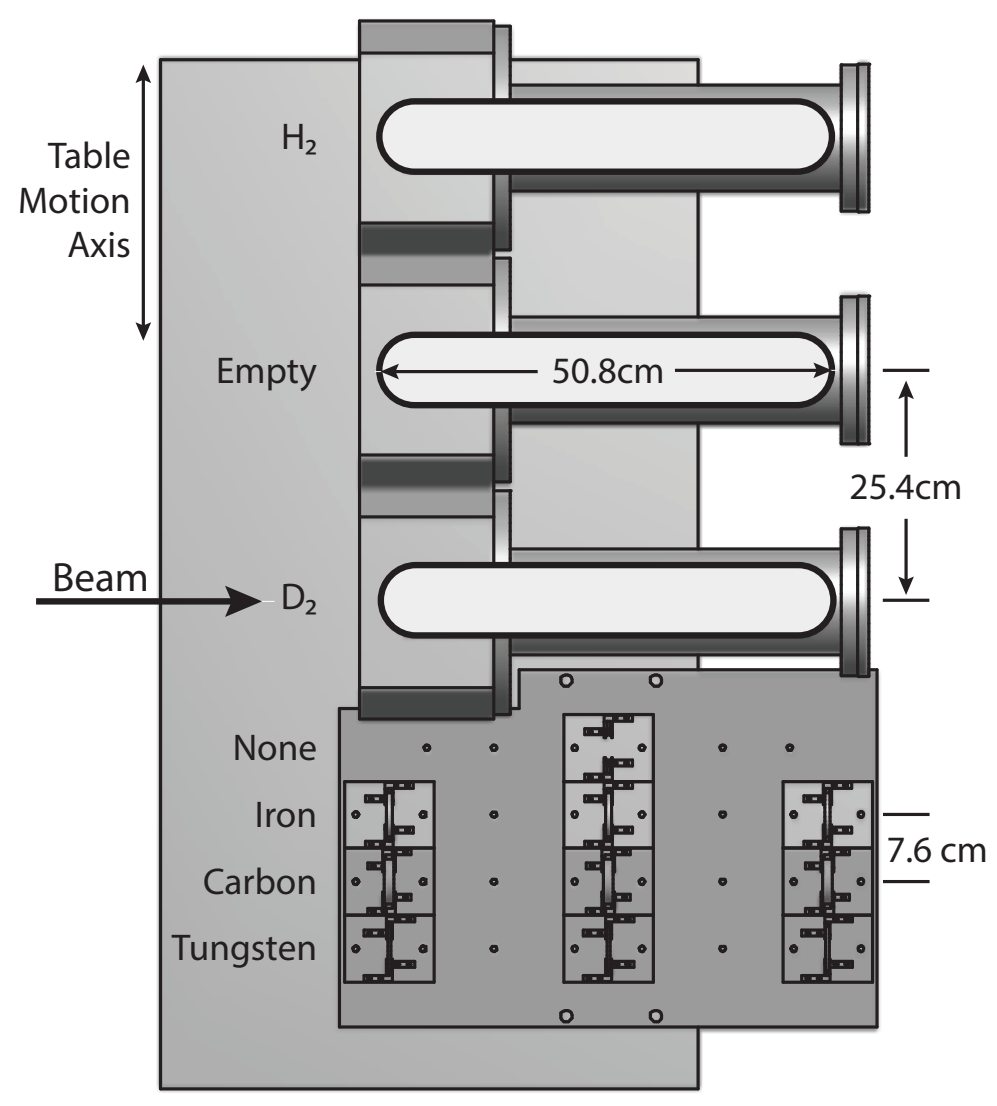

(c) Top view.

Figure 3.6: Targets used in the SeaQuest spectrometer. (a) $\mathrm{LH}_{2}$ and $\mathrm{LD}_{2}$ are used as the liquid targets. (b) Iron, carbon, tungsten are used as the solid targets. (c) Top view of all the targets on a movable table. 
asymmetry in the nucleon. The iron, carbon, and tungsten are the solid targets. They are used for measuring the nuclear effect. Each of the solids targets is divided into three disks so that the spectrometer acceptance approximates that of the liquid target. In addition, the empty flask target and none target data are also taken for background study. The targets are centered at $130 \mathrm{~cm}$ upstream of the first magnet of the spectrometer. Figure 3.6 (c) shows the top view of all the targets.

The $\mathrm{LH}_{2}$ target is $99.999 \%$ pure. The purity of $\mathrm{LD}_{2}$ target is about $90-95 \%$ in 2014 and in the former half of 2015. Later, SeaQuest switched to almost $100 \%$ pure $\mathrm{LD}_{2}$ source. Each target flask is surrounded by an insulation vacuum.

The targets are placed on a movable table. The table moves automatically between the spills to interchange the types of the targets. It takes about 30 seconds to interchange the targets, while the interval between the spills is 55 seconds. When it takes more than 45 seconds to interchange the targets due to some trouble in the target system, the inhibit signal goes off and the proton beam stops.

The target types and characteristics are summarized in Tab. 3.2.

Table 3.2: Types and characteristics of the SeaQuest targets.

\begin{tabular}{ccllrr}
\hline Position & Material & $\begin{array}{l}\text { Density } \\
\left(\mathrm{g} / \mathrm{cm}^{3}\right)\end{array}$ & $\begin{array}{l}\text { Thickness } \\
(\mathrm{cm})\end{array}$ & $\begin{array}{c}\text { Spills/Cycle } \\
(-2014)\end{array}$ & $\begin{array}{r}\text { Spills/Cycle } \\
(2015-)\end{array}$ \\
\hline 1 & $\mathrm{LH}_{2}$ & 0.071 & 50.8 & 10 & 10 \\
2 & Empty Flask & - & - & 1 & 2 \\
3 & $\mathrm{LD}_{2}$ & 0.163 & 50.8 & 5 & 5 \\
4 & None Target & - & - & 2 & 2 \\
5 & Iron & 7.87 & 1.905 & 1 & 1 \\
6 & Carbon & 1.80 & 3.322 & 3 & 2 \\
7 & Tungsten & 19.30 & 0.953 & 1 & 1 \\
\hline
\end{tabular}

\subsubsection{Magnets}

The SeaQuest spectrometer contains two dipole magnets. One is for focusing muons, and the other is for determination of the muon momenta. The direction of the magnetic field of the two magnet was $+y$ direction in 2014, but it was changed to $-y$ direction at the beginning of 2015 .

\subsubsection{FMag}

The most upstream magnet in the SeaQuest spectrometer is called FMag. Figure 3.7 is the drawing of the FMag. The FMag is assembled with $43.2 \mathrm{~cm} \times 160 \mathrm{~cm} \times 503 \mathrm{~cm}$ iron slabs. The excitation current is $2000 \mathrm{~A}$ at $25 \mathrm{~V}$ to generate a central magnetic field of $1.8 \mathrm{~T}$.

The FMag is used to select muons in appropriate momentum region. The low momentum muons are bent and are thrown out of the acceptance. It allows the high momentum muons to penetrate into the SeaQuest spectrometer. The FMag is also used as a beam dump. 

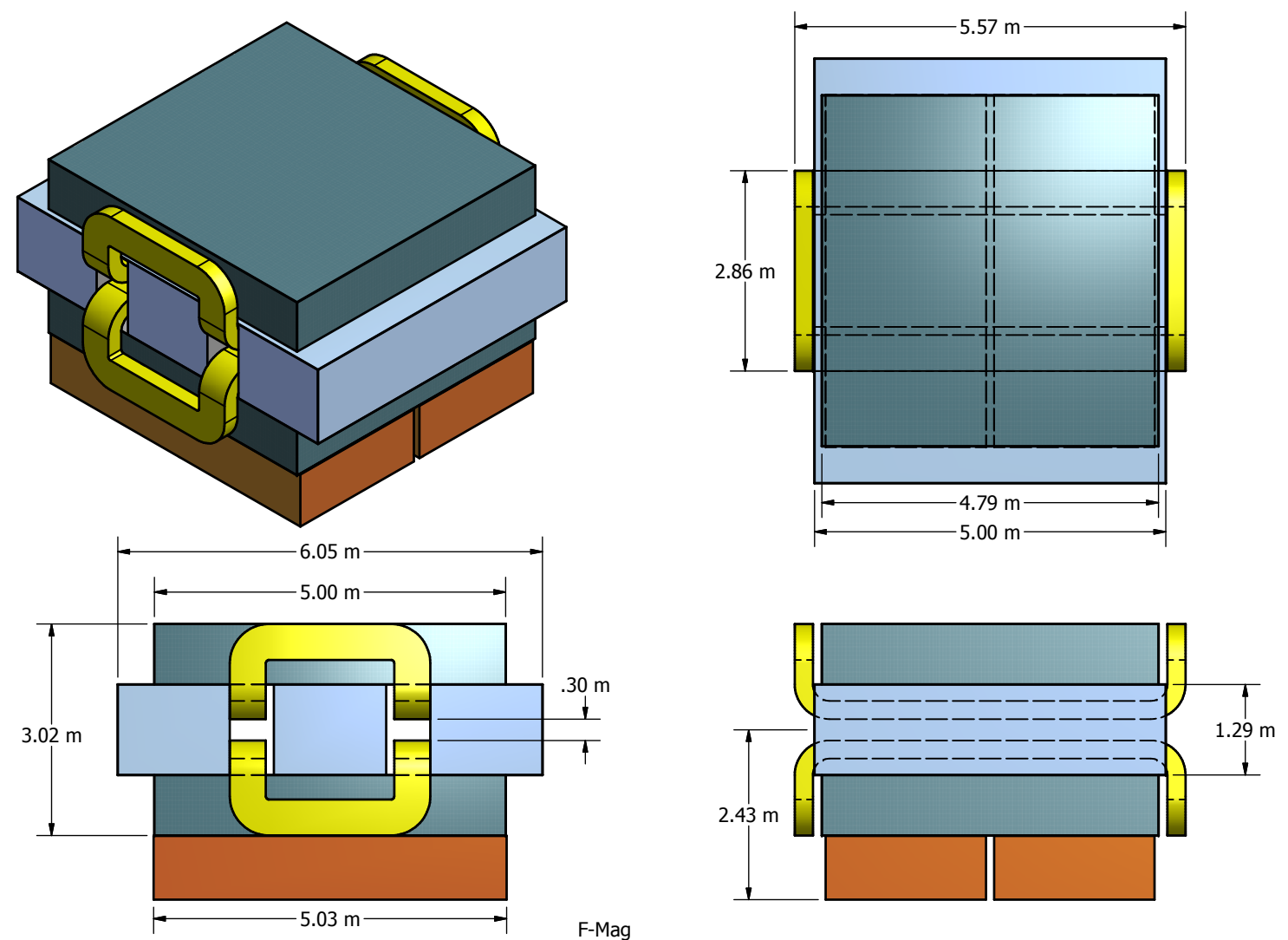

Figure 3.7: Drawing of the first dipole magnet FMag [31]. 
If the FMag current is low, too many particles penetrate into the spectrometer and may damage the detectors. The FMag current is monitored and input to the safety system in order to prevent too many particles from hitting the spectrometer.

\subsubsection{KMag}

The downstream magnet located between St. 1 and St. 2 is called KMag. This magnet is used for determination of muon momenta. An example of muon pair tracks are shown in Fig. 3.8. The muon

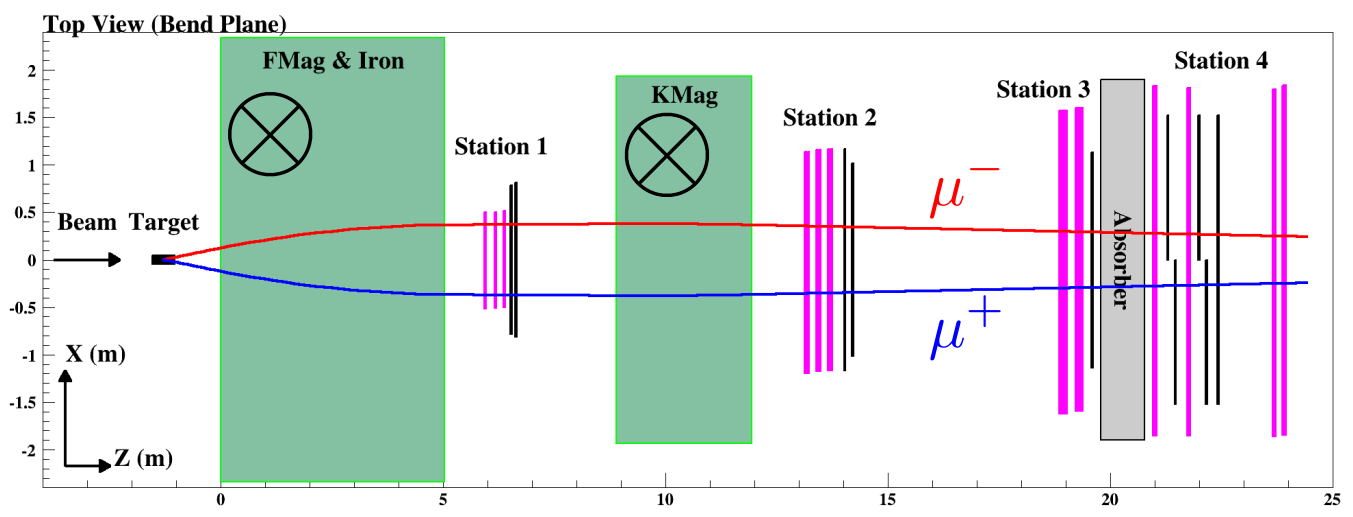

Figure 3.8: Top view of the detector and muon pair tracks. The red line is the track of $\mu^{+}$and the blue line is the track of $\mu^{-}$. The direction of the magnetic field is $-y$-direction.

momenta are evaluated from their curvature at KMag.

Figure 3.9 shows the drawing of KMag. The excitation current is $1600 \mathrm{~A}$ at $270 \mathrm{~V}$ to generate a central magnetic field of $0.4 \mathrm{~T}$. 

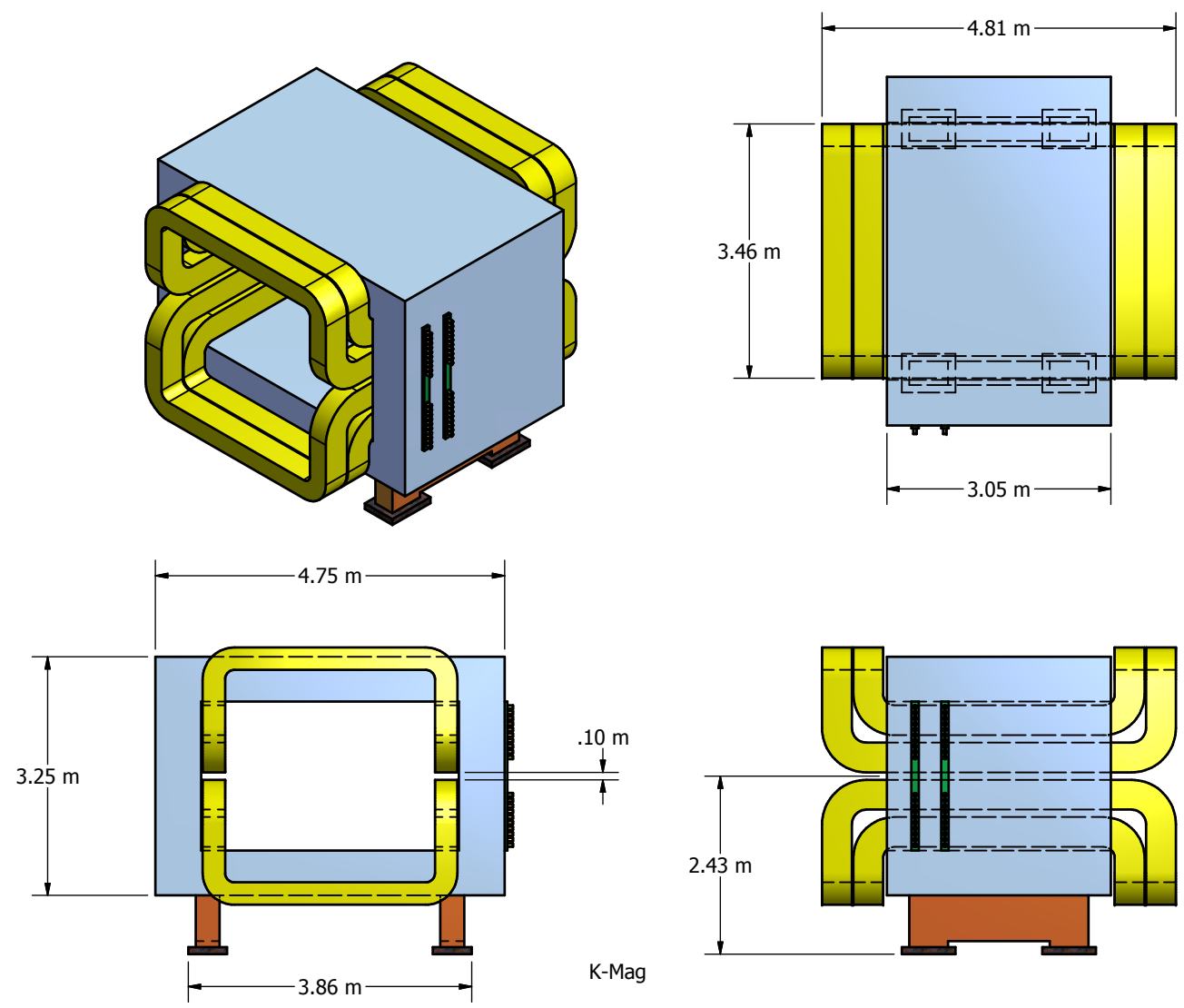

Figure 3.9: Drawing of the second dipole magnet KMag [31]. 


\subsubsection{Tracking Stations}

Four tracking stations are important elements in the SeaQuest spectrometer. Each St. 1 and St. 2 consists of a hodoscope array and a drift chamber. St. 3 should be wider and taller than St. 1 and St. 2 since the downstream station should cover larger acceptance than upstream once. It is, however, difficult to construct such a large drift chamber. So, the St. 3 drift chamber is separated into two parts: St. 3+ drift chamber for the upper half and St. 3- drift chamber for the lower half. Therefore, St. 3 consists of a hodoscope array and two drift chambers. St. 4 consists of a hodoscope array and proportional tubes.

\subsubsection{Hodoscopes}

Hodoscopes in the SeaQuest spectrometer are made of plastic scintillators. Each tracking station contains hodoscopes that are used as the primary trigger.

The hodoscope arrays are classified into "X-planes" and "Y-planes". The "X-planes" are arranged vertically to measure the $x$ position. The "Y-planes" are arranged horizontally to measure the $y$ position. Each St. 1, 2 and 4 contains both a X-plane and a Y-plane. St. 3 contains only a X-plane.

The number and size of the scintillators at each station are summarized in Tab. 3.3. The scintillator

Table 3.3: Number and size of scintillator at each hodoscope plane.

\begin{tabular}{lcccccc}
\hline Detector & $\begin{array}{c}\text { Width } \\
(\mathrm{cm})\end{array}$ & $\begin{array}{c}\text { Overlap } \\
(\mathrm{cm})\end{array}$ & $\begin{array}{c}\text { \# of } \\
\text { paddles }\end{array}$ & $\begin{array}{c}x \\
(\mathrm{~cm})\end{array}$ & $\begin{array}{c}y \\
(\mathrm{~cm})\end{array}$ & $\begin{array}{c}z \text {-position } \\
(\mathrm{cm})\end{array}$ \\
\hline H1T & 7.32 & 0.32 & 23 & 162.00 & 69.85 & 667.12 \\
H1B & 7.32 & 0.32 & 23 & 162.00 & 69.85 & 667.12 \\
H1L & 7.32 & 0.32 & 20 & 78.74 & 140.12 & 654.03 \\
H1R & 7.32 & 0.32 & 20 & 78.74 & 140.12 & 654.03 \\
H2T & 13.04 & 0.32 & 16 & 203.24 & 152.00 & 1421.06 \\
H2B & 13.04 & 0.32 & 16 & 203.24 & 152.00 & 1421.06 \\
H2L & 13.07 & 0.32 & 19 & 132.00 & 241.29 & 1402.86 \\
H2R & 13.07 & 0.32 & 19 & 132.00 & 241.29 & 1402.86 \\
H3T & 14.59 & 0.32 & 16 & 227.52 & 167.64 & 1958.51 \\
H3B & 14.59 & 0.32 & 16 & 227.52 & 167.64 & 1958.51 \\
H4T & 19.65 & 0.32 & 16 & 304.52 & 182.88 & 2234.50 \\
H4B & 19.65 & 0.32 & 16 & 304.52 & 182.88 & 2250.68 \\
H4Y1L & 23.48 & 0.32 & 16 & 152.40 & 365.80 & 2130.27 \\
H4Y1R & 23.48 & 0.32 & 16 & 152.40 & 365.80 & 2146.45 \\
H4Y2L & 23.48 & 0.32 & 16 & 152.40 & 365.80 & 2200.44 \\
H4Y2R & 23.48 & 0.32 & 16 & 152.40 & 365.80 & 2216.62 \\
\hline
\end{tabular}

paddles in X-plane (Y-plane) are split into top/bottom (left/right) paddles because the scintillators are long. The notations of "T", "B", "L" and "R" in Tab. 3.3 stand for top, bottom, left and right, 
respectively. The St. 4 scintillator paddles are read from both side because the hodoscopes are long due to the wide coverage. Each paddle is overlapped with the neighboring paddles by $2-3 \mathrm{~mm}$.

Figure 3.10-3.13 are the drawings of hodoscopes. 


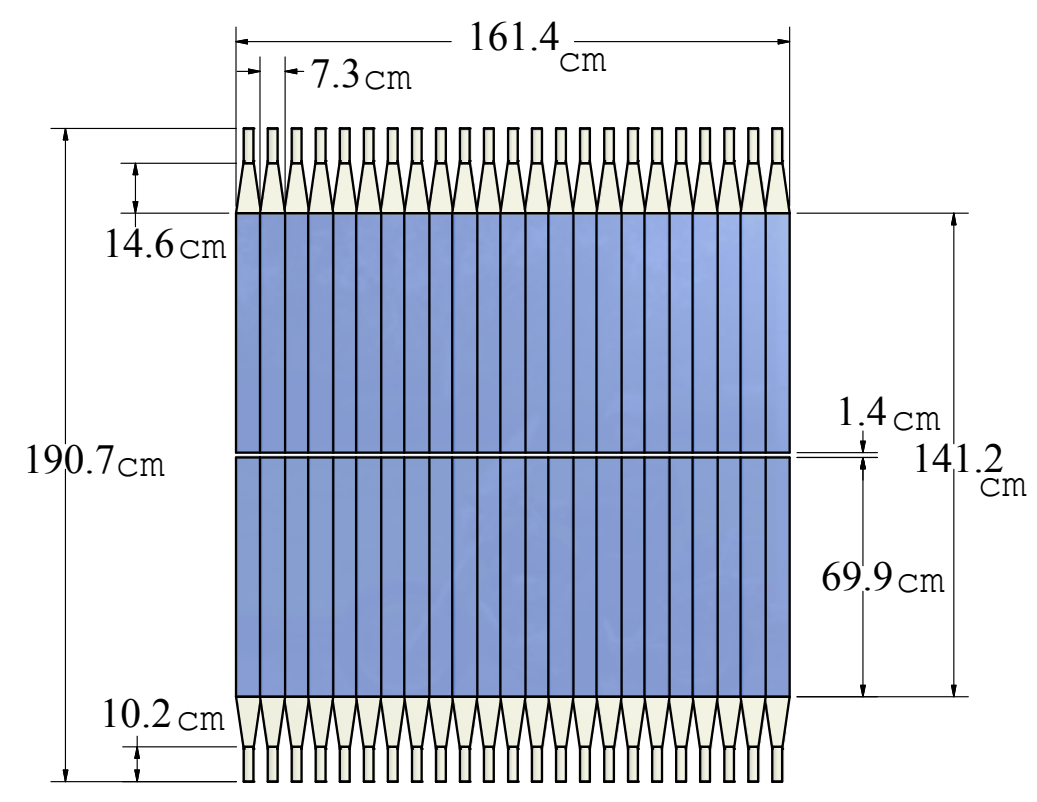

Hodoscope Array 1X

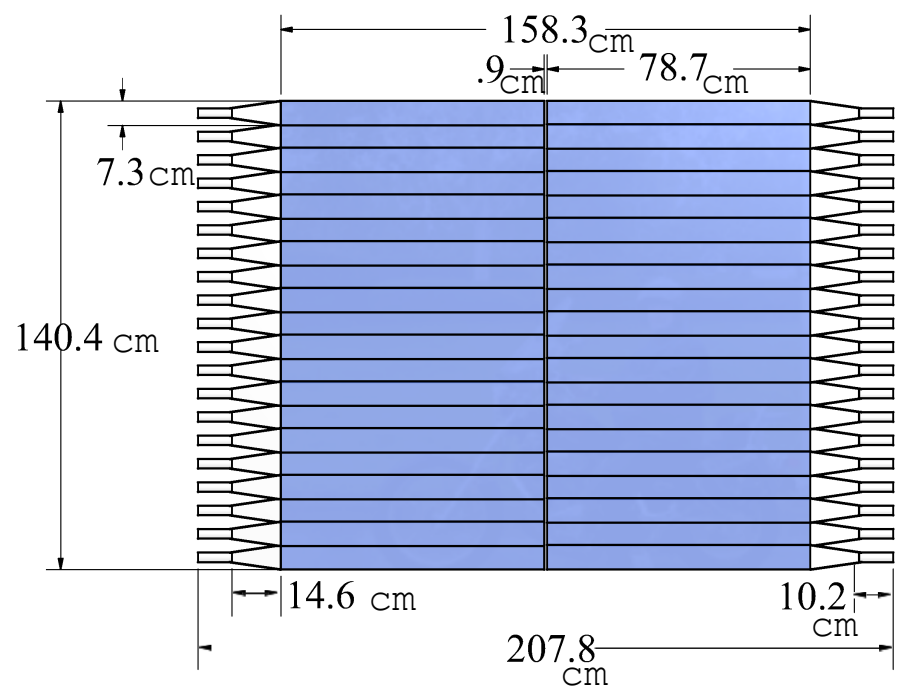

Hodoscope Array 1Y

Figure 3.10: Drawing of scintillator hodoscopes [31] of St. 1, named H1X, H1Y. The top figure shows the $\mathrm{X}$-plane, and the bottom figure shows the Y-plane. 

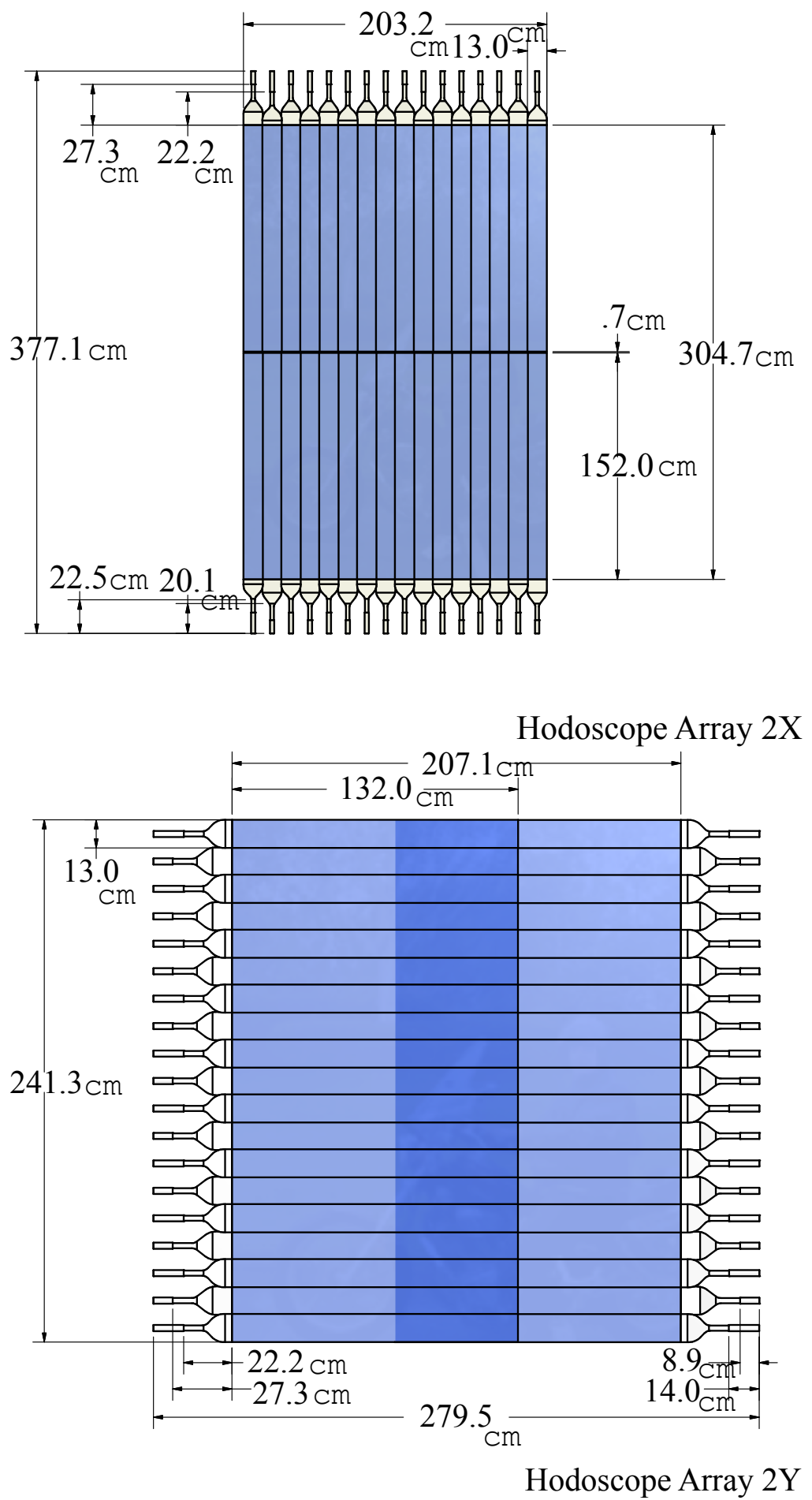

Figure 3.11: Drawing of scintillator hodoscopes [31] of St. 2, named H2X, H2Y. The top figure shows the $\mathrm{X}$-plane, and the bottom figure shows the Y-plane. The Y-planes of R and L overlap with each other by $57 \mathrm{~cm}$. 


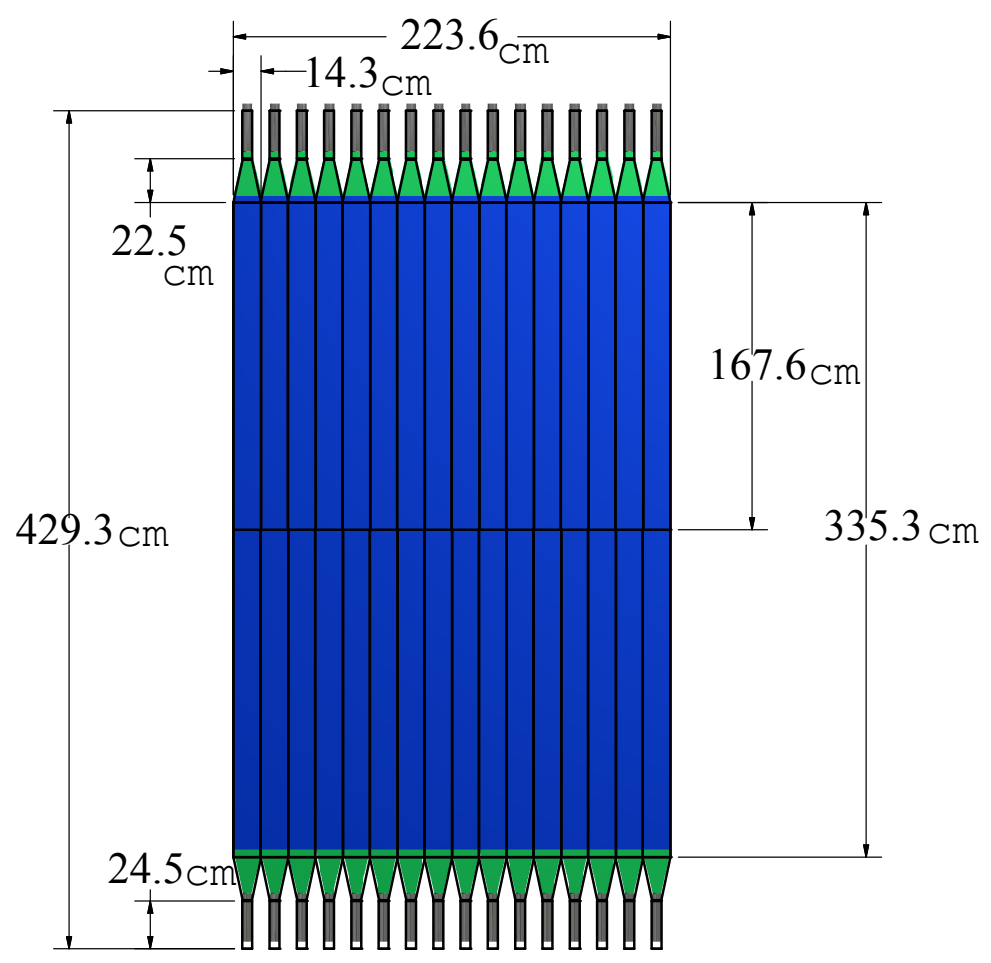

Hodoscope Array 3X

Figure 3.12: Drawing of scintillator hodoscopes [31] of St. 3, named H3X. The St. 3 contains only the X-plane. 

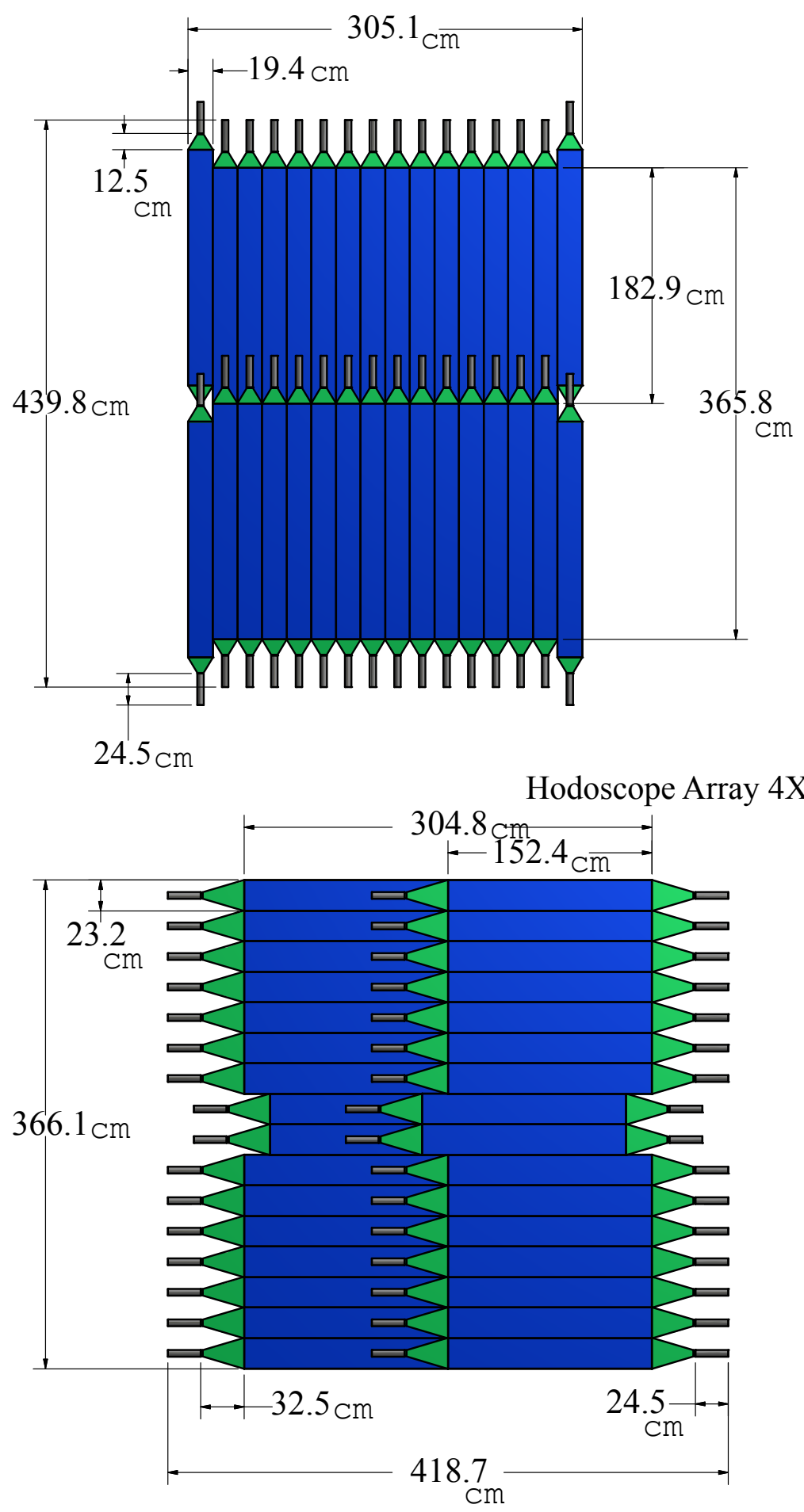

Hodoscope Array 4Y

Figure 3.13: Drawing of scintillator hodoscopes [31] of St. 4, named H4X, H4Y. The top figure shows the $\mathrm{X}$-plane, and the bottom figure shows the Y-plane. The St. 4 hodoscopes are read from both sides: from top and bottom for X-plane and from left and right for Y-plane. 


\subsubsection{Drift Chambers}

As mentioned in subsection 3.3.3, each of St. 1 and St. 2 has one drift chamber (D1 for St. 1, D2 for St. 2) and St. 3 has two drift chambers (D3p for St. 3+, D3m for St. 3-). In total, four drift chambers are located in the SeaQuest spectrometer. Each drift chamber has six planes: namely $\mathrm{X}, \mathrm{X}^{\prime}, \mathrm{V}, \mathrm{V}^{\prime}$, $\mathrm{U}$ and $\mathrm{U}^{\prime}$. The wire direction of $\mathrm{X}$ and $\mathrm{X}^{\prime}$ is vertical, and the wire direction of $\mathrm{U}, \mathrm{U}^{\prime}, \mathrm{V}$ and $\mathrm{V}^{\prime}$ is tilted by only +14 or -14 degrees from the vertical direction. That is because the $x$ measurement is more important as the muons are bent in the horizontal plane at KMag. The wires in primed planes are shifted by a half cell width with respect to the wires in un-primed planes in order to resolve the left-right ambiguity in the track reconstruction. The gas mixture used for the drift chambers is Argon:Methane: $\mathrm{CF}_{4}(88: 8: 4)$.

The D1 and D2 were used in the previous experiment at Fermilab, E866 and E605, respectively. The D3p and D3m were designed for the SeaQuest experiment for covering the wider acceptance. The D3p was constructed in Japan and shipped to Fermilab. The D3m was constructed in Fermilab under the initiative of the Japanese group.

Table 3.4 shows the parameters of all the drift chambers. Figures 3.14-3.17 show the structure of the drift chambers.

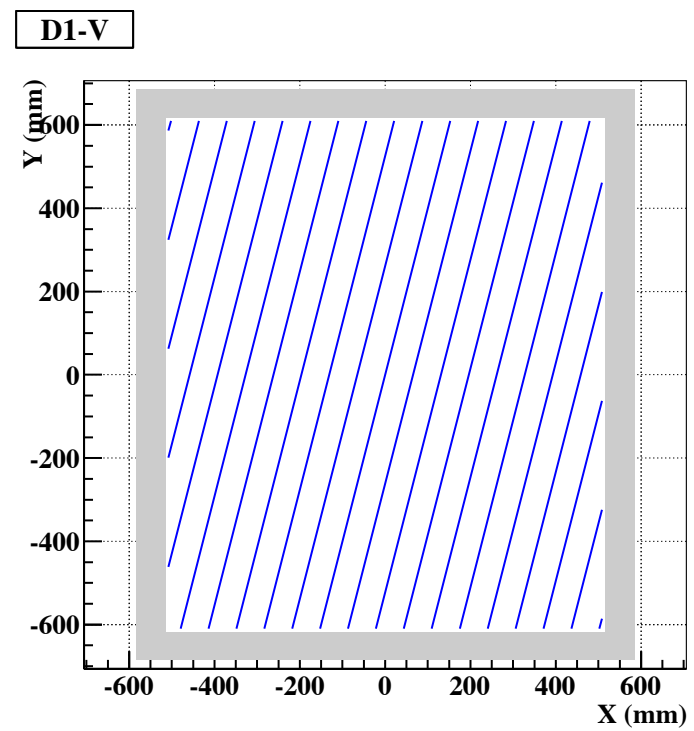

(a) V plane

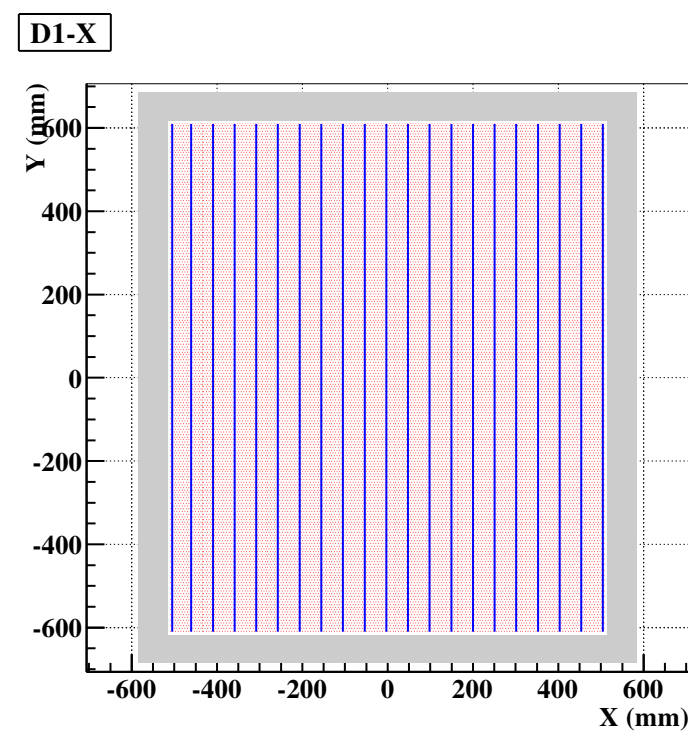

(b) X plane

Figure 3.14: Structure of St. 1 drift chamber. The V plane is shown in (a). The wires are tilted by about $14^{\circ}$ from vertical direction. The $\mathrm{X}$ plane is shown in (b). The wires are vertical. 
Table 3.4: Parameters of all the drift chambers.

\begin{tabular}{llllllr}
\hline Detector & $\begin{array}{l}\text { cell size } \\
(\mathrm{cm})\end{array}$ & \# of cells & $\begin{array}{l}\text { tilt angle } \\
(\mathrm{rad})\end{array}$ & $\begin{array}{l}\text { width } \\
(\mathrm{cm})\end{array}$ & $\begin{array}{l}\text { height } \\
(\mathrm{cm})\end{array}$ & $\begin{array}{r}z \text {-position } \\
(\mathrm{cm})\end{array}$ \\
\hline D1U & 0.635 & 201 & 0.245 & 101.60 & 121.92 & 594.49 \\
D1Up & 0.635 & 201 & 0.245 & 101.60 & 121.92 & 595.13 \\
D1X & 0.635 & 160 & 0 & 101.60 & 121.92 & 617.09 \\
D1Xp & 0.635 & 160 & 0 & 101.60 & 121.92 & 617.72 \\
D1V & 0.635 & 201 & -0.245 & 101.60 & 121.92 & 637.17 \\
D1Vp & 0.635 & 201 & -0.245 & 101.60 & 121.92 & 637.81 \\
\hline D2V & 2.021 & 128 & -0.245 & 233.27 & 264.16 & 1314.98 \\
D2Vp & 2.021 & 128 & -0.245 & 233.27 & 264.16 & 1321.96 \\
D2Xp & 2.083 & 112 & 0 & 233.27 & 264.16 & 1340.36 \\
D2X & 2.083 & 112 & 0 & 233.27 & 264.16 & 1347.34 \\
D2U & 2.021 & 128 & 0.245 & 233.27 & 264.16 & 1365.99 \\
D2Up & 2.021 & 128 & 0.245 & 233.27 & 264.16 & 1372.98 \\
\hline D3pVp & 2.000 & 134 & 0.245 & 320.00 & 166.00 & 1923.33 \\
D3pV & 2.000 & 134 & 0.245 & 320.00 & 166.00 & 1925.33 \\
D3pXp & 2.000 & 116 & 0 & 320.00 & 166.00 & 1929.33 \\
D3pX & 2.000 & 116 & 0 & 320.00 & 166.00 & 1931.33 \\
D3pUp & 2.000 & 134 & -0.245 & 320.00 & 166.00 & 1935.33 \\
D3pU & 2.000 & 134 & -0.245 & 320.00 & 166.00 & 1937.33 \\
\hline D3mVp & 2.000 & 134 & 0.245 & 320.00 & 166.00 & 1886.77 \\
D3mV & 2.000 & 134 & 0.245 & 320.00 & 166.00 & 1888.77 \\
D3mXp & 2.000 & 116 & 0 & 320.00 & 166.00 & 1892.77 \\
D3mX & 2.000 & 116 & 0 & 320.00 & 166.00 & 1894.77 \\
D3mUp & 2.000 & 134 & -0.245 & 320.00 & 166.00 & 1898.77 \\
D3mU & 2.000 & 134 & -0.245 & 320.00 & 166.00 & 1900.77 \\
\hline & & & & & &
\end{tabular}




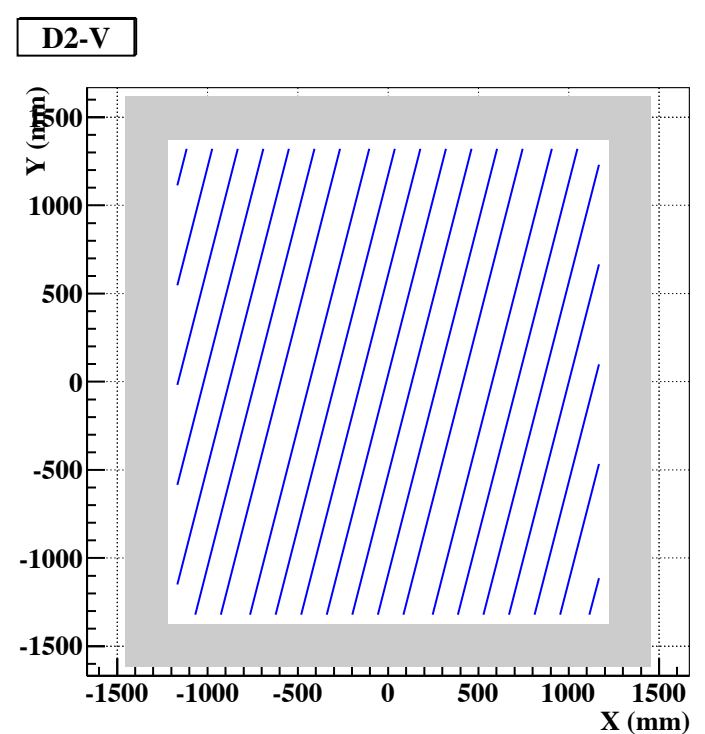

(a) V plane

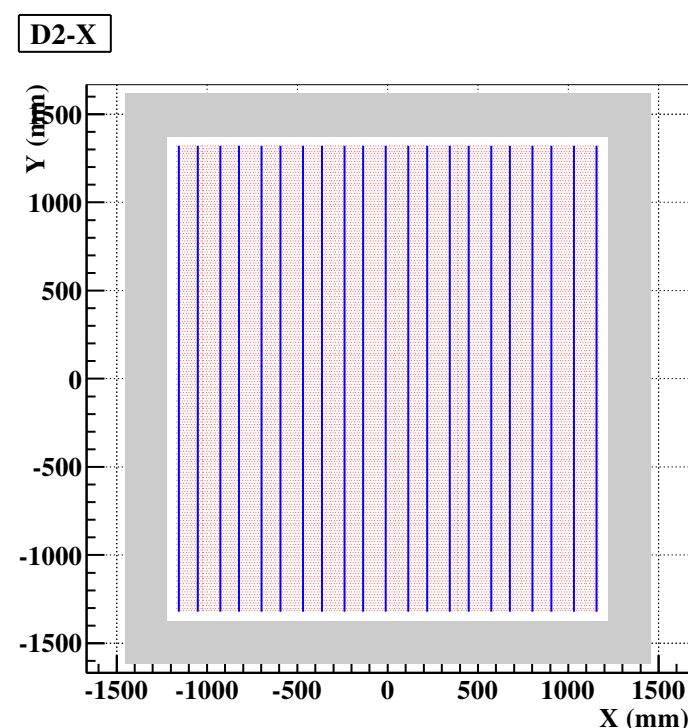

(b) X plane

Figure 3.15: Structure of St. 2 drift chamber. The V plane is shown in (a). The wires are tilted by about $14^{\circ}$ from vertical direction. The $\mathrm{X}$ plane is shown in (b). The wires are vertical.

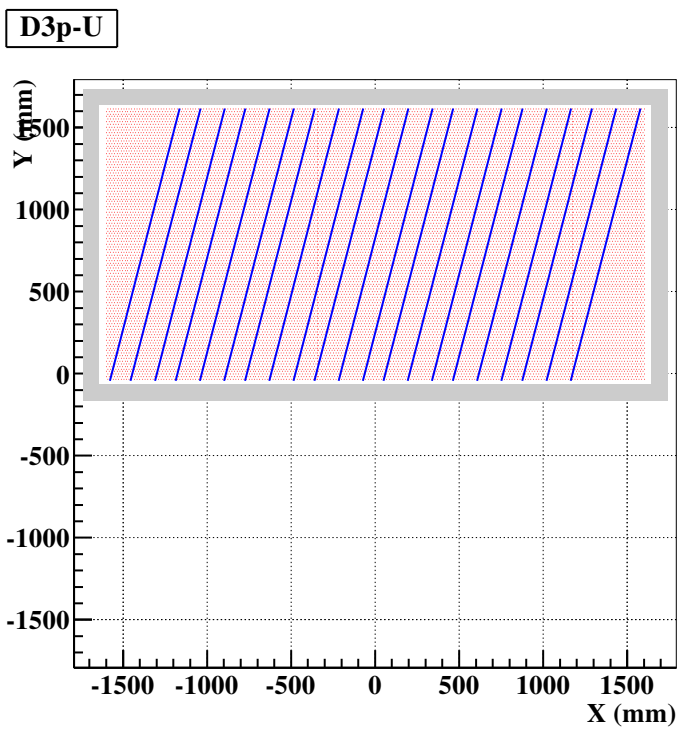

(a) U plane

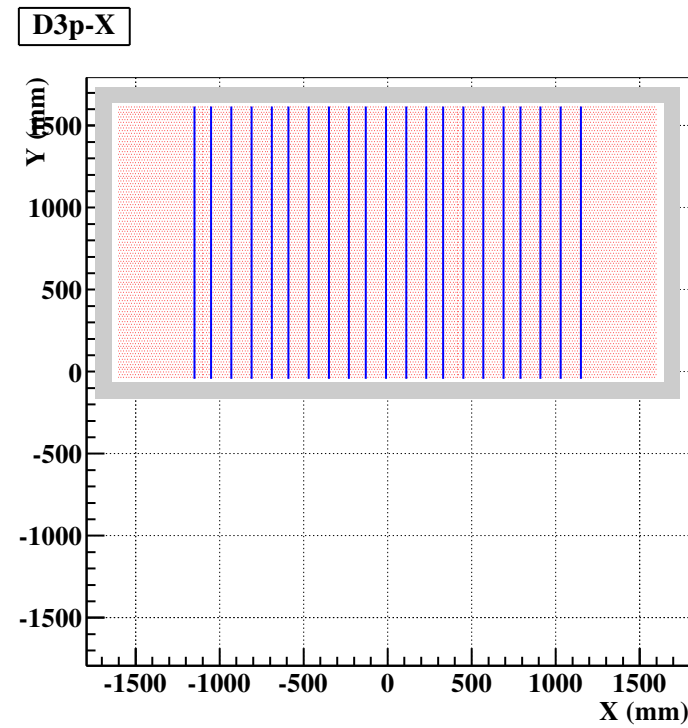

(b) X plane

Figure 3.16: Structure of St. 3+ drift chamber. The U plane is shown in (a). The wires are tilted by about $14^{\circ}$ from vertical direction. The $\mathrm{X}$ plane is shown in (b). The wires are vertical. The St. 3+ drift chamber covers upper half of St. 3. 


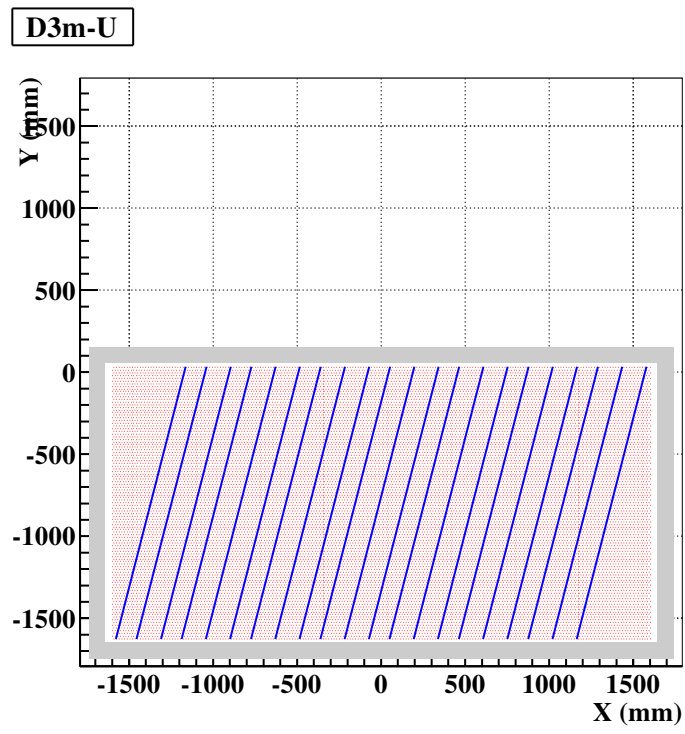

(a) U plane

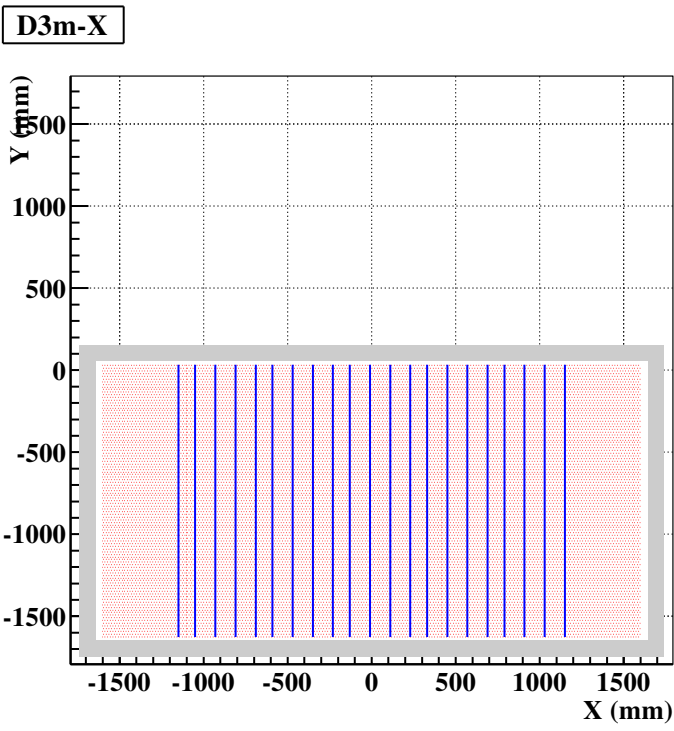

(b) X plane

Figure 3.17: Structure of St. 3- drift chamber. The U plane is shown in (a). The wires are tilted by about $14^{\circ}$ from vertical direction. The $\mathrm{X}$ plane is shown in (b). The wires are vertical. The St. 3- drift chamber covers lower half of St. 3. 
The cell structure of the St. 3 drift chamber is shown in Fig. 3.18. The wires are classified into

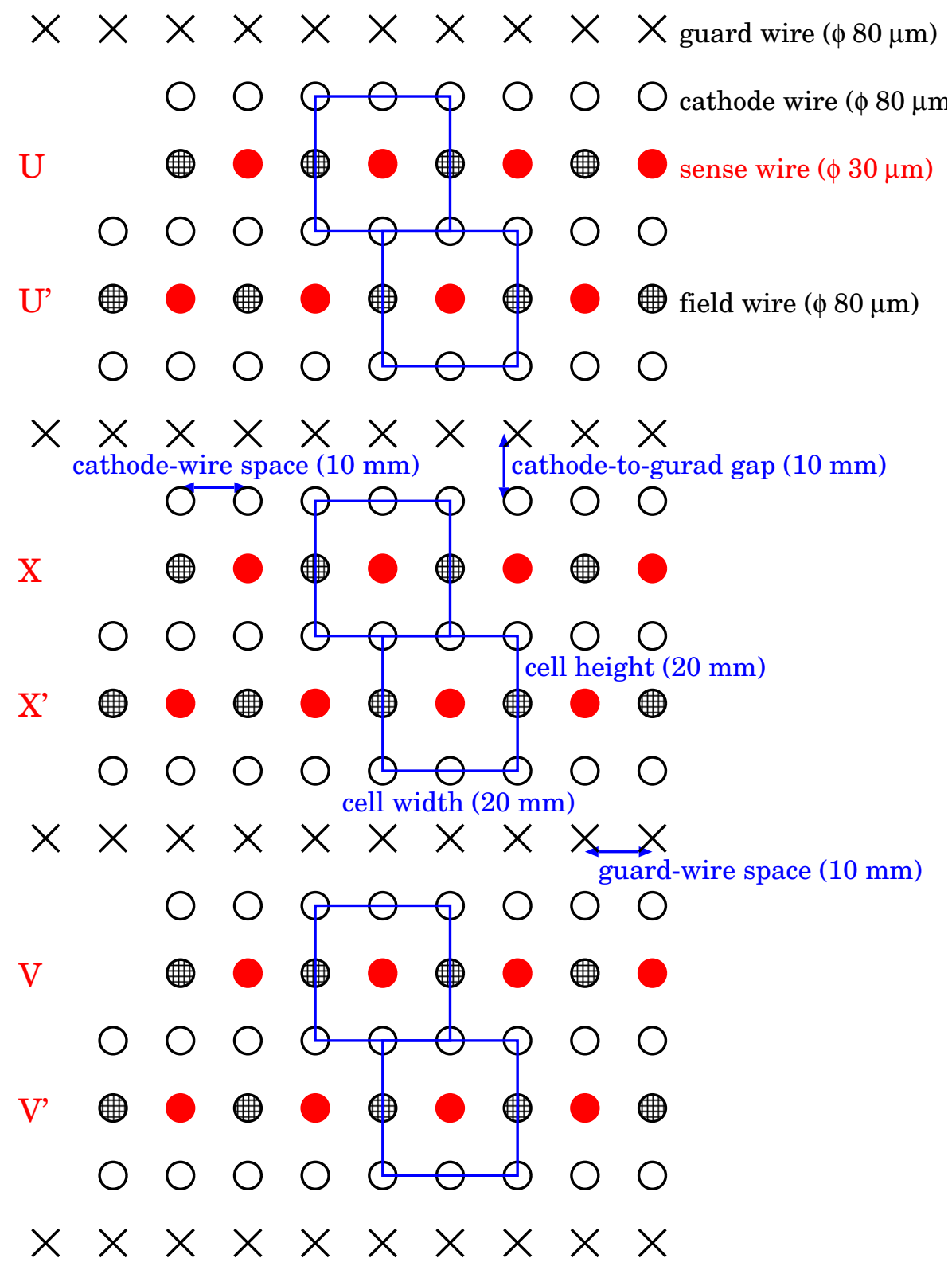

Figure 3.18: Cell structure of St. 3 drift chamber. The wires are classified into four types: sense wire (red circles), cathode wire (open circles), field wire (shaded circles), and guard wire (crosses).

four types: sense wires, cathode wires, field wires, and guard wires. Sense wires are grounded and are surrounded by potential wires which are cathode and field wires. The sense wire is $30 \mu \mathrm{m}$ diameter $\mathrm{Au}-\mathrm{W}$. The role of the sense wire is to read out the signal originating from the charged particles. The other wires are all the same types: $80 \mu \mathrm{m}$ diameter $\mathrm{Au}-\mathrm{BeCu}$. A voltage of about $-2400 \mathrm{~V}$ is applied to each potential wire and a voltage of about $-1500 \mathrm{~V}$ is applied to each guard wire. As the result, the electric field is generated between the sense wire and the potential wires. The electric potential between the wires is simulated with the Garfield simulation code [32] and is shown in Fig. 3.19. The sense wire is located at the center of the figure. The potential gradient around the sense wire is larger than the other location. It causes the electric avalanche effectively. 


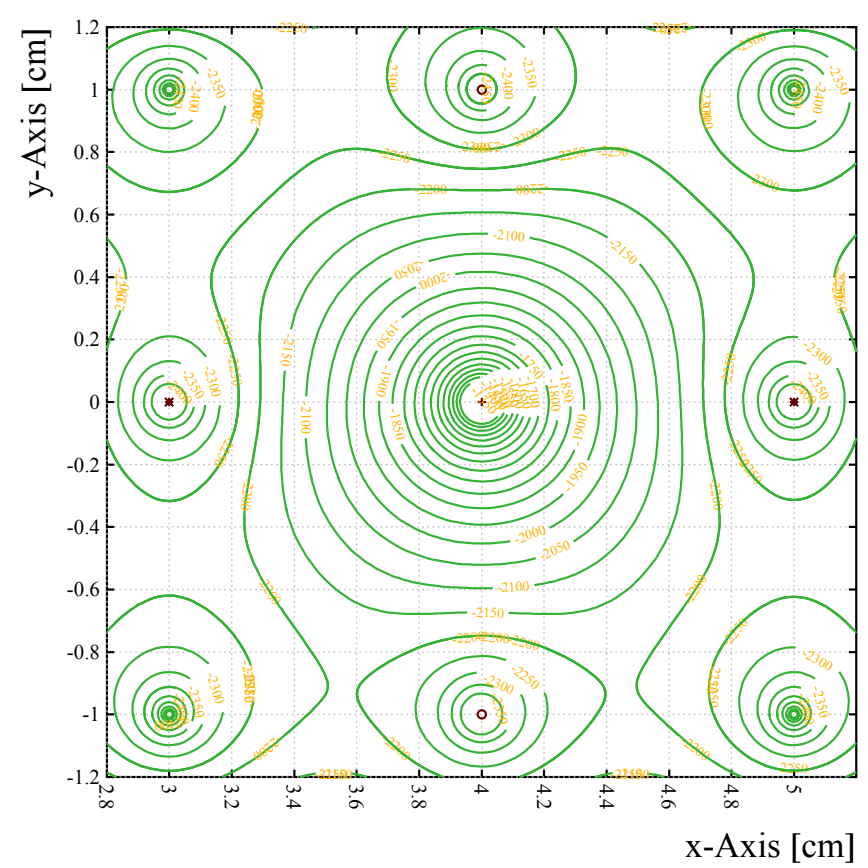

Figure 3.19: Electric potential between sense wire and field or cathode wires. The sense wire is located at the center of the figure. The open circles are cathode wires. The wires on the left and right sides of the sense wire are field wires.

The drift chamber detects the drift time of the electron. The drift time is converted to drift distance using RT-curve which shows the relation between the drift distance $(R)$ and drift time $(T)$. The drift distance is used for the track reconstruction. The track reconstruction, however, needs the drift distance of each chamber hit. An iterative analysis is performed starting with a rough estimate of initial RT-curve in order to obtain the precise RT-curve. Figure 3.20 shows the RT-curve after the iterative analysis.

The requirement of the plane detection efficiency $\left(\varepsilon_{\text {plane }}\right)$ is $>95 \%$ when the hit rate on the drift chamber is low. The tracking efficiency $\left(\varepsilon_{\mathrm{det}}\right)$ is $90 \%$ using this requirement with the following assumption: the definition of the track detection is that each drift chamber has at least five hits which are correlated with the track. It should be noted that there are six planes in each drift chamber.

$$
\begin{aligned}
\varepsilon_{\mathrm{det}} & =\varepsilon_{18}+\varepsilon_{17}+\varepsilon_{16}+\varepsilon_{15}, \\
& \left\{\begin{array}{l}
\varepsilon_{18}=\varepsilon_{6}^{3} \\
\varepsilon_{17}=\varepsilon_{6}^{2} \cdot \varepsilon_{5} \cdot 3 \\
\varepsilon_{16}=\varepsilon_{6} \cdot \varepsilon_{5}^{2} \cdot 3 \\
\varepsilon_{15}=\varepsilon_{5}^{3}
\end{array}\right. \\
& \left\{\begin{array}{l}
\varepsilon_{6}=\varepsilon_{\text {plane }}^{6} \\
\varepsilon_{5}=\varepsilon_{\text {plane }}^{5} \cdot\left(1-\varepsilon_{\text {plane }}\right) \cdot 6
\end{array},\right.
\end{aligned}
$$

where $\varepsilon_{18-15}$ are the tracking efficiencies when the number of detected hits is $18-15$, respectively, and $\varepsilon_{6}$ and $\varepsilon_{5}$ are the tracking efficiency in one drift chamber when the number of detected hits in one 


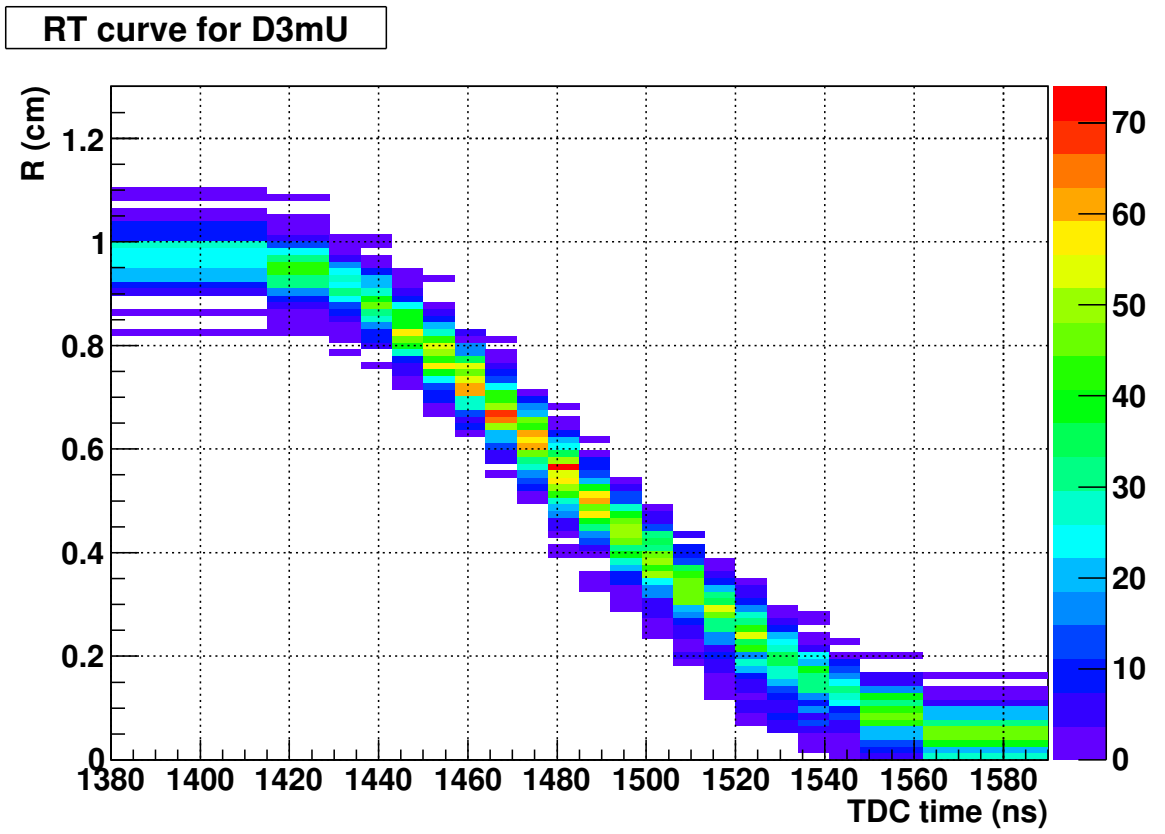

Figure 3.20: RT-curve of $\mathrm{U}$ plane on D3m. This is the result of the iterative analysis.

chamber is 6 and 5, respectively. The plane detection efficiency is measured with real data taken in 2014 [33]. The tracks are reconstructed by the SeaQuest official track reconstruction tool. (explained in Section 4.2). In addition to the track selections used in the data analysis (Section 4.3.2.1), the following selections are applied:

- $\chi^{2}<10$,

- number of hits associated with the track $\geq 17$.

$\chi^{2}$ is not reduced $\chi^{2}$ but $\chi^{2}$. The evaluation method of plane detection efficiency of D1X, for example, is shown here. Two kinds of tracks are used in this evaluation. One is "efficient track" which has 18 track-associated hits, and the other is "inefficient track" which doesn't have track-associated hit on $\mathrm{D} 1 \mathrm{X}$ and does have on all other planes. In order to ignore the effects of dead or noisy wires, if the track position on D1X is close to the known dead or noisy wires, the track is not used for efficiency evaluation. The regions which are regarded to be close to the dead or noisy wire are shown in Tab. 3.5. $w$ in Tab. 3.5 is defined as:

$$
w=x \cos \theta+y \sin \theta
$$

where $\theta$ is tilted angle of the wire, and $x$ and $y$ are the hit position at the plane. Using the numbers of the efficient and inefficient tracks, the plane detection efficiency is calculated as:

$$
\varepsilon_{\text {plane }}=\frac{N_{\text {eff }}}{N_{\text {eff }}+N_{\text {ineff }}},
$$

where $N_{\text {eff }}$ is the number of efficient tracks and $N_{\text {ineff }}$ is the number of inefficient tracks. The results of the plane detection efficiency evaluation are shown in Tab. 3.6. Most of them satisfy the requirement which is $95 \%$. The plane detection efficiency is lower than the requirement at left and right edges 
Table 3.5: List of the dead or noisy wire position.

\begin{tabular}{|c|c|}
\hline Plane & Position (w) \\
\hline $\mathrm{D} 1 \mathrm{U}$ & $-2 \mathrm{~cm} \mathrm{-}$ \\
\hline D1X & $-6 \mathrm{~cm}-$ \\
\hline D1X & $1 \mathrm{~cm} \mathrm{-}$ \\
\hline D1X & $43 \mathrm{~cm} \mathrm{-}$ \\
\hline D1Xp & $-7 \mathrm{~cm} \mathrm{-} \quad-3 \mathrm{~cm}$ \\
\hline D1Xp & $5 \mathrm{~cm} \mathrm{-}$ \\
\hline D1Xp & $17 \mathrm{~cm} \mathrm{-}$ \\
\hline D1Xp & $41 \mathrm{~cm} \mathrm{-}$ \\
\hline D1V & $-11 \mathrm{~cm} \mathrm{-} \quad-8 \mathrm{~cm}$ \\
\hline D1Vp & $-46 \mathrm{~cm} \mathrm{-}-40 \mathrm{~cm}$ \\
\hline $\mathrm{D} 1 \mathrm{Vp}$ & $-11 \mathrm{~cm} \mathrm{-} \quad-9 \mathrm{~cm}$ \\
\hline D1Vp & $32 \mathrm{~cm} \mathrm{-}$ \\
\hline $\mathrm{D} 1 \mathrm{Vp}$ & $46 \mathrm{~cm} \mathrm{-}$ \\
\hline $\mathrm{D} 2 \mathrm{Vp}$ & $65 \mathrm{~cm} \mathrm{-}$ \\
\hline D2Xp & $-50 \mathrm{~cm} \mathrm{-}-40 \mathrm{~cm}$ \\
\hline $\mathrm{D} 2 \mathrm{Xp}$ & $-12 \mathrm{~cm} \mathrm{-} \quad-5 \mathrm{~cm}$ \\
\hline $\mathrm{D} 2 \mathrm{Xp}$ & $105 \mathrm{~cm} \mathrm{-} 110 \mathrm{~cm}$ \\
\hline $\mathrm{D} 2 \mathrm{X}$ & $-50 \mathrm{~cm}-\quad-40 \mathrm{~cm}$ \\
\hline $\mathrm{D} 2 \mathrm{X}$ & $-20 \mathrm{~cm} \mathrm{-}$ \\
\hline $\mathrm{D} 2 \mathrm{U}$ & $105 \mathrm{~cm} \mathrm{-} 110 \mathrm{~cm}$ \\
\hline D2Up & $-20 \mathrm{~cm} \mathrm{-}-12 \mathrm{~cm}$ \\
\hline D2Up & $45 \mathrm{~cm} \mathrm{-}$ \\
\hline $\mathrm{D} 3 \mathrm{pVp}$ & $-120 \mathrm{~cm} \mathrm{-}-90 \mathrm{~cm}$ \\
\hline D3pV & $-120 \mathrm{~cm} \mathrm{-}-85 \mathrm{~cm}$ \\
\hline D3pV & $140 \mathrm{~cm} \mathrm{-} \quad 150 \mathrm{~cm}$ \\
\hline $\mathrm{D} 3 \mathrm{pXp}$ & $-120 \mathrm{~cm}--100 \mathrm{~cm}$ \\
\hline D3pX & $-120 \mathrm{~cm} \mathrm{-}-85 \mathrm{~cm}$ \\
\hline D3pX & $60 \mathrm{~cm} \mathrm{-}$ \\
\hline D3pUp & $-150 \mathrm{~cm}--130 \mathrm{~cm}$ \\
\hline D3pUp & $35 \mathrm{~cm} \mathrm{-}$ \\
\hline D3pU & $-150 \mathrm{~cm}--125 \mathrm{~cm}$ \\
\hline D3pU & $30 \mathrm{~cm} \mathrm{-}$ \\
\hline
\end{tabular}


Table 3.6: Plane detection efficiency of each drift chamber plane.

\begin{tabular}{l|c|c|c}
\hline Plane & Edge (Left) & Center & Edge (Right) \\
\hline \hline D1U & $90.0 \pm 1.6 \%$ & $98.0 \pm 0.0 \%$ & $92.9 \pm 0.8 \%$ \\
D1Up & $88.1 \pm 1.7 \%$ & $98.3 \pm 0.0 \%$ & $91.9 \pm 0.8 \%$ \\
D1X & $81.2 \pm 2.0 \%$ & $97.3 \pm 0.1 \%$ & $87.8 \pm 1.0 \%$ \\
D1Xp & $82.4 \pm 2.0 \%$ & $97.5 \pm 0.1 \%$ & $89.5 \pm 0.9 \%$ \\
D1V & $92.2 \pm 2.1 \%$ & $98.7 \pm 0.0 \%$ & $94.4 \pm 0.7 \%$ \\
D1Vp & $92.6 \pm 1.3 \%$ & $98.4 \pm 0.0 \%$ & $93.7 \pm 0.8 \%$ \\
\hline D2V & $90.6 \pm 0.6 \%$ & $95.6 \pm 0.1 \%$ & $93.5 \pm 0.2 \%$ \\
D2Vp & $91.7 \pm 0.6 \%$ & $96.4 \pm 0.1 \%$ & $94.6 \pm 0.2 \%$ \\
D2Xp & $89.3 \pm 0.3 \%$ & $91.2 \pm 0.3 \%$ & $90.6 \pm 0.2 \%$ \\
D2X & $90.4 \pm 0.2 \%$ & $91.9 \pm 0.5 \%$ & $91.6 \pm 0.2 \%$ \\
D2U & $90.8 \pm 0.3 \%$ & $95.4 \pm 0.1 \%$ & $93.7 \pm 0.2 \%$ \\
D2Up & $90.6 \pm 0.3 \%$ & $95.9 \pm 0.1 \%$ & $94.4 \pm 0.2 \%$ \\
\hline D3pVp & $89.3 \pm 0.1 \%$ & $91.3 \pm 0.5 \%$ & $88.7 \pm 0.4 \%$ \\
D3pV & $90.8 \pm 0.1 \%$ & $92.6 \pm 0.4 \%$ & $89.4 \pm 0.4 \%$ \\
D3pXp & $91.5 \pm 0.1 \%$ & $93.1 \pm 0.5 \%$ & $91.5 \pm 0.2 \%$ \\
D3pX & $91.3 \pm 0.1 \%$ & $92.9 \pm 0.5 \%$ & $92.2 \pm 0.2 \%$ \\
D3pUp & $90.4 \pm 0.5 \%$ & $93.1 \pm 0.5 \%$ & $92.4 \pm 0.2 \%$ \\
D3pU & $90.3 \pm 0.3 \%$ & $92.4 \pm 0.4 \%$ & $90.9 \pm 0.2 \%$ \\
\hline D3mVp & $94.5 \pm 0.5 \%$ & $96.1 \pm 0.3 \%$ & $94.8 \pm 0.1 \%$ \\
D3mV & $93.8 \pm 0.5 \%$ & $97.0 \pm 0.3 \%$ & $95.4 \pm 0.1 \%$ \\
D3mXp & $94.3 \pm 0.1 \%$ & $95.4 \pm 0.4 \%$ & $94.3 \pm 0.1 \%$ \\
D3mX & $94.0 \pm 0.1 \%$ & $95.3 \pm 0.4 \%$ & $94.6 \pm 0.1 \%$ \\
D3mUp & $94.0 \pm 0.2 \%$ & $95.5 \pm 0.4 \%$ & $94.9 \pm 0.3 \%$ \\
D3mU & $93.3 \pm 0.2 \%$ & $94.2 \pm 0.4 \%$ & $93.7 \pm 0.3 \%$ \\
\hline
\end{tabular}


of the drift chambers. This is because the hit rate at the edge of drift chamber is higher than that at the center of the drift chamber. The efficiencies at most planes at the center are larger than the requirement. The efficiencies at some planes at the center are smaller than the requirement, but this is tolerable.

In this evaluation method, the tracks are reconstructed without ignoring the target plane. The difference between the efficiencies evaluated with track reconstruction ignoring the target plane and not ignoring the target plane is $\pm 2 \%$.

The requirement of the plane position resolution is $<400 \mu \mathrm{m}$ when the hit rate on the drift chamber is low. The plane position resolution is derived with the reconstructed tracks of the real data. The tracks are reconstructed by the SeaQuest official track reconstruction tool. The plane position resolution is defined as the residual of the drift distance and the track hit position at the plane. Figure 3.21 shows the residuals. There are two types of the residuals. One is evaluated with the tracks recon-
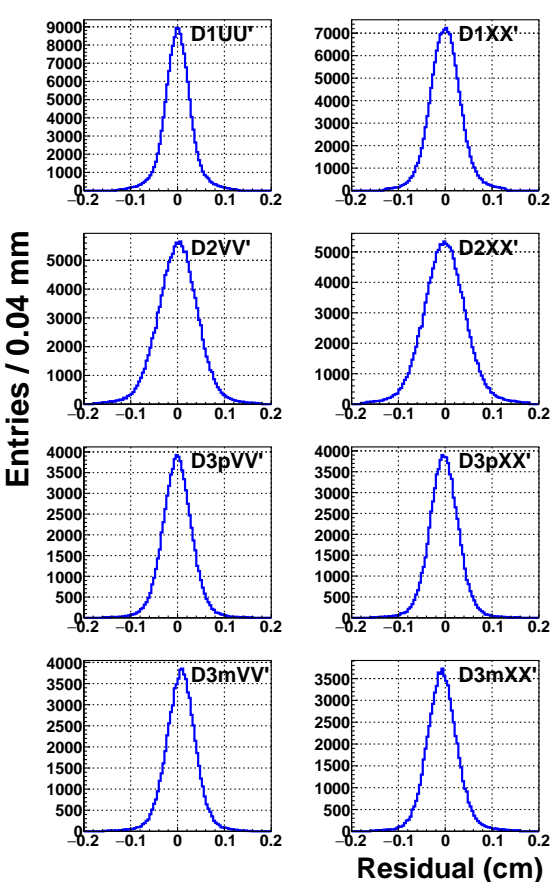

(a) Tracked with all the planes.
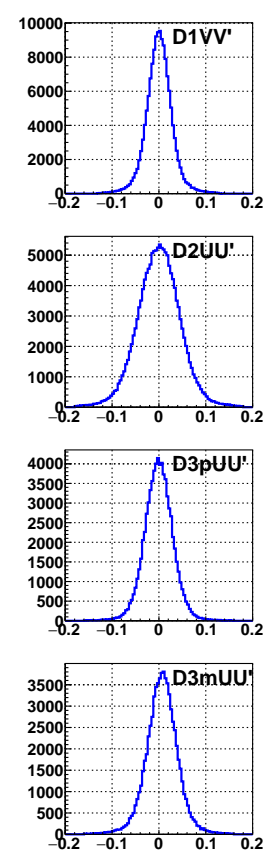
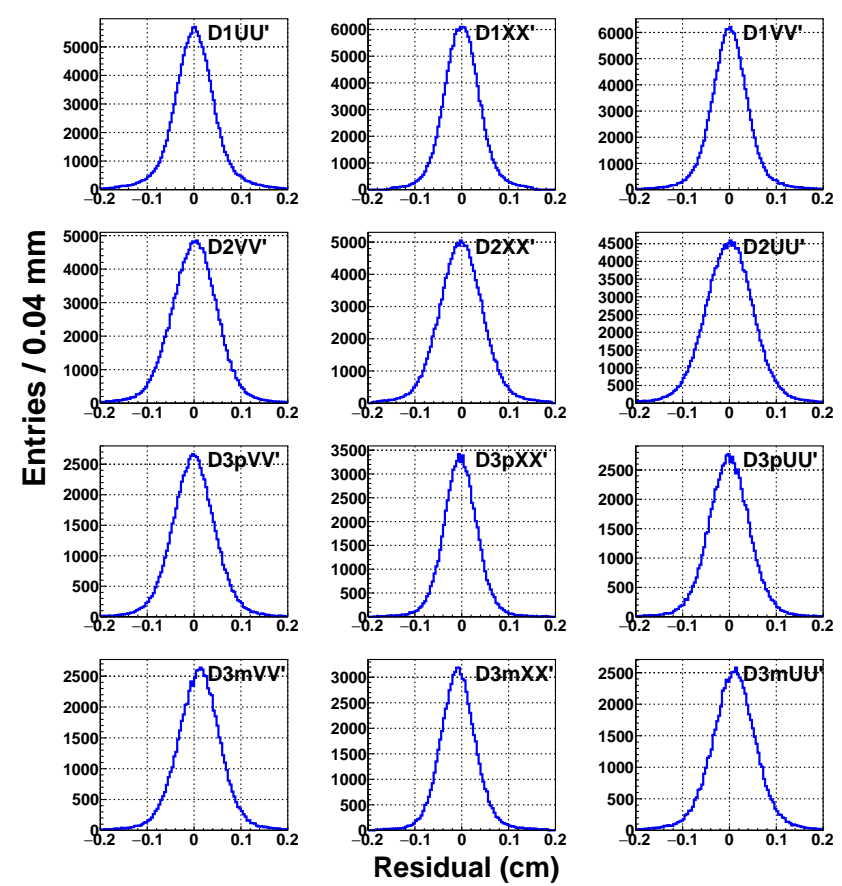

(b) Tracked without the target plane.

Figure 3.21: Residual at each plane. The results using the tracks reconstructed with the target plane are shown in (a). The results using the tracks reconstructed without the target plane are shown in (b).

structed with 18 chamber planes, the other is with the tracks reconstructed without the target plane: for example, with the tracks reconstructed without D1X when the resolution of D1X is evaluated. In the former method, the residual is smaller than the plane position resolution because the hit position on the target plane attracts the reconstructed track and thus affect the result. In the latter method, the residual is larger than the plane position resolution because the residual includes not only the residual on the target plane but also the tracking uncertainty arising from other planes. The effects are mostly cancelled out by taking a geometric mean of the residual evaluated with the target plane and that 
without the target plane [34]:

$$
\sigma=\sqrt{\sigma_{\text {all }} \cdot \sigma_{\text {ignore }}}
$$

where $\sigma$ is the plane position resolution, $\sigma_{\text {all }}$ is the residual evaluated using the tracks with all the planes, and $\sigma_{\text {ignore }}$ is the residual evaluated using the tracks without the target plane.

The resolutions evaluated based on Fig. 3.21 are summarized in Tab. 3.7. Most of them satisfy

Table 3.7: Residual and plane position resolution. The plane resolution of each plane is a geometric mean of standard deviations of residual with and without the target plane.

\begin{tabular}{l|ccc}
\hline Chamber & $\begin{array}{c}\text { Standard Deviation of Residual } \\
(\text { w/ all the planes }) \\
(\mu \mathrm{m})\end{array}$ & $\begin{array}{c}\text { Standard Deviation of Residual } \\
(\text { w/o target plane })\end{array}$ & $\begin{array}{c}\text { Plane Position } \\
\text { Resolution } \\
(\mu \mathrm{m})\end{array}$ \\
\hline D1UU' & 253 & 382 & 310 \\
D1XX' & 304 & 356 & 328 \\
D1VV' & 241 & 355 & 292 \\
D2VV' & 399 & 463 & 429 \\
D2XX' & 416 & 441 & 428 \\
D2UU' & 420 & 491 & 454 \\
D3pVV' & 296 & 426 & 355 \\
D3pXX' & 292 & 336 & 313 \\
D3pUU & 279 & 414 & 339 \\
D3mVV' & 297 & 429 & 356 \\
D3mXX' & 307 & 354 & 329 \\
D3mUU' $^{\prime}$ & 296 & 433 & 358 \\
\hline
\end{tabular}

the requirement which is $400 \mu \mathrm{m}$. The resolutions on St. 2 chamber planes are larger than the requirement. The requirement is determined based on the resolution of the mass of the muon pairs. The dominant source of mass resolution is not the plane position resolution, but the multiple scattering at FMag (beam dump). Therefore, the requirement of the plane position resolution is not strict. These excesses are tolerable. 


\subsubsection{Proportional Tubes}

St. 4 is used as the muon identification. St. 4 is located downstream of a 1 m thick iron wall.

Figure 3.22 shows the top view and the side view of the proportional tubes. The tracking detector of St. 4 consists of four proportional planes. Each plane consists of a layer and a sub-layer shifted by half a cell size. Each layer consists of nine proportional tube modules. One proportional tube module has eight proportional tubes.

Figure 3.23 shows the structure of the proportional tube. Table 3.8 shows the specification of the proportional tubes. $\mathrm{P} 4 \mathrm{H}(\mathrm{P} 4 \mathrm{~V})$ planes are oriented to measure the horizontal (vertical) direction. A

Table 3.8: Specification of proportional tubes.

\begin{tabular}{ccccccccc}
\hline Detector & $\begin{array}{c}\text { Radius } \\
(\mathrm{cm})\end{array}$ & \# of Layers & $\begin{array}{c}\text { \# of Modules } \\
\text { per Layer }\end{array}$ & $\begin{array}{c}\text { \# of Tubes } \\
\text { per Module }\end{array}$ & $\begin{array}{c}\text { Tilt Angle } \\
(\mathrm{rad})\end{array}$ & $\begin{array}{c}x \\
(\mathrm{~cm})\end{array}$ & $\begin{array}{c}y \\
(\mathrm{~cm})\end{array}$ & $\begin{array}{c}z \text {-position } \\
(\mathrm{cm})\end{array}$ \\
\hline P1H & 5.08 & 2 & 9 & 8 & 0 & 368.3 & 388.6 & 2102.1 \\
P1V & 5.08 & 2 & 9 & 8 & $\pi$ & 388.6 & 368.3 & 2178.8 \\
P2H & 5.08 & 2 & 9 & 8 & 0 & 368.3 & 388.6 & 2394.4 \\
P2V & 5.08 & 2 & 9 & 8 & $\pi$ & 388.6 & 368.3 & 2371.3 \\
\hline
\end{tabular}

proportional tube consists of a $5.08 \mathrm{~cm}$ wide aluminum tube with wall thickness of $0.16 \mathrm{~cm}$ and a gold-plated $20 \mu \mathrm{m}$ tungsten anode wire. The gas mixture is the same as the drift chambers. The tubes are grounded and $+1800 \mathrm{~V}$ is applied to anode wires.

\subsubsection{Readout System}

The readout system consists of three parts: ASDQ card, Level Shifter Board, and TDC card. The drawing of the readout system is shown in Fig. 3.24.

\section{ASDQ card}

ASDQ stands for Amplifier-Shaper-Discriminator-Charge Integrator (Q). Figure 3.25 shows the ASDQ card used in SeaQuest. The ASDQ card is designed at the University of Pennsylvania for CDF experiment [35]. All the features are included into the ASDQ chip at the center of the ASDQ card. It has eight channel input connected to the sense wires of the drift chambers. About 800 ASDQ cards in total are used for the SeaQuest.

A signal from the sense wires of drift chambers is amplified by the ASDQ card. The ASDQ card discriminates the amplified signals with the threshold set by the Level Shifter Board which is explained in the next paragraph.

\section{Level Shifter Board}

The role of the Level Shifter Board is to convert the ASDQ signals to standard LVDS signal. Figure 3.26 shows the Level Shifter Board. It has eight input slots for the cables which are connected to the ASDQ cards. It also has eight output slots. Therefore, it can treat 64 channels of the drift chamber wires. 


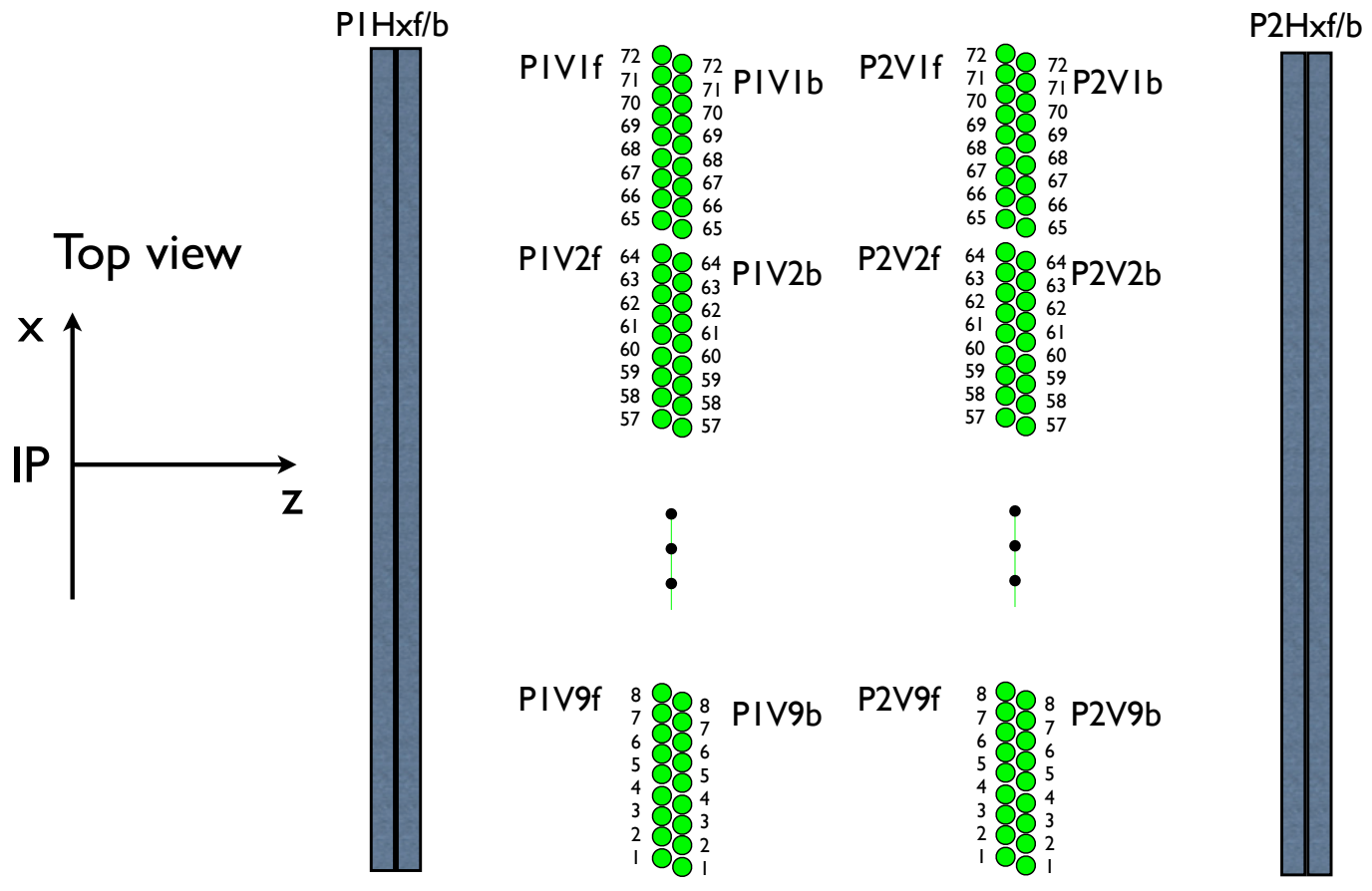

(a) Top view

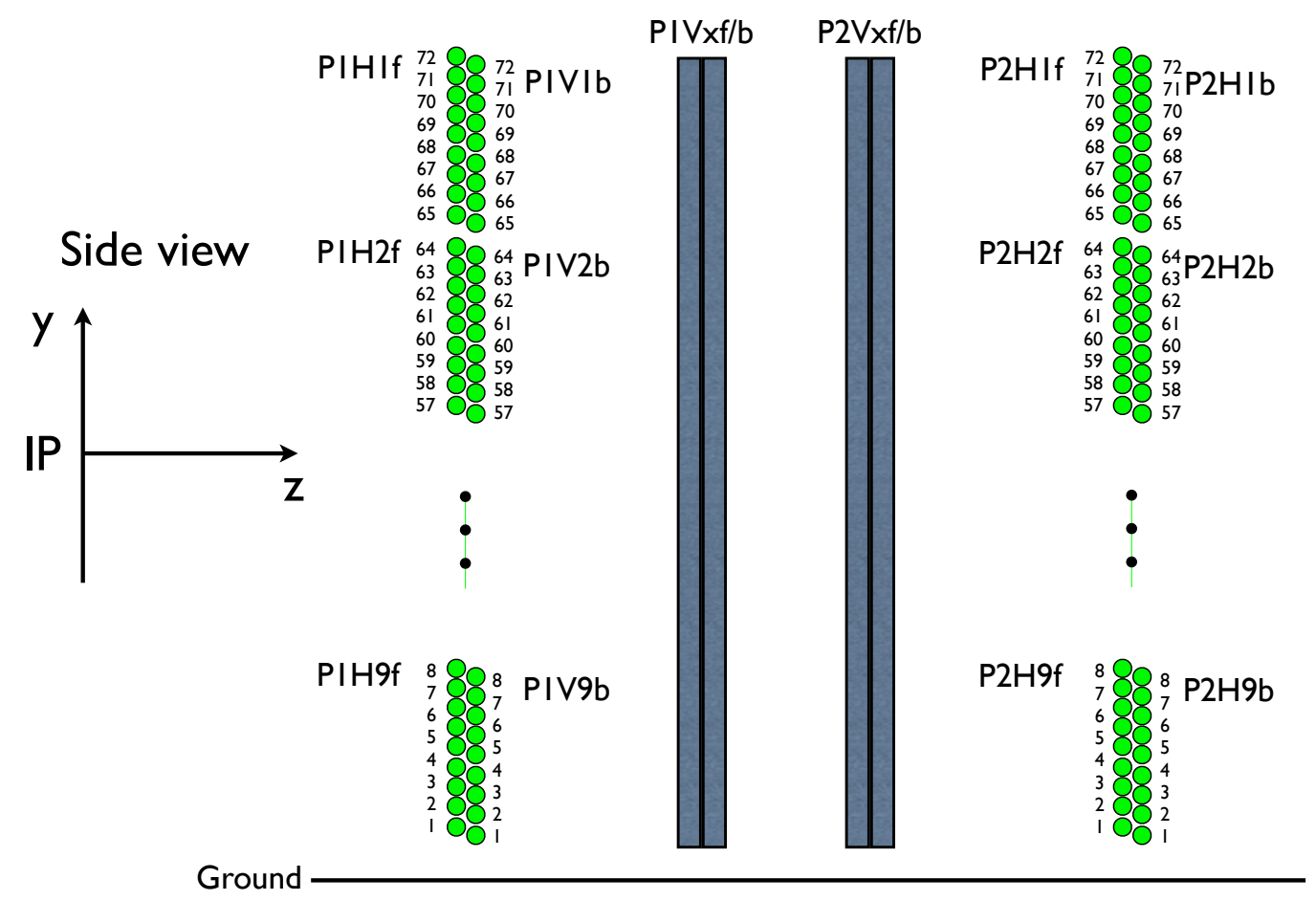

(b) Side view

Figure 3.22: Top view and side view of the proportional tubes. 


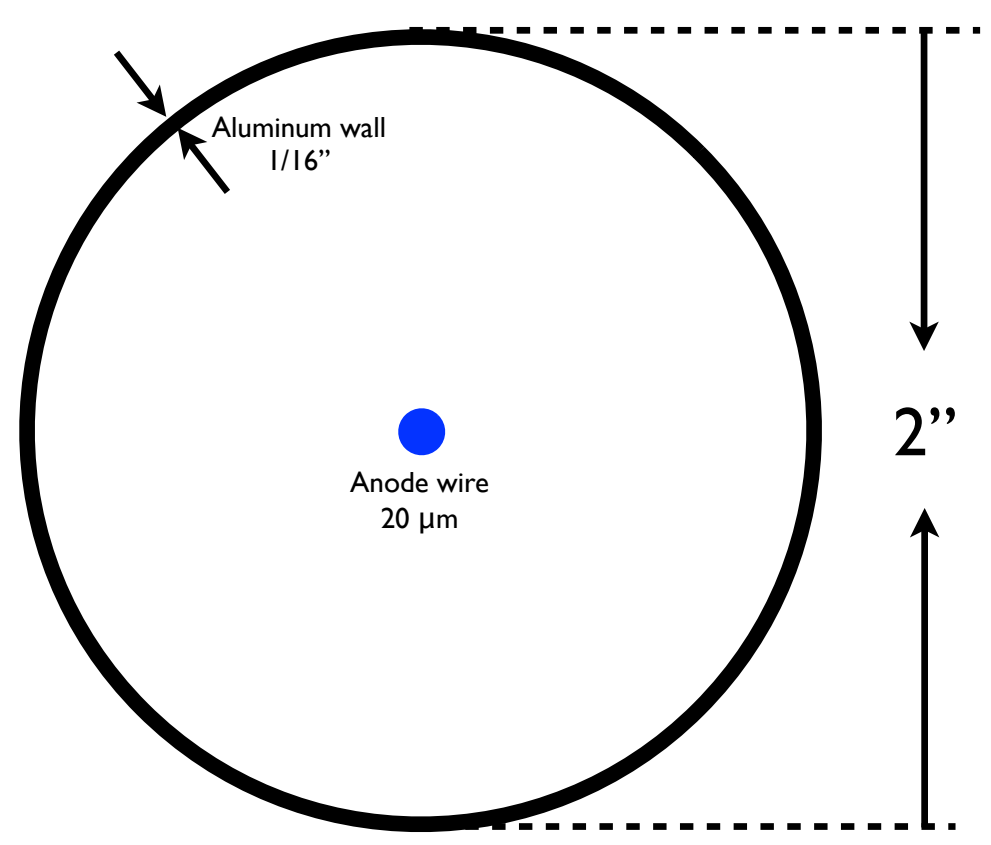

Figure 3.23: Structure of the proportional tubes.

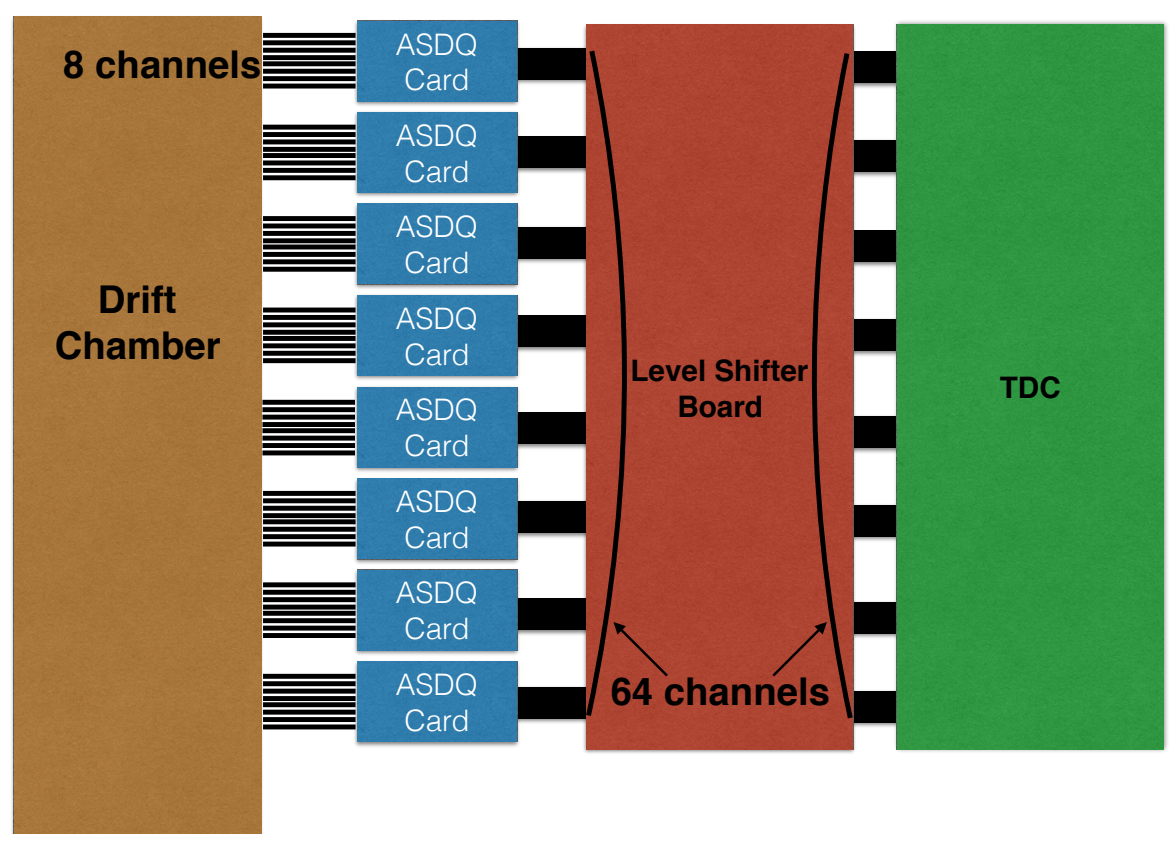

Figure 3.24: Drawing of the readout system. The signals from drift chambers are sent to the ASDQ cards. The output signals from ASDQ cards are converted to a LVDS signals by Level Shifter Boards, and then read by the TDC cards. 


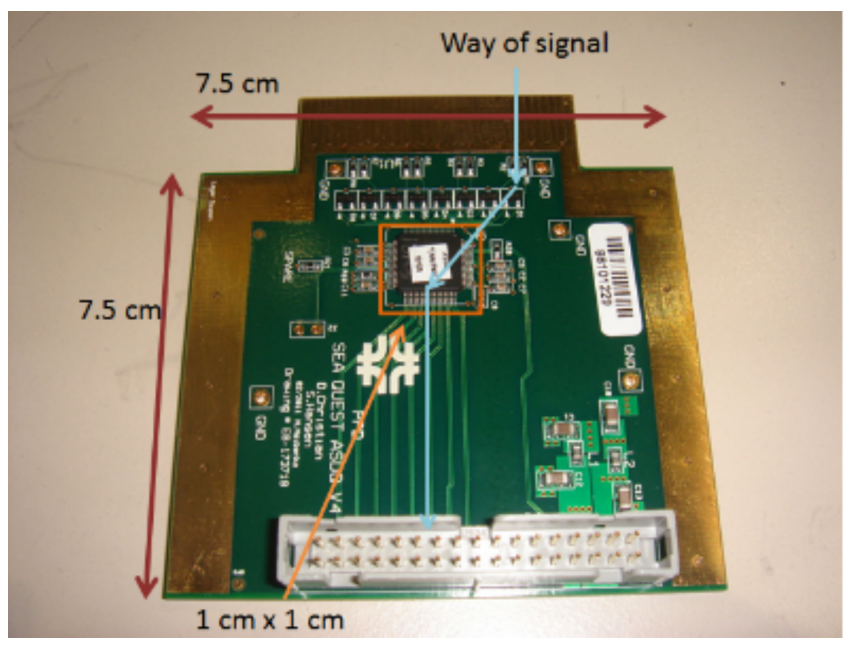

Figure 3.25: ASDQ card. It is $7.5 \mathrm{~cm} \times 7.5 \mathrm{~cm}$. An ASDQ chip is placed at the center of the card. The top side is connected to the wires, and the signals processed by the ASDQ chip are read out from bottom side.

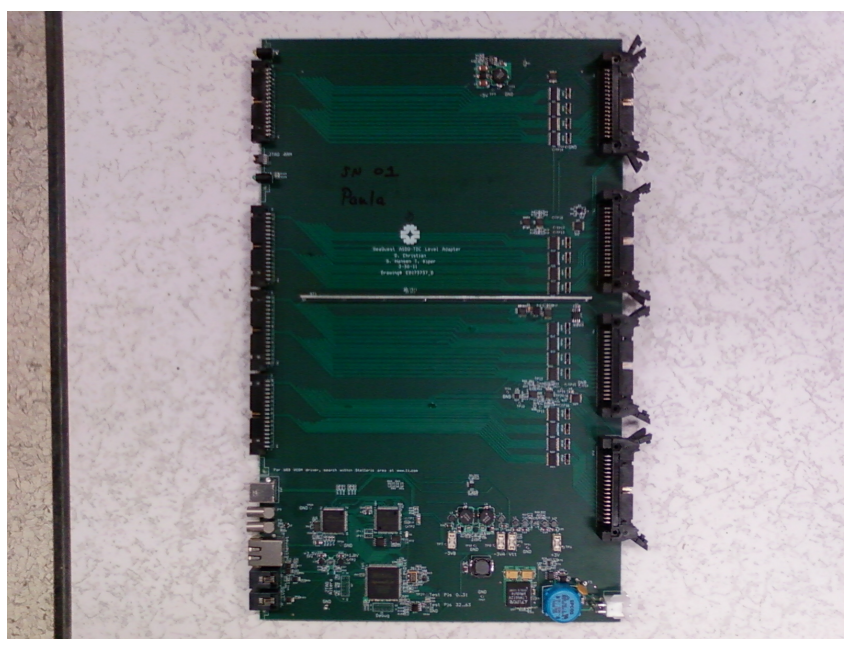

Figure 3.26: Level Shifter Board. The Level Shifter Board receives the signals from ASDQ cards from the right side of the picture. After processing, the Level Shifter Board sends LVDS signals from the left side. 
In addition to the signal converting, the Level Shifter Board supplies the ASDQ card a low voltage and controls the thresholds of the ASDQ cards.

\section{TDC card}

TDC stands for "time-to-digital converter". It converts the time information to the digital signal. SeaQuest uses the TDCs which have 6U VMEbus form factor and are equipped with a low-power and radiation-hardened Microsemi ProASIC2 Flash based FPGA [36]. The TDC has eight input slots connected to the Level Shifter Boards. One input slot corresponds to eight channels, therefore it has 64 channels in total.

The role of TDC is to measure the difference between the trigger time and the hit time on the drift chambers. This information on the drift time is then converted to the drift distance, and the track reconstruction is performed. The TDC receives two types of signals: detector signals and trigger signals. The trigger signals are used as common "stop" signals, and the detector signals are used as "start" signals.

\subsection{Trigger}

The trigger system aims to select the events with opposite-sign muon pairs originating from the DrellYan process or charmonium state of $J / \psi$. The system uses the discriminated hodoscope signals. The trigger system concentrates on measuring the high mass $(4-10 \mathrm{GeV})$ muon pairs since the SeaQuest experiment aims to measure $\bar{d} / \bar{u}$ at high $x_{2}$.

The trigger system holds 10 different types of triggers. The triggers are classified into two types: FPGA-based trigger and NIM-based trigger. Each of FPGA-based trigger and NIM-based trigger has five triggers. Seven triggers out of the 10 triggers are used in the normal data taking: FPGA-1, -2, -3 , $-4,-5$ and NIM-1 and -3. The FPGA-based trigger logic electronics are based on the CAEN v1495 VME bus modules [36] including the Field-Programable Gate Array (FPGA). The NIM-based trigger logic electronics are based on the NIM modules.

The trigger names and their characteristics are summarized in Tab. 3.9. In the table, "T" ("B")

Table 3.9: Name and characteristics of each trigger.

\begin{tabular}{lcccr}
\hline Trigger Name & Target & Hodoscope side & $P_{t}$ cut & Prescale factor \\
\hline FPGA-1 & $\mu^{+} \mu^{-}$ & TB or BT & - & 1 \\
FPGA-2 & $\mu^{+} \mu^{-}$ & TT or BB & - & 10000 \\
FPGA-3 & $\mu^{+} \mu^{+}$or $\mu^{-} \mu^{-}$ & TB or BT & - & 123 \\
FPGA-4 & $\mu^{+}$or $\mu^{-}$ & T or B & - & 25461 \\
FPGA-5 & $\mu^{+}$or $\mu^{-}$ & T or B & $>3 \mathrm{GeV}$ & 2427 \\
\hline NIM-1 & $\mu^{+}$or $\mu^{-}$ & T or B & - & 31991 \\
NIM-3 & - & - & - & 125 \\
\hline
\end{tabular}

stands for "Top" ("Bottom") indicating the location of the hodoscope. Prescale factor is the rate to 
take the events. For example, if there are 100,000 events which satisfy the FPGA-2 trigger, 10 events out of the 100,000 events are triggered because the prescale factor is 10,000. The prescale of FPGA-1 trigger is set to be 1 since the FPGA-1 trigger events are used in the physics data analysis.

\subsubsection{NIM-based Trigger}

NIM-1 trigger requires a coincidence signal of $(\mathrm{H} 1 \mathrm{~T} \cap \mathrm{H} 2 \mathrm{~T} \cap \mathrm{H} 3 \mathrm{~T} \cap \mathrm{H} 4 \mathrm{~T})$ or $(\mathrm{H} 1 \mathrm{~B} \cap \mathrm{H} 2 \mathrm{~B} \cap \mathrm{H} 3 \mathrm{~B} \cap \mathrm{H} 4 \mathrm{~B})$. This trigger was used before the FPGA-based trigger systems become ready. It was also used for the detector commissioning.

NIM-3 trigger is a random trigger. It requires a coincidence of a RF-signal provided by the Fermilab accelerator division and $7.5 \mathrm{kHz}$ regular pulse signal produced by the gate generator. This trigger doesn't have any biases while the others depends on their trigger logic. Therefore, the events taken with this trigger are able to be used for the background study. For example, the mixed dataset of Monte-Carlo simulation events and NIM3 triggered events is useful. An event including a Drell-Yan muon pair has a muon pair and also background noises. Some background noises are related to the true muon tracks, but others are not. NIM-3 events reproduce the background noises uncorrelated with tracks.

\subsubsection{FPGA-based Trigger}

The main background is accidental coincidence of the single muons originating from decays of mesons $(\pi, \mathrm{K}, \mathrm{D}$, etc.). It is important to separate the Drell-Yan and $J / \psi$ signals from the background for the efficient measurement. The SeaQuest experiment uses the FPGA-based trigger system to reduce those background signals [37].

The trigger system consists of

- four hodoscope arrays (St. 1, 2, ,3, 4),

- Level-1 track finder, and

- Level-2 track correlator.

Four hodoscope arrays are explained in subsection 3.3.3.1. There are two types of hodoscope arrays: X-plane and Y-plane. The SeaQuest trigger system uses the hit pattern of the hodoscope hits. The hits of the X-plane hodoscope arrays construct a bent line according to the muon momentum. The hits of the Y-plane hodoscope arrays construct a straight line. The hit pattern of the X-plane hodoscope arrays is used for the Level-1 track finder.

The Level-1 track finder has a look-up-table which contains predicted hit patterns of $\mathrm{H} 1, \mathrm{H} 2$, $\mathrm{H} 3$ and H4 hits. A hit pattern corresponds to muon tracks coming from the target position. The hit patterns are predicted with the Monte-Carlo simulation of Drell-Yan and $J / \psi$. Only the hit patterns of high mass muon pairs are included in the Level-1 track finder because the SeaQuest aims to measure high mass Drell-Yan events. Therefore, a set of hit patterns used in Level-1 track finder determines the acceptance of kinematics. A hit pattern used for the trigger is called a "trigger-road". A set of trigger-roads is called a "Roadset". Roadsets are optimized many times to detect desired muon pairs 


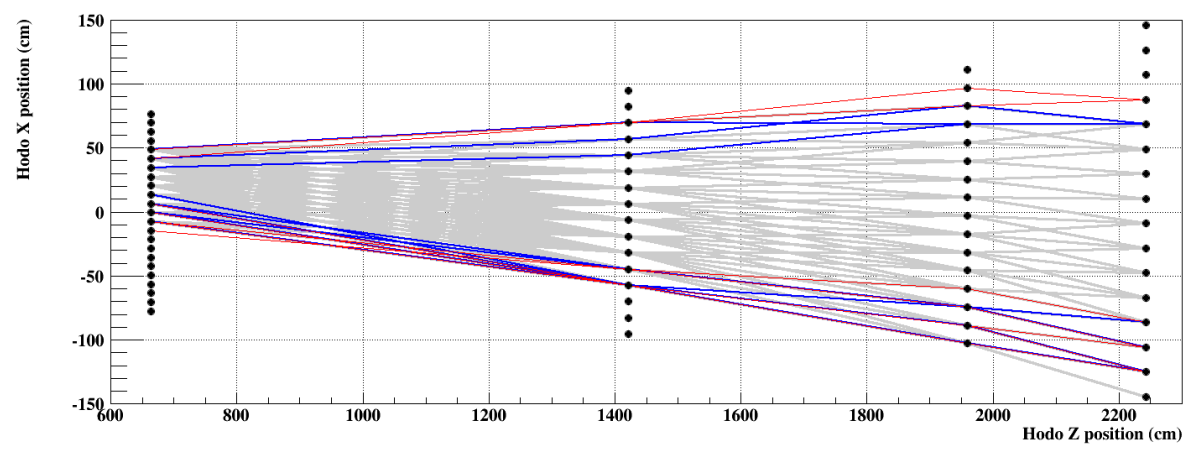

Figure 3.27: Visualization of the fired trigger-roads of (Top, + ). The data are a part of Roadset 70. The red lines are the hottest 10 trigger-roads, and blue lines are the next 10 hottest trigger-roads. The other lines are the other trigger-roads in Roadset 70.

effectively. The version of the roadset is arranged with suffix number, for example, "Roadset 57". The name of a data set is the same as the name of the version of the roadset used for the data taking. The roadset is common to all the FPGA-based triggers. The trigger-roads are classified into four types based on the hodoscope location (top or bottom) and the charge of the track: (Top, +), (Top, - ), (Bottom, +), (Bottom, - ).

The Level-2 track correlator receives the results of Level-1 track finder. The Level-2 track correlator works differently among the triggers. As shown in Tab. 3.9, for example, the FPGA-1 trigger requires muon pairs of $(\mathrm{Top},+) \&($ Bottom, -$)$ or $($ Bottom, +$) \&($ Top, - ). The trigger of each FPGA-1, -2, -3, and -4 fires if the Level-2 track correlator receives the Level-1 results which includes the predetermined trigger-road (combination). The FPGA-5 trigger requires an additional requirement of $P_{t}>3 \mathrm{GeV}$. The average $P_{t}$ of each trigger-road is pre-calculated. If a hit pattern is included in the roadset and the average $P_{t}>3 \mathrm{GeV}$, the FPGA-5 trigger fires.

The FPGA-1 and -2 triggers are unlike-sign dimuon triggers. They require at least one $\mu^{+}$triggerroad and $\mu^{-}$trigger-road. The FPGA-1 trigger requires that one of the trigger-roads is a top triggerroad and another is a bottom trigger-road. This kind of trigger-road combination corresponds to the dimuon originating from Drell-Yan process or $J / \psi$. The events taken with FPGA-1 trigger are used for the data analysis. Therefore, the prescale is set to be 1 . The FPGA-2 trigger requires that both of the $\mu^{+}$and $\mu^{-}$fire the same hodoscope side: top \& top or bottom \& bottom.

The FPGA-3 trigger is a like-sign dimuon trigger. It requires that at least two same sign triggerroads fire. It is used for the random background estimation.

The FPGA-4 and FPGA-5 are single muon triggers. The FPGA-4 trigger accepts all the single trigger-roads while FPGA-5 accepts the trigger-roads with average $P_{t}>3 \mathrm{GeV}$. The FPGA-4 trigger is suitable to investigate the feature of the detectors since it covers the whole region of the detectors. The advantage of FPGA-5 trigger is that it fires only high $P_{t}$ trigger-roads. The FPGA-5 triggered events are suitable for investigating the single muons which can be used in the physics analysis.

Figure 3.27 shows the visualization of the fired trigger-roads of (Top, + ). The data are a part of the Roadset 70 . The 10 hottest trigger-roads are shown with red lines, and the next 10 are shown with blue lines. 
The datasets used in this thesis are Roadsets 57, 62, 67, and 70. The Roadset 57 is the first successful roadset for the physics data acquisition. The Roadset 62 has more trigger-roads than the Roadset 57. Most of them are the same, but some trigger-roads for the dark-photon search are added. Some trigger-roads in the Roadset 62 are hot-roads. A hot-road is defined as a high rate trigger-road due to the noisy hodoscopes. The probability is high that an event triggered with a hot-road has a fake muon. In order not to measure fake muons, the Roadset 67 was created with the Roadset 62 trigger-roads which are not hot-roads. There were some additional hot-roads in Roadset 67 . They were removed and then the Roadset 70 was created. 


\subsection{Data Acquisition System}

The data acquisition system is divided into three parts: Event DAQ, Scaler DAQ, and Beam DAQ. The Event DAQ records the information of main detectors such as drift chambers, hodoscopes, proptubes, and so on. The Scaler DAQ records the scaler information on a $7.5 \mathrm{kHz}$ clock. The Beam DAQ records the information provided by the Cherenkov counter information.

\subsubsection{Event DAQ}

The Event DAQ is the main DAQ which reads from detectors event-by-event. The Event DAQ is a VME-based "CODA" (CEBAF On-line Data Acquisition) system developed by Thomas Jefferson National Accelerator Facility (JLab) [38]. A chain of VME crates is shown in Fig. 3.28.



Figure 3.28: A chain of VME crates. There are 14 VME crates in total.

The Event DAQ consists of $14 \mathrm{VME}$ crates. Each of $13 \mathrm{VME}$ crates out of 14 is composed of VME processor, Trigger Interface (TI) and TDCs. The VME processor is called "ROC" (Readout Controller). It reads out the events in the TDCs. Each TDC digitizes the signals from the detectors. The TI manages the readout. The other VME crate is called "Trigger Supervisor (TS)". It retains 12 input trigger slots. The first four trigger inputs can be prescaled by up to 24 bits and are used for four FPGA-based triggers of FPGA-1, $-2,-3$ and -4 triggers. The next four trigger inputs can be prescaled up to 16 bits and are used for a FPGA-based trigger of FPGA-5 trigger and three NIM-based triggers 
of NIM-1, -2 and -3 triggers. The remaining four trigger inputs are not prescalable. They are used for two NIM-based triggers of NIM-4 and -5 triggers and "BOS" (beginning of spill) and "EOS" (end of spill) signals. The BOS and EOS signals are provided by the Fermilab Accelerator Division.

The role of the TS is to control the other $13 \mathrm{VME}$ crates. The TS receives the trigger signals of FPGA-based triggers, NIM-based triggers or the both. The trigger signals have already passed the QIE veto logic processed in a NIM module. The QIE veto logic ensures that the beam intensities of the RF-buckets around the triggered RF-bucket are not too high. When the beam intensity of each of the triggered RF-bucket eight RF-buckets before/after the triggered RF-bucket is higher than the inhibit threshold, the trigger is rejected and is not sent to the TS. The TS transmits the received trigger signals to the TIs of the other $13 \mathrm{VME}$ crates and the QIE board. Once the TI receives the trigger signals from TS, the TI acknowledges the ROC to readout the events. The TI acknowledges back to TS when the ROC finishes reading out the events from TDCs. The QIE board reads out the beam intensities of the 16 RF-buckets before the triggered RF-buckets, RF-buckets, and 16 RF-buckets after the triggered RF-buckets. After receiving signals from all the ROCs that the readout is completed, the TS is ready for the next trigger again.

The TS doesn't receive any triggers during the readout. The readout time corresponds to the event dead time. The average dead time is $\sim 150 \mu$ s. The following are the breakdown of the dead time:

- the interrupt itself: $10 \mu \mathrm{s}$

- copy-in-progress time of TDC: $32 \mu \mathrm{s}$

- TDC read out: $300 \mathrm{~ns}$ per 1 hit (32 bit)

\subsubsection{Scaler DAQ}

The Scaler DAQ monitors the spectrometer, trigger, and beam condition on spill-by-spill. It works independently of the Event DAQ: whether the Event DAQ works or not. As with the Event DAQ, the Scaler DAQ is driven by the CODA. The Scaler DAQ system consists of one VME crate with four VME scaler modules.

One is used for measuring the duty factor of the beam in $7.5 \mathrm{kHz}$ scale. Its trigger is the coincidence of the $7.5 \mathrm{kHz}$ gate generator and the RF-signal. The VME scaler counts the number of the hodoscope hits and calculates the duty factor.

The other three VME scalers record some kinds of rates in the spill-level. They are triggered by BOS or EOS signals. They collect the data of

- the count of the trigger fired in Event DAQ,

- the intensity of the beam in a spill, and

- the hit rate of the hodoscope arrays.

The data are analyzed by the independent program to monitor the condition of detectors and triggers during the data acquisition runs. The results of the analysis are displayed on a web page so that the shift personnel can monitor them. 


\subsubsection{Beam DAQ}

The Beam DAQ is driven on the QIE board. The Beam DAQ reads out the data measured by the Cherenkov counter. It records the $53 \mathrm{MHz}$ beam structure. The Beam DAQ collects

- the beam intensity of each RF-bucket,

- the beam intensity of each of inhibited RF-buckets,

- the beam intensity of missed RF-buckets during the readout dead time, and

- the sum of beam intensity $I$ and sum of the square of beam intensity $I$ in a spill.

The fourth item is used for calculating the duty factor of the beam in the $53 \mathrm{MHz}$ scale. The duty factor is defined as follows:

$$
D=\frac{\langle I\rangle^{2}}{\left\langle I^{2}\right\rangle}
$$

which indicates the stability of the beam. 


\subsection{Monte Carlo Simulation}

The SeaQuest experiment uses two types of Monte-Carlo simulation: Fast Monte Carlo (FastMC) and Geant4-based Monte Carlo (GMC).

The FastMC was used for the E866 experiment, which is the previous Drell-Yan experiment performed at Fermilab. It is also used in the SeaQuest proposal.

The GMC is mainly used for the analysis in the SeaQuest experiment. It is based on the Geant4 simulation package [39] developed by CERN. The advantage of the GMC is that the effect of the magnetic field and detector responses and so on can be taken into account. The procedure of generating the GMC is the following:

1. Generate dimuons randomly in the field of invariant mass of $2<M<10 \mathrm{GeV}$ and Feynman $x$ of $-1<x_{F}<1$.

2. Apply the quality cuts.

- $P_{T}^{2}>0$

- $0<x_{1}<1$

- $0<x_{2}<1$

$P_{T}$ is the transverse momentum of the virtual photon.

3. Add weight to each event.

- The weight is determined based on the cross section of each process.

- Drell-Yan process: Eq. (2.11), only up, down, charm and strange flavors are included.

- $J / \psi$ and $\psi^{\prime}$ production: the cross section is taken from [40].

- The PDF parameterizations are taken from CTEQ6 [41].

4. Apply the acceptance cuts.

- The two muon tracks should pass the region covered with each station's hodoscope array and drift chamber.

There are two types of GMC: " $4 \pi$ " and "accepted". The " $4 \pi$ " production is just before fourth step. The "accepted" production is after the fourth step. The accepted production is usually used for the analysis, but the $4 \pi$ production is used for the acceptance study.

Figure 3.29 shows the acceptance of " $4 \pi$ " GMC (black points) and "accepted" GMC (red points). The "accepted" GMC events are concentrated at high $x_{1}$ and low $x_{2}$ due to the SeaQuest spectrometer acceptance. 




Figure 3.29: Acceptance of " $4 \pi$ " GMC and "accepted" GMC. Black points show $4 \pi$ GMC and red points show accepted GMC. Red points are concentrated at right bottom of the figure. 


\section{Chapter 4}

\section{Data Analysis}

In this chapter, dimuon reconstruction, selection of dimuons, cross section ratio of the proton and deuteron, and corrections are described.

\subsection{Dataset}

The data used in this thesis are taken in 2014 and 2015. The datasets are classified with Roadset as shown in section 3.4. The datasets used in this thesis are Roadset 57, 62, 67, and 70.

\subsection{Dimuon Reconstruction}

Events are analyzed using a track reconstruction tool. The track reconstruction procedure consists of three steps: pre-tracking analysis, single track reconstruction, and reaction vertex reconstruction as described below in detail. Figure 4.1 shows the procedure of the dimuon reconstruction.

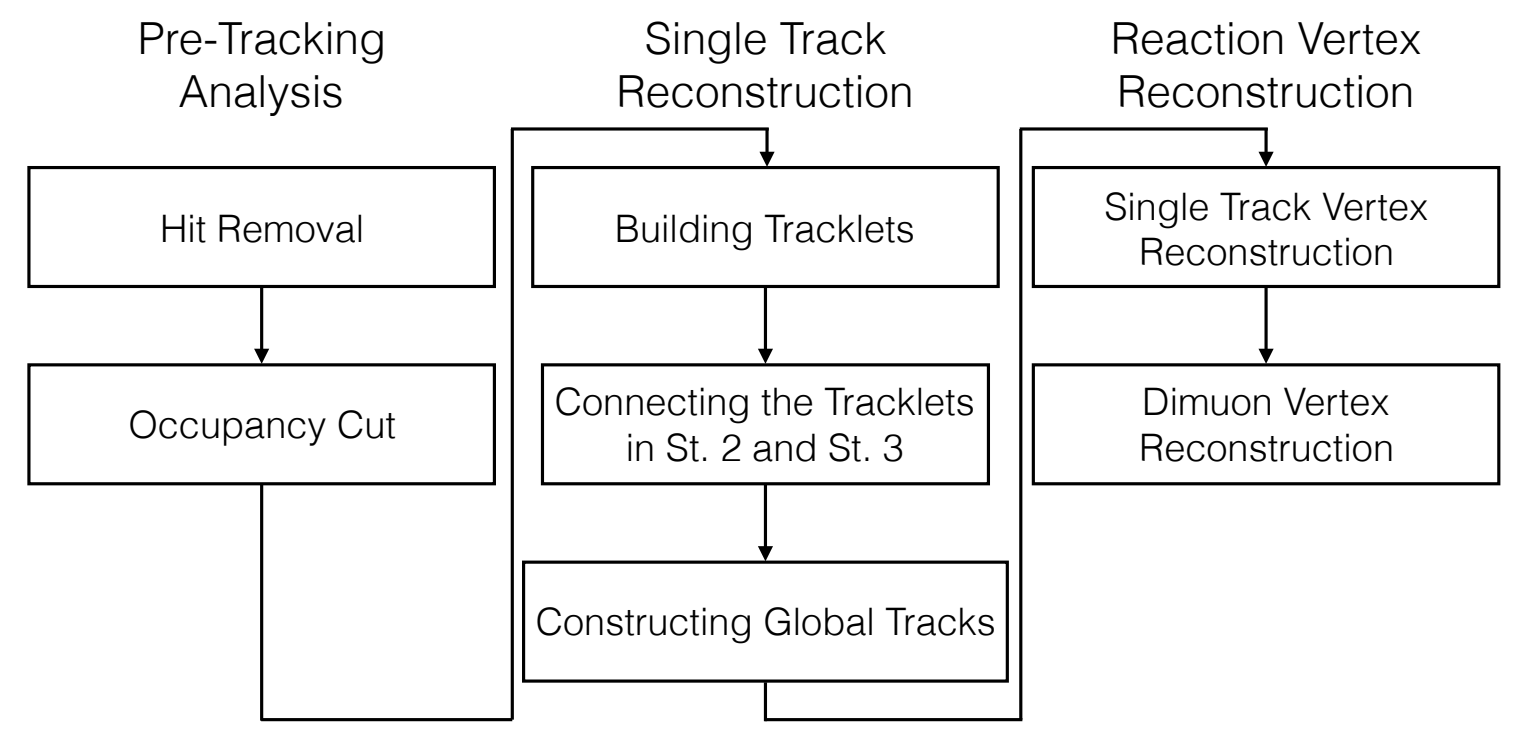

Figure 4.1: Procedure of the dimuon reconstruction. The dimuon reconstruction procedure is divided into three parts: pre-tracking analysis, single track reconstruction and vertex reconstruction. 


\subsubsection{Pre-tracking Analysis}

Not all the events can be reconstructed because some events have too many chamber hits and the tracking may fail. The purposes of the pre-tracking analysis are reduction of chamber hits and rejection of un-analyzable events. The pre-tracking analysis is divided into two steps: hit removal and occupancy cut.

\subsubsection{Hit Removal}

There are many chamber hits which are not correlated with the muon track in an event. The processing time of the track reconstruction is delayed by such kind of hits. Those hits are called extra hits. Removing the extra hits is very helpful for the track reconstruction [42].

The extra hits are classified into four types: out-of-time hits, after-pulses, cluster hits and random noises.

Out-of-time hits are not in the range of the TDC time window. They are discarded because they are not proper hits.

Random hits can be removed using hodoscope hit positions. The places of true hits, which are track correlated hits, are always included in the range of the hodoscope paddles. On the other hand, random hits are not correlated with a muon track position. Therefore, random hits are removed by the chamber-hodoscope hit matching.

After-pulses rise at signal wires following the true signal. Only the first hit on a wire is used for the analysis in order to remove after-pulses.

There are extra hits which are contiguous to hit wires. A group of contiguous hits is called a "hit cluster". The types of hit clusters are listed in Tab. 4.1. Here, "size" is the number of hits in one

Table 4.1: Types of hit clusters.

\begin{tabular}{lll}
\hline Size & \multicolumn{1}{c}{ Type } & \multicolumn{1}{c}{ Characteristics } \\
\hline \hline 2 & Edge Hit & $\begin{array}{l}\text { A muon hits the edge of cell and produces a signal on the neighboring } \\
\text { wire. If the drift distance of one of the two hits is larger than 90\% of } \\
\text { cell size and that of the other is larger than 40\%, the cluster is regarded } \\
\text { as "Edge hit". }\end{array}$ \\
& & $\begin{array}{l}\text { The difference of the drift times is less than } 8 \mathrm{~ns} . \text { It appears only on St. } \\
3+\text { drift chamber in Run II data. }\end{array}$ \\
& $\begin{array}{l}\text { Electronic } \\
\text { Noise }\end{array}$ & $\begin{array}{l}\text { Average of the differences of drift times of neighboring wire hits is less } \\
\text { than 10 ns. }\end{array}$ \\
Electronic & Noise & $\begin{array}{l}\text { These clusters are arise from delta rays created by muons. Average of } \\
\text { the differences of drift times of neighboring wires is 10 ns or larger. }\end{array}$ \\
\cline { 2 - 3 } & Delta Ray
\end{tabular}

cluster.

The "Edge hit" clusters appears when a muon passes at the edge of two cells of the drift chambers and hits appear on both cells. One hit in the "Edge hit" cluster can be removed because both of them 


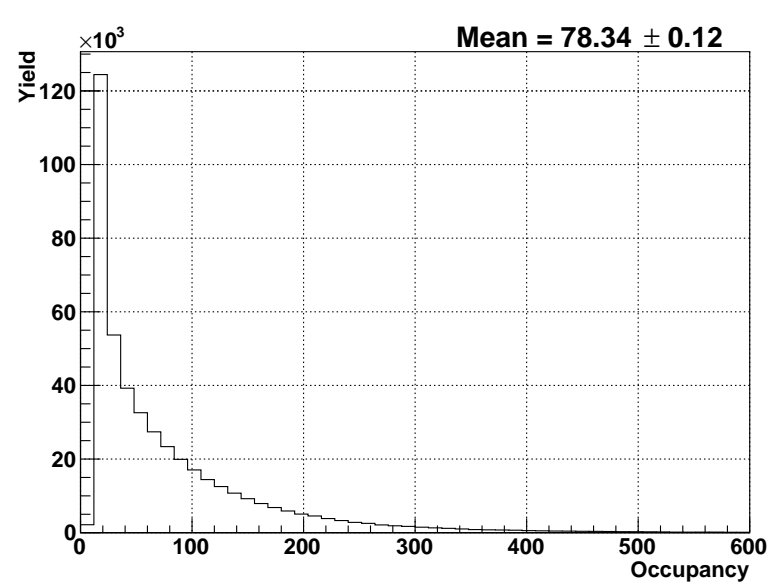

(a) Before hit removal (linear scale).

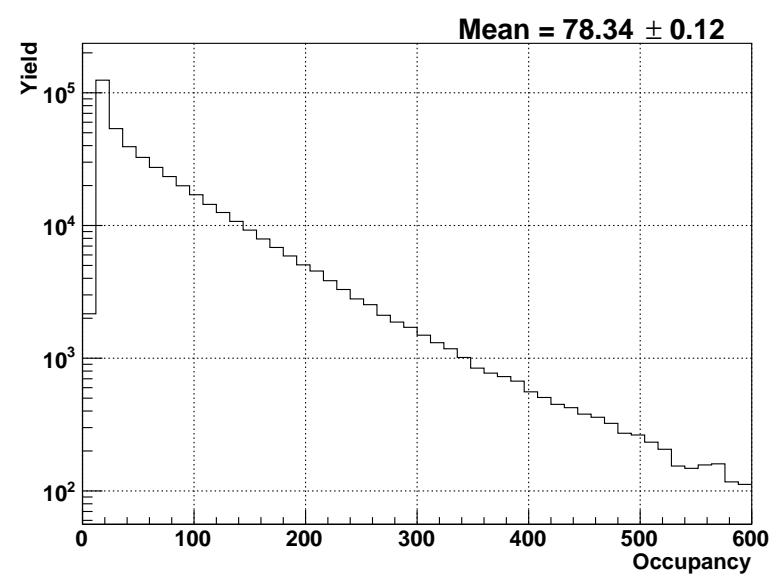

(c) Before hit removal (log scale).

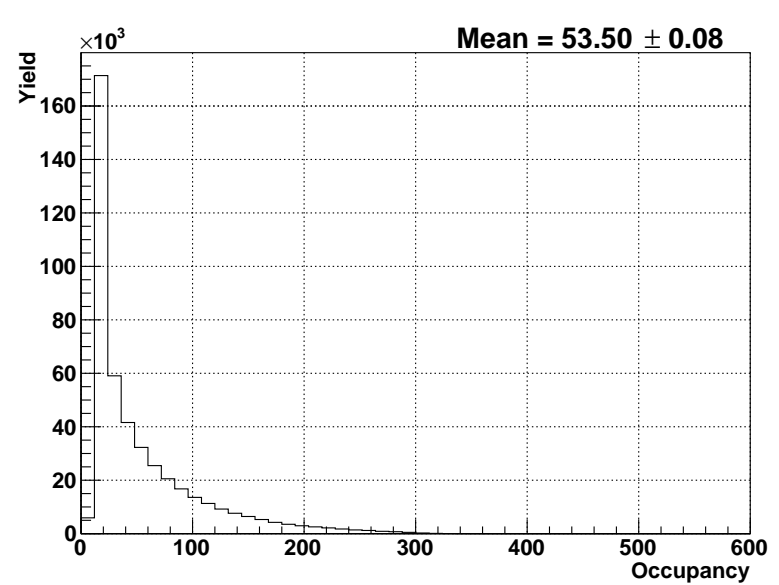

(b) After hit removal (linear scale).

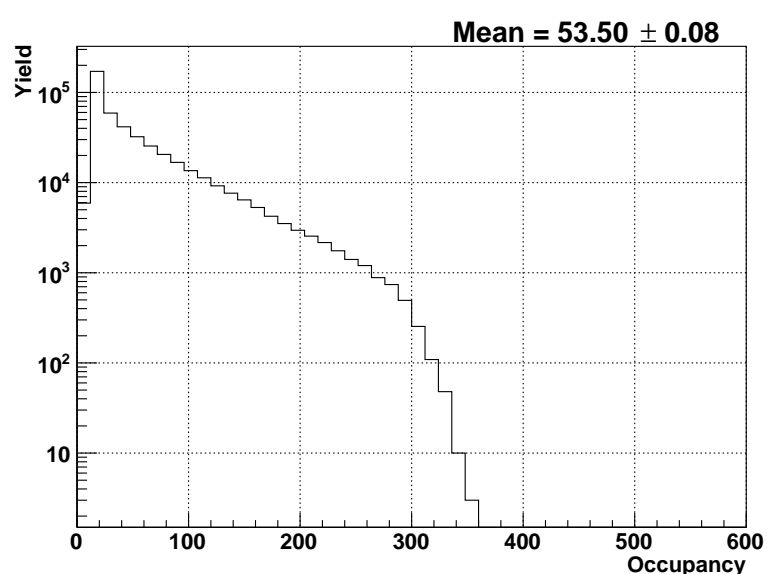

(d) After hit removal (log scale).

Figure 4.2: Number of hits in the St. 1 drift chamber. The distributions before the hit removal are shown in (a) (linear scale) and (c) (log scale). The distributions after the hit removal are shown in (b) (linear scale) and (d) (log scale). The average numbers of hits in St. 1 drift chamber are 78 ((a) and (c)) and 53 ((b) and (d)).

are true hits and only one of them should be used for the track reconstruction. The larger drift distance hit is removed.

When a charged particle passes the drift chamber, the electronic circuit becomes unstable, and thus the probability that the noises pass the threshold becomes higher. "Electronic noise" clusters due to this effect have only track un-correlated hits. All the hits in that cluster are removed as extra hits.

A delta ray is emitted from a charged particle. The "Delta ray" clusters are produced around the true hits. One of the "Delta ray" cluster hits must be a true hit, and the others are extra hits. Therefore, all the hits in the cluster except the hits on both sides of the cluster are removed as extra hits.

Figure 4.2 shows the number of hits in the St. 1 drift chamber before and after the hit removal using random trigger data. After the hit removal, the average number of hits is reduced by about $30 \%$.

Some true hits are removed by the hit removal. The effect is estimated using a part of real data. The following procedure is performed (Fig. 4.3):

- The usual single track reconstruction is performed. Here, the data before the track reconstruc- 
tion are called raw data, and the data after the track reconstruction are called tracked data.

- Find a plane without track associated hits. Here, such kind of plane is called empty plane.

- Compare with the raw data and check whether the raw data have a hit near (within $3 \times$ plane resolution) the track position on the empty plane. If such kind of hit exists, the "hit removal" removes a true hit. The amount of such kind of hits is less than $1.0 \%$.

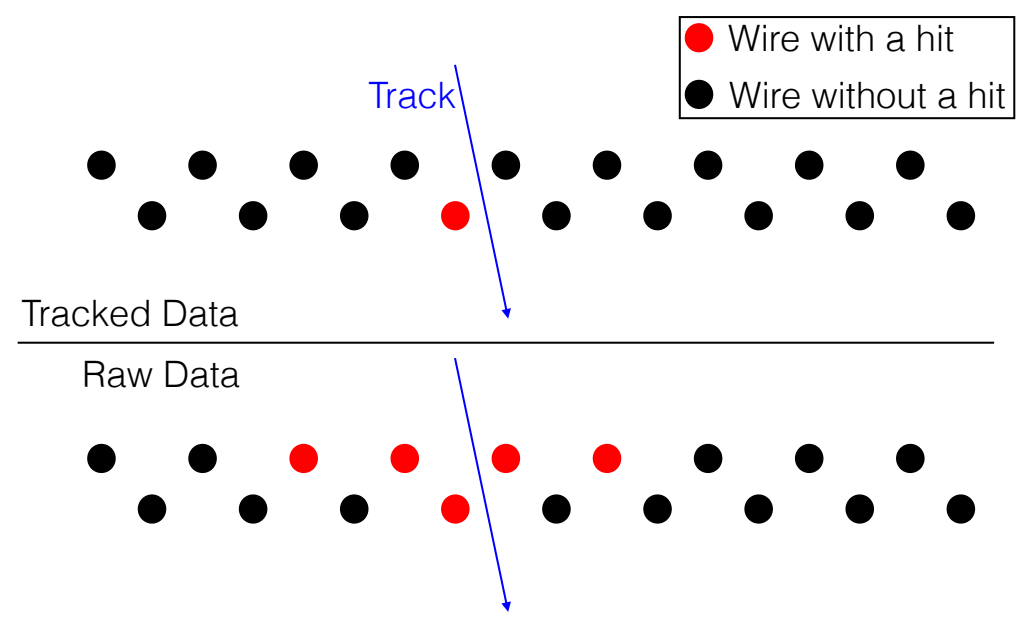

Figure 4.3: Drawing on the method of checking whether the true hits are removed by the hit removal. Red points show the wires with hits. Black points show the wires without hit. Blue arrows are muon tracks. 


\subsubsection{Occupancy Cut}

Some events include too many hits even after the hit removal. In order to remove such events, the chamber occupancy cut is applied. Here, "occupancy" is the number of hits in each drift chamber after the hit removal. Cuts on the occupancy are listed in Tab. 4.2. The events which satisfy the

Table 4.2: Upper limits of the occupancy of each drift chamber.

\begin{tabular}{llc}
\hline Detector & Occupancy & Number of total sense wires \\
\hline \hline St. 1 & 320 & 1124 \\
St. 2 & 160 & 736 \\
St. 3+ & 150 & 768 \\
St. 3- & 150 & 768 \\
\hline
\end{tabular}

occupancy cut are reconstructed.

Figure 4.4 shows the occupancy on the St. $3+$ drift chamber with small amount of real data, for example. The red distribution is the occupancy distribution before the track reconstruction. The blue

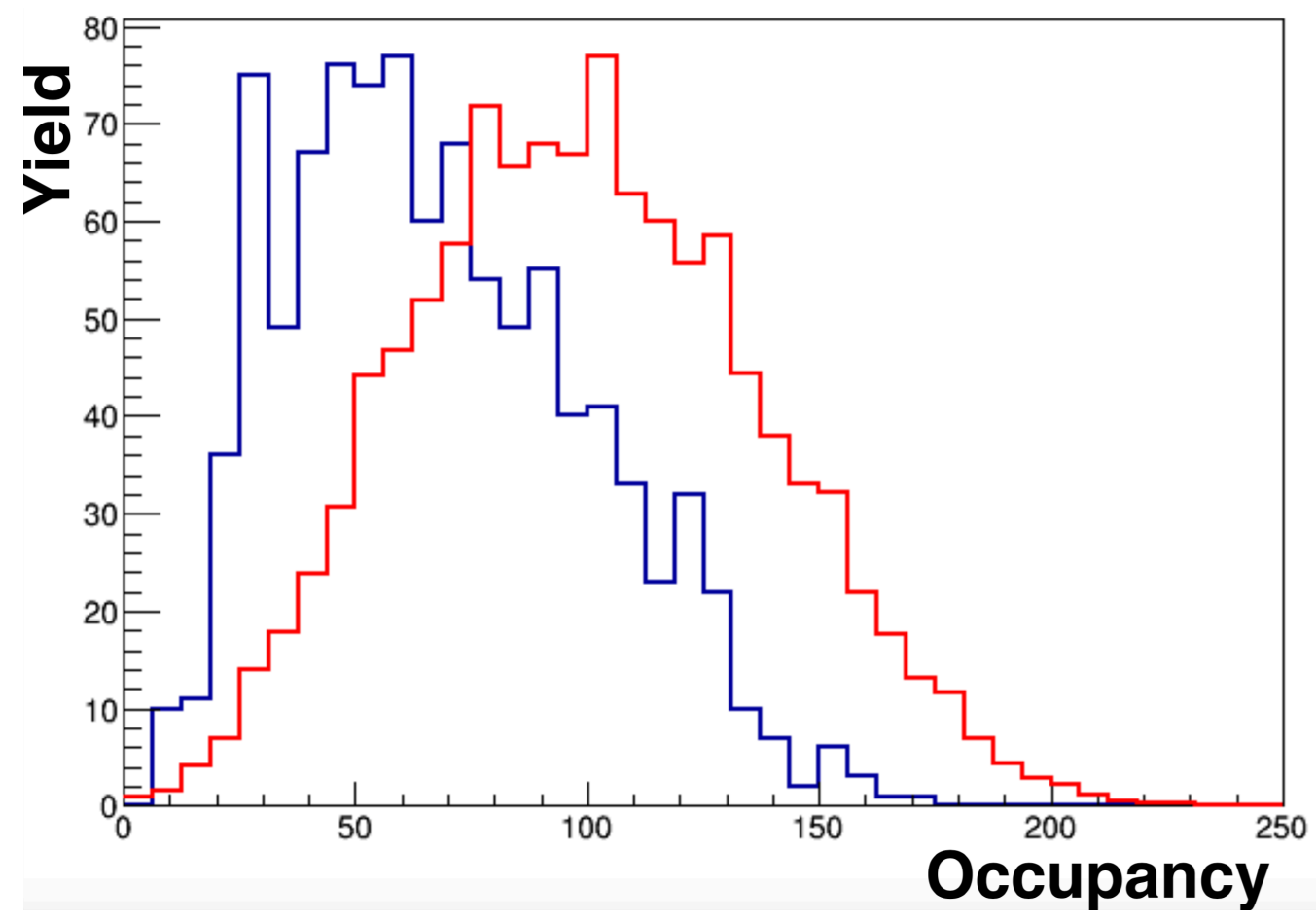

Figure 4.4: Distributions of the occupancy on St. 3+ drift chamber before and after the track reconstruction. The red distribution is the occupancy distribution before the track reconstruction. The blue distribution is the occupancy distribution of the events which survided after the track reconstruction. The red distribution is normalized with the number of events in the blue distribution.

distribution is the occupancy distribution of the events which survided after the track reconstruction. The occupancy cuts are not applied in the track reconstruction. According to the blue histogram, 
the high occupancy events don't have dimuons because no dimuons are reconstructed. The cut-off occupancy is set to be approximately maximum occupancy of the blue distribution in order not to discard dimuons and to sufficiently suppress the processing time.

\subsubsection{Single Track Reconstruction}

Single muon tracks are reconstructed in this stage. This stage includes the following steps:

1. Build tracklets in St. 2 and St. 3

2. Connect tracklets in St. 2 and St. 3

3. Construct global tracks

\subsubsection{Building Tracklets}

A tracklet is literally a small track and is defined as the local track inside each drift chamber. Each drift chamber has six planes, namely $\mathrm{X}, \mathrm{X}^{\prime}, \mathrm{V}, \mathrm{V}^{\prime}, \mathrm{U}$ and $\mathrm{U}^{\prime}$. This method of using tracklet is applied for all the drift chambers since the structure of each drift chamber is similar. The rough structure of a drift chamber is shown in Fig. 4.5.

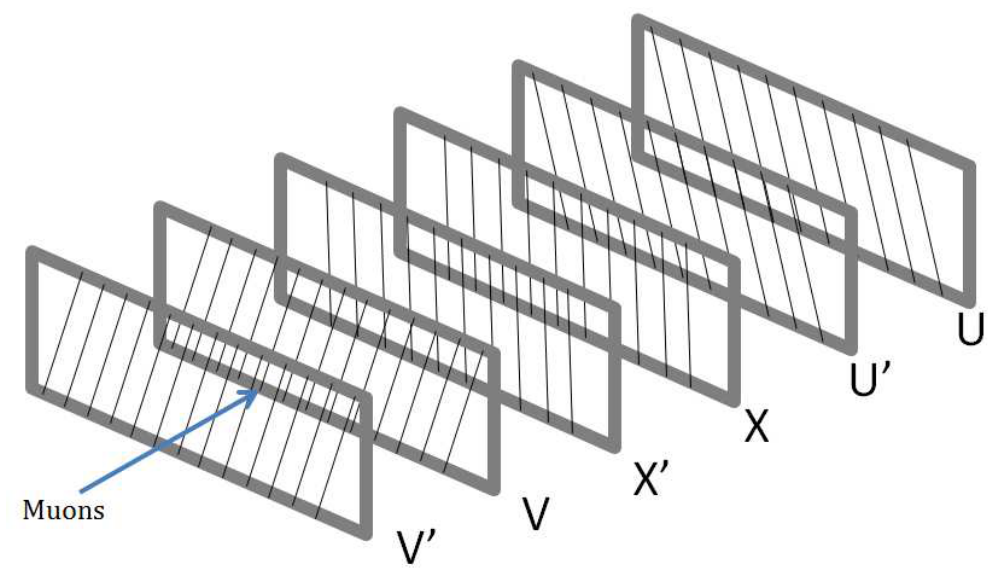

Figure 4.5: Rough structure of drift chamber. It has six planes: $\mathrm{X}, \mathrm{X}^{\prime}, \mathrm{V}, \mathrm{V}^{\prime}, \mathrm{U}$ and $\mathrm{U}^{\prime}$.

The track finding starts from $\mathrm{XX}^{\prime}$ planes. If the difference of an element IDs of a hit on $\mathrm{X}$ and another hit on $\mathrm{X}^{\prime}$ is one or less, the two hits construct a hit pair. The hits which don't construct hit pairs are also treated as "hit pairs". The next step is to find UU' planes hit pairs. The hit pairs are searched in the "window" determined by $\mathrm{XX}^{\prime}$ planes hit pairs. The center of the window is

$$
u_{\text {center }}=x \cos \theta
$$

where $\theta$ is the tilting angle of the $\mathrm{U}$ plane and $x$ is the hit wire position on the $\mathrm{X}$ plane. The width of the window is defined as

$$
u_{\mathrm{win}}=\frac{1}{2} L_{x} \sin \theta+t_{x}^{\max }\left|z_{u}-z_{x}\right| \cos \theta+t_{y}^{\max }\left|z_{u}-z_{x}\right| \sin \theta+2 \Delta u+\delta,
$$




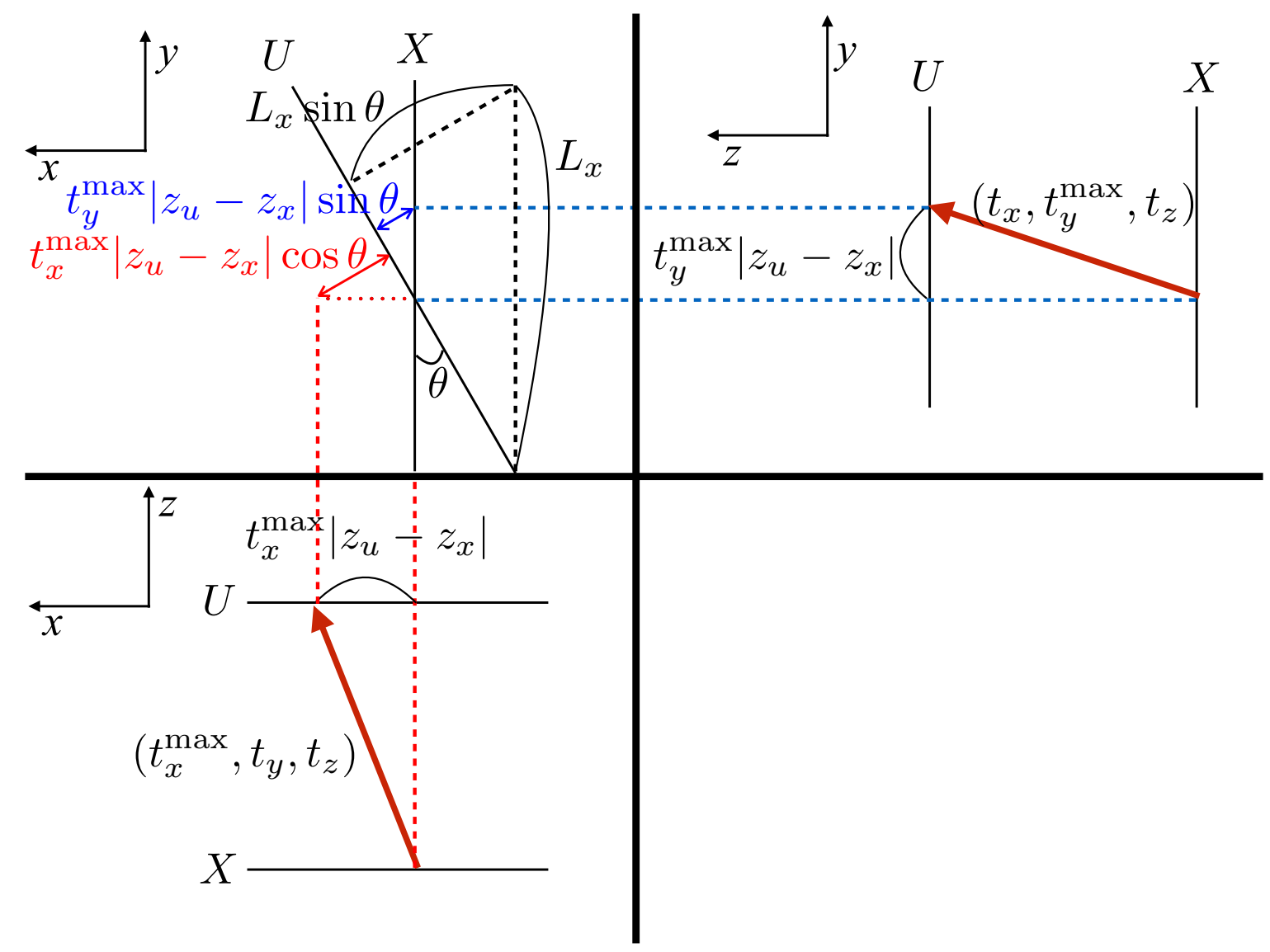

Figure 4.6: Components of $u_{\text {win }}$.

where $L_{x}$ is the length of $\mathrm{X}$ wire, $\Delta u$ is wire spacing of the $\mathrm{U}$ plane, $t_{x}^{\max }(=0.15)$ and $t_{y}^{\max }(=0.1)$ are the maximum tracklet slopes in X-Z and Y-Z views, respectively, $z_{u}$ and $z_{x}$ are $z$ positions of $\mathrm{U}$ and X planes, respectively, and $\delta(=5 \mathrm{~cm}$ for St. 1 and St. 2, $=10 \mathrm{~cm}$ for St. 3 drift chambers) is the extra contingency window. Figure 4.6 shows the components of $u_{\text {win }}$. The window covers the whole wires on $U$ plane which overlap with the hit wire on X plane $\left(L_{x} \sin \theta / 2\right)$. The track slopes $t_{x}$ and $t_{y}$ are taken into account. The effects of the $t_{x}$ and $t_{y}$ are estimated with the maximum $t_{x}$ and $t_{y}$ $\left(t_{x}^{\max }\left|z_{u}-z_{x}\right| \cos \theta\right.$ and $\left.t_{y}^{\max }\left|z_{u}-z_{x}\right| \sin \theta\right)$. All the combinations of hit pairs on $\mathrm{X}$ and $\mathrm{U}$ planes are held if the hits on $U$ plane are in the range of $u_{\text {center }} \pm u_{\text {win }}$ as shown in Fig. 4.7. The third step is to find hit pairs on $\mathrm{VV}^{\prime}$ planes for each tracklet constructed from $\mathrm{X}$ and $\mathrm{U}$ hit pairs. Following relations are satisfied based on Fig. 4.8 if the track slopes in $\mathrm{Y}-\mathrm{Z}$ and $\mathrm{X}-\mathrm{Z}$ planes are known:

$$
\begin{gathered}
u=x \cos \theta+t_{x}\left(z_{u}-z_{x}\right) \cos \theta+t_{y}\left(z_{u}-z_{x}\right) \sin \theta \\
v=x \cos \theta+t_{x}\left(z_{v}-z_{x}\right) \cos \theta-t_{y}\left(z_{v}-z_{x}\right) \sin \theta
\end{gathered}
$$

The sign of third term in Eq. (4.4) is opposite to that in Eq. (4.3). This is because the tilting angle of V plane is opposite to that of U plane. Based on Eq. (4.4) and Eq. (4.3), following relation should be satisfied for a correct combination of hit pairs:

$$
u+v=2 x \cos \theta+t_{x}\left(z_{u}-z_{x}+z_{v}-z_{x}\right) \cos \theta+t_{y}\left(z_{u}-z_{v}\right) \sin \theta
$$




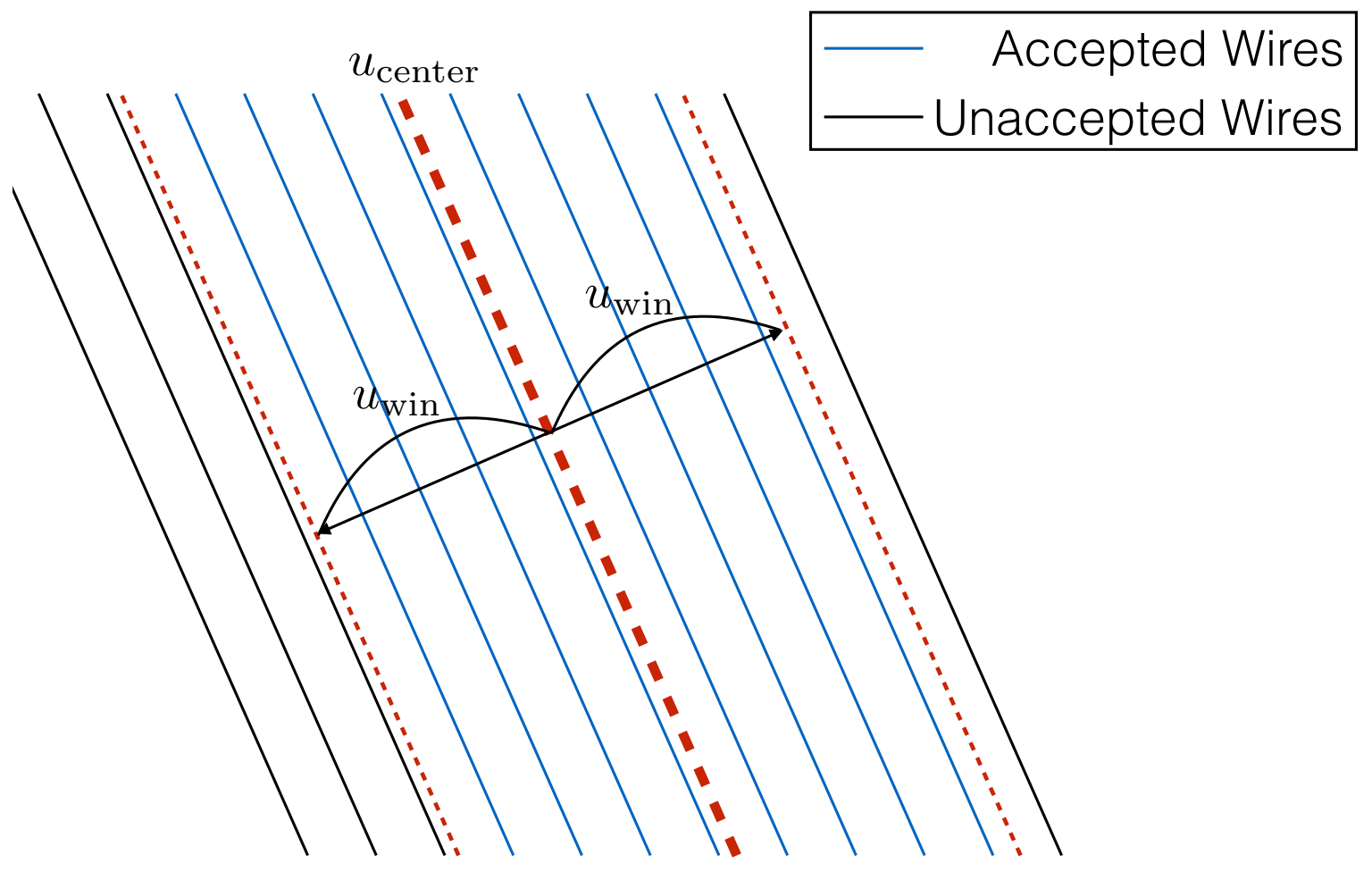

Figure 4.7: Accepted range of $U$ wires. The thick red dotted line is the center position of the window. The thin red dotted lines are the edges of the window. The wires between the thin dotted lines are used for the track reconstruction.

The search window for V plane is calculated based on Eq. (4.5):

$$
\begin{aligned}
& v_{\text {center }}=2 x \cos \theta-u, \\
& v_{\text {win }}=t_{x}^{\max }\left|z_{u}+z_{v}-2 z_{x}\right| \cos \theta+t_{y}^{\max }\left|z_{u}-z_{v}\right| \sin \theta+2 \Delta v,
\end{aligned}
$$

where $x$ and $u$ stand for the hit positions on $\mathrm{X}$ and $\mathrm{U}$ planes in measuring direction, and $\Delta v$ stands for the wire spacing in $\mathrm{V}$ view.

A tracklet is described with four parameters: slopes of the tracklet in $\mathrm{X}-\mathrm{Z}\left(t_{x}\right)$ and $\mathrm{Y}-\mathrm{Z}\left(t_{y}\right)$ views, and the projection of the tracklet at $z=0$ in $x$ component $\left(x_{0}\right)$ and $y$ component $\left(y_{0}\right)$. A $\chi^{2}$ fit is applied to each tracklet using these parameters and assuming the resolution $(r)$ to be the wire spacing divided by $\sqrt{12}$ :

$$
r=\sqrt{\int_{x_{0}-a / 2}^{x_{0}+a / 2} \frac{\left(x-x_{0}\right)^{2}}{a} d x}=\frac{a}{\sqrt{12}},
$$

where $a$ is the wire spacing and $x_{0}$ is the wire position. After the fitting, following selections are applied to remove bad quality tracklets:

- $\left|t_{x}\right|<t_{x}^{\max },\left|t_{y}\right|<t_{y}^{\max },\left|x_{0}\right|<x_{0}^{\max },\left|y_{0}\right|<y_{0}^{\max }$,

- $\chi^{2}<15$

- $N_{\text {hits }}>4$, and $N_{\text {hits }}$ on each $\mathrm{X}, \mathrm{U}, \mathrm{V}$ view should be 1 or 2 , 


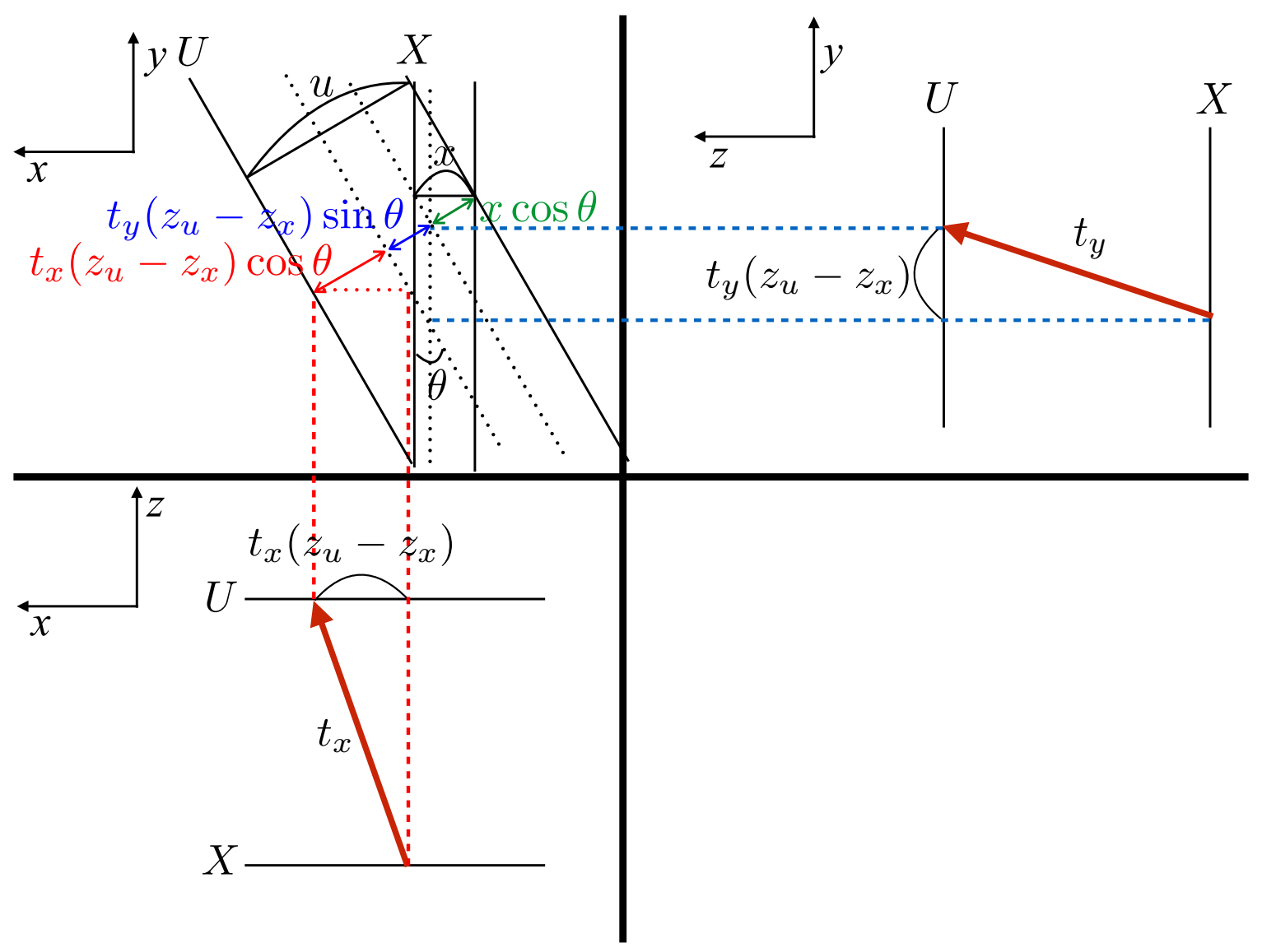

Figure 4.8: Calculation of $u$ hit position based on $x$ hit position. $x$ is hit position in X plane. $u$ is hit position in $\mathrm{U}$ plane. The hit position on $\mathrm{U}$ plane is calculated by adding up $x$ projected to $\mathrm{U}$ plane and the trajectory of the track between $\mathrm{U}$ and $\mathrm{X}$ plane projected to $\mathrm{U}$ plane. 
where $N_{\text {hits }}$ is the number of hits in a tracklet. Here, $\chi^{2}$ is calculated with

$$
\chi^{2}=\sum_{\text {hits }} \frac{\left(w_{h}-w_{t}\right)^{2}}{r}
$$

where $w_{h}$ is the hit wire position, $w_{t}$ is the track position and $r$ is the plane position resolution.

\subsubsection{Connecting Tracklets in St. 2 and St. 3}

The next process is connecting the tracklets. After all the possible tracklets in St. 2 and St. 3 are reconstructed, all the possible combinations of St. 2 and St. 3 tracklets are connected to form a track downstream of KMag. The track which is formed with St. 2 and St. 3 tracklets is named a "back partial track". The hits on X planes in both St. 2 and St. 3 are used for a fast calculation of the X-Z slope $\left(t_{x}^{\text {approx }}\right)$ and intersection $\left(x_{0}^{\text {approx }}\right)$. They should satisfy the following conditions:

- $\left|t_{x}^{\text {approx }}\right|<t_{x}^{\max }$, and

- $\left|x_{0}^{\text {approx }}\right|<x_{0}^{\max }$.

They are also used for a muon identification. The projection of the tracklet to the proportional tubes are performed with $t_{x}^{\text {approx }}$ and $x_{0}^{\text {approx }}$. At least one proportional tube hit on the extrapolated position is required. In addition, the hits on $\mathrm{H} 4 \mathrm{~T}$ and $\mathrm{H} 4 \mathrm{~B}$ at corresponding positions are required. After connecting the St. 2 and St. 3 tracklets, following cuts are applied to remove the bad quality tracks:

- $\left|\left(t_{x}\right)_{\text {St.2 }}-\left(t_{x}\right)_{\text {St. } 3}\right|<0.15$, and

- $\left|\left(t_{y}\right)_{\mathrm{St} .2}-\left(t_{y}\right)_{\mathrm{St} .3}\right|<0.1$,

where $\left(t_{x(y)}\right)_{\text {St. 2(St. 3) }}$ is a slope of a tracklet of St. 2 (St. 3) in X-Z (Y-Z) plane. A $\chi^{2}$ fit is applied to the remaining tracks and then the bad tracks are removed with a selection of $\chi^{2}<3000$. This $\chi^{2}$ selection is rough because the track may include "bad hits". A better $\chi^{2}$ selection is applied at the end of this step. The definition of a "bad hit" is that the residual of the hit is larger than triple wire resolution. In this step, a "bad hit" is removed from the track. After removing a bad hit, a $\chi^{2}$ fit is applied again. The steps of "removing a bad hit" and " $\chi^{2}$ fit" are applied iteratively until all the bad hits are removed from the track.

For a tracklet of St. 3, only one St. 2 tracklet should be connected and alive. The best $\chi^{2}$ track among the tracks which have a common St. 3 tracklet remains and then the others are discarded. Then following quality cuts are applied:

- $\chi^{2}<30$

- $\left|t_{x}\right|<t_{x}^{\max },\left|t_{y}\right|<t_{y}^{\max }$,

- $\left|x_{0}\right|<x_{0}^{\max },\left|y_{0}\right|<y_{0}^{\max }$.

At this stage, similar tracks are discarded: if one third of hits in a track are common in another track, then the two tracks are regarded as the same track. Then, the worse $\chi^{2}$ track is discarded. 


\subsubsection{Constructing Global Tracks}

The next step is connecting the tracks from downstream to upstream of the magnet. Each back partial track is projected back to St. 1 drift chamber through KMag to pair with an appropriate St. 1 tracklet. The track which is formed with St. 1 tracklet and back partial track is named a global track. The search window for St. 1 is determined by the sagitta ratio of St. 1 to St. $2\left(s_{1} / s_{2}\right)$ to be $1.85 \pm 0.25$. The definition of sagittas on St. 1 and St. 2 is shown in Fig. 4.9. The sagittas depend on the momentum

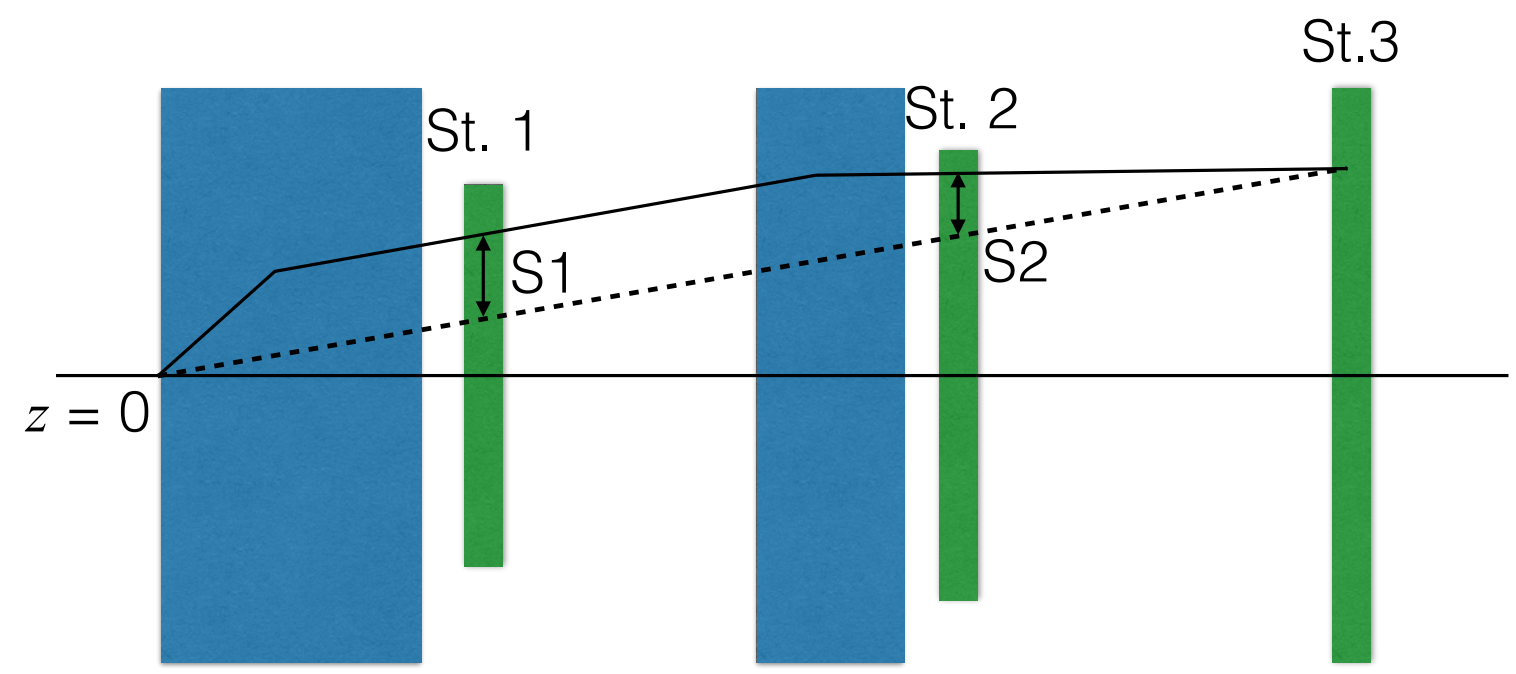

Figure 4.9: Definition of sagitta on St. 1 (S1) and St. 2 (S2).

of the track, but the effect of the momentum is cancelled out by taking ratio. Thus, the sagitta ratio is independent of the track momentum. The sagitta ratio is determined with Monte-Carlo simulation.

All the possible St. 1 tracklets are connected to the back partial track and construct a global tracks candidate.

The $\chi^{2}$ fit is applied to each candidate track. The track is described with five parameters: $s / p_{x z}, t_{x}$, $t_{y}, x_{0}, y_{0}$, where $s$ is charge of the track, and $p_{x z}=\sqrt{p_{x}^{2}+p_{y}^{2}}, t_{x}, t_{y}, x_{0}$ and $y_{0}$ are the momenta, slopes and intersections in $\mathrm{X}-\mathrm{Z}$ and $\mathrm{Y}-\mathrm{Z}$ planes of the back partial track in the track. Note that the slope and intersection in Y-Z plane before KMag are the same as those after KMag because the direction of the magnet field is parallel to Y-axis. The slope and intersection in X-Z plane before KMag are calculated by the following formula using a $p_{T}$ kick:

$$
\begin{aligned}
& t_{x}^{\text {St. } 1}=t_{x}+\operatorname{kick} \frac{s}{p_{x z}}, \\
& x_{0}^{\text {St. } 1}=t_{x} z_{\text {bend }}+x_{0}-t_{x}^{\text {St. } 1} z_{\text {bend }},
\end{aligned}
$$

where kick $=0.4016 \mathrm{GeV}$ and $z_{\text {bend }}=1064.26 \mathrm{~cm}$ is the $z$-position of the center of KMag. The values of kick and $z_{\text {bend }}$ are determined with Monte-Carlo simulation. The steps of "remove a bad hit" and " $\chi^{2}$ fit" are iteratively applied until all the bad hits are removed as is done at the stage of building back partial tracks.

For one back partial track, only one St. 1 tracklet should be connected and alive. The best $\chi^{2}$ global track among the global tracks which have a common back partial track remains and the others are discarded. 
Then, the following quality cuts are applied:

- $N_{\text {hits }}>14$

- $5 \mathrm{GeV}<P<100 \mathrm{GeV}$

- $\chi^{2}<20$

\subsubsection{Reaction Vertex Reconstruction}

The reconstruction of reaction vertex is divided into two steps: single track vertex reconstruction and dimuon vertex reconstruction. The dimuons are reconstructed based on the vertex information of the single tracks.

\subsubsection{Single Track Vertex Reconstruction}

The tracks downstream of the St. 1 drift chamber are reconstructed in the single track reconstruction step. The upstream part from FMag to the vertex position is reconstructed at this step. Since the FMag is composed of iron slabs, a muon loses its energy in addition to a bending by the magnetic field at FMag. Those two effects are taken into account.

The constants used in this step are shown in Tab. 4.3. There is a hole of $28 \mathrm{~cm}$ depth at FMag

Table 4.3: Constants used in vertex fitting. These constants of kick, $E_{0}, E_{1}, E_{2}, E_{3}$ and $E_{4}$ are determined with Monte-Carlo simulation. The others are determined with detector survey.

\begin{tabular}{lrl}
\hline Name & Value & Description \\
\hline kick & 2.909 & $p_{T}$ kick at FMag $(\mathrm{GeV})$ \\
$L_{\text {hole }}$ & 27.94 & length of the FMag hole $(\mathrm{cm})$ \\
$R_{\text {hole }}$ & 1.27 & radius of the FMag hole $(\mathrm{cm})$ \\
$L_{\text {FMag }}$ & 502.92 & length of the FMag $(\mathrm{cm})$ \\
$Z_{U}$ & -500 & upstream end $(\mathrm{cm})$ \\
$E_{0}$ & 7.18274 & parameter for energy loss 0 \\
$E_{1}$ & 0.0361447 & parameter for energy loss 1 \\
$E_{2}$ & -0.000718127 & parameter for energy loss 2 \\
$E_{3}$ & $7.97312 \mathrm{e}-06$ & parameter for energy loss 3 \\
$E_{4}$ & $-3.05481 \mathrm{e}-08$ & parameter for energy loss 4 \\
\hline
\end{tabular}

upstream surface, namely $z=0$. Muons do not lose their energy at the hole.

The FMag is divided into 100 slices in the analysis to take the energy loss into account. The drawing of the vertex reconstruction at a FMag slice is schematically shown in Fig. 4.10. The $p_{T}$ kick is performed at the center of each slice:

$$
t_{x 2}=t_{x 1}+s \cdot \frac{\mathrm{kick}}{L_{\mathrm{FMag}}} \cdot \frac{L_{\mathrm{FMag}}}{N_{s}} \cdot \frac{1}{\sqrt{P_{1}^{2}+P_{1}^{2}}}
$$




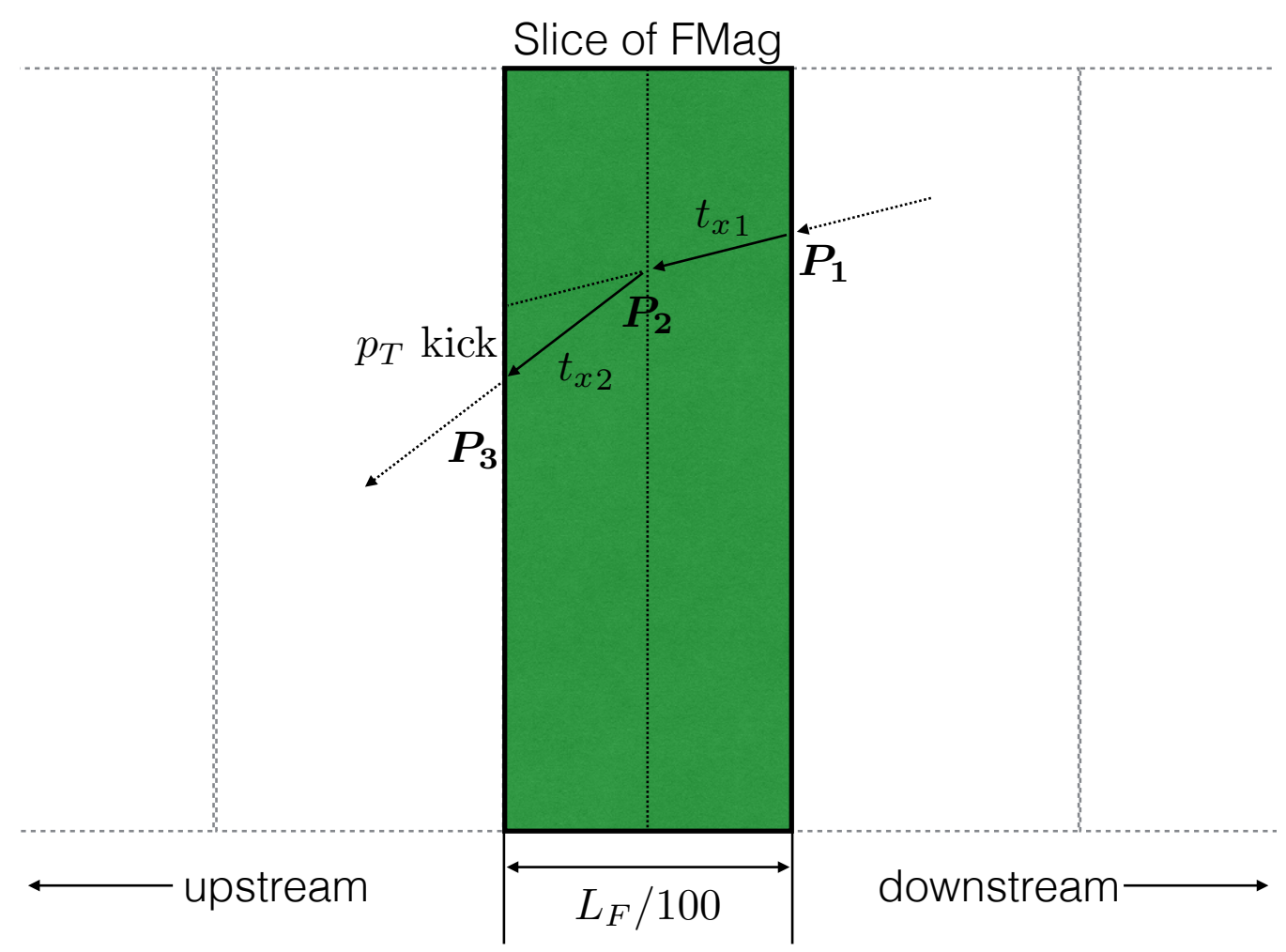

Figure 4.10: Vertex reconstruction at FMag slice.

where $s$ is the sign (charge) of the track, $t_{x 1}$ and $t_{x 2}$ are the track slopes of the track in the X-Z plane at the downstream and upstream surfaces of the FMag slice, respectively, $N_{s}$ is the number of slices in FMag (=100), and $\boldsymbol{P}_{1}$ is the 3-momentum of the track at downstream surface of the FMag slice. The energy loss evaluation is performed twice in each FMag slice: at the former half and latter half of the FMag slice. The 3-momentum at the center of the FMag slice, $\boldsymbol{P}_{2}$, is calculated as

$$
\left|\boldsymbol{P}_{2}\right|=\left|\boldsymbol{P}_{1}\right|+\left(E_{0}+\left|\boldsymbol{P}_{1}\right| \cdot E_{1}+\left|\boldsymbol{P}_{1}\right|^{2} \cdot E_{2}+\left|\boldsymbol{P}_{1}\right|^{3} \cdot E_{3}+\left|\boldsymbol{P}_{1}\right|^{4} \cdot E_{4}\right) \cdot \frac{1}{L_{\mathrm{FMag}}} \cdot \frac{1}{\left|\boldsymbol{T}_{1}\right|},
$$

where $\boldsymbol{T}_{1}$ is the trajectory of the track at the former half of the FMag slice:

$$
\boldsymbol{T}_{1}=\left(\frac{t_{x 1} \cdot L_{\mathrm{FMag}}}{2 \cdot N_{s}}, \frac{t_{y} \cdot L_{\mathrm{FMag}}}{2 \cdot N_{s}}, \frac{L_{\mathrm{FMag}}}{2 \cdot N_{s}}\right) .
$$

The second term of Eq. (4.13) corresponds to the energy loss. As with at the former half, the 3momentum at the upstream end of the FMag slice, $\boldsymbol{P}_{3}$, is

$$
\left|\boldsymbol{P}_{3}\right|=\left|\boldsymbol{P}_{2}\right|+\left(E_{0}+\left|\boldsymbol{P}_{2}\right| \cdot E_{1}+\left|\boldsymbol{P}_{2}\right|^{2} \cdot E_{2}+\left|\boldsymbol{P}_{2}\right|^{3} \cdot E_{3}+\left|\boldsymbol{P}_{2}\right|^{4} \cdot E_{4}\right) \cdot \frac{1}{L_{\mathrm{FMag}}} \cdot \frac{1}{\left|\boldsymbol{T}_{2}\right|},
$$

where $\boldsymbol{T}_{2}$ is the trajectory of the track at the latter half of the FMag slice:

$$
\boldsymbol{T}_{2}=\left(\frac{t_{x 2} \cdot L_{\mathrm{FMag}}}{2 \cdot N_{s}}, \frac{t_{y} \cdot L_{\mathrm{FMag}}}{2 \cdot N_{s}}, \frac{L_{\mathrm{FMag}}}{2 \cdot N_{s}}\right) .
$$

The energy loss evaluation is performed when the position of the track is not at the hole of the FMag: $z>L_{\text {hole }}$ and $\sqrt{x^{2}+y^{2}}>R_{\text {hole }}$. Then the $\boldsymbol{P}_{3}$ is thrown to the next FMag slice as $\boldsymbol{P}_{1}$. Figure 4.11 


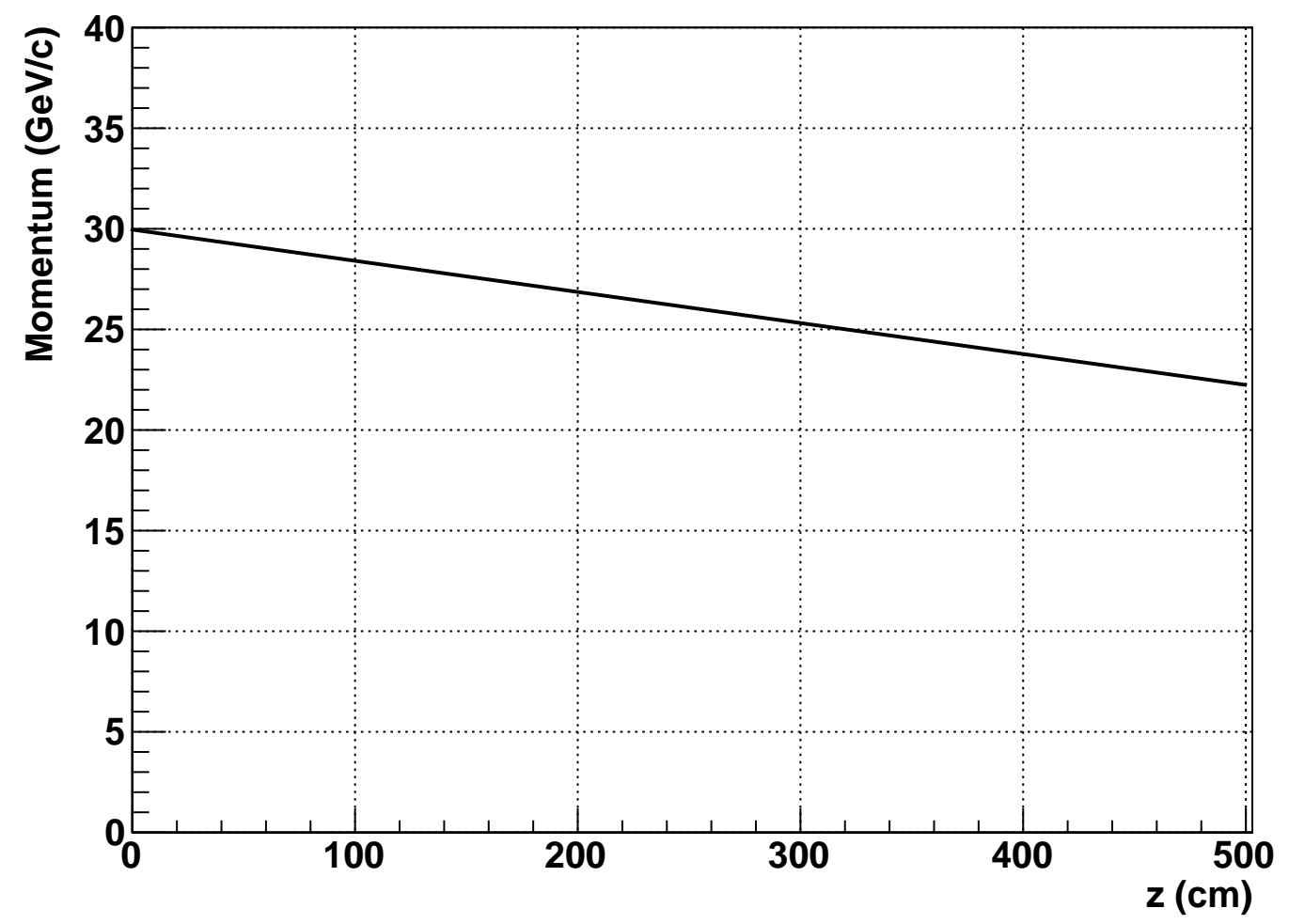

Figure 4.11: Muon momentum variation due to the energy loss at FMag. The initial momentum of the DrellYan muon is $30 \mathrm{GeV} / \mathrm{c}$, which is the typical momentum of the muon. The variation is calculated based on Eq. (4.13). 
shows the momentum variation due to the energy loss in FMag. The initial momentum of the muon is $30 \mathrm{GeV} / \mathrm{c}$, which is the typical momentum of the Drell-Yan muon.

After performing this procedure in the whole FMag, the track is extrapolated to the target position. Although there are no magnetic field nor solid material, the extrapolating procedure is performed in each $2.5 \mathrm{~cm}$ slice in order to evaluate the vertex position. The distance between $z$-axis and the track at each slice is calculated with the vertex position. The $z$-position of the slice whose distance is "DCA" corresponds to the vertex position. The DCA stands for "distance of closest approach", and is defined as the smallest distance between the $z$-axis and the track among the slices. 


\subsubsection{Dimuon Vertex Reconstruction}

The dimuons are reconstructed in this step. First, all the reconstructed single tracks in an event are classified into plus and minus muons: $\mu^{+}$and $\mu^{-}$. All the combinations of $\mu^{+}$and $\mu^{-}$are tested if they construct dimuons. The dimuon vertex position is obtained based on the two track positions at each slice defined in the former subsection. The slice position is the vertex position where the distance between the $z$-axis and the difference of the track positions, $\boldsymbol{P}_{d}=\boldsymbol{P}_{+}-\boldsymbol{P}_{-}$, is smallest. The vertex position is used as the initial parameter of the iterative analysis. Using Kalman-Filter method, the vertex position is updated until the $\chi^{2}$ becomes minimum. The vertex distribution is shown in a data selection.

\subsection{Selections and Quality Cuts}

Quality cuts are applied after the dimuon reconstruction. The quality cuts are classified into two levels: spill level and event level. The dimuons in the events which satisfied those cuts are used in the physics analysis. The flow chart of selections and quality cuts is shown in Fig. 4.12.

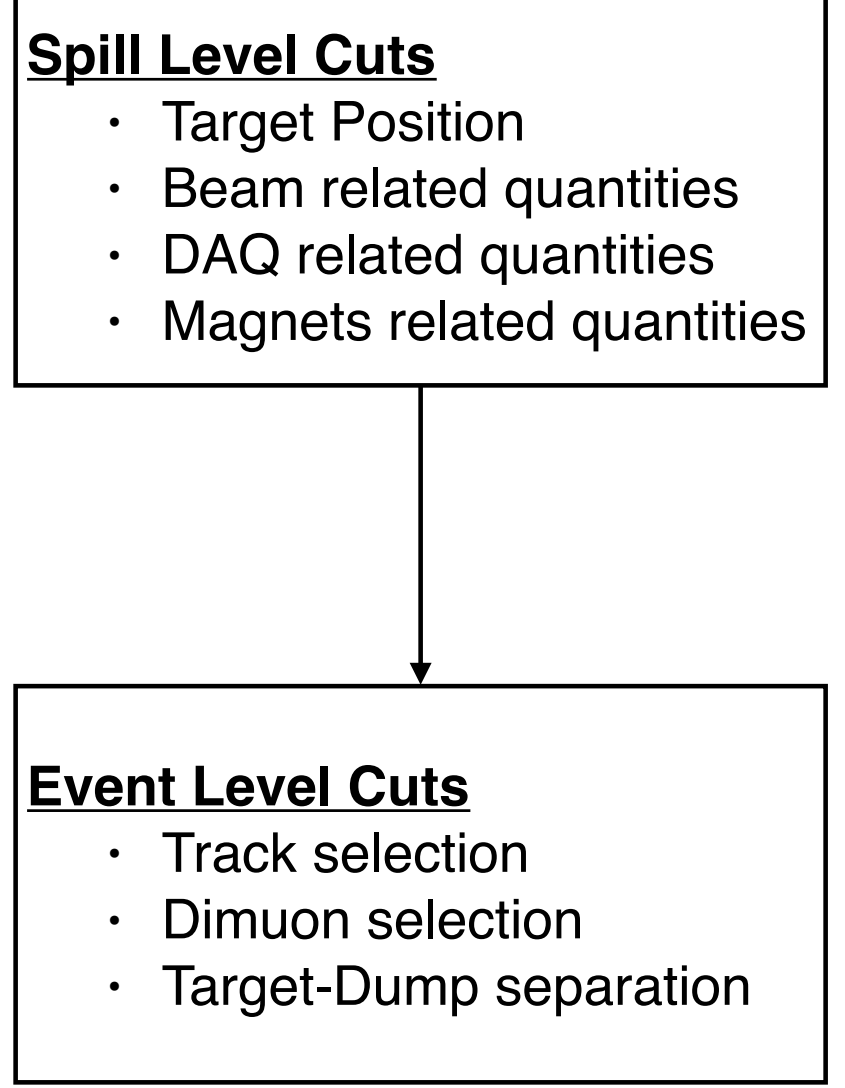

Figure 4.12: Flow chart of selections and quality cuts. 


\subsubsection{Spill Level Selection}

The spill level selections are applied spill-by-spill. The spill level selections are summarized in Tab. 4.4.

Table 4.4: Spill level selections.

\begin{tabular}{lcc}
\hline Quantity & \multicolumn{2}{c}{ Good range } \\
\hline Roadset & 57 & 62,67 and 70 \\
\hline Target Position & {$[1,7]$} & {$[1,7]$} \\
Duty factor & {$[15,60]$} & {$[10,60]$} \\
G2SEM & {$[2 \mathrm{e} 12,1 \mathrm{e} 13]$} & {$[2 \mathrm{e} 12,1 \mathrm{e} 13]$} \\
QIEsum & {$[4 \mathrm{e} 10,1 \mathrm{e} 12]$} & {$[4 \mathrm{e} 10,1 \mathrm{e} 12]$} \\
TSGo & {$[1 \mathrm{e} 3,8 \mathrm{e} 3]$} & {$[100,6000]$} \\
AcceptedFPGA1 & {$[1 \mathrm{e} 3,8 \mathrm{e} 3]$} & {$[100,6000]$} \\
AfterInhFPGA1 & {$[1 \mathrm{e} 3,30 \mathrm{e} 3]$} & {$[100,10000]$} \\
AcceptedFGPA1 / AfterInhFPGA1 & {$[0.2,0.9]$} & {$[0.2,1.05]$} \\
Inhibit & {$[4 \mathrm{e} 9,1 \mathrm{e} 11]$} & {$[4 \mathrm{e} 9,2 \mathrm{e} 11]$} \\
Busy & {$[4 \mathrm{e} 9,1 \mathrm{e} 11]$} & {$[4 \mathrm{e} 9,1 \mathrm{e} 11]$} \\
FMag & $>1000$ & $>1000$ \\
KMag & $>1000$ & $>1000$ \\
Tracks/spill & $>0$ & $>0$ \\
\hline
\end{tabular}

The number of good quality spills and the number of live protons in each roadset are listed in Tab. 4.5.

Table 4.5: The number of good spills and the number of live protons in each roadset.

\begin{tabular}{cccccc}
\hline Roadset & \multicolumn{2}{c}{ Number of good spills } & \multicolumn{3}{c}{ Number of Live Protons } \\
& All & $\mathrm{LH}_{2} / \mathrm{LD}_{2} /$ Empty targets & $\mathrm{LH}_{2}$ & Empty & $\mathrm{LD}_{2}$ \\
\hline 57 & 41,570 & 29,207 & $4.0258 \mathrm{e}+16$ & $4.4732 \mathrm{e}+15$ & $2.0242 \mathrm{e}+16$ \\
62 & 49,015 & 36,078 & $5.4306 \mathrm{e}+16$ & $1.1293 \mathrm{e}+16$ & $2.4443 \mathrm{e}+16$ \\
67 & 98,984 & 71,539 & $1.0729 \mathrm{e}+17$ & $2.5463 \mathrm{e}+16$ & $5.5818 \mathrm{e}+16$ \\
70 & 5,310 & 3,911 & $6.0457 \mathrm{e}+15$ & $1.3019 \mathrm{e}+15$ & $3.0211 \mathrm{e}+15$ \\
\hline
\end{tabular}




\subsubsection{Target Position}

The target position is monitored spill-by-spill. Figure 4.13 shows the target position as a function of the spill ID. Red points are the good spill events and black points are events rejected by the spill-level selections. The targets are numbered from 1 to 7 : $\mathrm{LH}_{2}$, Empty Flask, $\mathrm{LD}_{2}$, None, Iron, Carbon and

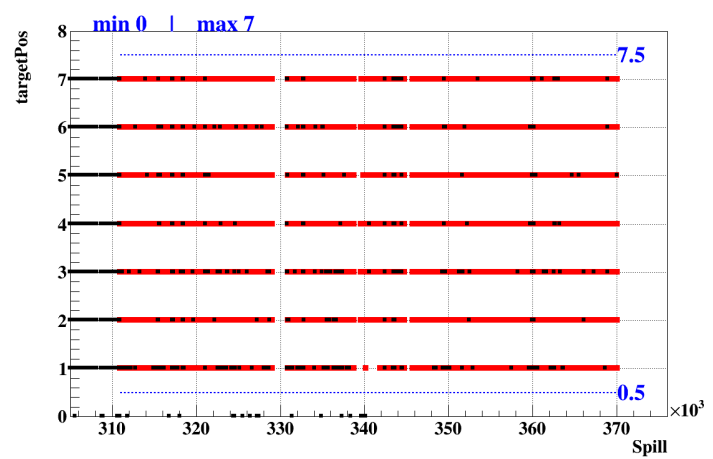

(a) Roadset 57

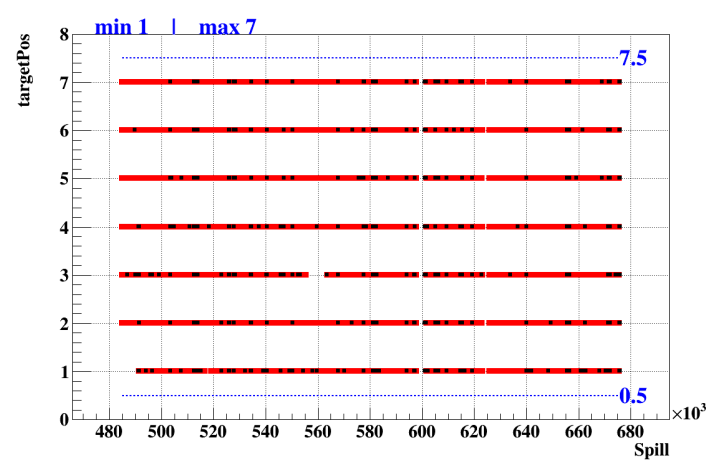

(c) Roadset 67

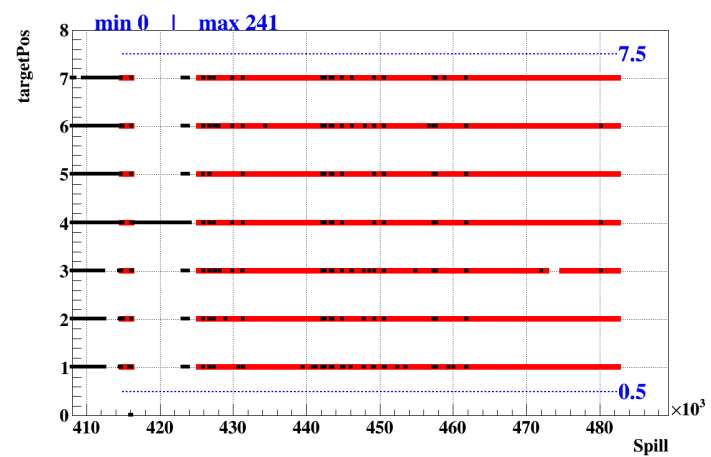

(b) Roadset 62

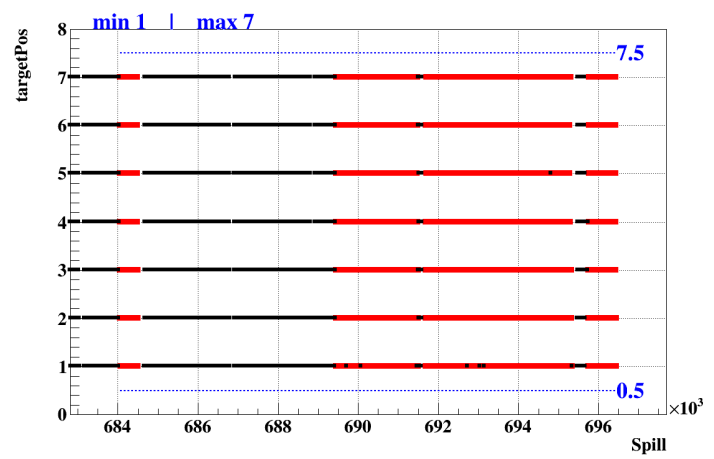

(d) Roadset 70

Figure 4.13: Target position as a function of spill ID. The target positions are numbered from 1 to 7 in each roadset. One spill corresponds to one minute with 5 seconds beam on.

Tungsten. The target position should be in this range. Most events satisfy this target selection. 


\subsubsection{Beam Related Quantities}

"Duty factor" indicates the stability of the beam intensity I in a RF bucket. The definition of the duty factor is

$$
\text { Duty Factor }=\frac{\langle I\rangle^{2}}{\left\langle I^{2}\right\rangle} .
$$

The duty factor shown here is measured by the Cherenkov counter. The beam intensity $I$ is measured RF-bucket-by-RF-bucket, namely $53 \mathrm{MHz}$. Figure 4.14 shows the duty factor as a function of the spill ID. The duty factor increased at the middle of Roadset 62 . The typical duty factor is about $30 \%$

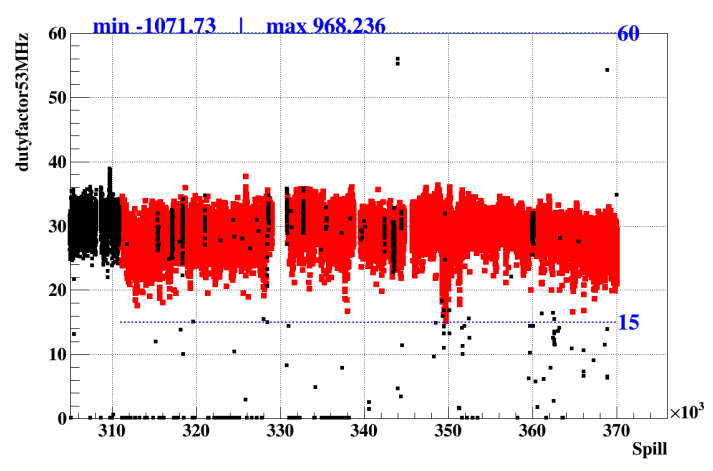

(a) Roadset 57

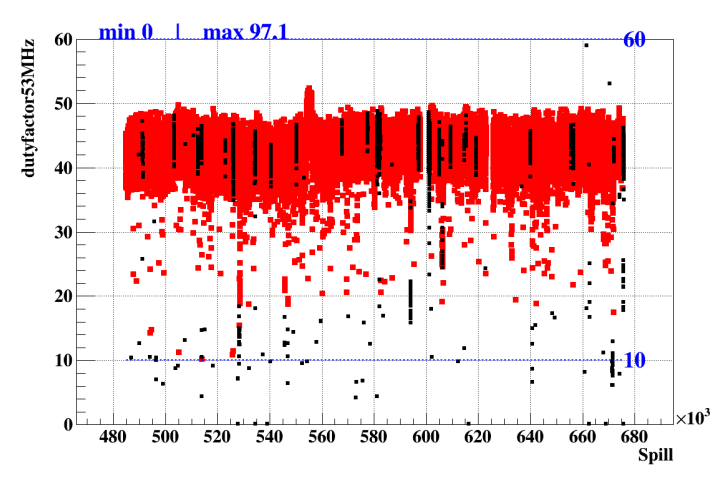

(c) Roadset 67

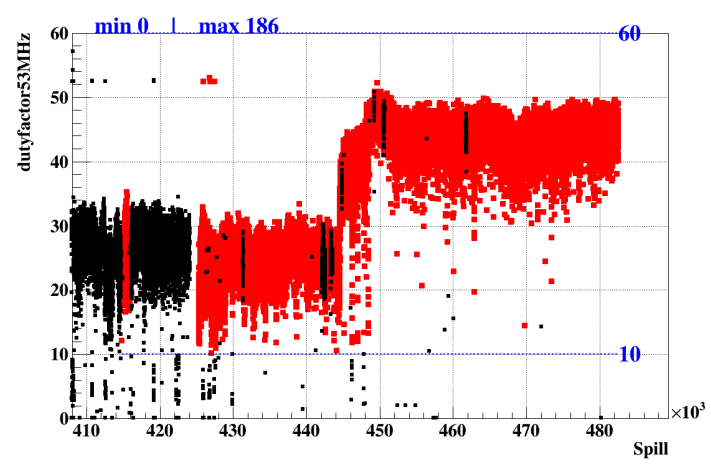

(b) Roadset 62

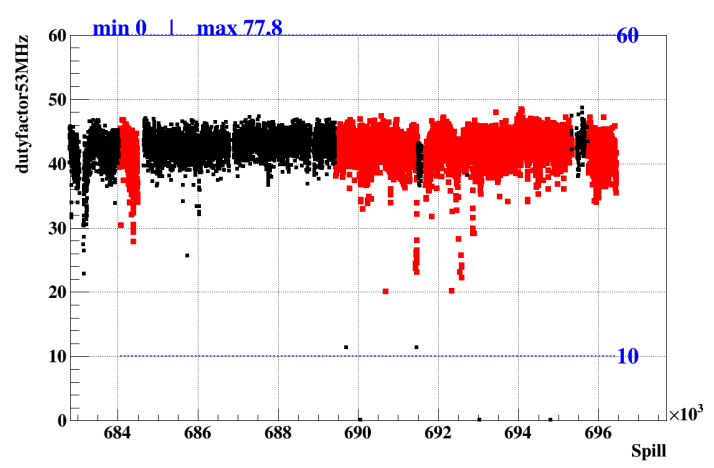

(d) Roadset 70

Figure 4.14: Duty factor as a function of spill ID. Red points are the good spill events and black points are events rejected by the spill-level selections.

before the increase, and is about $40 \%$ after the increase.

"G2SEM" is the number of protons in the spill measured by SEM. Figure 4.15 shows the G2SEM as a function of the spill ID. The typical G2SEM is about $5-7 \times 10^{12}$ protons/spill. The number of beam protons in a spill is sometimes very low: $\sim 10^{9}$ protons/spill. The G2SEM selection removes such kind of spills.

"QIESum" is the relative number of protons in the spill measured by Cherenkov counter. Figure 4.16 shows the QIEsum as a function of the spill ID. The QIEsum decreases gradually in the whole range while the G2SEM does not decreases. This is caused by aging of the mirror of the Cherenkov counter. The role of the mirror is to reflect the Cherenkov light to the PMT. The mirror 


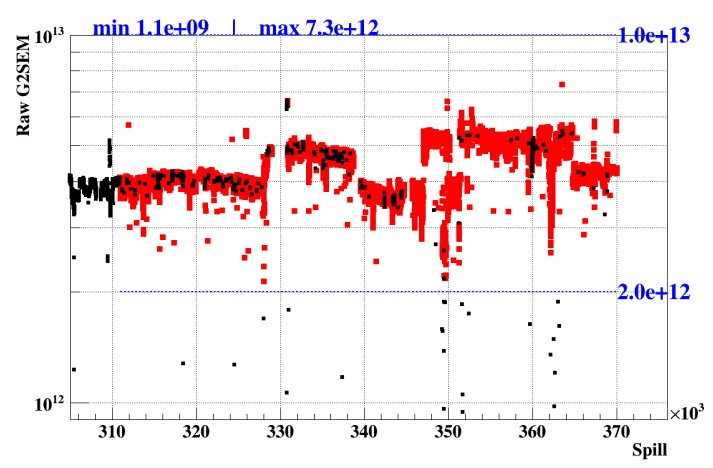

(a) Roadset 57

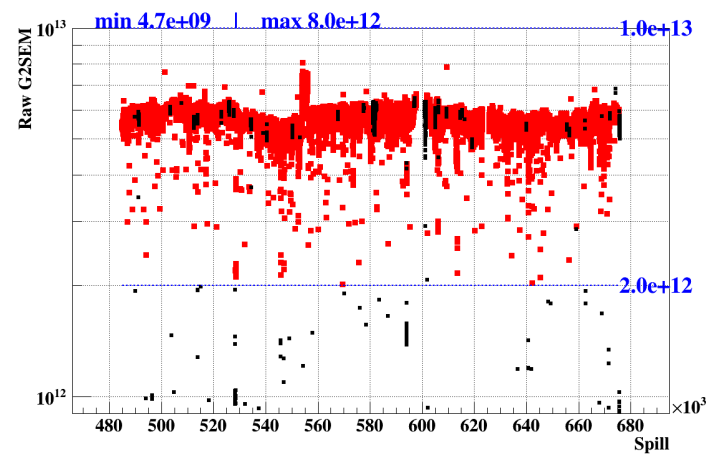

(c) Roadset 67

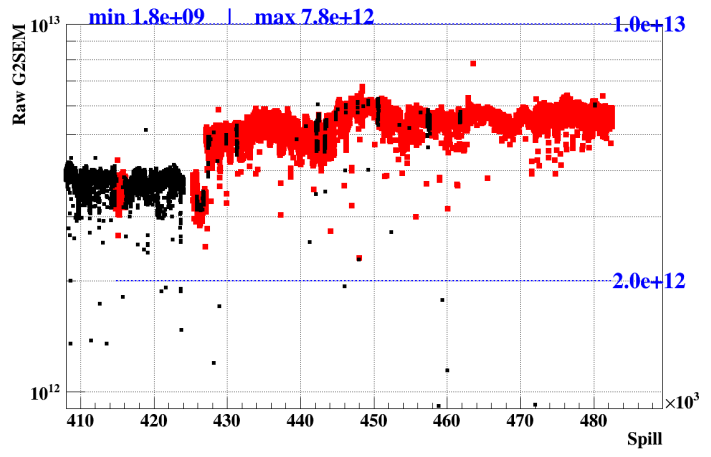

(b) Roadset 62

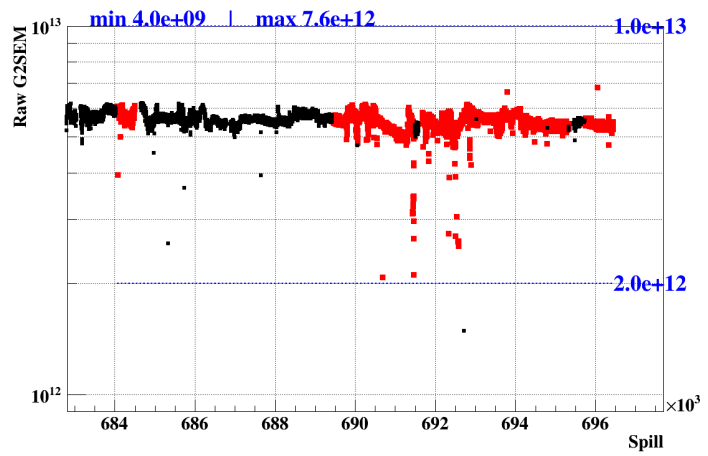

(d) Roadset 70

Figure 4.15: G2SEM as a function of spill ID. Red points are the good spill events and black points are events rejected by the spill-level selections. 


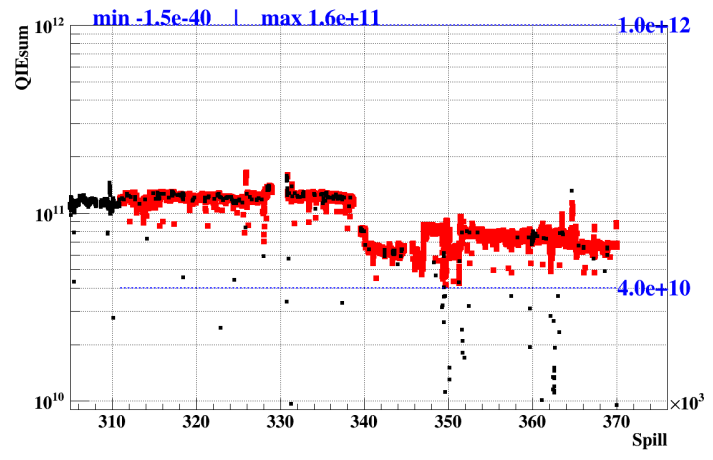

(a) Roadset 57



(c) Roadset 67

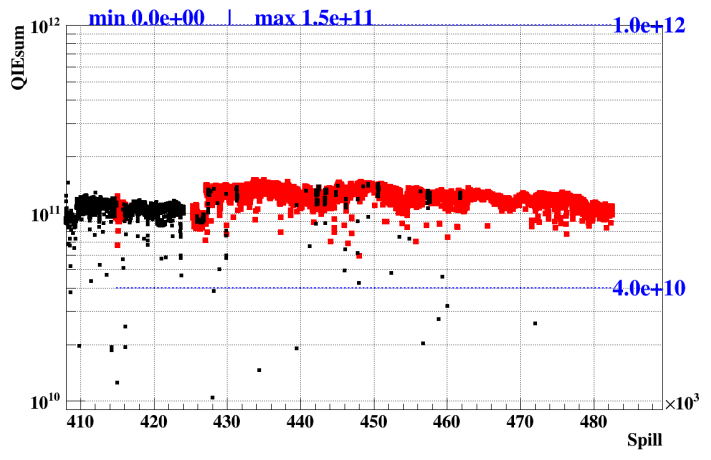

(b) Roadset 62

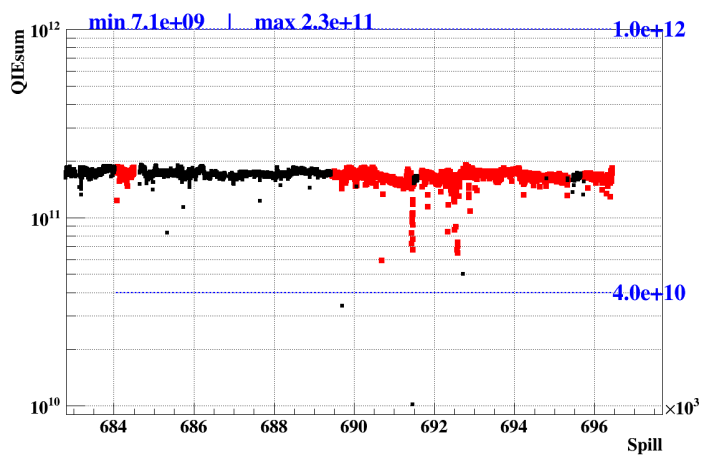

(d) Roadset 70

Figure 4.16: QIEsum as a function of spill ID. Red points are the good spill events and black points are events rejected by the spill-level selections. 
accumulated damages gradually by the beam, and then the reflectance of the beam decreases. The mirrors are sometimes replaced.

\subsubsection{DAQ Related Quantities}

The selection is made also on the DAQ related quantities. The flow chart of data taking in one spill is shown in Fig. 4.17.

"TSGo" is the number of recorded events per spill which are triggered by all the types of triggers including FPGA1 trigger. Figure 4.18 shows the TSGo as a function of spill ID. The typical TSGo is thousands. The TSGo becomes more stable from Roadset 62 because the duty factor of the beam has been better since the middle of Roadset 62 . Therefore, the number of spills which have extraordinary TSGo becomes less from Roadset 62. The accepted ranges of Roadset 62, 67, and 70 are wider than that of Roadset 57.

"AfterInhFPGA1" is the number of events which are triggered by FPGA1 trigger and are not blocked by QIE VETO signal. Figure 4.19 shows the AfterInhFPGA1 as a function of spill ID.

"AcceptedFGPA1" is the number of events which are triggered by FPGA1 trigger and recorded into the database. Figure 4.20 shows the AcceptedFGPA1 as a function of spill ID. The AcceptedFPGA1 is almost the same as TSGo. This is because almost all the events are triggered by the FPGA-1 trigger due to its the prescale factor.

The ratio of the AcceptedFGPA1 to AfterInhFPGA1 is shown in Fig. 4.21. The ratios in Roadsets 62, 67 and 70 are almost 1.0. The ratios in Raodset 57 are lower than those of the other Roadsets. This is because the duty factor in Roadset 57 is lower than that of the other Roadsets. The ratios of some events are low $(<0.2)$ due to the beam quality. These kind of events are discarded.

"Inhibit" is the number of RF-buckets which are blocked by QIE VETO signal (Fig. 4.22). The typical number of Inhibit is about $10^{11}$. The beam intensity in Roadset 57 is lower than that in the other Roadsets. It leads the trigger rate in Roadset 57 to be lower than the others. Therefore, the inhibit in Roadset 57 is lower than that in the other Roadsets.

"Busy" is the number of RF-buckets which are blocked by TS busy signal (Fig 4.23). The typical number of Busy is about $10^{10}$. The selection is made based on this. 


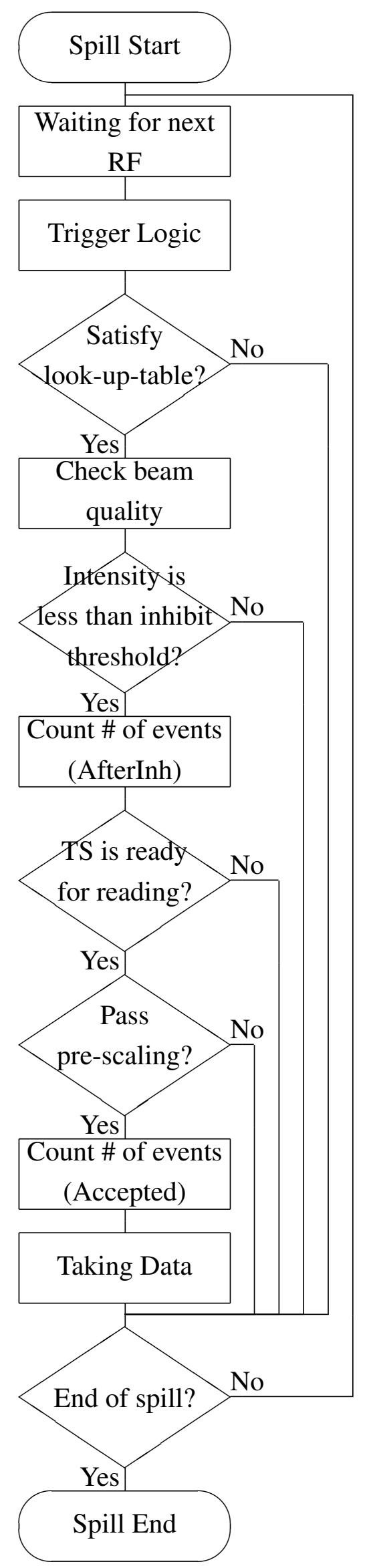

Figure 4.17: Flow chart of data taking in one spill. 


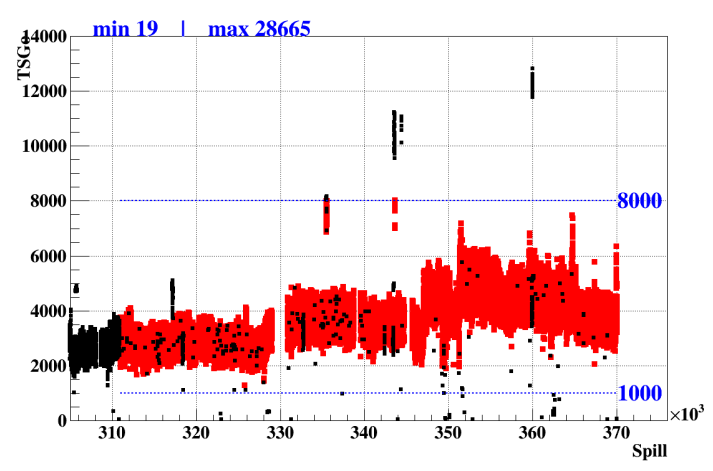

(a) Roadset 57

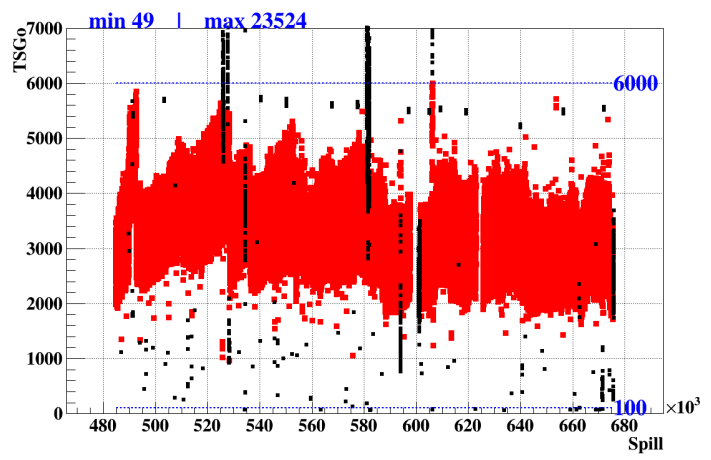

(c) Roadset 67

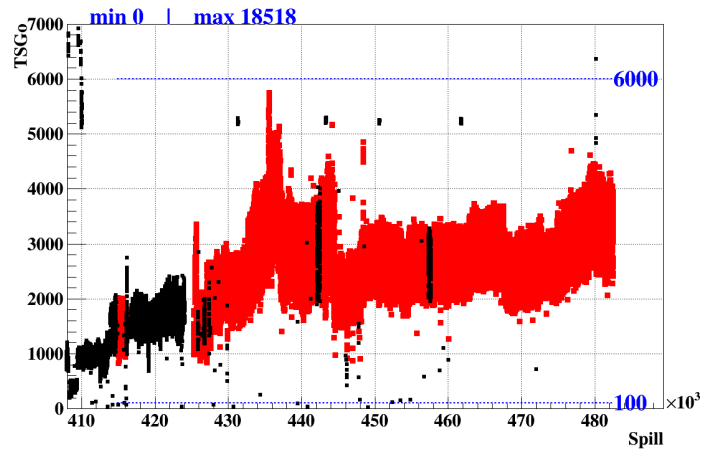

(b) Roadset 62

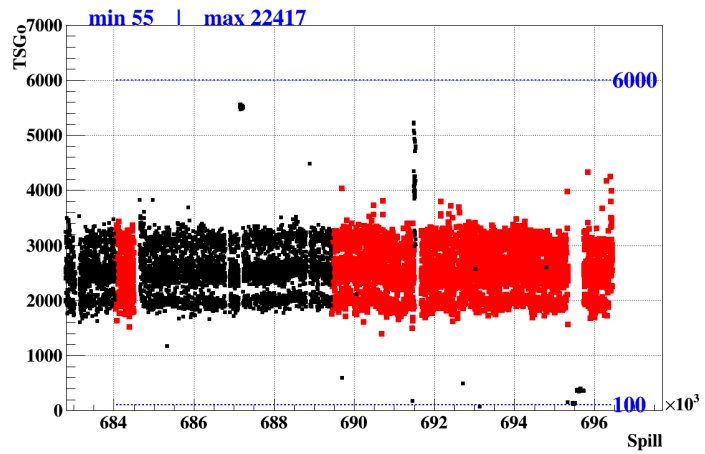

(d) Roadset 70

Figure 4.18: TSGo as a function of spill ID. Red points are the good spill events and black points are events rejected by the spill-level selections. 


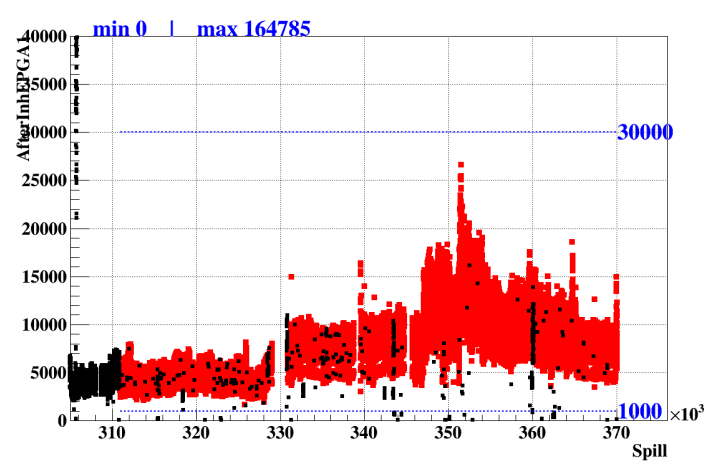

(a) Roadset 57

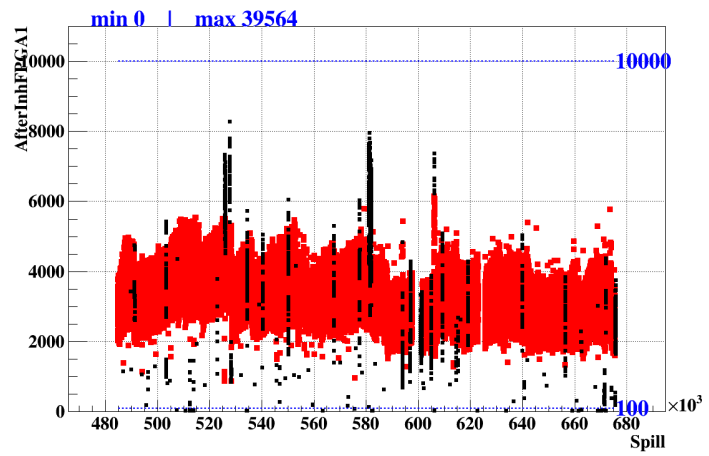

(c) Roadset 67

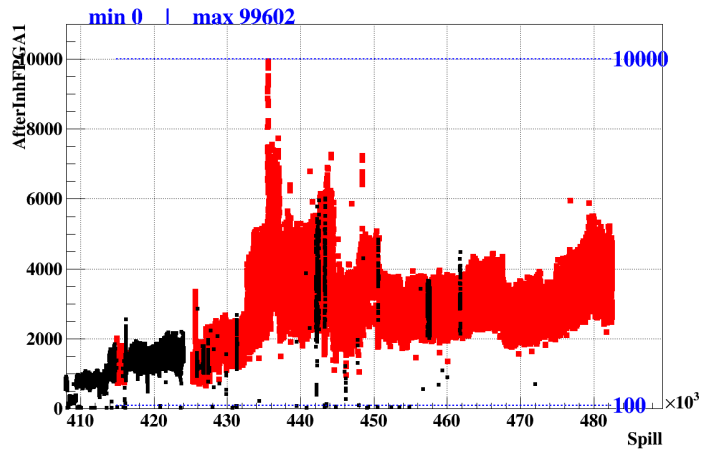

(b) Roadset 62

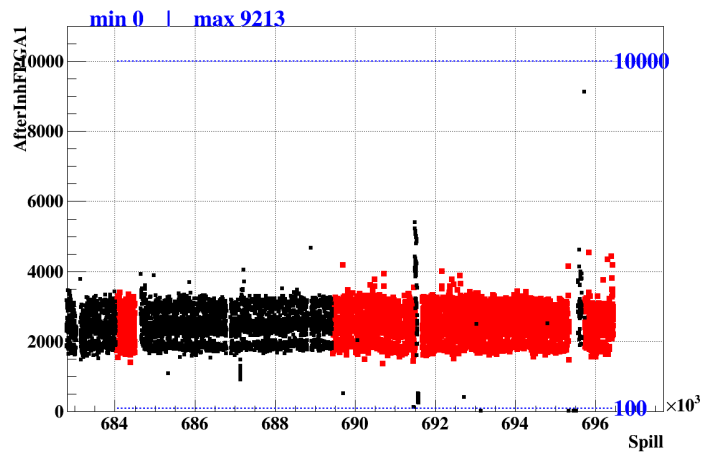

(d) Roadset 70

Figure 4.19: AfterInhFPGA1 as a function of spill ID. Red points are the good spill events and black points are events rejected by the spill-level selections. 


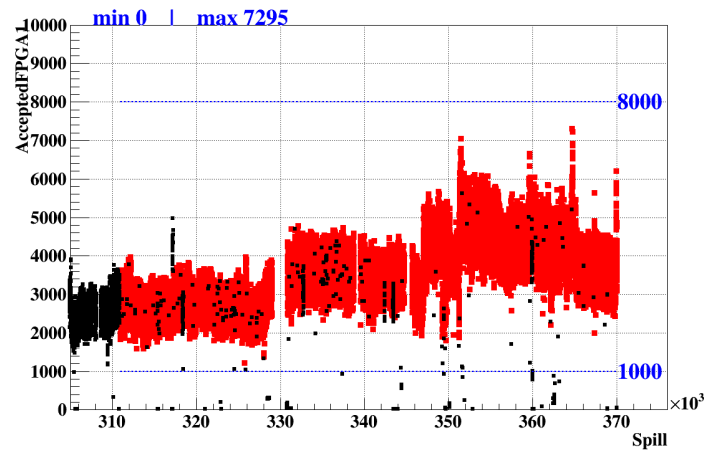

(a) Roadset 57

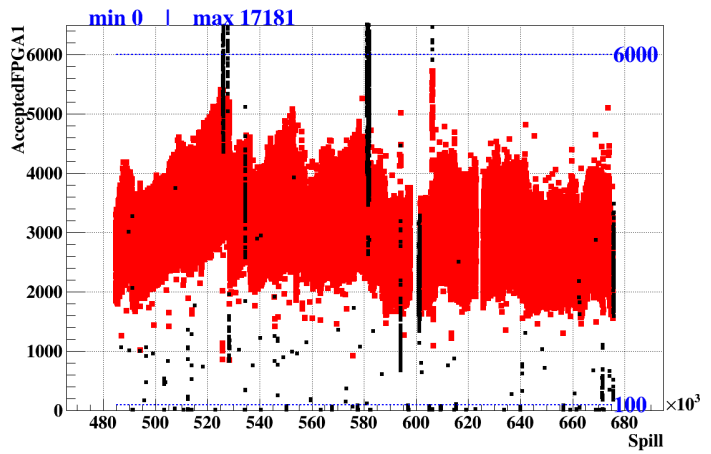

(c) Roadset 67

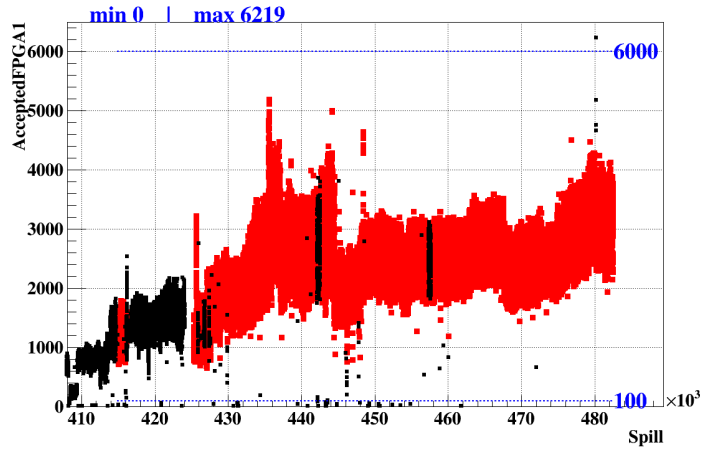

(b) Roadset 62

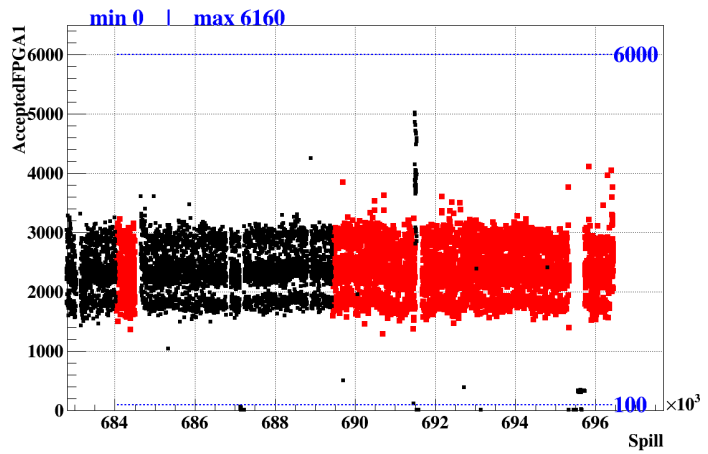

(d) Roadset 70

Figure 4.20: AcceptedFGPA1 as a function of spill ID. Red points are the good spill events and black points are events rejected by the spill-level selections. 


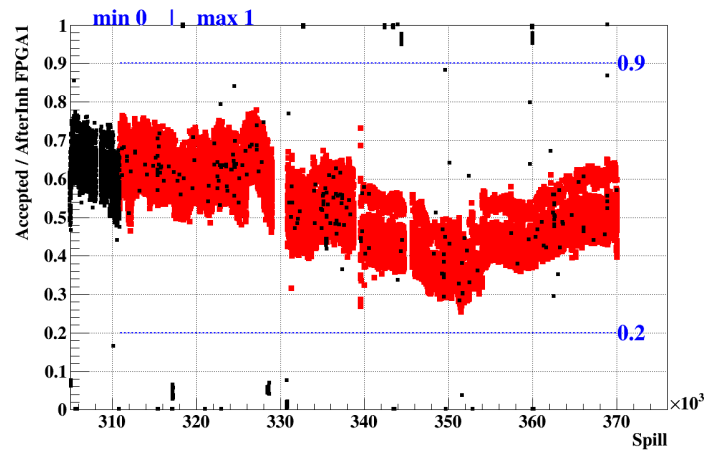

(a) Roadset 57

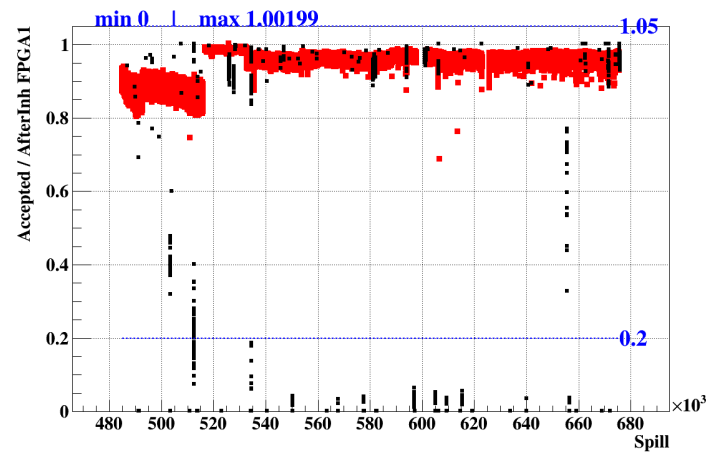

(c) Roadset 67

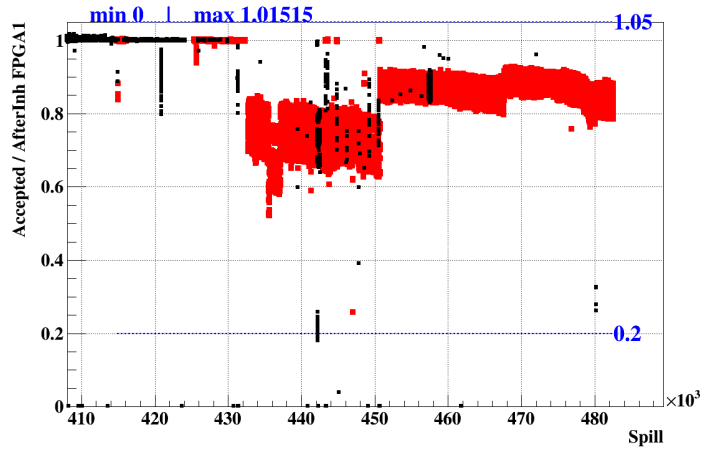

(b) Roadset 62

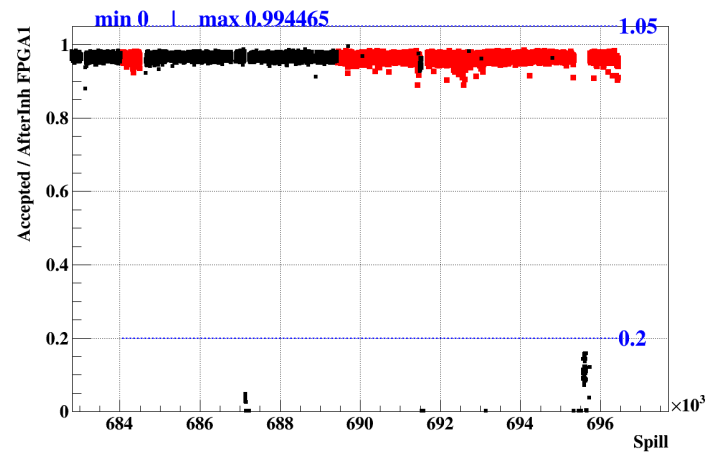

(d) Roadset 70

Figure 4.21: AcceptedFGPA1/AfterInhFPGA1 as a function of spill ID. Red points are the good spill events and black points are events rejected by the spill-level selections. 


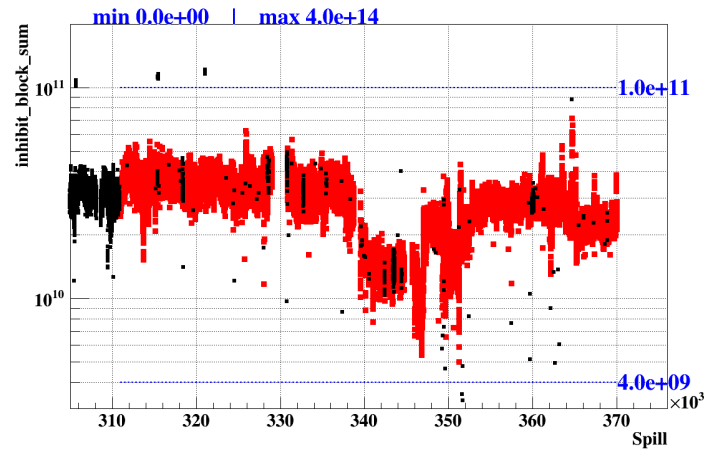

(a) Roadset 57

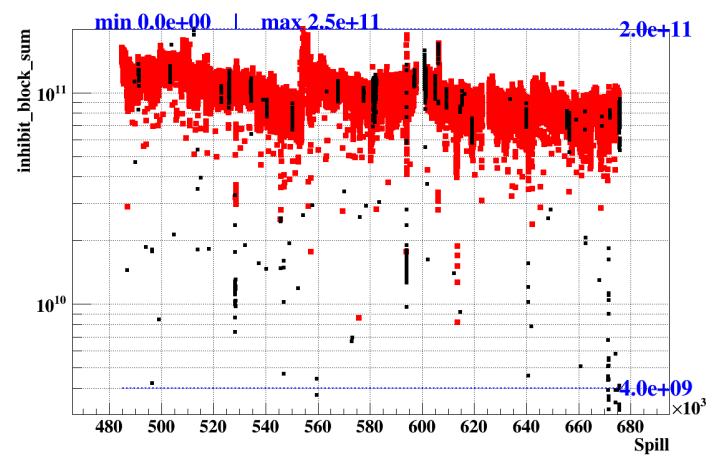

(c) Roadset 67

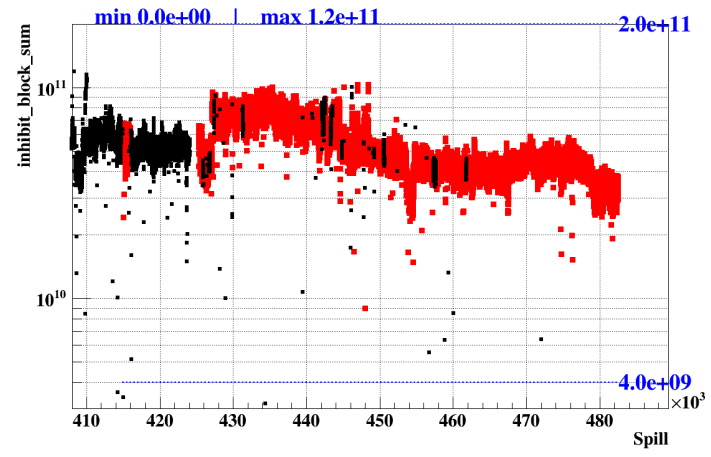

(b) Roadset 62

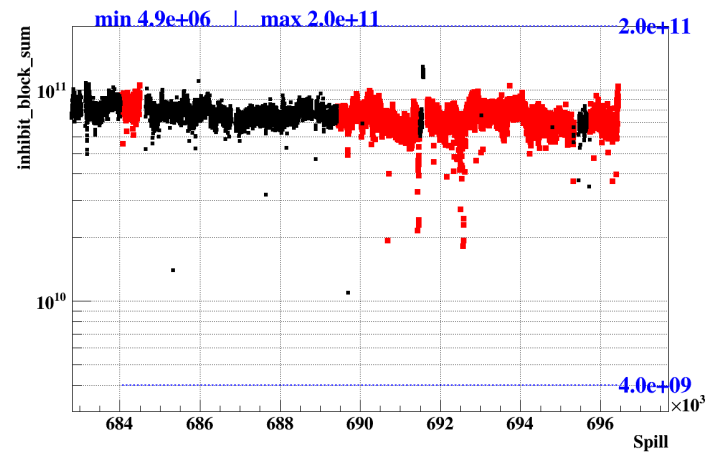

(d) Roadset 70

Figure 4.22: Inhibit as a function of spill ID. Red points are the good spill events and black points are events rejected by the spill-level selections. 


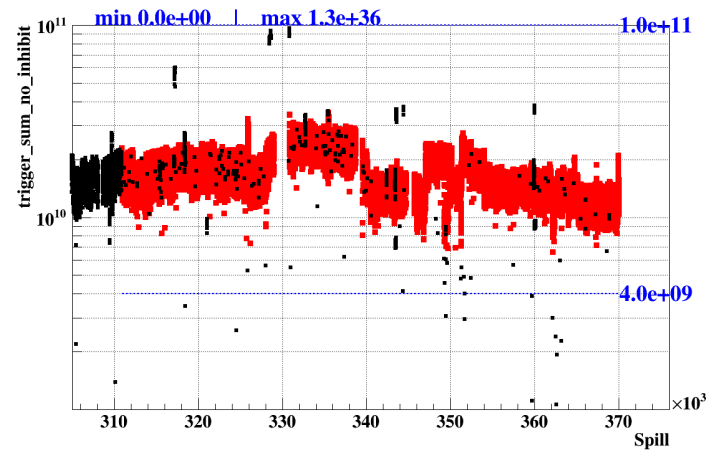

(a) Roadset 57

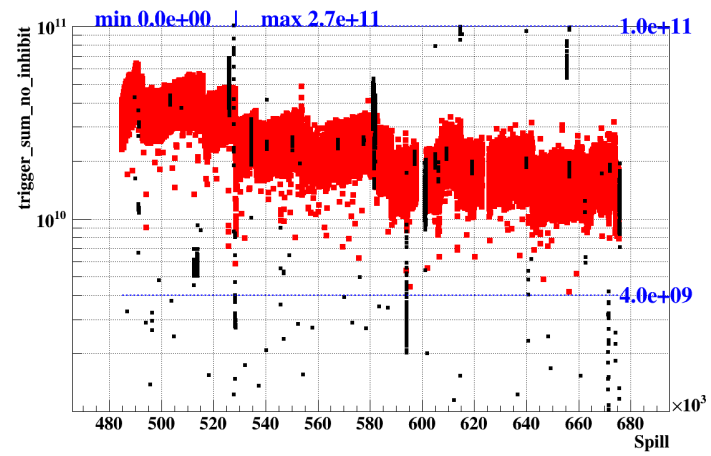

(c) Roadset 67

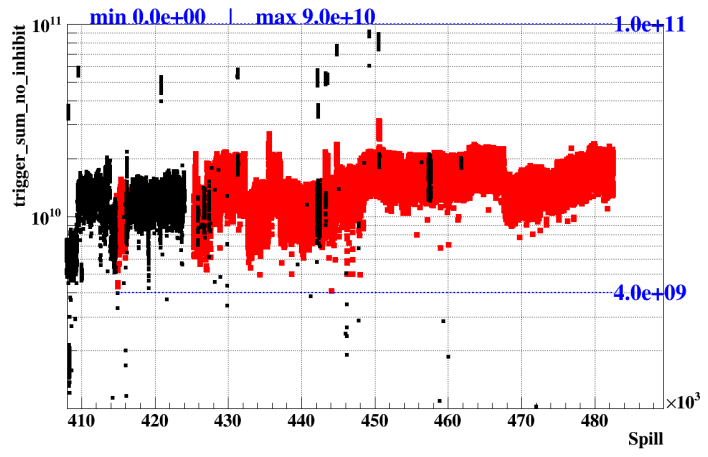

(b) Roadset 62

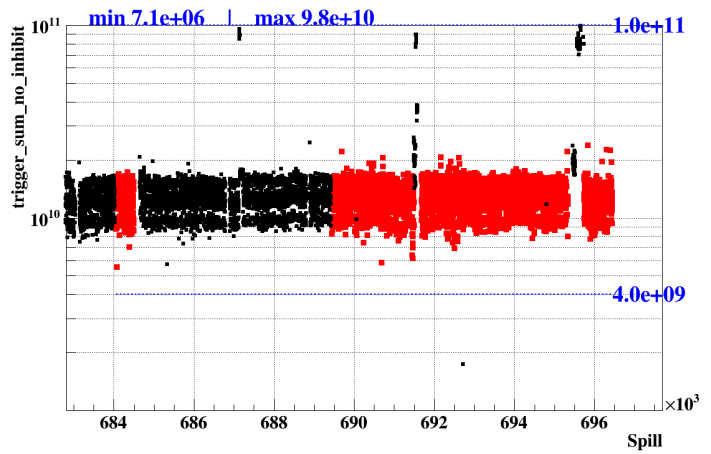

(d) Roadset 70

Figure 4.23: Busy as a function of spill ID. Red points are the good spill events and black points are events rejected by the spill-level selections. 


\subsubsection{Magnets Related Quantities}

"FMag" and "KMag" are the current of FMag and KMag, respectively. If the setting of each magnet is not correct, the current is less than $1000 \mathrm{~mA}$. The selection is made based on this.

\subsubsection{Event Level Selection}

There are two types of event level selections. One is for the track selection, and the other is for the dimuon selection.

\subsubsection{Track Selection}

The track selection is applied to all the reconstructed tracks. The cuts are listed in Tab. 4.6. In addition

Table 4.6: Track selection.

\begin{tabular}{lrr}
\hline Quantity & Min. & Max. \\
\hline Number of track associated hits & 15 & 18 \\
Reduced chi-square & 0.0 & 5.0 \\
$z$-vertex position $(\mathrm{cm})$ & -400.0 & 200.0 \\
$z$-momentum at St. 1 (if \# of hits $<18)$ & 18.0 & - \\
\hline
\end{tabular}

to the list, each track should have a proper road ID. The momentum cut is applied for reducing combinatorial backgrounds.

Figure 4.24, 4.25, 4.26 and 4.27 show the quantities used for the track selections. All the events used for these plots satisfy the spill-level selections.

The track reconstruction tool requires at least 4 hits in a drift chamber since the number of parameters used for the local tracklet fit is four. However, if the number of hits in a drift chamber is four, the number of degrees of freedom becomes zero. In order to obtain the good quality tracks, the tracks should have 15 hits or more.

The targets are located at $z=-155--105 \mathrm{~cm}$, and the dump is located at $z=0-500 \mathrm{~cm}$. The $z$-vertex selection is set to cover all the regions of the target and former half of the dump.

The selection on $z$-momentum at St. 1 is performed in order to suppress the fake tracks. The distribution shape in Fig. 4.27 looks normal. Figure 4.28 is the distribution of $z$-momentum at St. 1 with selections of "Number of track associated hits $=15$ " and the dimuon mass $>4.2 \mathrm{GeV}$. The low $z$-momentum tracks appear in Fig 4.28. They are considered to be the fake tracks. The $z$-momentum selection removes such kind of tracks.

Figure 4.29 and Fig. 4.30 are $z$-vertex position and $z$-momentum at St. 1 of the track of MonteCarlo data for the references. 


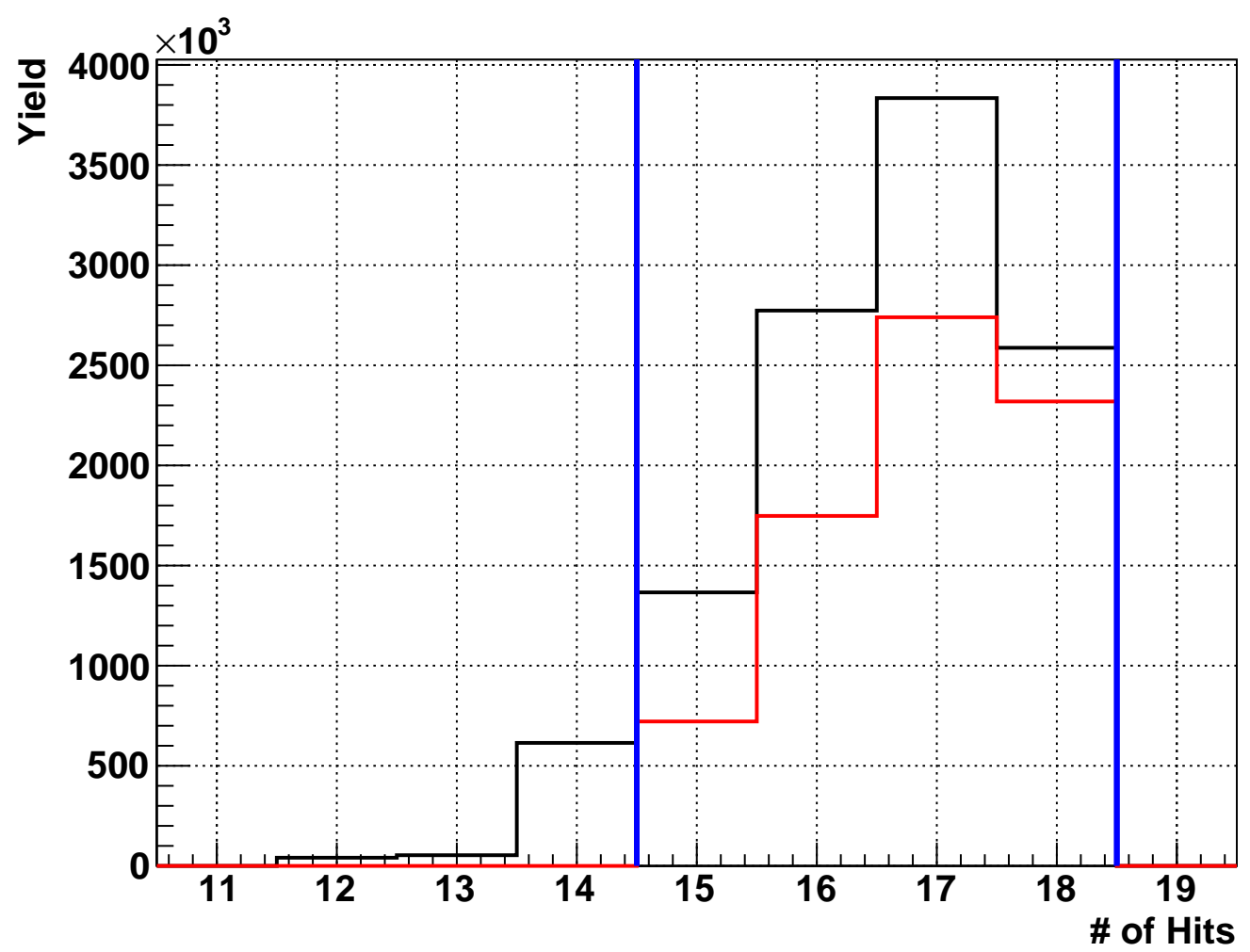

Figure 4.24: Number of track associated hits. The events with black line are all the events. The events drawn with a red line satisfy the track selections. The blue lines show the upper (18) and lower (15) limits of the selection for the number of track associated hits. 


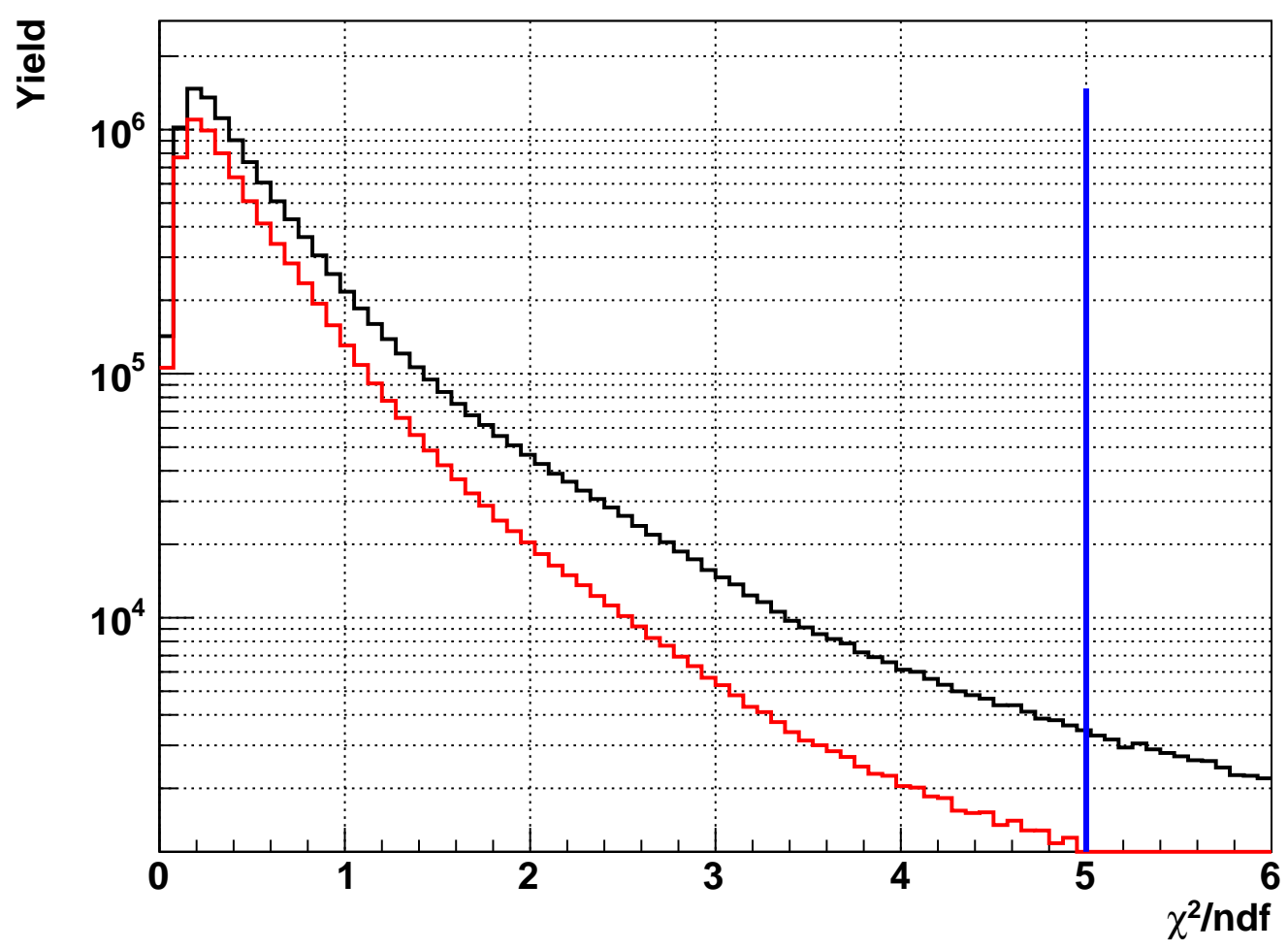

Figure 4.25: Reduced $\chi^{2}$. The events with black line are all the events. The events drawn with a red line satisfy the track selections. The blue line shows the upper limit (5) of the selection for the reduced $\chi^{2}$. 




Figure 4.26: $z$-vertex position. The events with black line are all the events. The events drawn with a red line satisfy the track selections. The blue lines are the upper $(200 \mathrm{~cm})$ and lower $(-400 \mathrm{~cm})$ limits of the selection for the $z$-vertex. 


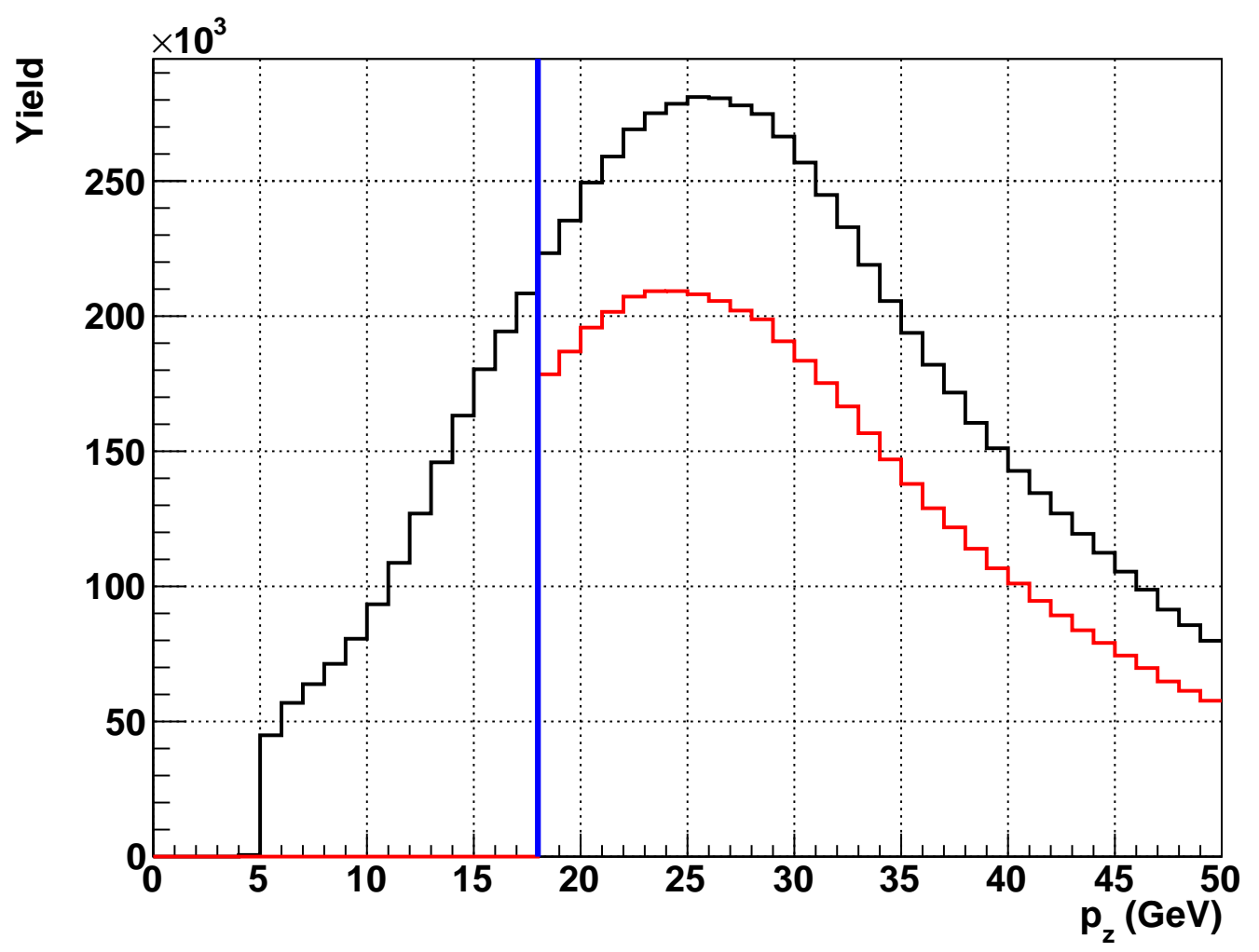

Figure 4.27: $z$-momentum at St. 1. The events with black line are all the events. The events drawn with red line satisfy the track selections. The selection "Number of track associated hits $<18$ " is applied to both black and red lines. The blue line shows the lower limit $(18 \mathrm{GeV})$ of the selection for the $z$-momentum at St. 1. 


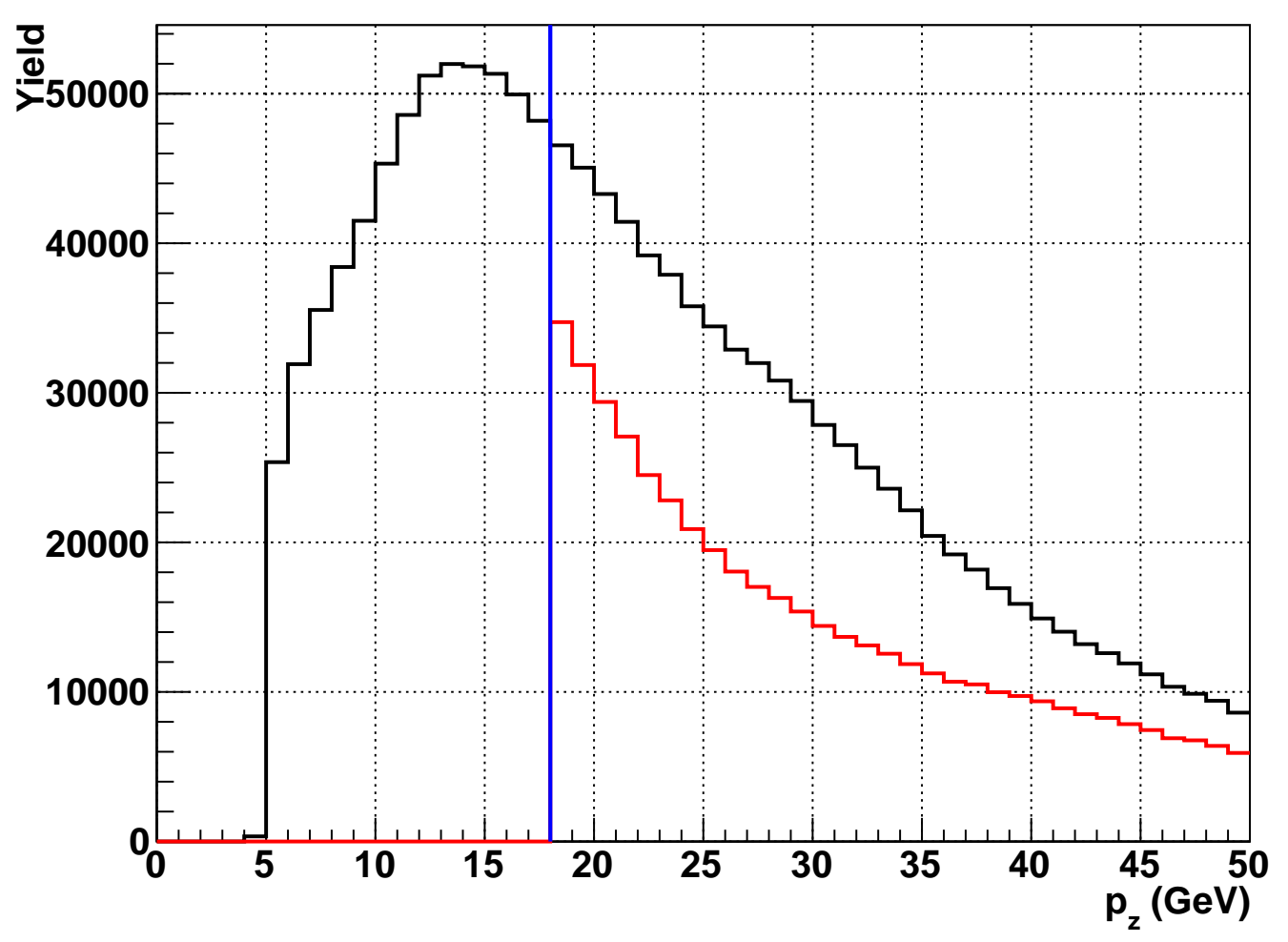

Figure 4.28: $z$-momentum at St. 1 with selections of "Number of track associated hits=15" and dimuon mass $>4.2 \mathrm{GeV}$. The events with black line are all the events. The events drawn with a red line satisfy the track selections. The blue line shows the lower limit $(18 \mathrm{GeV})$ of the selection for the $z$-momentum at St. 1 . 


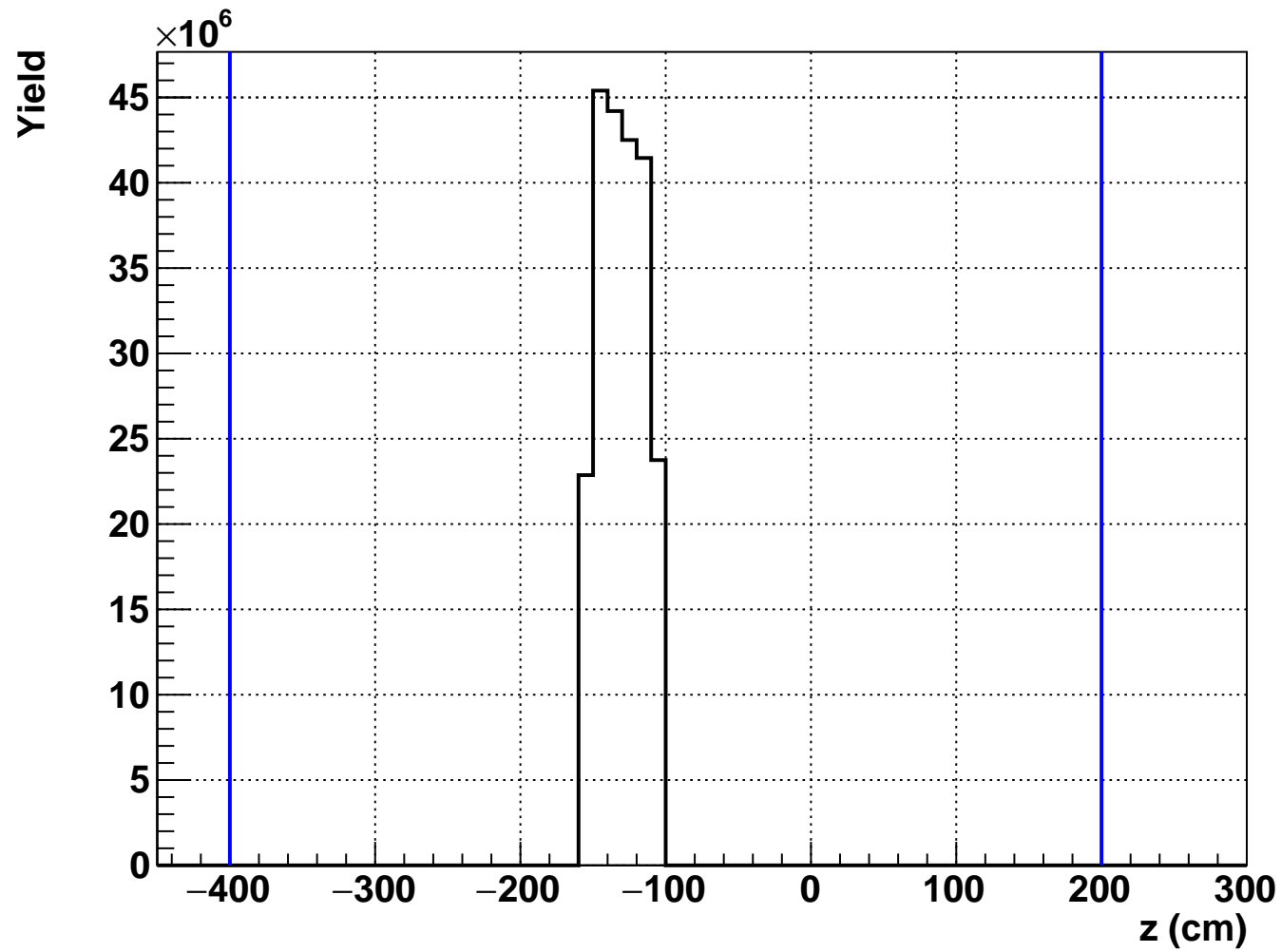

Figure 4.29: $z$-vertex position of Monte-Carlo data. The blue lines show the lower and upper limits of the selection for the $z$-vertex position. 


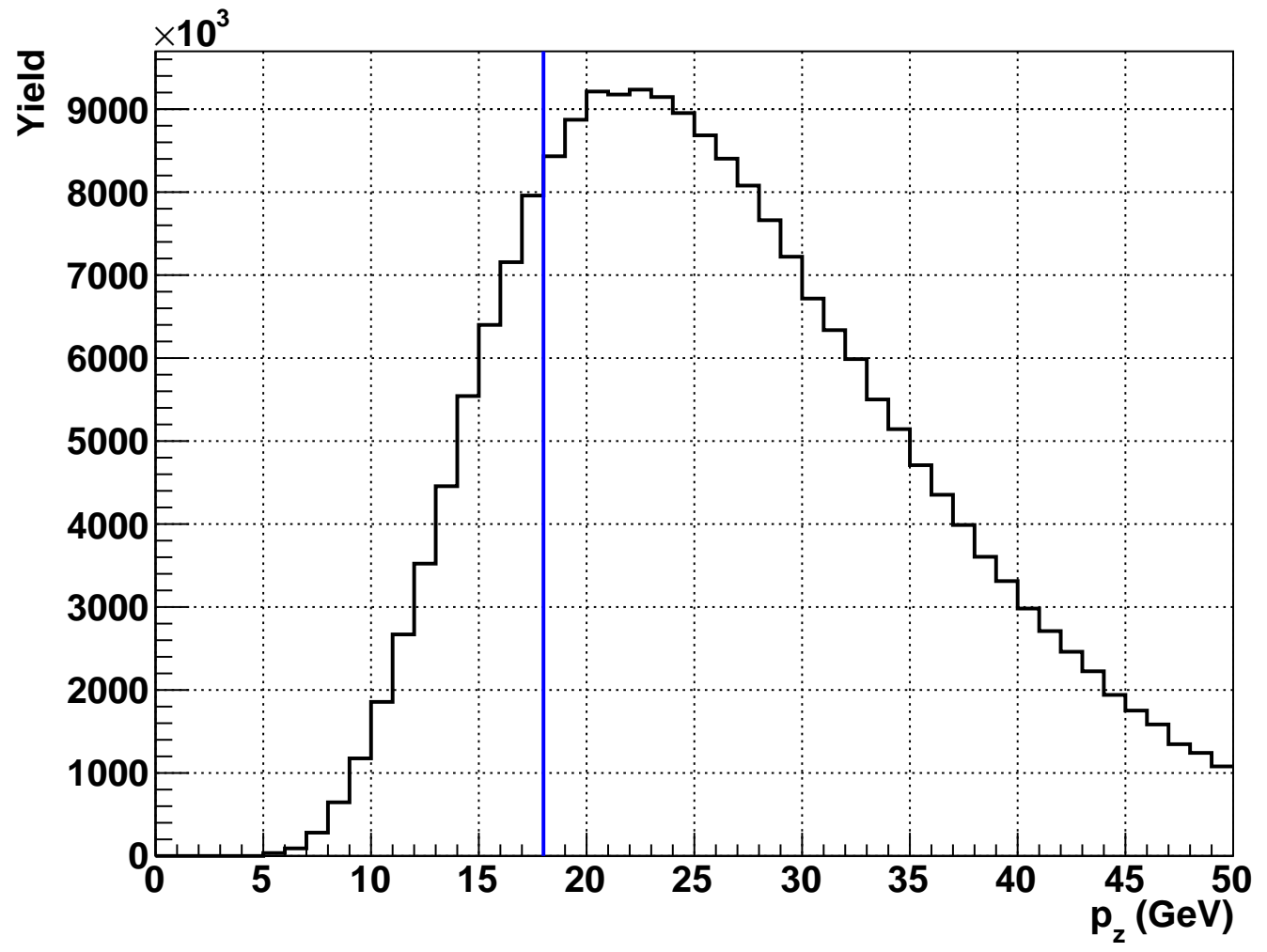

Figure 4.30: $z$-momentum at St. 1 of Monte-Carlo data. The blue line shows the lower limit $(18 \mathrm{GeV})$ of the selection for the $z$-momentum at St. 1 . 


\subsubsection{Dimuon Selection}

The dimuon selection is applied to all the reconstructed dimuons. The cuts are listed in Tab. 4.7. In Table 4.7: Dimuon selection.

\begin{tabular}{lrr}
\hline Quantity & Min. & Max. \\
\hline$x$-vertex position $(\mathrm{cm})$ & -2.0 & 2.0 \\
$y$-vertex position $(\mathrm{cm})$ & -2.0 & 2.0 \\
$z$-vertex position $(\mathrm{cm})$ & -300.0 & 200.0 \\
$x$-momentum at vertex $(\mathrm{GeV} / \mathrm{c})$ & -3.0 & 3.0 \\
$y$-momentum at vertex $(\mathrm{GeV} / \mathrm{c})$ & -3.0 & 3.0 \\
$z$-momentum at vertex $(\mathrm{GeV} / \mathrm{c})$ & 30.0 & 120.0 \\
Bjorken $x$ of beam & 0.0 & 1.0 \\
Bjorken $x$ of target & 0.0 & 1.0 \\
Feynman $x$ & -1.0 & 1.0 \\
Difference between $z$-vertex positions of $\mu^{+}$and $\mu^{-}$ & -250.0 & 250.0 \\
$\chi^{2}$ of vertex reconstruction & 0.0 & 15.0 \\
$x$-momentum of $\mu^{+}$ & 0.0 & - \\
$x$-momentum of $\mu^{-}$ & - & 0.0 \\
mass & 4.2 & - \\
\hline
\end{tabular}

addition to the list, both tracks in a dimuon should satisfy the track selection.

The following figures show the quantities used for the dimuon selections. All the events satisfy the spill and track selections.

The $x$ and $y$ values of the vertex position should be close to zero. The $z$-vertex position range is from the target position to the dump position. The separation of target and dump events is applied after the dimuon selections. The vertex position distributions with Monte-Carlo are shown in Fig. 4.32 for the references. The momentum selections at the vertex is also performed. Since the SeaQuest

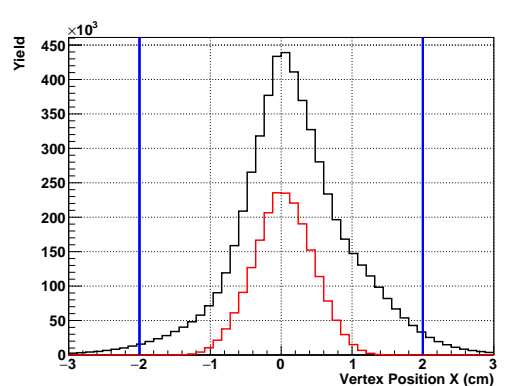

(a) $x$



(b) $y$

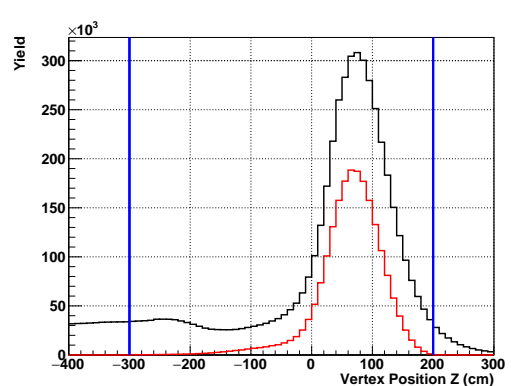

(c) $z$

Figure 4.31: Vertex position. The distributions of $x, y$ and $z$ are shown in (a), (b) and (c), respectively. The events with black line are all the events. The events drawn with a red line satisfy the dimuon selections. The blue lines show the upper and lower limits of the selection for the vertex position.

spectrometer is located in the forward direction of the target, the high- $p_{T}$ events are not detected. 


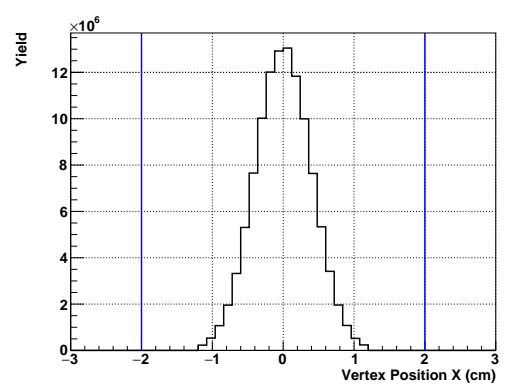

(a) $x$

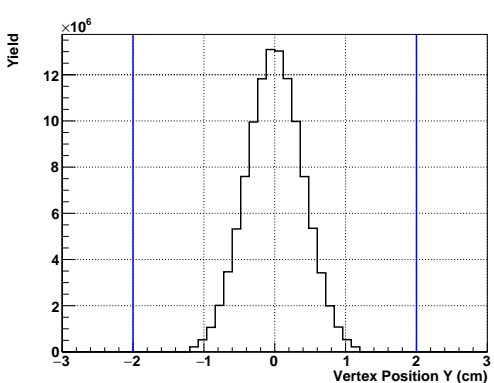

(b) $y$

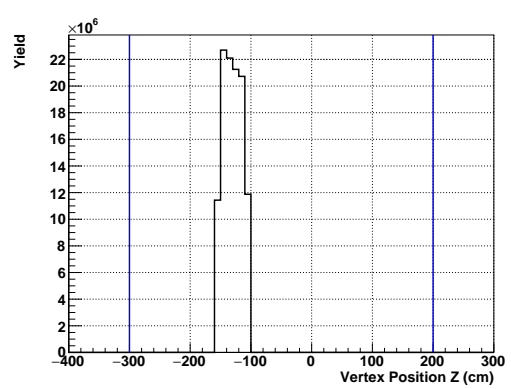

(c) $z$

Figure 4.32: Vertex position of Monte-Carlo data.

Therefore, the $x$ and $y$ momentum should be close to zero. The $z$-momentum should be less than 120 $\mathrm{GeV} / \mathrm{c}$ since the beam energy is $120 \mathrm{GeV}$. The lower limit of the $z$-momentum is determined by the Mote-Carlo as shown in Fig. 4.34. The vertex momentum distributions with Monte-Carlo are shown in Fig. 4.34 for the references.

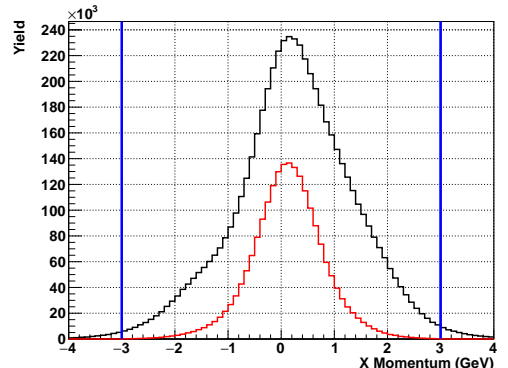

(a) $x$

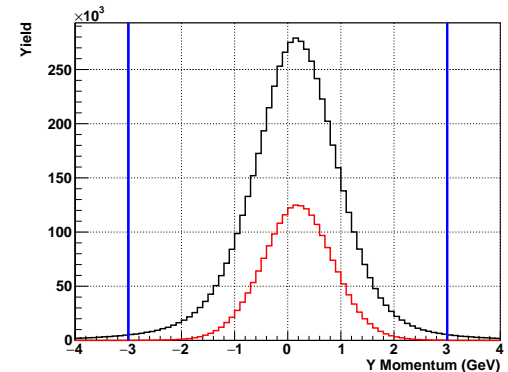

(b) $y$

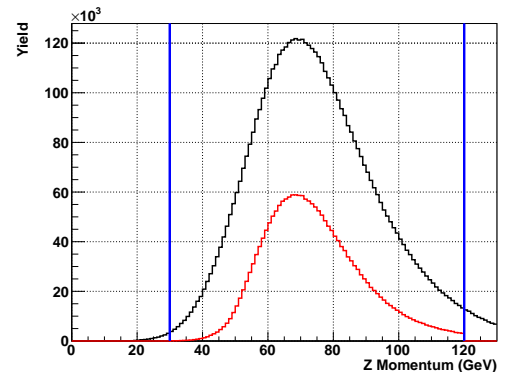

(c) $z$

Figure 4.33: Momentum at vertex position. The components of $x, y$ and $z$ are shown in (a), (b) and (c), respectively. The events with black line are all the events. The events drawn with a red line satisfy the dimuon selections. The blue lines show the upper and lower limits of the selection for the vertex position.



(a) $x$

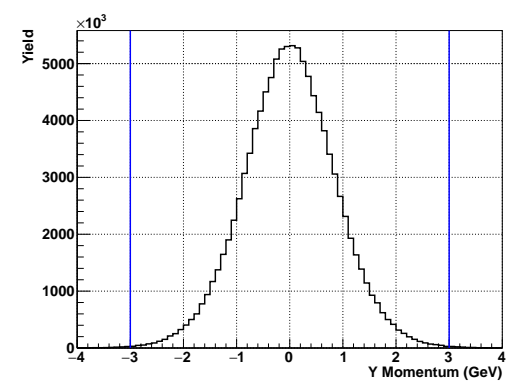

(b) $y$

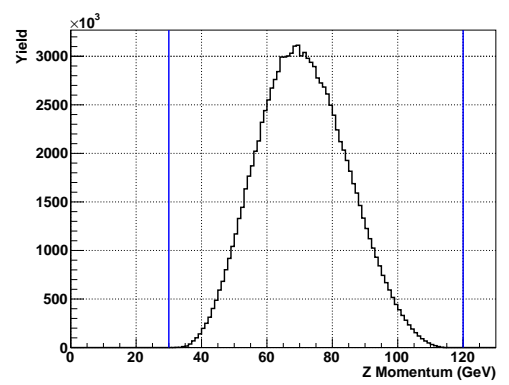

(c) $z$

Figure 4.34: Momentum at vertex position of Monte-Carlo data.

The Bjorken $x$ range should be $0<x<1$, and $x_{F}$ range should be $-1<x_{F}<1$. Figure 4.35 and Fig. 4.37 show the $x_{1}, x_{2}$ and $x_{F}$ distributions. Figure 4.36 and Fig. 4.38 are the $x_{1}, x_{2}$ and $x_{F}$ distributions with Monte-Carlo for the references.

The difference between $z$-vertex positions of $\mu^{+}$and $\mu^{-}$, namely track separation, indicates the 


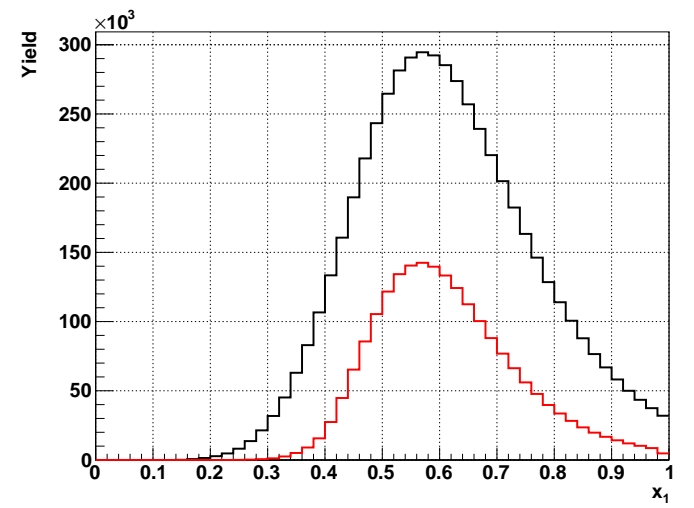

(a) $x_{1}$

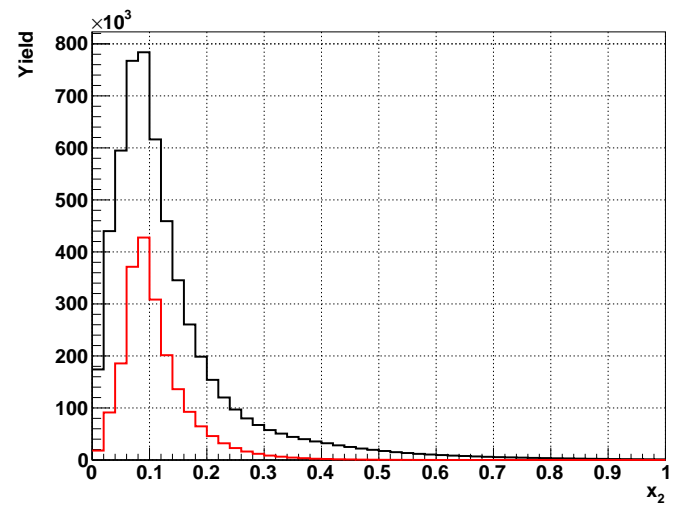

(b) $x_{2}$

Figure 4.35: Bjorken $x$ of beam $\left(x_{1}\right.$, (a)) and target $\left(x_{2}\right.$, (b)) of the read data. The events with black line are all the events. The events drawn with a red line satisfy the dimuon selections.

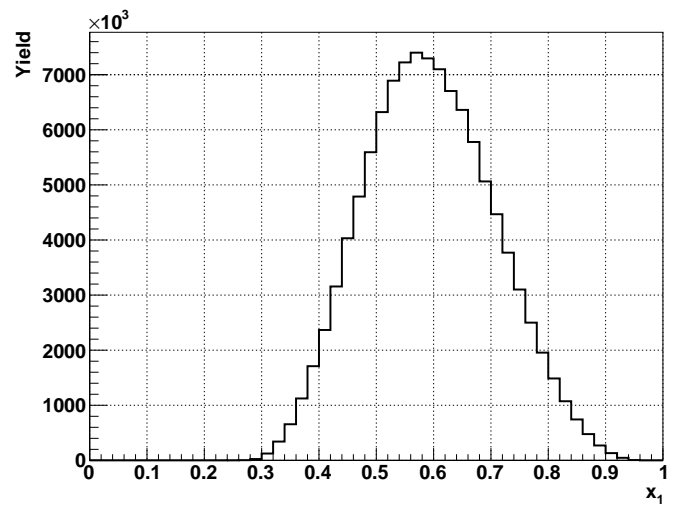

(a) $x_{1}$

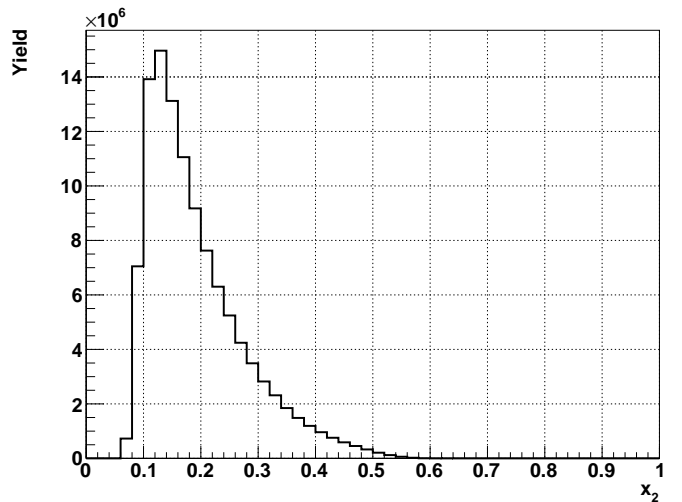

(b) $x_{2}$

Figure 4.36: Bjorken $x$ of beam $\left(x_{1}\right.$, (a)) and target $\left(x_{2},(\mathrm{~b})\right)$ of Monte-Carlo data. 


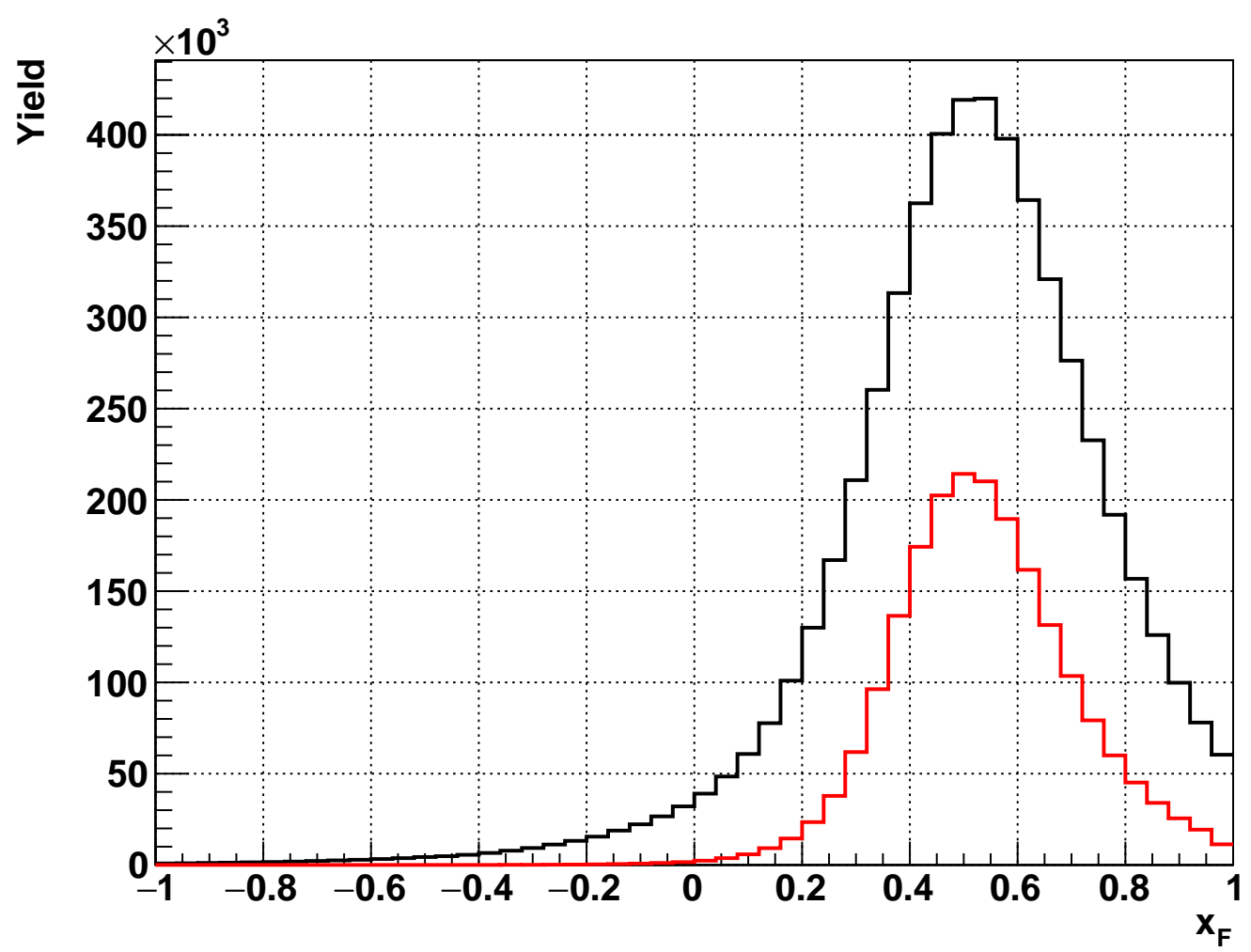

Figure 4.37: Feynman $x$ distributions of the real data. The events with black line are all the events. The events drawn with a red line satisfy the dimuon selections.

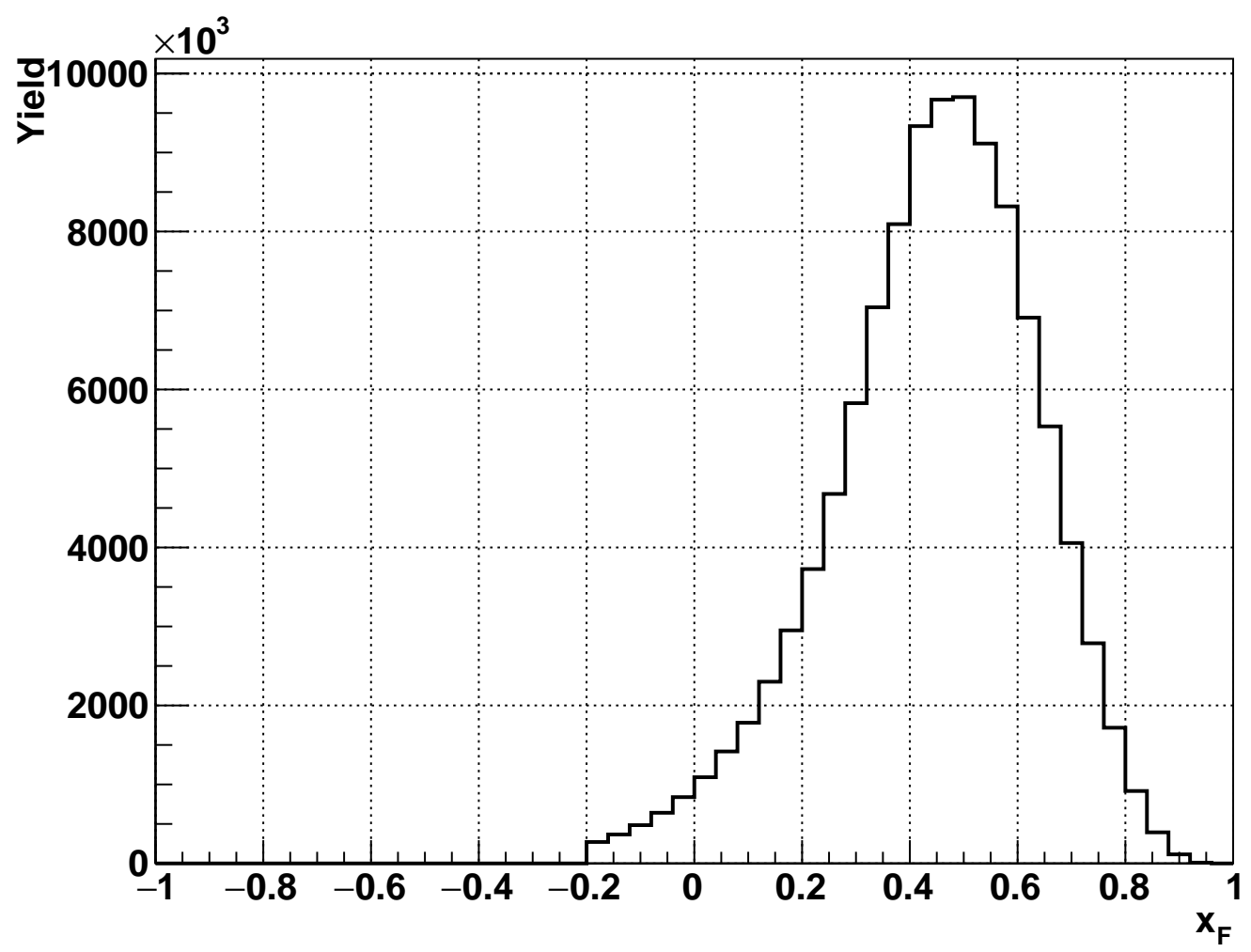

Figure 4.38: Feynman $x$ of Monte-Carlo data. 
probability that the two tracks construct a dimuon. The $z$-vertex positions of the two tracks originating from a dimuon should be close each other.

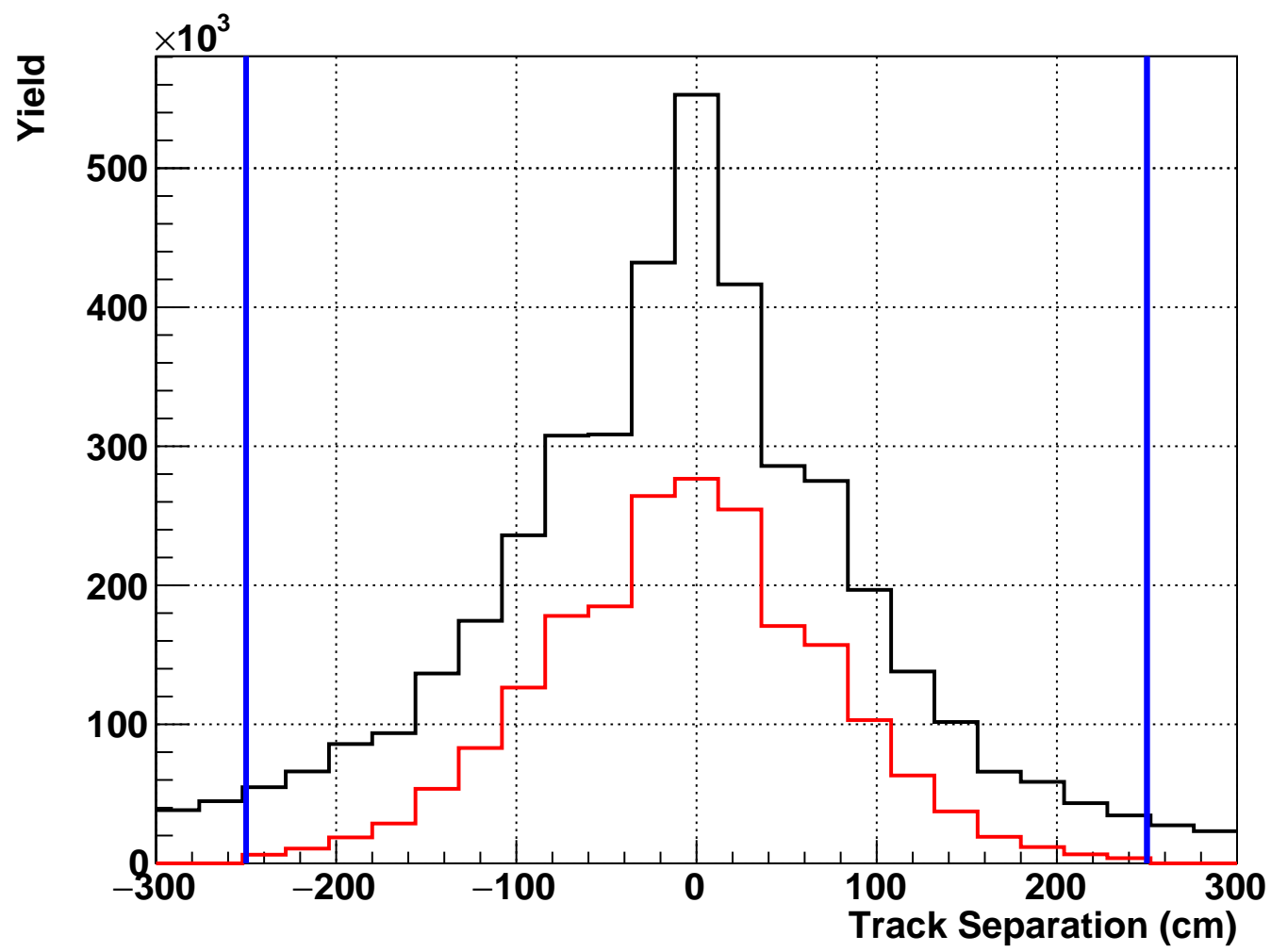

Figure 4.39: Difference between $z$-vertex positions of $\mu^{+}$and $\mu^{-}$. The events with black line are all the events. The events drawn with a red line satisfy the dimuon selections. The blue lines show the upper and lower limits of the selection for the difference between $z$-vertex positions.

The $\chi^{2}$ of vertex reconstruction indicates how close the distance between the track in a dimuon and beam line at the vertex position is. The dimuon quality is better if the $\chi^{2}$ is closer to zero.

The $x$-momentum of $\mu^{+}$at St. 1 should be positive when the magnet polarity is up (Roadset 57 and 62), and should be negative when the magnet polarity is down (Roadset 67 and 70). The data set used in Fig. 4.41 is Roadset 57, thus the $x$-momentum of $\mu^{+}$should be positive and that of $\mu^{-}$ should be negative. Figure 4.42 is the plot of the same quantity but with the Monte-Carlo data for the references.

Figure 4.43 shows the dimuon mass distribution with component fitting. The fitting is performed with the Drell-Yan, $J / \psi, \psi^{\prime}$ Monte-Carlo and the random background estimated with real data. The event mixing method is used for the random background estimation. The procedure of the event mixing method is:

- Take a single positive track from an FPGA-1 triggered event with only one reconstructed track.

- Take another single negative track from another FPGA-1 triggered event with only one reconstructed track.

- Reconstruct a dimuon from the two track. 


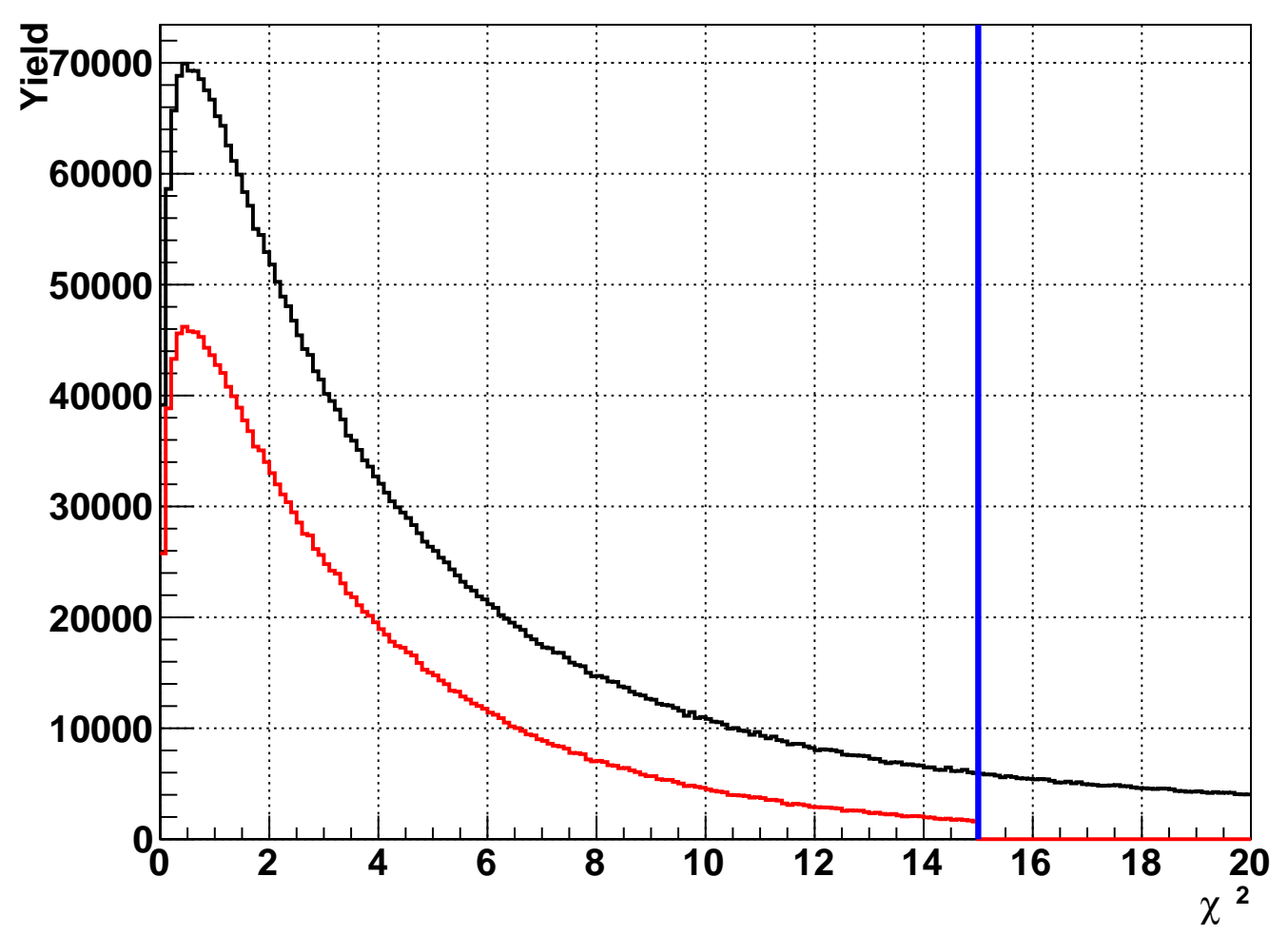

Figure 4.40: $\chi^{2}$ of vertex reconstruction. The events with black line are all the events. The events drawn with a red line satisfy the dimuon selections. The blue line shows the upper limit of the selection for the $\chi^{2}$.

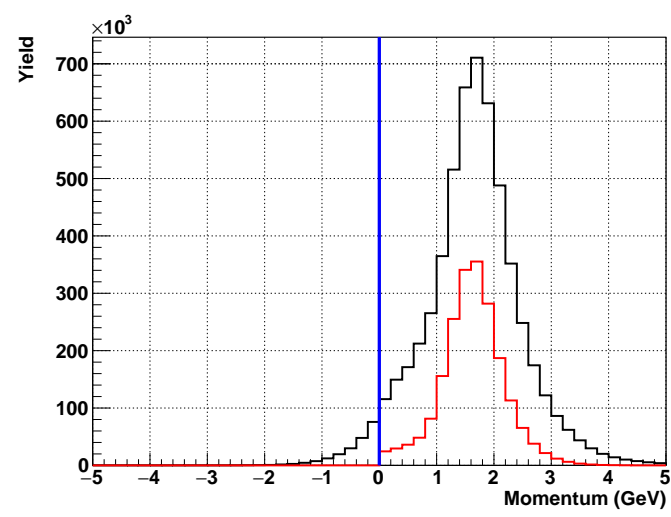

(a) $\mu^{+}$

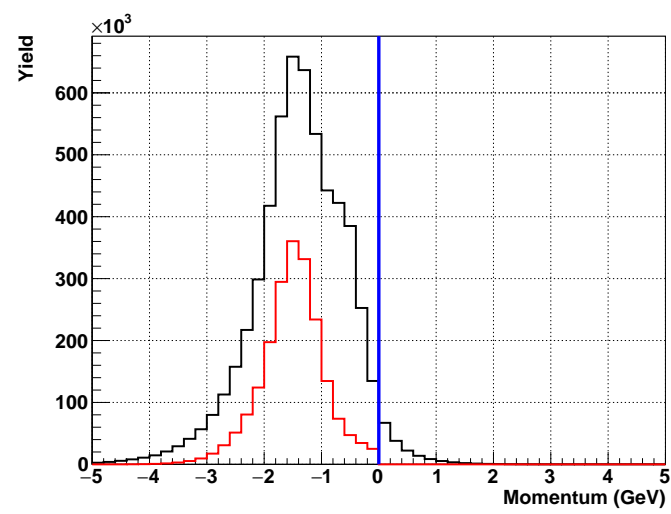

(b) $\mu^{-}$

Figure 4.41: $x$-momentum of $\mu^{+}$and $\mu^{-}$. The events with black line are all the events. The events drawn with a red line satisfy the dimuon selections. The blue lines show the upper and lower limits of the selection for the $x$-momenta. 


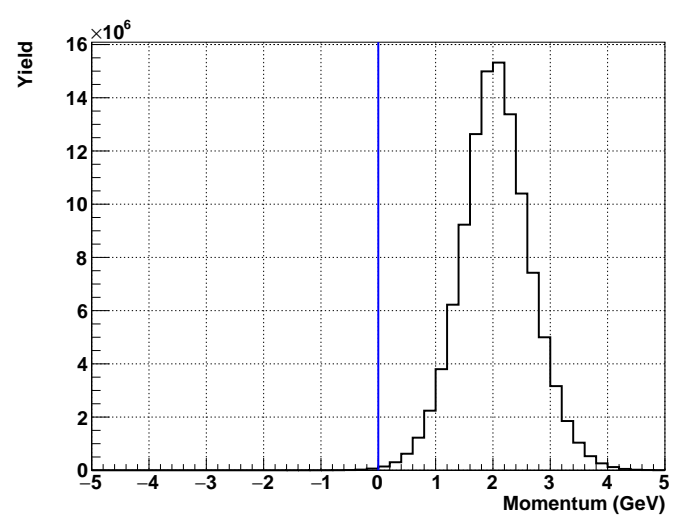

(a) $\mu^{+}$

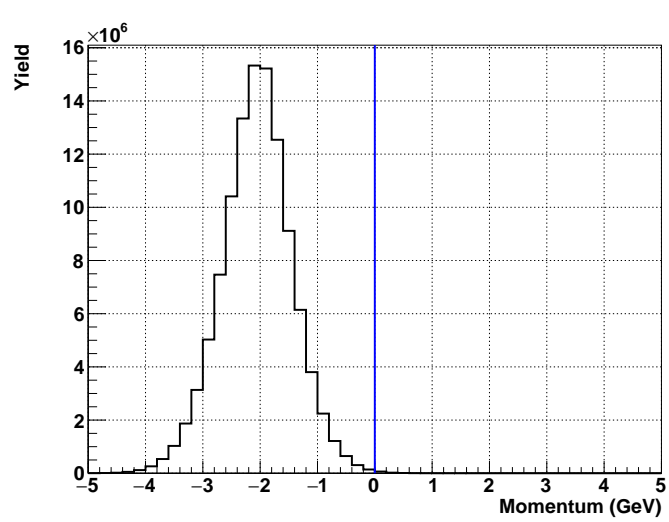

(b) $\mu^{-}$

Figure 4.42: $x$-momentum of $\mu^{+}$and $\mu^{-}$of Monte-Carlo data.

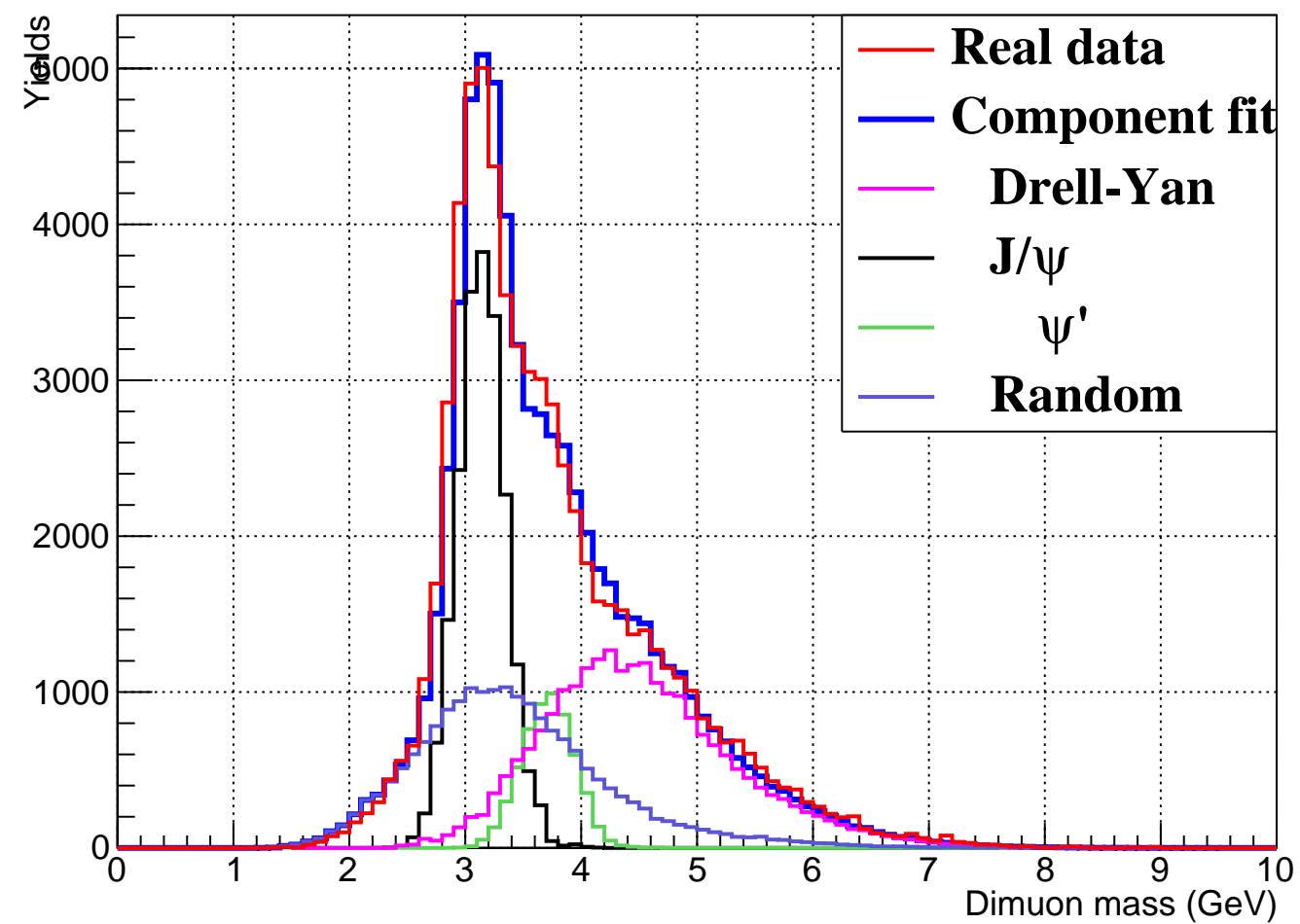

Figure 4.43: Dimuon mass. The red line is real data, the purple line is Drell-Yan Monte-Carlo, the green lines are $J / \psi$ and $\psi^{\prime}$ Monte-Carlo, the light blue line is the random background estimated with real data using event mixing, and the blue line is sum of Monte-Carlo and random background. 
The Drell-Yan dimuons are dominant at mass $>4.2 \mathrm{GeV}$ beyond the random background. The dimuon mass $>4.2 \mathrm{GeV}$ selection is applied to use data in the Drell-Yan dominant region.

\subsubsection{Target-Dump Separation}

The dimuons originating from the beam dump are included in the selected dimuons. The target-dump separation is applied in order to use the dimuons originating from target. The target-dump selections are listed in Tab. 4.8 .

Table 4.8: Target-Dump Separation

\begin{tabular}{lrr}
\hline Quantity & Min. & Max. \\
\hline$\chi_{\text {dump }}^{2}-\chi_{\text {target }}^{2}$ of each $\mu^{+}$and $\mu^{-}$ & 10.0 & - \\
$z$-vertex position $(\mathrm{cm})$ & -300 & -60 \\
\hline
\end{tabular}

$\chi_{\text {dump }}^{2}$ and $\chi_{\text {target }}^{2}$

The quantities $\chi_{\text {dump }}^{2}$ and $\chi_{\text {target }}^{2}$ are used for the target and dump separation. The $\chi_{\text {target }}^{2}\left(\chi_{\text {dump }}^{2}\right)$ indicates how close to the target (dump) position from the single track. Therefore, this selection is applied in track-level. Figure 4.44 and Fig. 4.45 show the distributions of $\chi_{\text {target }}^{2}$ and $\chi_{\text {dump. }}^{2}$ Using these two variables, the selection of $\chi_{\text {dump }}^{2}-\chi_{\text {target }}^{2}$ is applied for the target dimuons. Figure 4.46 shows the distribution of $\chi_{\text {dump }}^{2}-\chi_{\text {target }}^{2}$. There is a hollow at $\chi_{\text {dump }}^{2}-\chi_{\text {target }}^{2} \sim 10$. The events with $\chi_{\text {dump }}^{2}-\chi_{\text {target }}^{2}>10$ comes from the target.

\section{$z$-vertex Position}

The $z$-vertex position selection is needed again because the $z$-vertex selections in track and dimuon selections accept the dump events. Figure 4.47 shows the $z$-vertex position distribution. The black line shows all the events which satisfy the spill-, track-, and dimuon-selections. The red line shows the events which is subset of the black line with passing the target selection. The blue lines shows the lower and upper limits of the $z$-vertex position selection. The red line is well separated from dump. The additional proof that the target events and dump events are well separated is shown in Fig. 4.48. The circles show z-vertex distribution of the muon pairs in real data. The solid lines are estimated $z$-vertex distributions of muon pairs from target (red line) and that of muon pairs from dump (blue line) in the simulation. The data and simulation are consistent and the target and dump separation works well. 


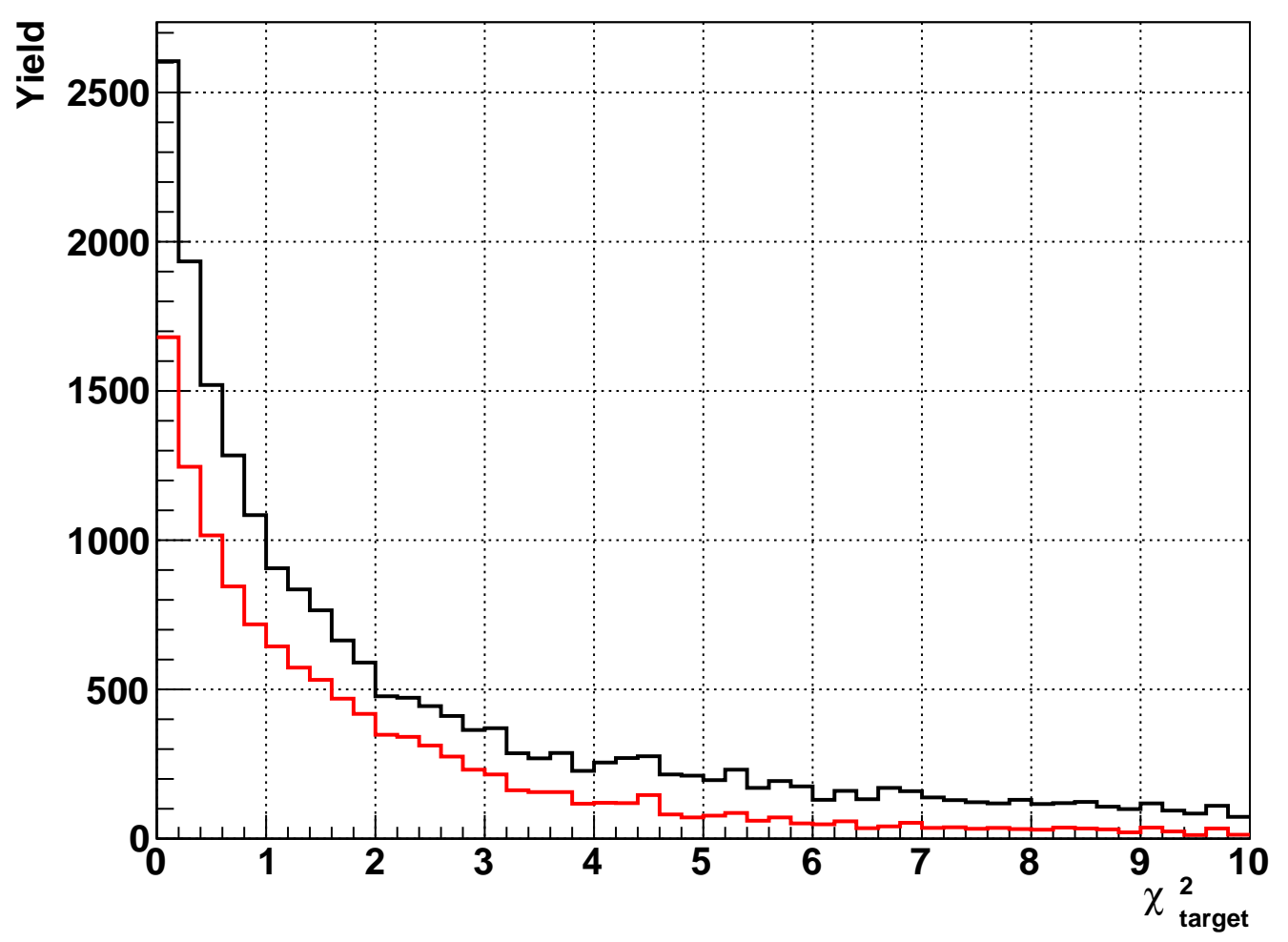

Figure 4.44: $\chi_{\text {target }}^{2}$ of $\mu^{+}$and $\mu^{-}$. The black line shows all the events which satisfy the spill-, track-, and dimuon-selections. The red line shows the events which is subset of the black line with passing the target selection. 


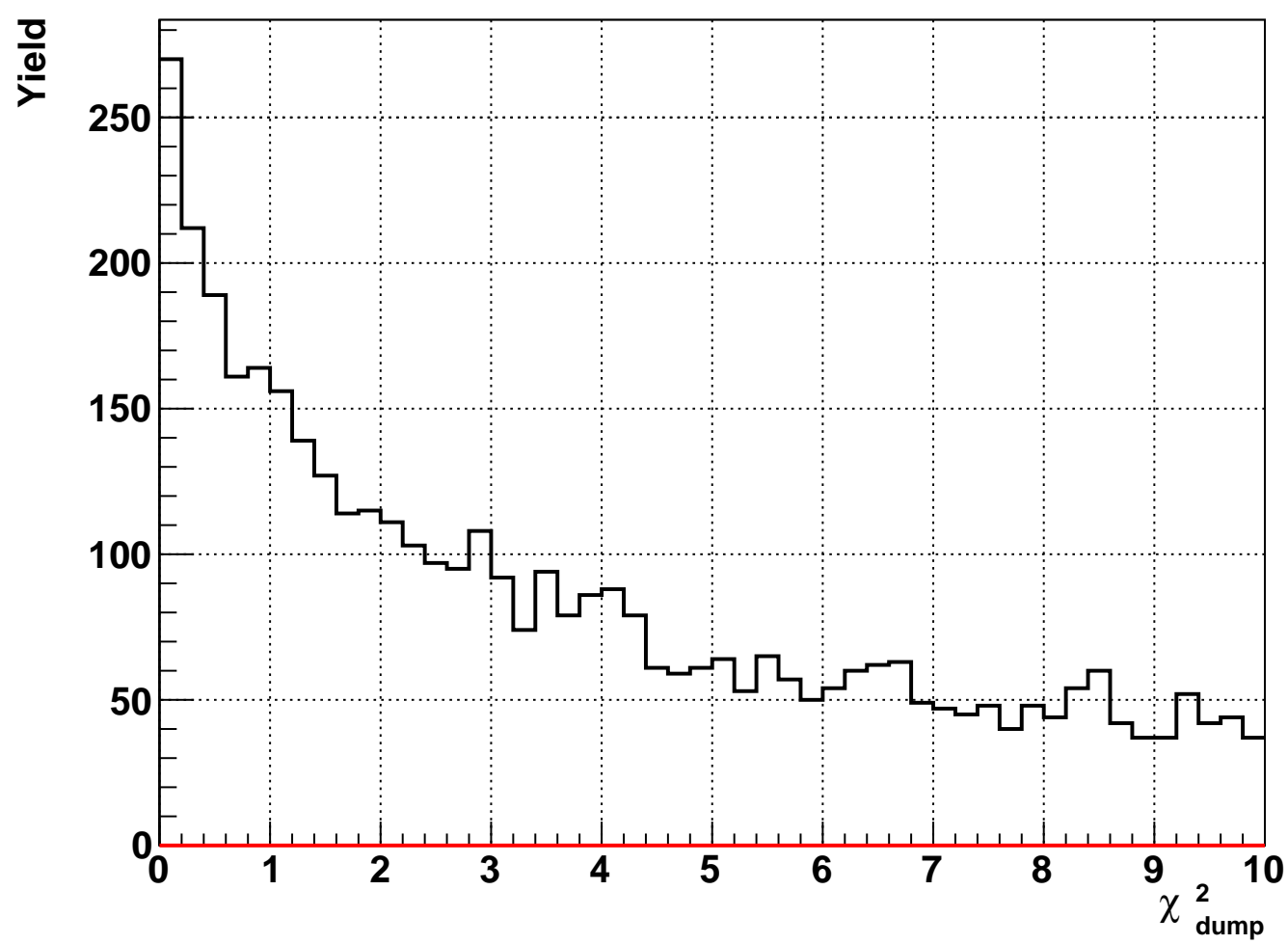

Figure 4.45: $\chi_{\text {dump }}^{2}$ of $\mu^{+}$and $\mu^{-}$. The black line shows all the events which satisfy the spill-, track-, and dimuon-selections. No events pass the target selection. 


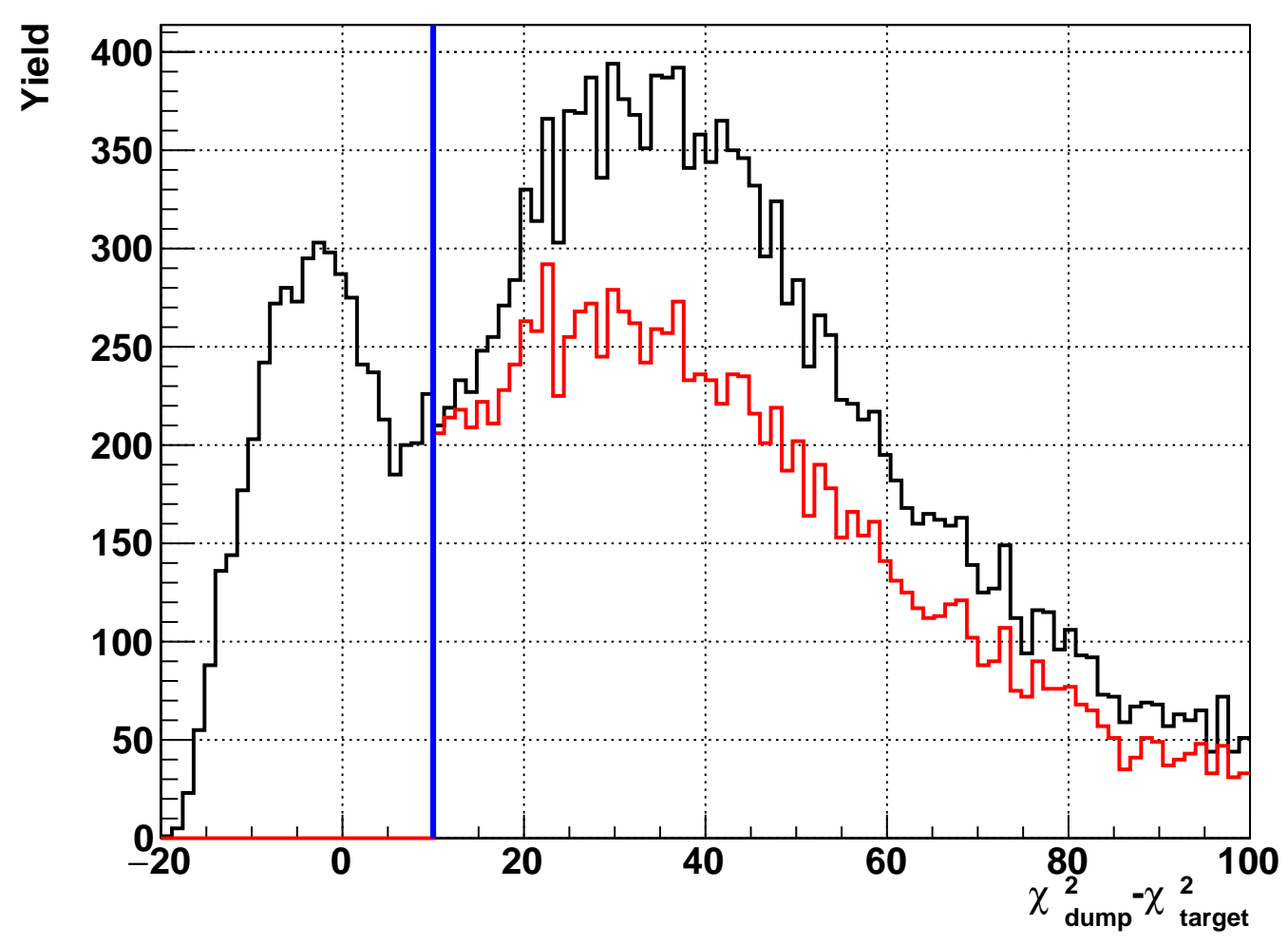

Figure 4.46: $\chi_{\text {dump }}^{2}-\chi_{\text {target }}^{2}$ of $\mu^{+}$and $\mu^{-}$. The black line shows all the events which satisfy the spill-, track-, and dimuon-selections. The red line shows the events which is subset of the black line with passing the target selection. The blue line shows the lower limit (10) of the target selection. 


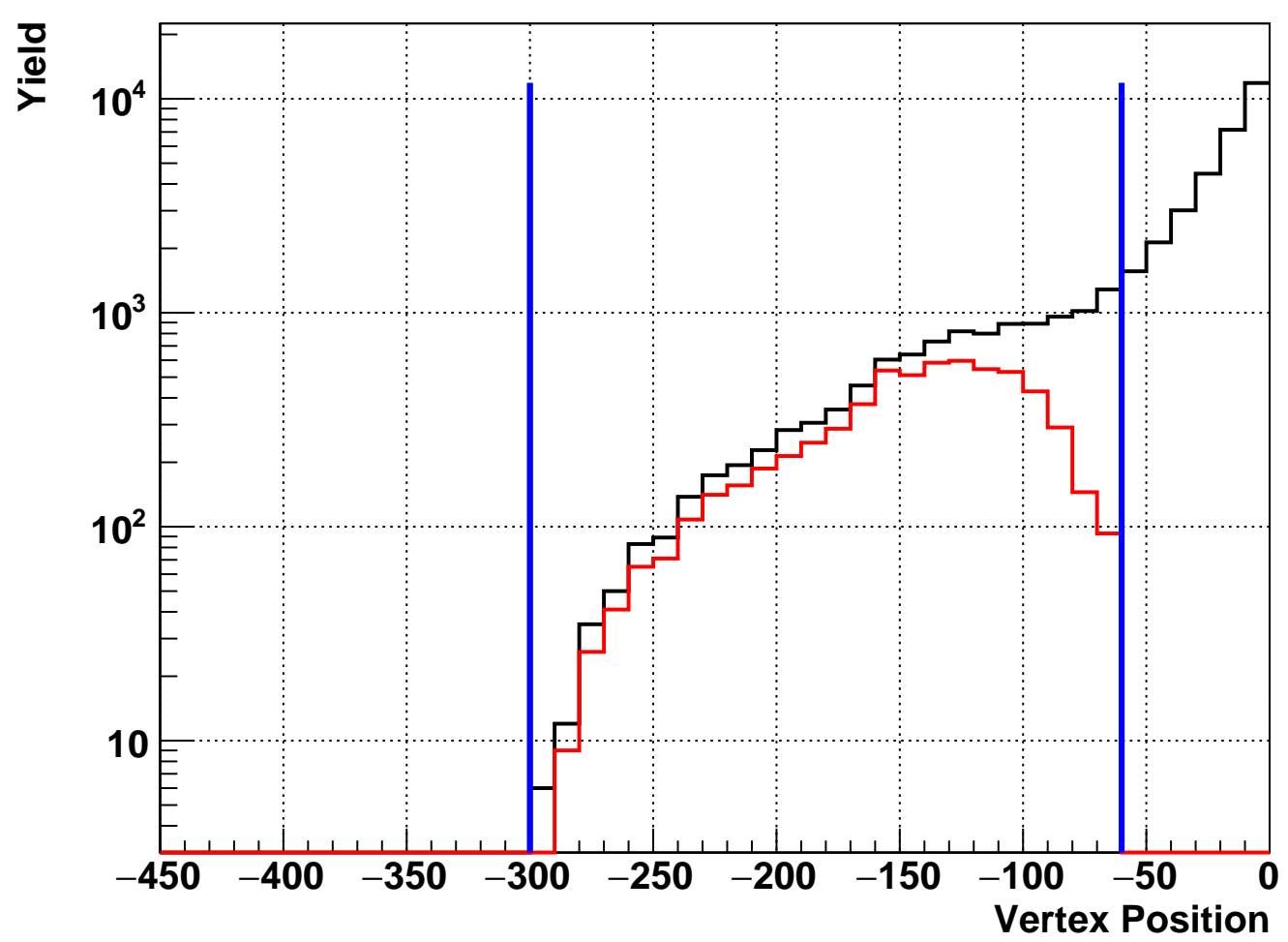

Figure 4.47: $z$-vertex position The black line shows all the events which satisfy the spill-, track-, and dimuonselections. The red line shows the events which is subset of the black line with passing the target selection. The blue lines shows the lower and upper limits of the $z$-vertex position selection. 


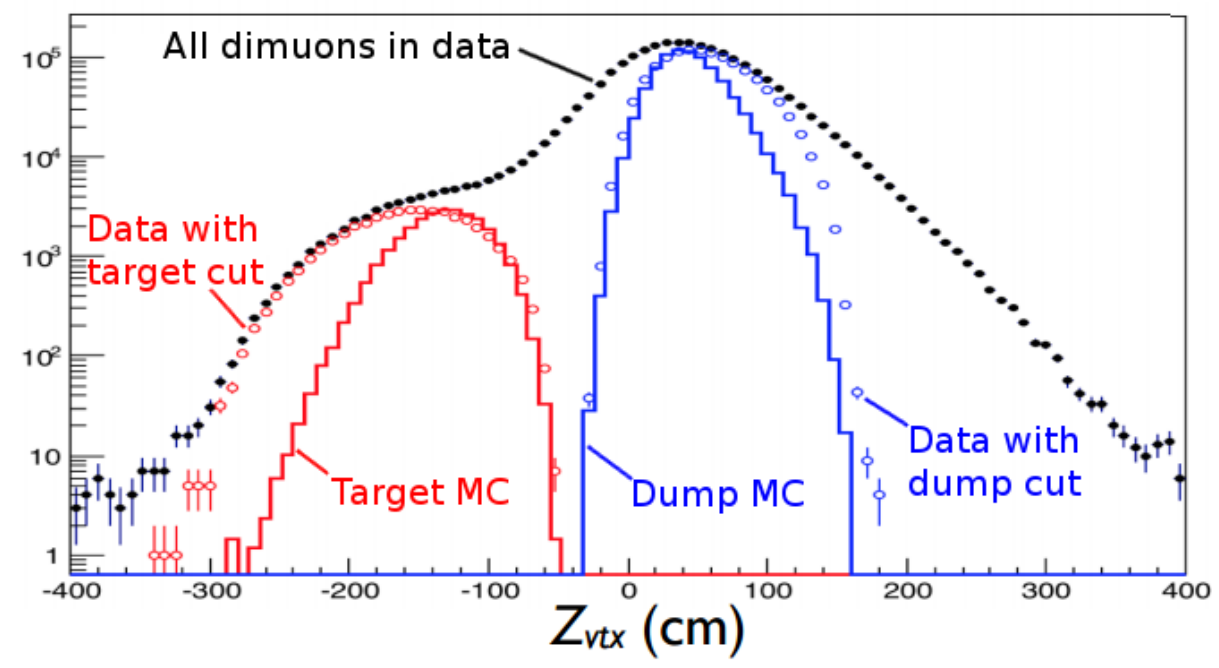

Figure 4.48: $z$-vertex distribution of muon pairs whose invariant masses are larger than $4.2 \mathrm{GeV} / c^{2}$ [43]. The circles show $z$-vertex distribution of the muon pairs in real data. The solid lines are estimated $z$-vertex distributions of muon pairs from target (red line) and that of muon pairs from dump (blue line) in the simulation. 


\subsection{Dimuon Yield}

Using all the selections mentioned above, the dimuon yields per $x_{2}$ bin are counted. These numbers are used for extracting the cross-section ratio and $\bar{d} / \bar{u}$. The dimuon yields without any corrections are called "raw yields", and those with corrections are called "corrected yields".

\subsubsection{Dimuon Raw Yield}

The dimuon raw yields per $x_{2}$ bin are summarized in Tab. 4.9. The data in Raodset 57, 62, 67 and 70 are used.

Table 4.9: Dimuon raw yields of $\mathrm{LH}_{2}, \mathrm{LD}_{2}$ and Empty targets.

\begin{tabular}{ccrrr}
\hline$x_{2} \min$ & $x_{2} \max$ & $\mathrm{LH}_{2}$ & \multicolumn{1}{c}{$\mathrm{LD}_{2}$} & Empty \\
\hline 0.10 & 0.13 & 1489 & 1722 & 66 \\
0.13 & 0.16 & 4039 & 4564 & 167 \\
0.16 & 0.20 & 4875 & 5627 & 200 \\
0.20 & 0.24 & 3896 & 4266 & 154 \\
0.24 & 0.29 & 2087 & 2335 & 91 \\
0.29 & 0.35 & 1063 & 1177 & 44 \\
0.35 & 0.45 & 418 & 515 & 24 \\
0.45 & 0.58 & 82 & 78 & 1 \\
\hline Sum & & 17949 & 20284 & 747 \\
\hline
\end{tabular}




\subsubsection{Dimuon Corrected Yield}

As mentioned in Section 4.2, the track reconstruction is affected by the occupancy which is the number of hits in a drift chamber per event. The dimuon raw yield includes not only the Drell-Yan dimuon originating from the $\mathrm{LH}_{2}$ or $\mathrm{LD}_{2}$ but also the dimuons originating from empty flask and combinatorial background. In order to obtain the correct dimuon yield, correction based on the occupancy and empty target subtraction are performed.

Figure 4.49 shows the flow chart of obtaining the dimuon corrected yields.

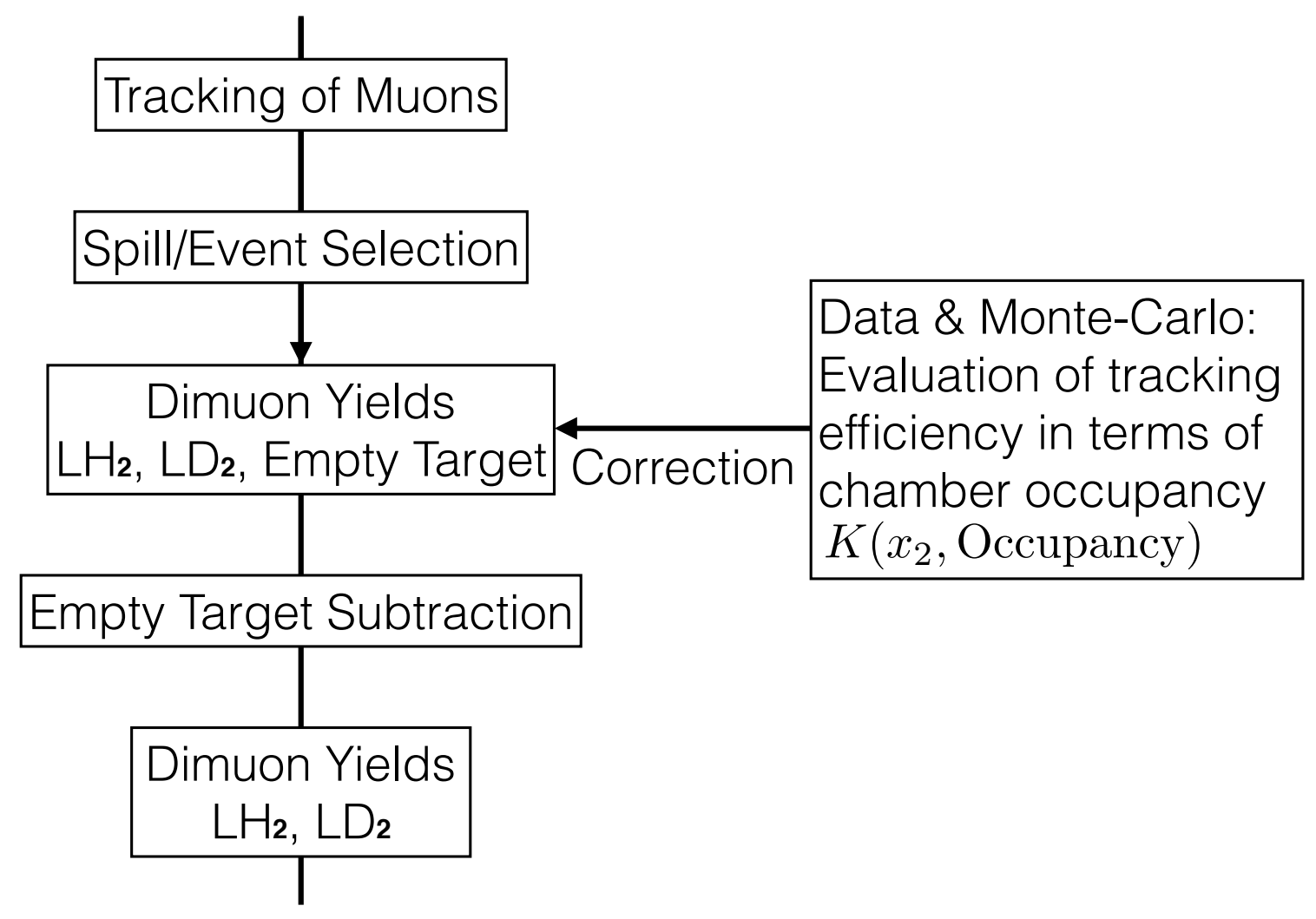

Figure 4.49: Flow chart of obtaining the dimuon corrected yields. 


\subsubsection{Tracking Efficiency Correction}

The effect of the occupancy on the tracking efficiency was estimated with the ratio of the number of "messy GMC" dimuons to that of "clean GMC" dimuons as a function of the occupancy. The "clean GMC" is simply GMC Drell-Yan data. The "messy GMC" data are the mixture of "clean GMC" and random triggered real events. The messy GMC data simulates the real data.

The occupancy in this section is without hit removals mentioned in 4.2.1.1. There are three types of occupancy: occupancy of St. 1 drift chamber, St. 2 drift chamber, and St. 3 drift chamber. Figure 4.50 shows the tracking efficiency as a function of each of St. 1, 2 and 3 occupancy. Each occu-

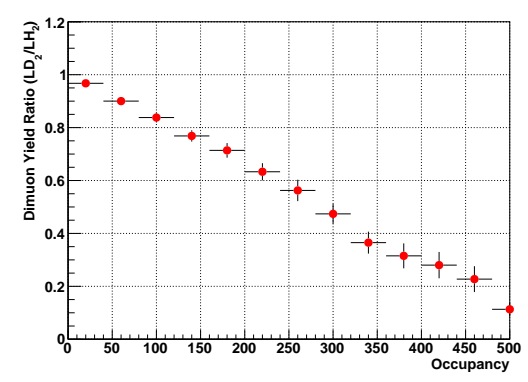

(a) St. 1 occupancy

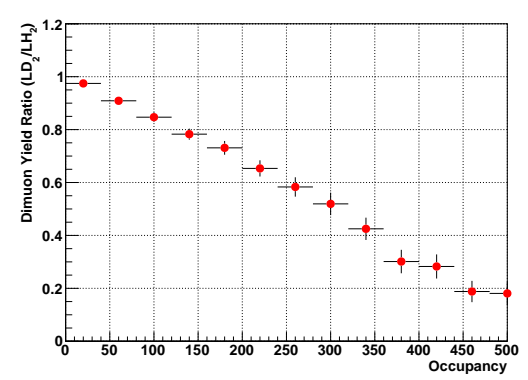

(b) St. 2 occupancy



(c) St. 3 occupancy

Figure 4.50: Tracking efficiency as a function of occupancy. They are the ratio of clean MC and messy MC as a function of (a) St. 1 occupancy, (b) St. 2 occupancy, and (c) St. 3 occupancy.

pancy is well correlated to the tracking efficiency. Therefore, any occupancy can be used to estimate the occupancy dependence. The occupancy of the St. 1 drift chamber should affect the tracking efficiency the most because the hit rate of the St. 1 drift chamber is the highest among those of the drift chambers due to the location of the chamber. In this section, the occupancy stands for the occupancy of the St. 1 drift chamber.

The tracking efficiency is estimated in every $x_{2}$ region which is used in the analysis of cross section ratio. Figure 4.51 shows the occupancy dependence of the tracking efficiency. The occupancy dependence of the tracking efficiency shapes like $K_{1}(O)=1-p_{0} O^{p_{1}}$, where $O$ is the occupancy. The tracking efficiency should be larger than zero. The function $K$ is adopted as fitting function:

$$
K(O)=\frac{K_{1}(O)+\sqrt{K_{1}(O)^{2}+p_{2} O}}{p_{3} O+2} .
$$

The reasons why this function is adopted are the following:

- The shape of the function is similar to $K_{1}(O)$.

- The function is always larger than zero.

- The term of $p_{3} O$ makes the fitting better at high occupancy region.

The fitting results are shown in Fig. 4.52. The fitting parameters at each $x_{2}$ are listed in Tab. 4.10. The tracking efficiency correction factor is $1 / K$.

The errors are calculated as follows:

$$
\delta_{K}=\sqrt{\sum_{i} \sum_{j} \frac{\partial K}{\partial p_{i}} \frac{\partial K}{\partial p_{j}} \delta_{i j}}
$$




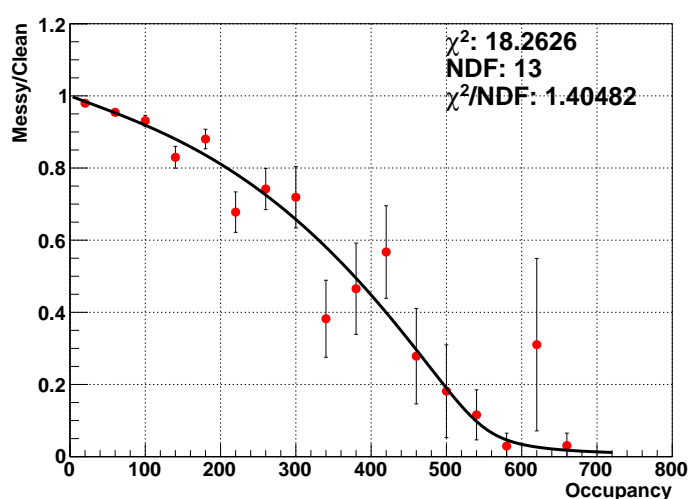

(a) $0.100<x_{2}<0.130$

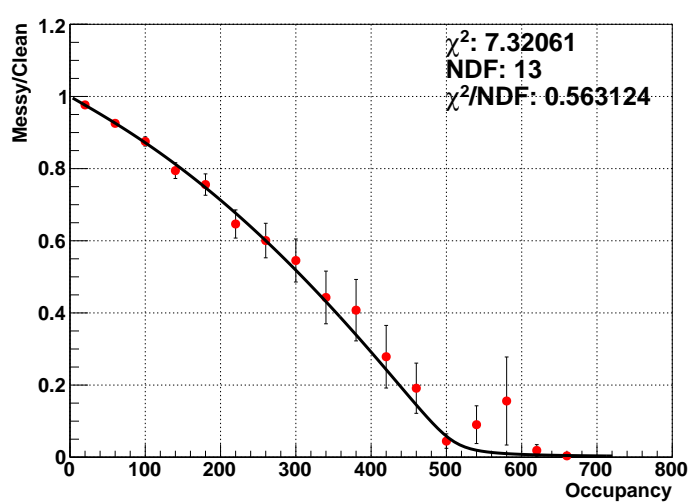

(c) $0.160<x_{2}<0.195$

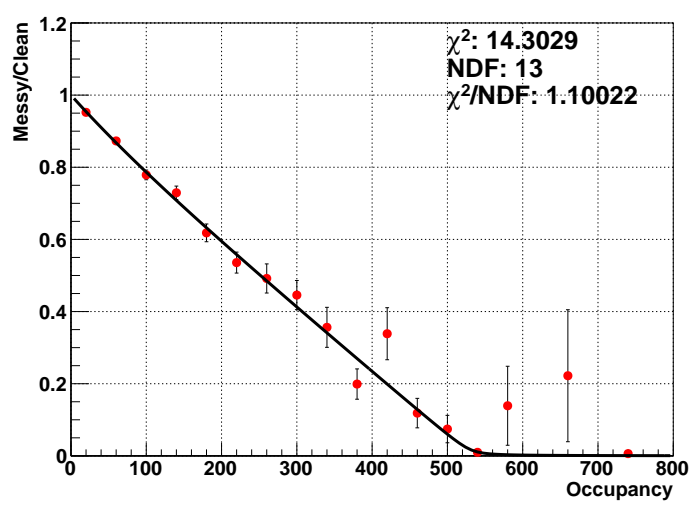

(e) $0.240<x_{2}<0.290$

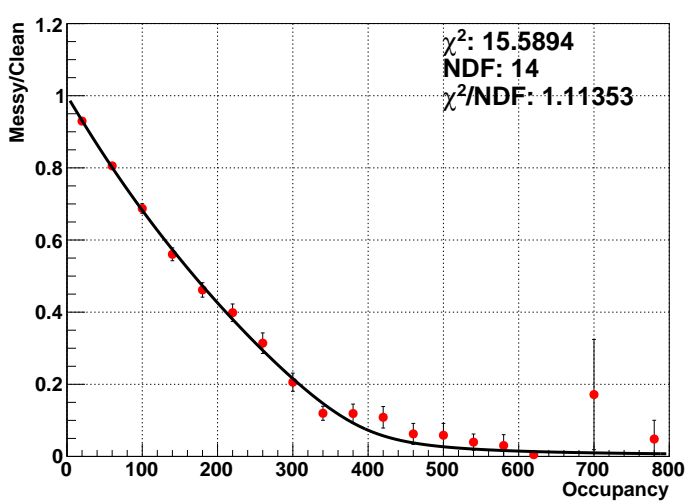

(g) $0.350<x_{2}<0.450$

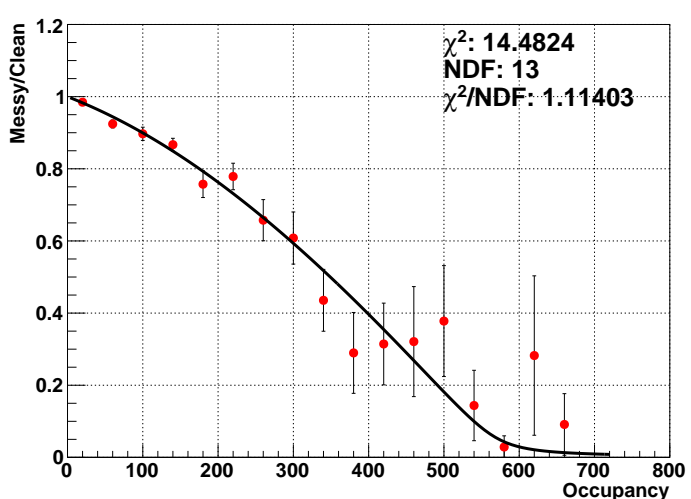

(b) $0.130<x_{2}<0.160$

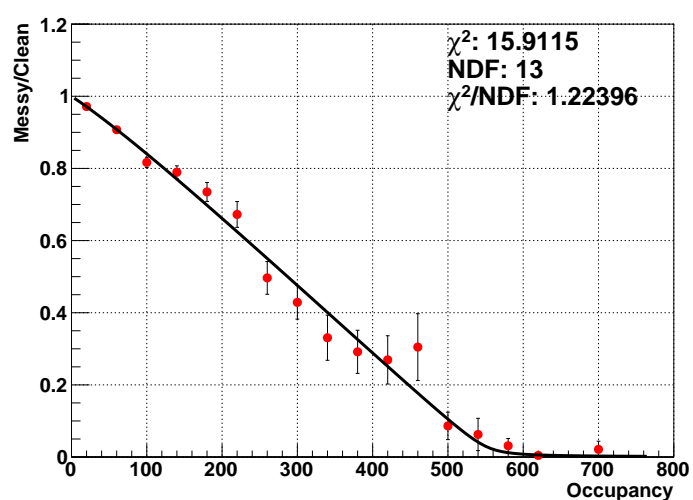

(d) $0.195<x_{2}<0.240$

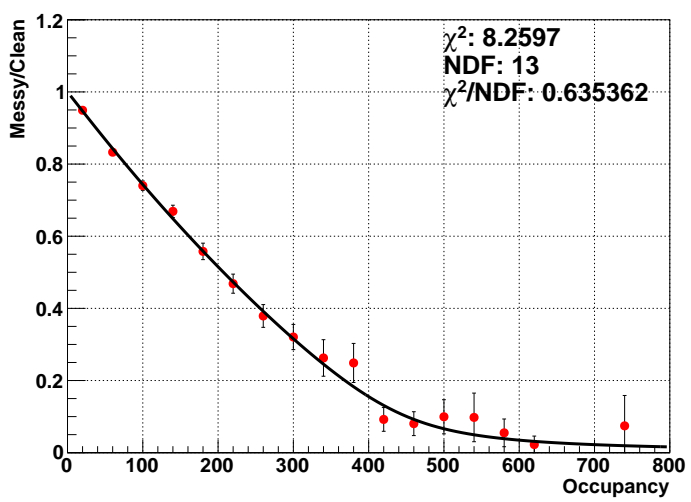

(f) $0.290<x_{2}<0.350$

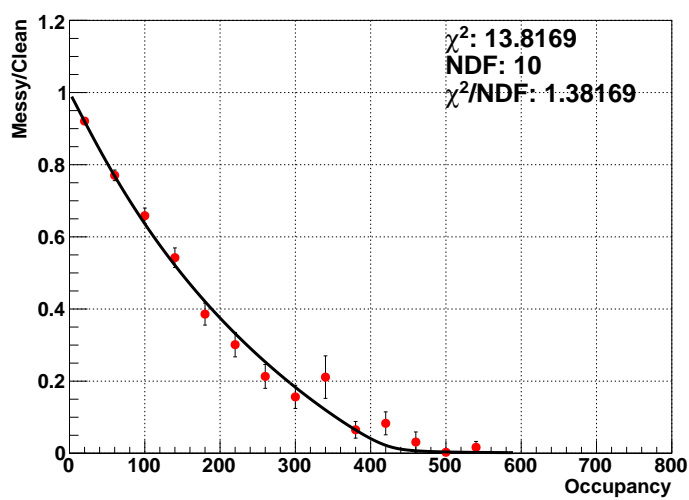

(h) $0.450<x_{2}<0.580$

Figure 4.51: Occupancy dependence of the tracking efficiency and the fitting result at each $x_{2}$. The tracking efficiency decreases as the occupancy increases. The behavior of the dependence varies in different $x_{2}$ regions. 


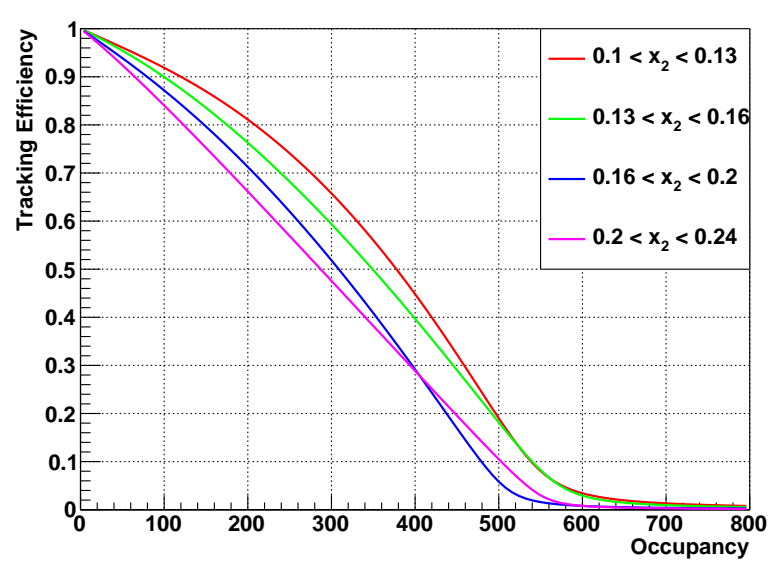

(a) $0.10<x_{2}<0.24$

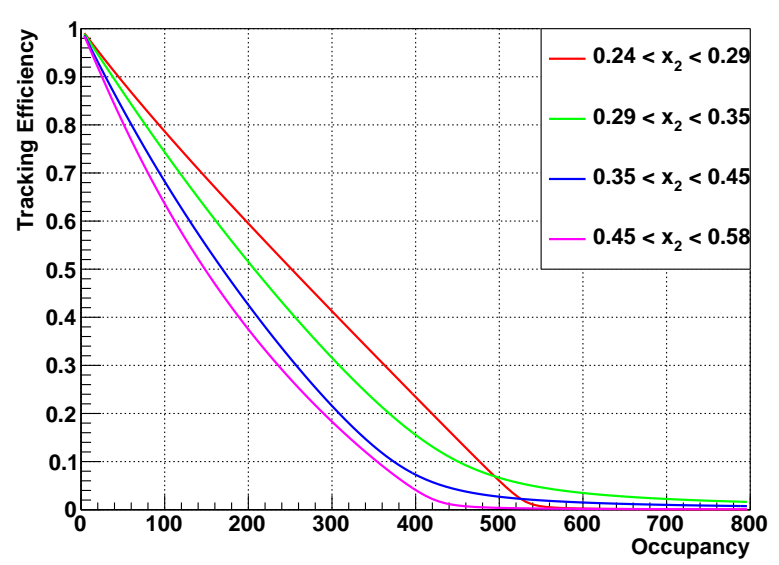

(b) $0.24<x<0.58$

Figure 4.52: Tracking efficiency as a function of occupancy at each $x_{2}$. The behavior of the dependence varies at different $x_{2}$

where $\delta_{i j}$ is the covariance of the fitting. The covariace matrix at each $x_{2}$ bin is shown in Tab. 4.11 Figure 4.53 shows the tracking efficiencies with errors at each $x_{2}$ bin. According to Fig. 4.53 (b), the value becomes less than zero at large occupancy. The events in this region are discarded.

The reason why the behavior is difference at different $x_{2}$ can be explained by the track hit position and hit distribution. Figure 4.54 shows the track hit position of $\mu^{-}$at St. 1 drift chamber in each $x_{2}$ region. The clean MC data are used. The hit position at low $x_{2}$ region is at the center of the drift chamber. The hit position at high $x_{2}$ region is at the edge of the drift chamber. Figure 4.55 shows the hit position distribution of random triggered data at St. 1 drift chamber. It includes not only the true tracks but also track un-correlated hits. The hit rate at the edge of the chamber is higher than that at the center of the chamber. Therefore, the local occupancy at the edge of the chamber is basically higher than that at the center of the chamber even if the occupancy of the chamber is the same. The tracking efficiency is affected by the hits around the true track position, and thus the effect of the occupancy on the tracking efficiency is the larger at the edge of the chamber.

Table 4.12 shows the dimuon yields after tracking efficiency correction.

Table 4.10: Fitting parameters at each $x_{2}$ bin.

\begin{tabular}{cc|cccc}
\hline$x_{2} \min$ & $x_{2} \max$ & $p_{0}$ & $p_{1}$ & $p_{2}$ & $p_{3}$ \\
\hline 0.10 & 0.13 & $2.76 \mathrm{e}-08$ & $2.76 \mathrm{e}+00$ & $1.20 \mathrm{e}-04$ & $1.64 \mathrm{e}-03$ \\
0.13 & 0.16 & $1.61 \mathrm{e}-06$ & $2.10 \mathrm{e}+00$ & $4.91 \mathrm{e}-05$ & $1.68 \mathrm{e}-03$ \\
0.16 & 0.20 & $5.90 \mathrm{e}-07$ & $2.30 \mathrm{e}+00$ & $4.37 \mathrm{e}-05$ & $2.42 \mathrm{e}-03$ \\
0.20 & 0.24 & $1.08 \mathrm{e}-05$ & $1.81 \mathrm{e}+00$ & $1.71 \mathrm{e}-05$ & $2.73 \mathrm{e}-03$ \\
0.24 & 0.29 & $1.61 \mathrm{e}-06$ & $2.13 \mathrm{e}+00$ & $1.09 \mathrm{e}-05$ & $4.71 \mathrm{e}-03$ \\
0.29 & 0.35 & $1.38 \mathrm{e}-05$ & $1.84 \mathrm{e}+00$ & $5.18 \mathrm{e}-04$ & $5.52 \mathrm{e}-03$ \\
0.35 & 0.45 & $2.15 \mathrm{e}-05$ & $1.80 \mathrm{e}+00$ & $3.71 \mathrm{e}-04$ & $7.10 \mathrm{e}-03$ \\
0.45 & 0.58 & $7.71 \mathrm{e}-04$ & $1.18 \mathrm{e}+00$ & $1.62 \mathrm{e}-05$ & $5.79 \mathrm{e}-03$ \\
\hline
\end{tabular}


Table 4.11: Covariance matrix at each $x_{2}$ bin.

\begin{tabular}{|c|c|c|c|c|c|c|c|c|}
\hline & \multicolumn{4}{|c|}{$x_{2}: 0.10-0.13$} & \multicolumn{4}{|c|}{$x_{2}: 0.13-0.16$} \\
\hline & $p_{0}$ & $p_{1}$ & $p_{2}$ & $p_{3}$ & $p_{0}$ & $p_{1}$ & $p_{2}$ & $p_{3}$ \\
\hline$p_{0}$ & $2.48 e-15$ & $-1.49 e-08$ & $-3.19 e-12$ & $-6.70 e-12$ & $4.72 \mathrm{e}-12$ & $-4.91 e-07$ & $1.17 \mathrm{e}-10$ & $-3.90 e-10$ \\
\hline$p_{1}$ & $-1.49 \mathrm{e}-08$ & $9.03 e-02$ & $2.42 \mathrm{e}-05$ & $4.05 \mathrm{e}-05$ & $-4.91 e-07$ & $5.19 \mathrm{e}-02$ & $-1.51 \mathrm{e}-05$ & $3.92 \mathrm{e}-05$ \\
\hline$p_{2}$ & $-3.19 \mathrm{e}-12$ & $2.42 \mathrm{e}-05$ & $3.74 \mathrm{e}-08$ & $1.63 \mathrm{e}-08$ & $1.17 \mathrm{e}-10$ & $-1.51 e-05$ & $1.76 \mathrm{e}-08$ & $-6.67 e-09$ \\
\hline \multirow[t]{3}{*}{$p_{3}$} & $-6.70 \mathrm{e}-12$ & $4.05 e-05$ & $1.63 \mathrm{e}-08$ & $5.01 \mathrm{e}-08$ & $-3.90 \mathrm{e}-10$ & $3.92 \mathrm{e}-05$ & $-6.67 e-09$ & $6.06 \mathrm{e}-08$ \\
\hline & \multicolumn{4}{|c|}{$x_{2}: 0.16-0.20$} & \multicolumn{4}{|c|}{$x_{2}: 0.20-0.24$} \\
\hline & $p_{0}$ & $p_{1}$ & $p_{2}$ & $p_{3}$ & $p_{0}$ & $p_{1}$ & $p_{2}$ & $p_{3}$ \\
\hline$p_{0}$ & $5.16 \mathrm{e}-13$ & $-1.43 e-07$ & $-7.37 \mathrm{e}-12$ & $-1.11 \mathrm{e}-10$ & $1.44 \mathrm{e}-10$ & $-2.17 e-06$ & $-7.18 \mathrm{e}-11$ & $-3.29 \mathrm{e}-09$ \\
\hline$p_{1}$ & $-1.43 \mathrm{e}-07$ & $3.96 \mathrm{e}-02$ & $2.27 \mathrm{e}-06$ & $3.05 \mathrm{e}-05$ & $-2.17 e-06$ & $3.28 \mathrm{e}-02$ & $1.17 \mathrm{e}-06$ & $4.86 \mathrm{e}-05$ \\
\hline$p_{2}$ & $-7.37 \mathrm{e}-12$ & $2.27 \mathrm{e}-06$ & $1.40 \mathrm{e}-09$ & $1.67 \mathrm{e}-09$ & $-7.18 \mathrm{e}-11$ & $1.17 \mathrm{e}-06$ & $1.92 \mathrm{e}-10$ & $1.13 \mathrm{e}-09$ \\
\hline \multirow{3}{*}{$p_{3}$} & $-1.11 \mathrm{e}-10$ & $3.05 e-05$ & $1.67 \mathrm{e}-09$ & $4.77 \mathrm{e}-08$ & $-3.29 \mathrm{e}-09$ & $4.86 \mathrm{e}-05$ & $1.13 \mathrm{e}-09$ & $1.06 \mathrm{e}-07$ \\
\hline & \multicolumn{4}{|c|}{$x_{2}: 0.24-0.29$} & \multicolumn{4}{|c|}{$x_{2}: 0.29-0.35$} \\
\hline & $p_{0}$ & $p_{1}$ & $p_{2}$ & $p_{3}$ & $p_{0}$ & $p_{1}$ & $p_{2}$ & $p_{3}$ \\
\hline$p_{0}$ & $7.77 e-13$ & $-7.88 \mathrm{e}-08$ & $-2.54 \mathrm{e}-12$ & $-8.99 \mathrm{e}-11$ & $9.60 \mathrm{e}-10$ & $-1.25 \mathrm{e}-05$ & $-1.46 e-08$ & $-2.77 \mathrm{e}-08$ \\
\hline$p_{1}$ & $-7.88 \mathrm{e}-08$ & $8.13 \mathrm{e}-03$ & $4.50 \mathrm{e}-07$ & $8.19 \mathrm{e}-06$ & $-1.25 e-05$ & $1.62 \mathrm{e}-01$ & $1.98 \mathrm{e}-04$ & $3.60 \mathrm{e}-04$ \\
\hline$p_{2}$ & $-2.54 \mathrm{e}-12$ & $4.50 \mathrm{e}-07$ & $3.45 \mathrm{e}-10$ & $-7.69 \mathrm{e}-10$ & $-1.46 e-08$ & $1.98 \mathrm{e}-04$ & $3.57 \mathrm{e}-07$ & $4.55 \mathrm{e}-07$ \\
\hline \multirow[t]{3}{*}{$p_{3}$} & $-8.99 \mathrm{e}-11$ & $8.19 \mathrm{e}-06$ & $-7.69 e-10$ & $5.12 \mathrm{e}-08$ & $-2.77 e-08$ & $3.60 \mathrm{e}-04$ & $4.55 \mathrm{e}-07$ & $8.62 \mathrm{e}-07$ \\
\hline & \multicolumn{4}{|c|}{$x_{2}: 0.35-0.45$} & \multicolumn{4}{|c|}{$x_{2}: 0.45-0.58$} \\
\hline & $p_{0}$ & $p_{1}$ & $p_{2}$ & $p_{3}$ & $p_{0}$ & $p_{1}$ & $p_{2}$ & $p_{3}$ \\
\hline$p_{0}$ & $1.83 e-09$ & $-1.51 \mathrm{e}-05$ & $-1.09 \mathrm{e}-08$ & $-3.96 e-08$ & $5.22 \mathrm{e}-07$ & $-1.12 \mathrm{e}-04$ & $-3.23 e-09$ & $-9.33 e-07$ \\
\hline$p_{1}$ & $-1.51 \mathrm{e}-05$ & $1.25 \mathrm{e}-01$ & $9.20 \mathrm{e}-05$ & $3.26 \mathrm{e}-04$ & $-1.12 \mathrm{e}-04$ & $2.41 \mathrm{e}-02$ & 7.80e-07 & $1.96 \mathrm{e}-04$ \\
\hline$p_{2}$ & $-1.09 \mathrm{e}-08$ & $9.20 \mathrm{e}-05$ & $8.80 \mathrm{e}-08$ & $2.35 \mathrm{e}-07$ & $-3.23 e-09$ & $7.80 \mathrm{e}-07$ & $1.51 \mathrm{e}-10$ & $3.02 \mathrm{e}-09$ \\
\hline$p_{3}$ & $-3.96 e-08$ & $3.26 \mathrm{e}-04$ & $2.35 \mathrm{e}-07$ & $9.37 \mathrm{e}-07$ & $-9.33 e-07$ & $1.96 \mathrm{e}-04$ & $3.02 \mathrm{e}-09$ & $1.97 \mathrm{e}-06$ \\
\hline
\end{tabular}

Table 4.12: Dimuon corrected yields.

\begin{tabular}{c|c|rrr}
\hline$x_{2} \min$ & $x_{2} \max$ & $\mathrm{LH}_{2}$ & $\mathrm{LD}_{2}$ & Empty \\
\hline 0.10 & 0.13 & 1700.56 & 1995.89 & 76.90 \\
0.13 & 0.16 & 4804.30 & 5494.04 & 201.28 \\
0.16 & 0.20 & 6035.03 & 7085.38 & 250.08 \\
0.20 & 0.24 & 5064.06 & 5642.20 & 198.93 \\
0.24 & 0.29 & 2969.89 & 3346.48 & 125.35 \\
0.29 & 0.35 & 1656.86 & 1881.93 & 72.95 \\
0.35 & 0.45 & 770.91 & 927.54 & 48.16 \\
0.45 & 0.58 & 156.01 & 155.94 & 5.13 \\
\hline Sum & & 23157.62 & 26529.40 & 978.78 \\
\hline
\end{tabular}




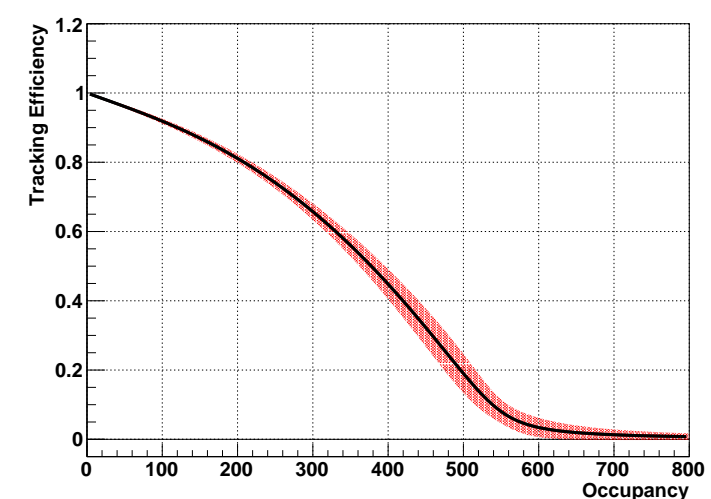

(a) $0.100<x_{2}<0.130$

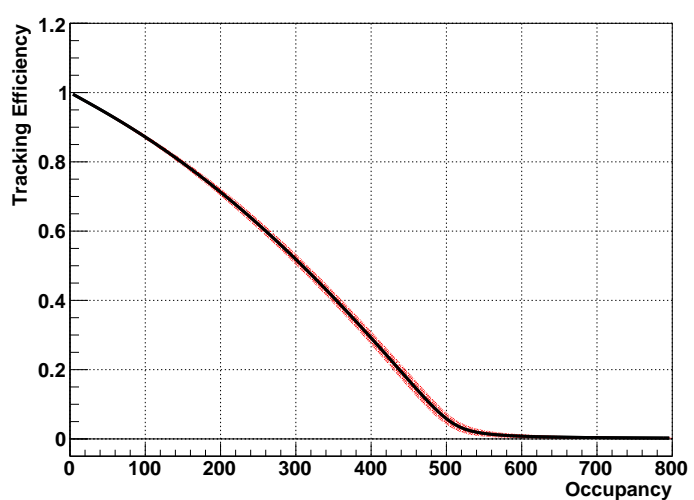

(c) $0.160<x_{2}<0.195$

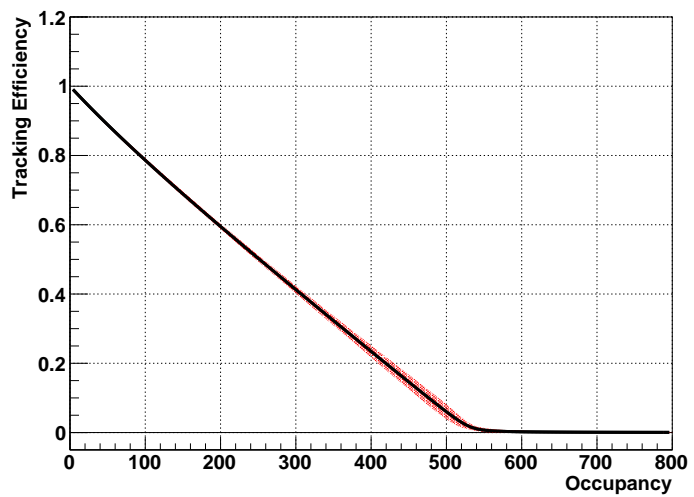

(e) $0.240<x_{2}<0.290$

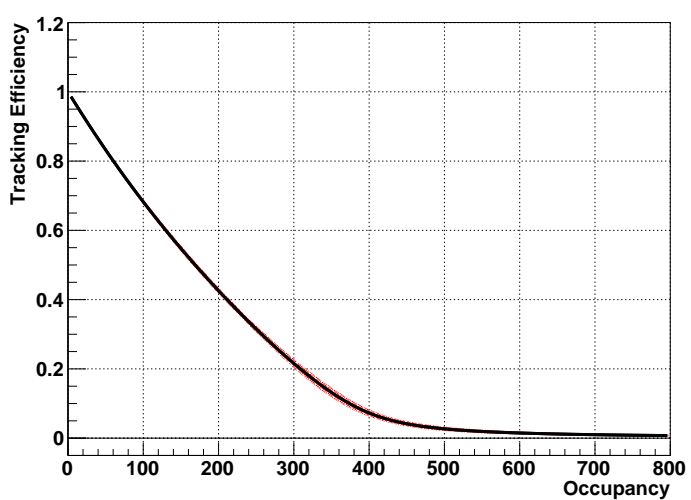

(g) $0.350<x_{2}<0.450$

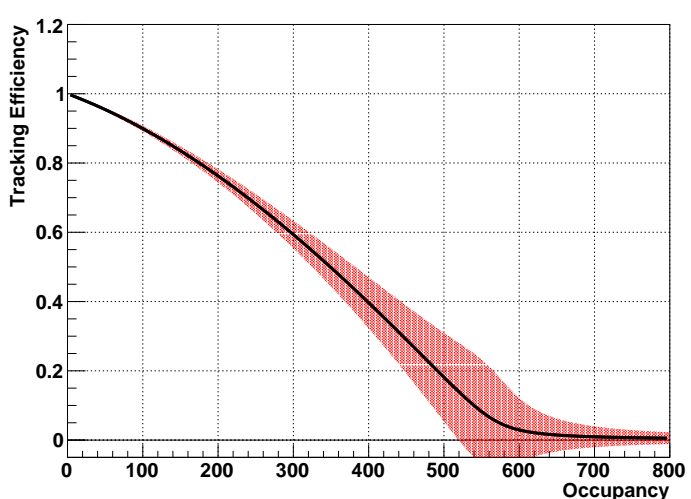

(b) $0.130<x_{2}<0.160$

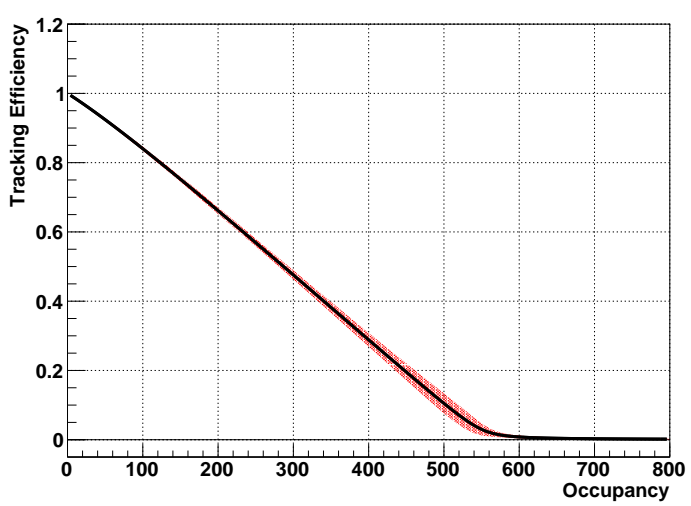

(d) $0.195<x_{2}<0.240$

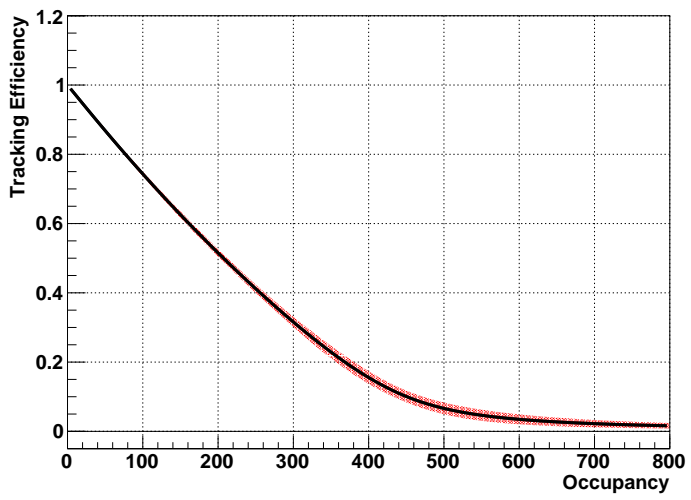

(f) $0.290<x_{2}<0.350$



(h) $0.450<x_{2}<0.580$

Figure 4.53: Tracking efficiencies with errors at each $x_{2}$ bin. 

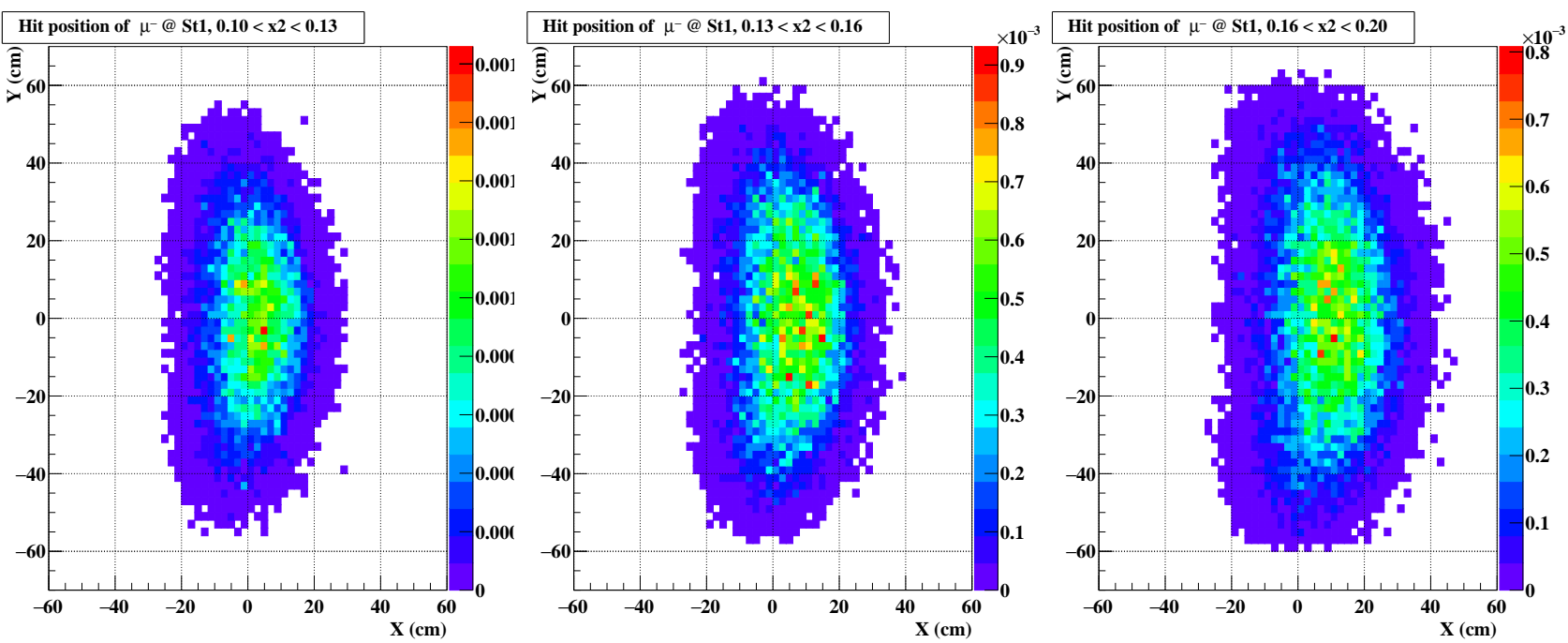

(a) $0.100<x_{2}<0.130$

(b) $0.130<x_{2}<0.160$

(c) $0.160<x_{2}<0.195$
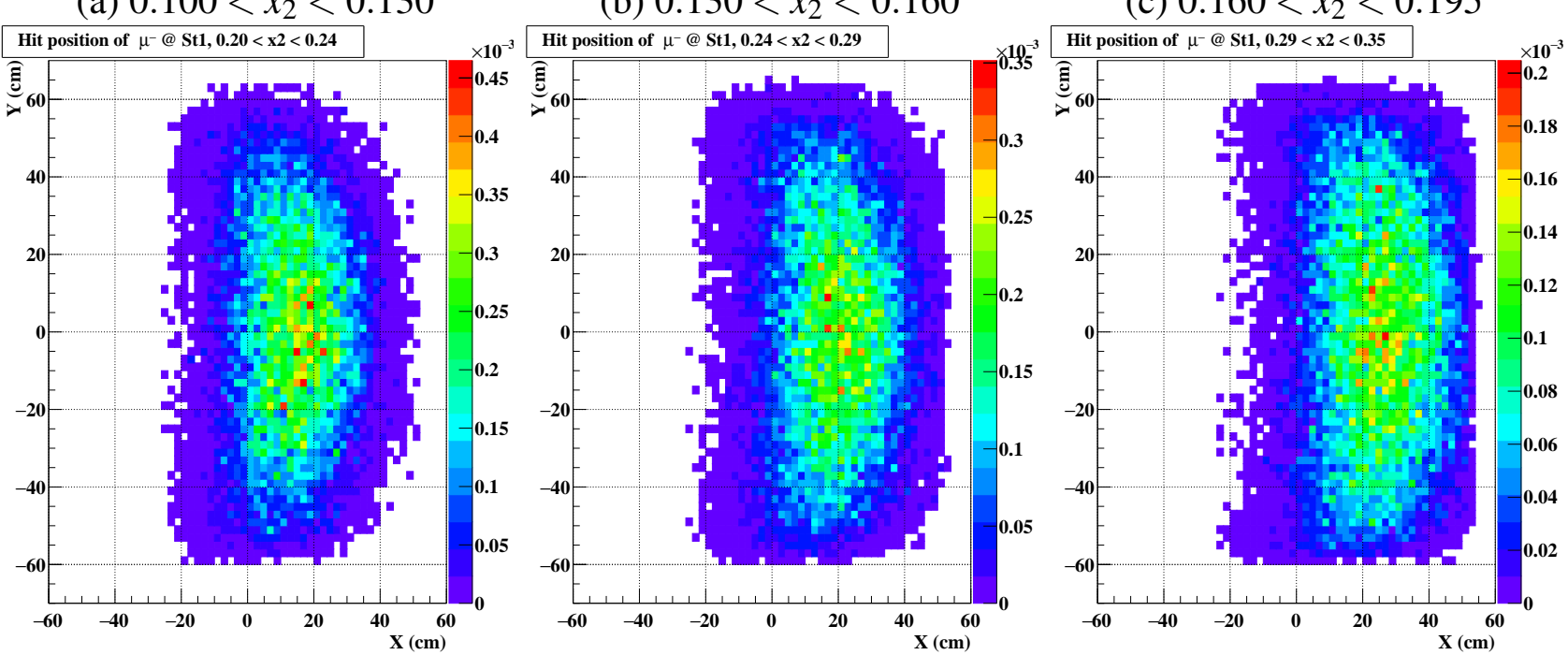

(d) $0.195<x_{2}<0.240$

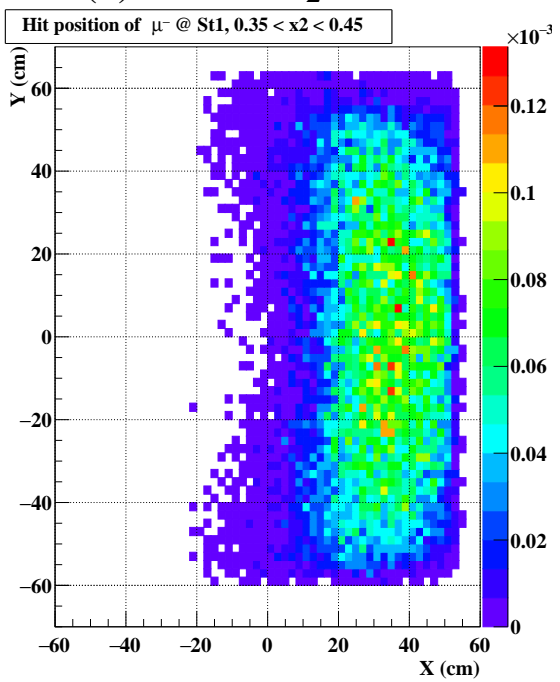

(e) $0.240<x_{2}<0.290$

(f) $0.290<x_{2}<0.350$

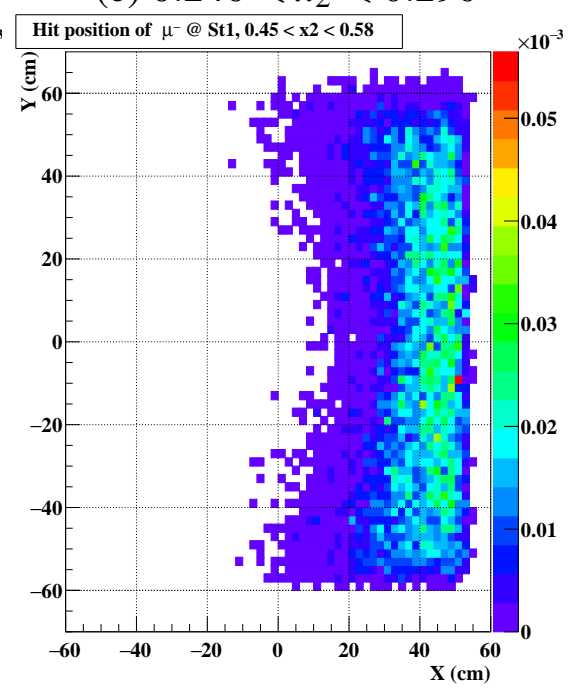

(g) $0.350<x_{2}<0.450$

(h) $0.450<x_{2}<0.580$

Figure 4.54: Track hit position of $\mu^{-}$at St. 1 drift chamber. The clean MC data are used. The hit distribution varies in each $x_{2}$ region. 


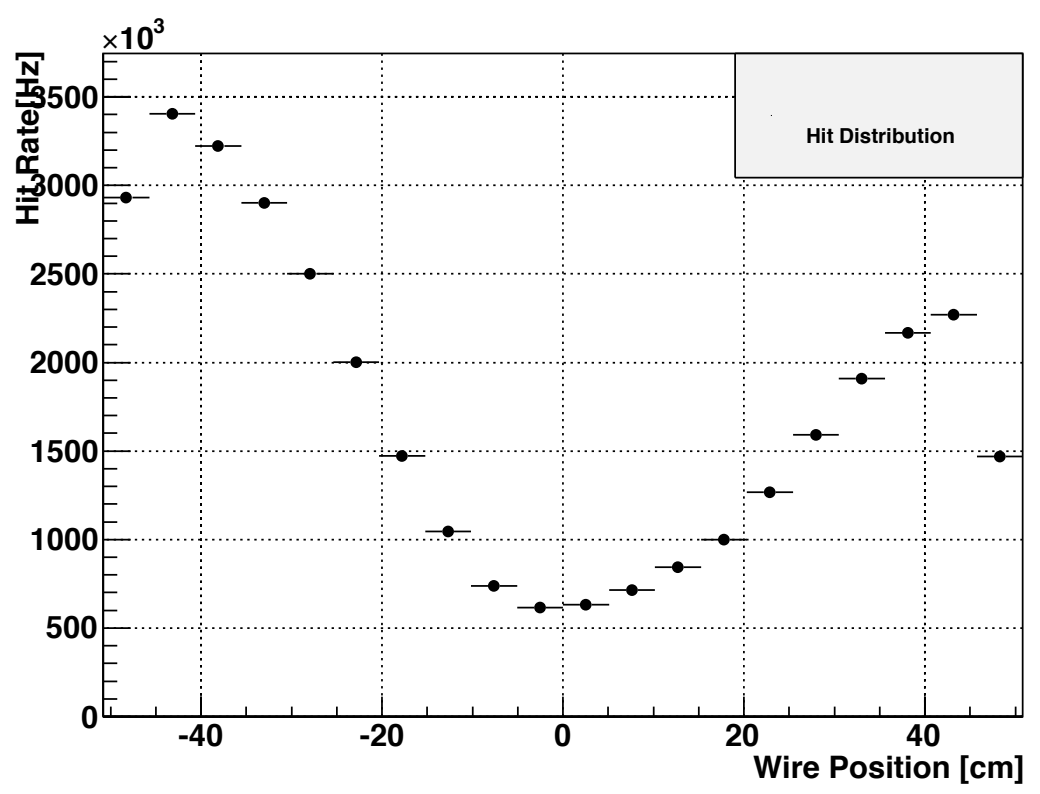

Figure 4.55: Hit distribution at St. 1 drift chamber. The random triggered real data are used. 


\subsubsection{Empty Target Subtraction}

The dimuon yields of $\mathrm{LD}_{2}$ and $\mathrm{LH}_{2}$ targets include events coming from the dump, the target flask edge, and upstream of the targets. The empty target data are taken in order to subtract these dimuons. Figure 4.56 shows the relation between the occupancy and $x_{2}$ of each target data. Figure 4.57 shows

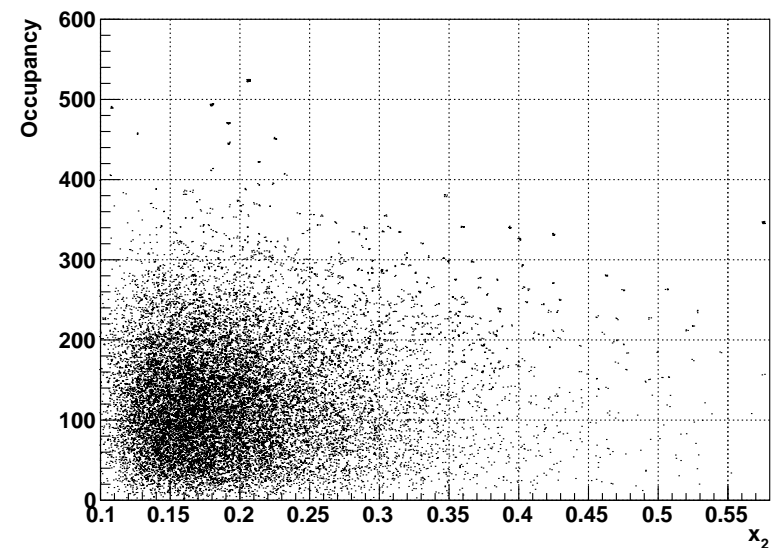

(a) $\mathrm{LH}_{2}$

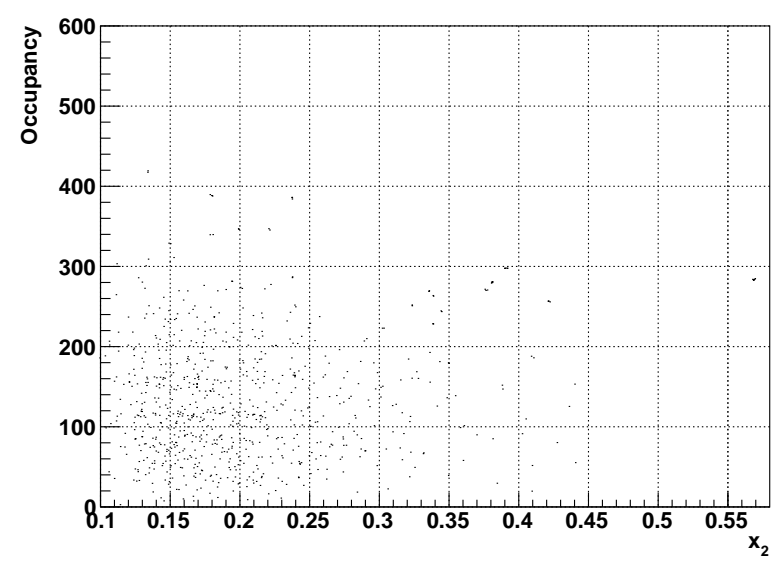

(c) Empty Target

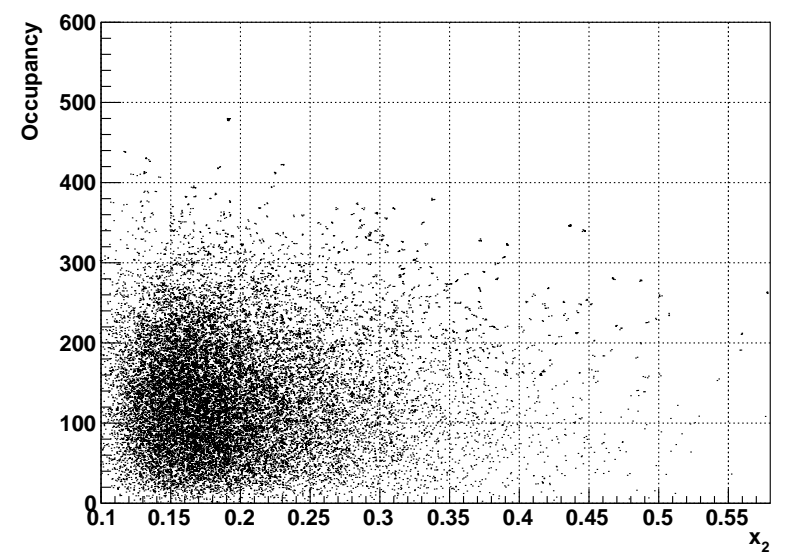

(b) $\mathrm{LD}_{2}$

Figure 4.56: Relation between the occupancy and $x_{2}$.

the dimuon yields of $\mathrm{LH}_{2}, \mathrm{LD}_{2}$ and empty target. Each distribution is normalized with the number of beam protons of each target data. The events at high occupancy events (occupancy $>350$ ) are few and therefore the characteristics cannot be well investigated. Thus, the additional selection is applied: occupancy $<350$. The number of events with occupancy $>350$ is about $1 \%$ of protons on target for each $\mathrm{LH}_{2}, \mathrm{LD}_{2}$ and empty target data. Therefore, this cut does not affect the result significantly.

The method to subtract the empty target events is as follows:

- Apply the tracking efficiency correction based on the occupancy in the same way as $\mathrm{LH}_{2}$ and $\mathrm{LD}_{2}$ targets.

- Normalize the empty target events with protons on target.

- Subtract the empty target events from $\mathrm{LH}_{2}$ or $\mathrm{LD}_{2}$ target events $x_{2}$-bin-by-bin. 

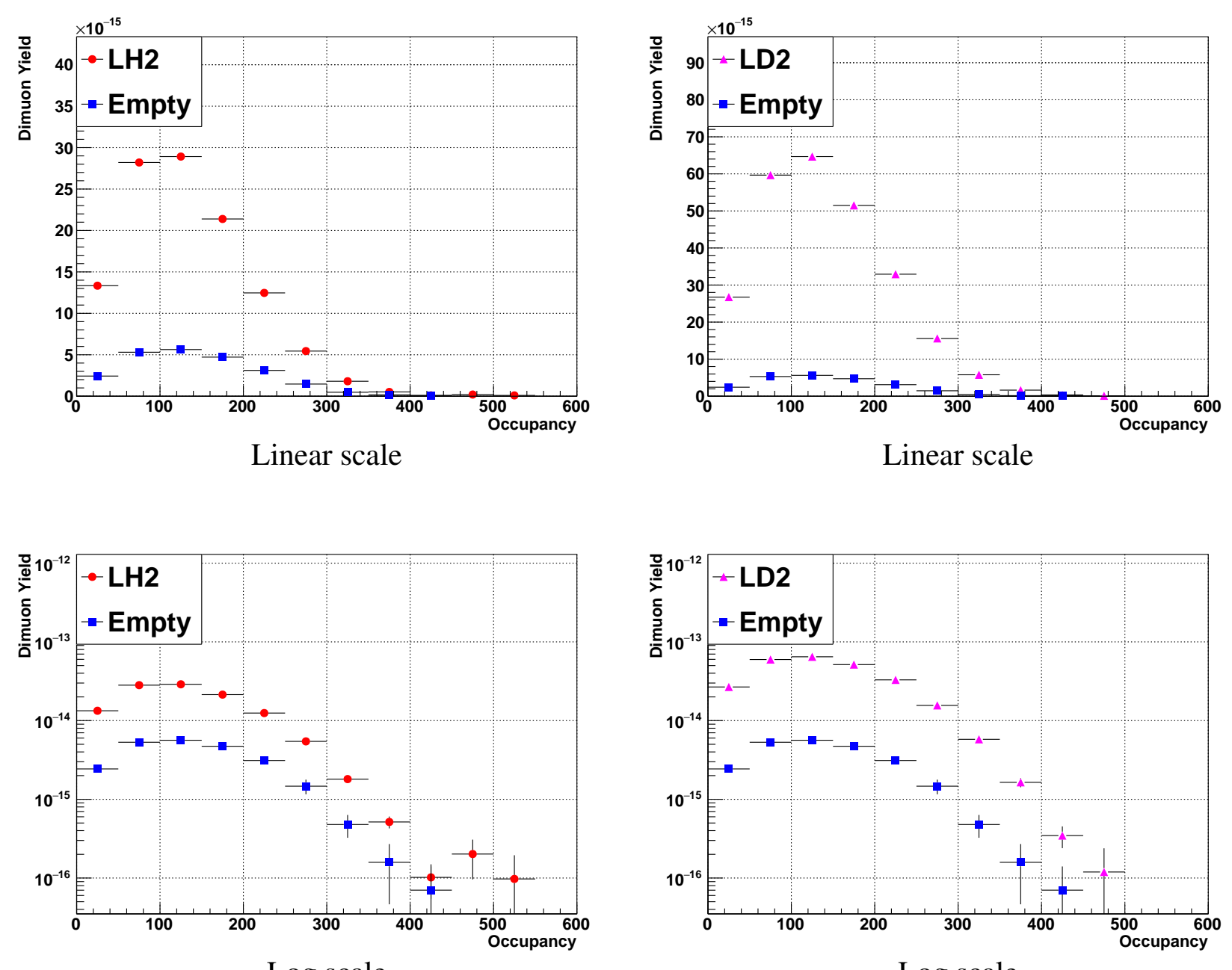

Log scale

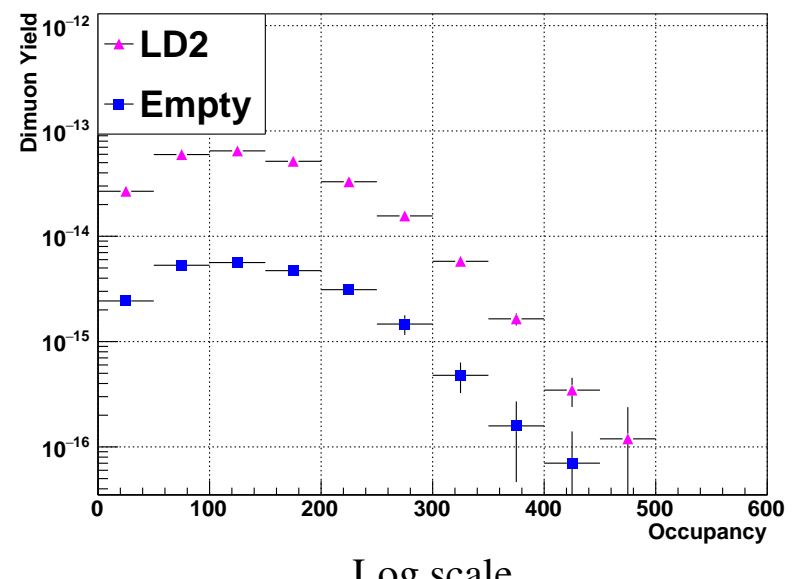

(a) $\mathrm{LH}_{2}$

Log scale

(b) $\mathrm{LD}_{2}$

Figure 4.57: Dimuon yields of $\mathrm{LH}_{2}, \mathrm{LD}_{2}$ and empty target. Each distribution is normalized with the number of beam protons of each target data. Top figures are plotted in linear scale while bottom figures are plotted in log scale. Empty target data are common in all these plots. 
Here, the empty target events are subtracted occupancy-bin-by-bin.

Figure 4.58 shows the dimuon yield ratio of $\mathrm{LD}_{2}$ to $\mathrm{LH}_{2}$ as a function of the occupancy after the tracking efficiency correction and the empty target subtraction. The dimuon yield ratio is stable in the

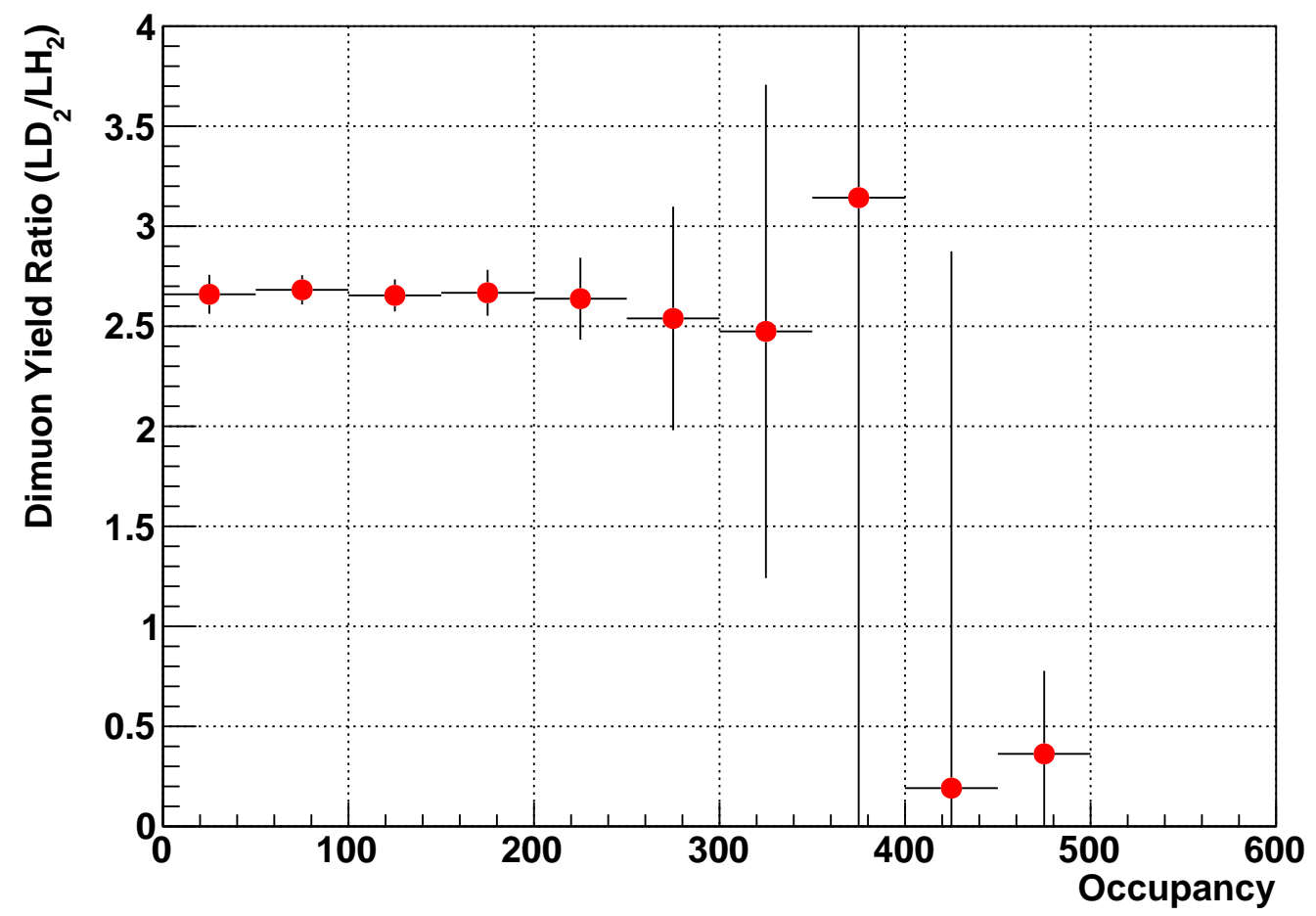

Figure 4.58: Dimuon yield ratio of $\mathrm{LD}_{2}$ to $\mathrm{LH}_{2}$ as a function of the occupancy after the tracking efficiency correction and the empty target subtraction. Events at the bins with no entry don't exist.

range of occupancy $<350$. Figure 4.59 shows the dimuon yield ratio of $\mathrm{LD}_{2}$ to $\mathrm{LH}_{2}$ as a function of the occupancy range. The occupancy range dependence of the dimuon yield ratio is stable for overall occupancy range. The statistics at occupancy $>350$ are few, so it does not affect the result.

The stability of the dimuon yield ratio at each $x_{2}$ bin is also investigated since the extractions of the cross section ratio and $\bar{d} / \bar{u}$ are performed in each $x_{2}$ bin. Figure 4.60 shows the dimuon yield ratio as a function of occupancy in each $x_{2}$ bin. The largest three $x_{2}$ bins are combined because the statistics is low. The dimuon yield ratio is stable within the error in overall occupancy range. 


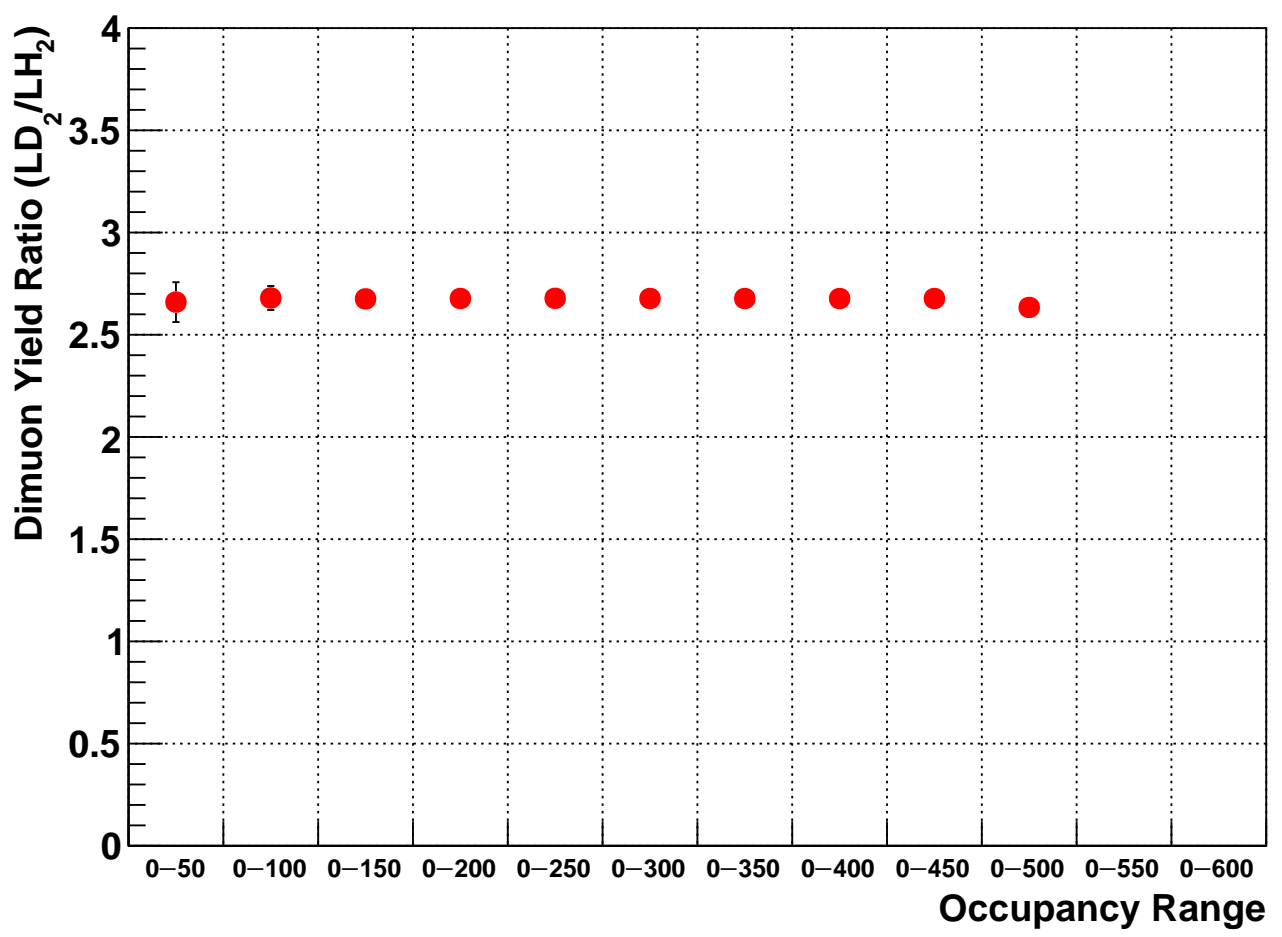

Figure 4.59: Dimuon yield ratio of $\mathrm{LD}_{2}$ to $\mathrm{LH}_{2}$ as a function of the occupancy range. 


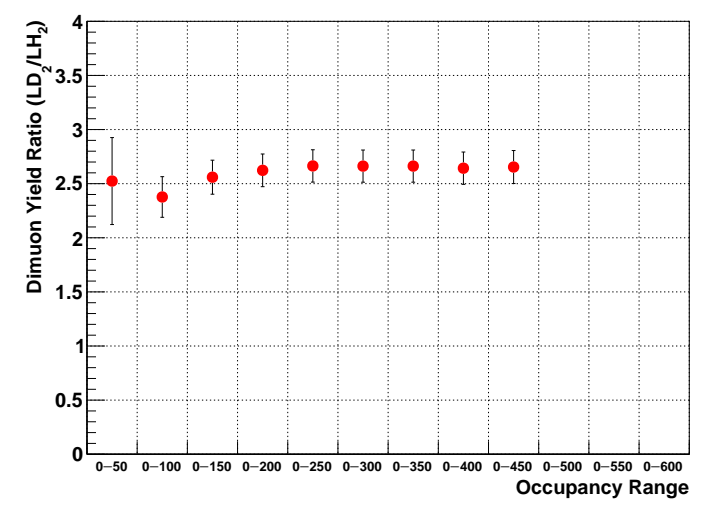

(a) $0.100<x_{2}<0.130$

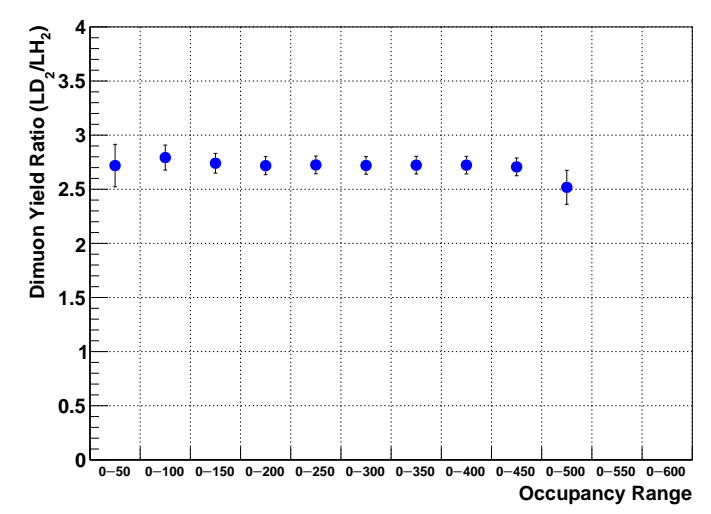

(c) $0.160<x_{2}<0.195$

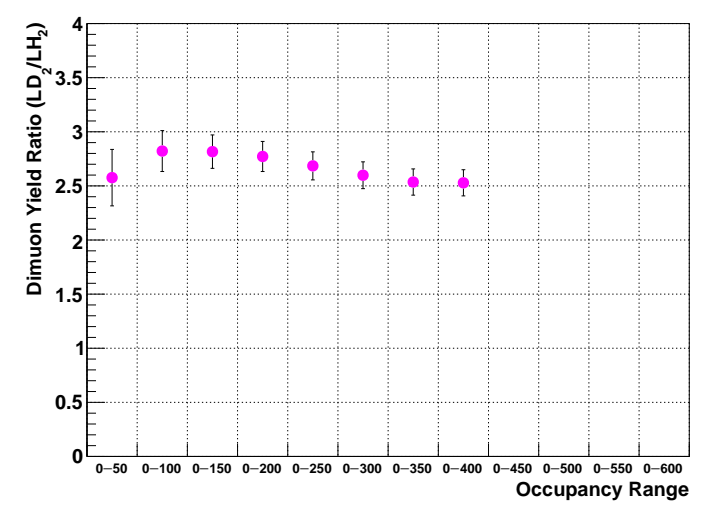

(e) $0.240<x_{2}<0.290$

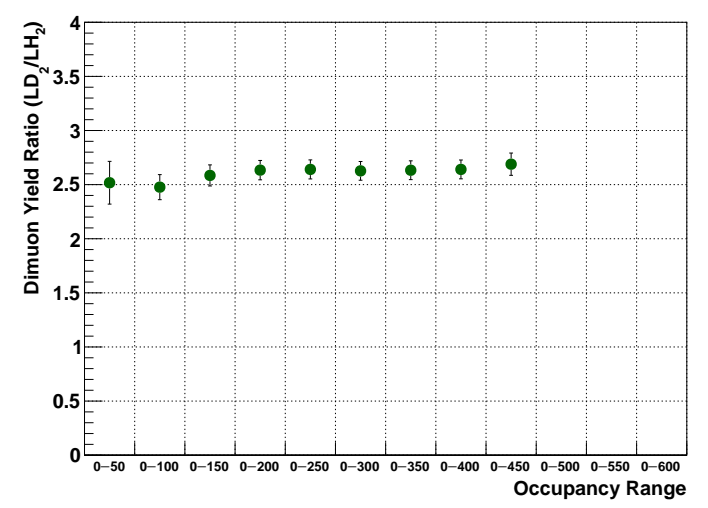

(b) $0.130<x_{2}<0.160$

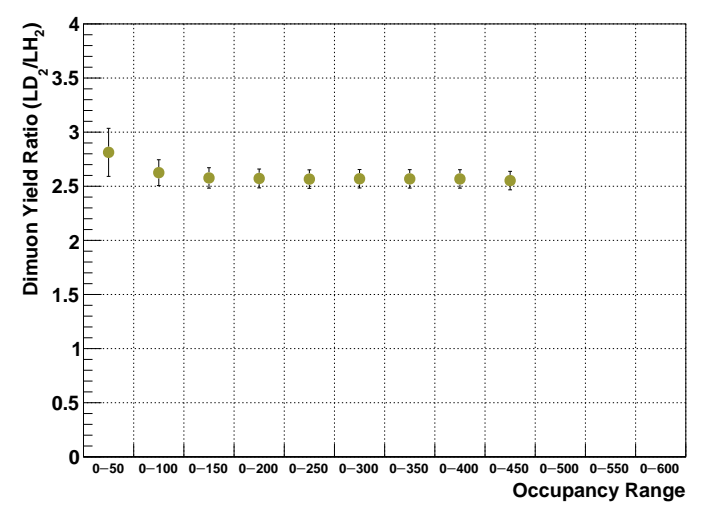

(d) $0.195<x_{2}<0.240$

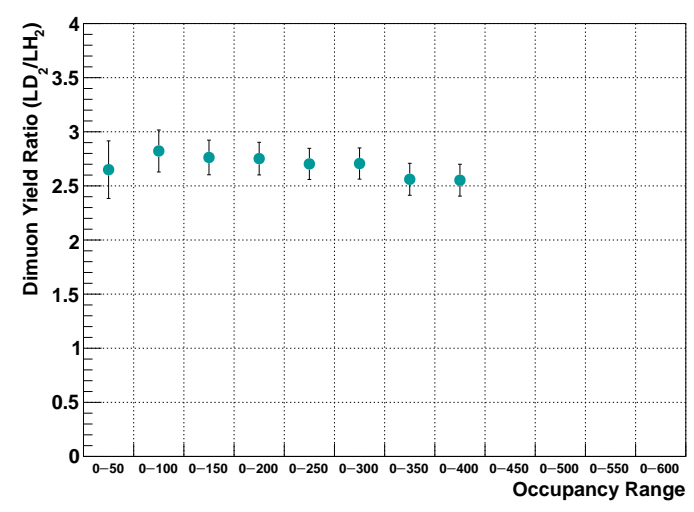

(f) $0.290<x_{2}<0.580$

Figure 4.60: Dimuon yield ratio as a function of occupancy in each $x_{2}$ bin. The largest three $x_{2}$ bins are combined due to the statistics. 


\subsubsection{Occupancy Dependence of Combinatorial Background}

The number of combinatorial backgrounds depends on the occupancy since the number of tracks becomes more when the beam intensity is higher. In order to investigate the occupancy dependence of the combinatorial background, the mass fitting is performed in each occupancy bin. Figure 4.61 and Fig. 4.62 show the mass distributions of $\mathrm{LH}_{2}$ and $\mathrm{LD}_{2}$ targets events in each occupancy bin, respectively. The ratio of the combinatorial background to the Drell-Yan dimuons is shown in Fig. 4.63. The ratios of $\mathrm{LH}_{2}$ and $\mathrm{LD}_{2}$ are consistent. The fraction of the combinatorial background is cancelled out by taking the ratio of $\mathrm{LD}_{2}$ and $\mathrm{LH}_{2}$ dimuon yields. Therefore, no correction is needed. 


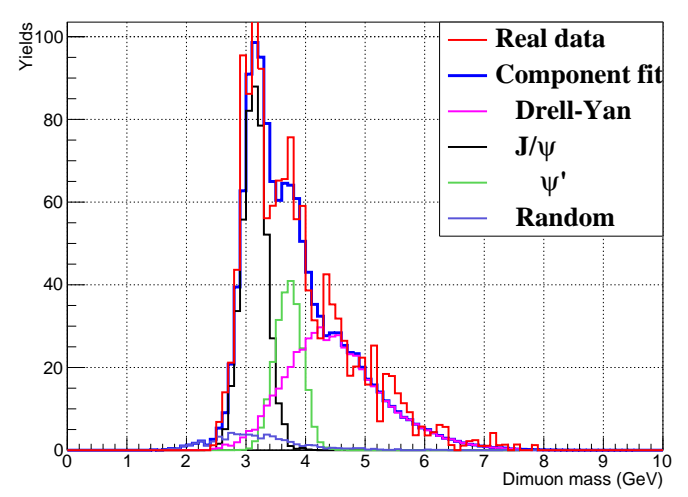

(a) Occupancy $=0-50$

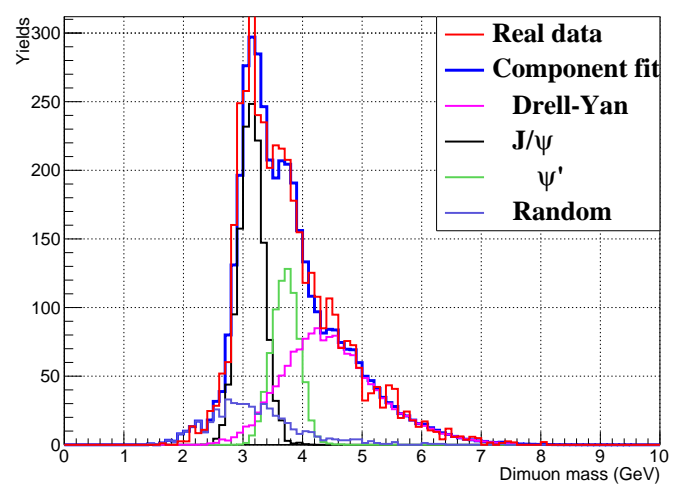

(c) Occupancy $=100-150$

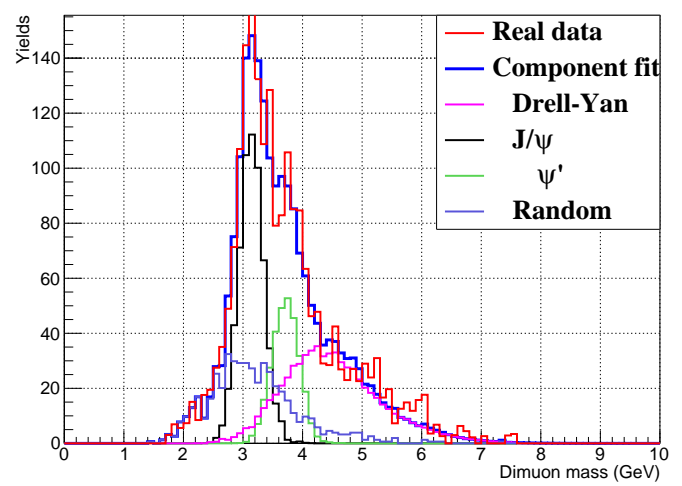

(e) Occupancy $=200-250$

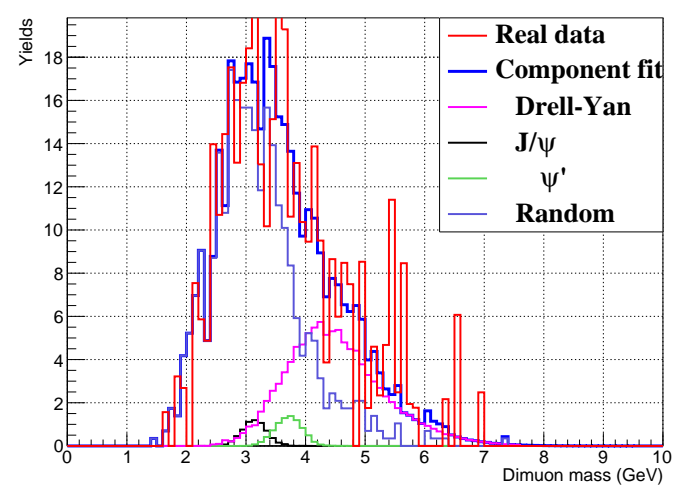

(g) Occupancy $=300-350$

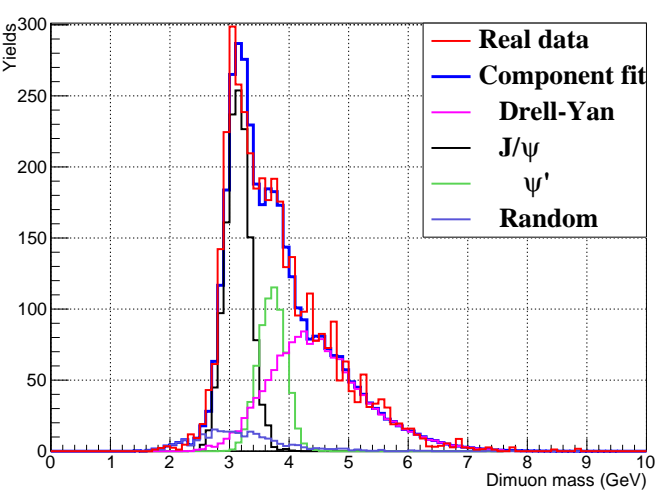

(b) Occupancy $=50-100$

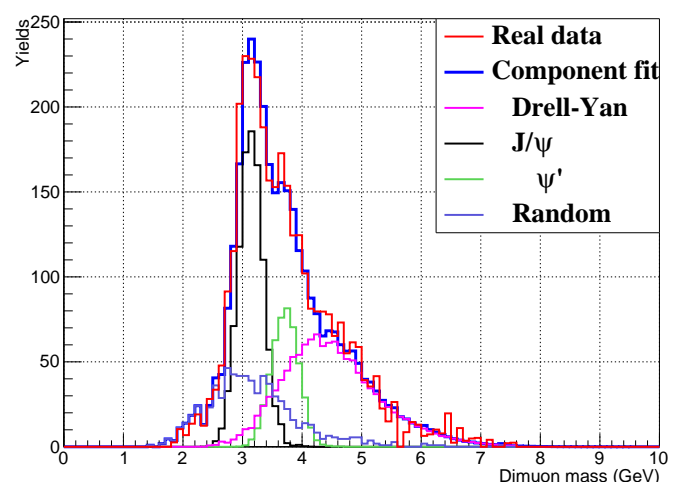

(d) Occupancy=150-200

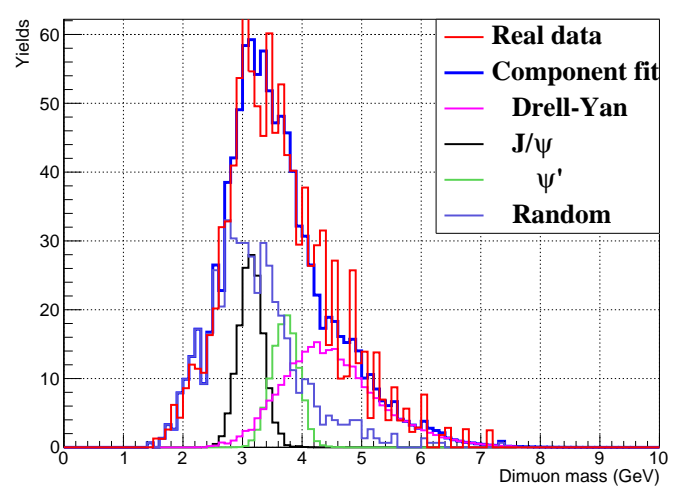

(f) Occupancy $=250-300$

Figure 4.61: Mass distribution of LH24arget events in each occupancy bin. 


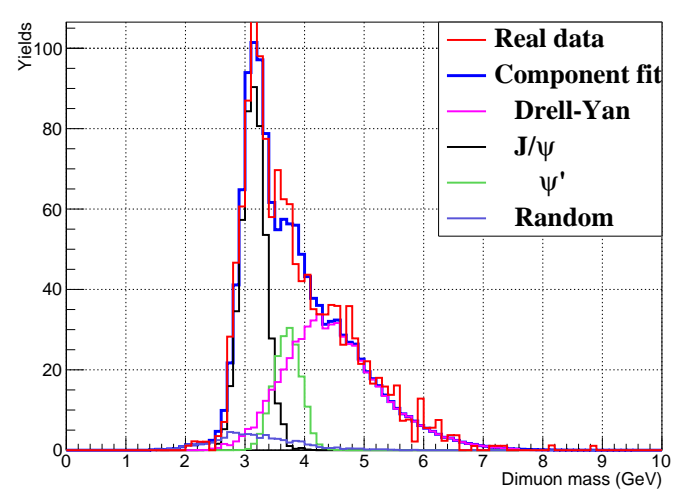

(a) Occupancy $=0-50$

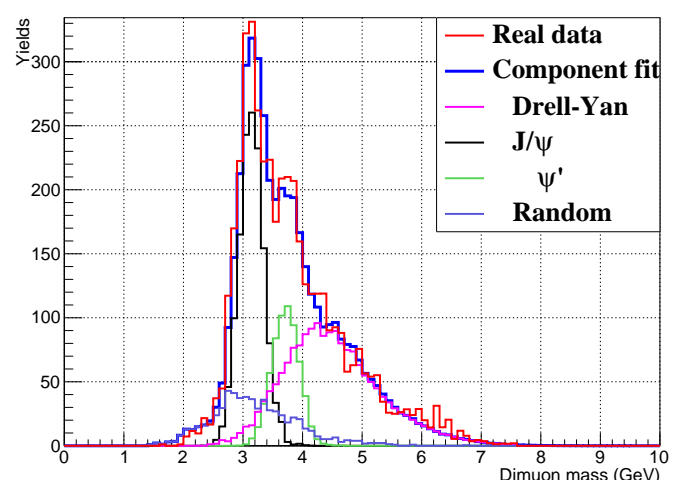

(c) Occupancy $=100-150$

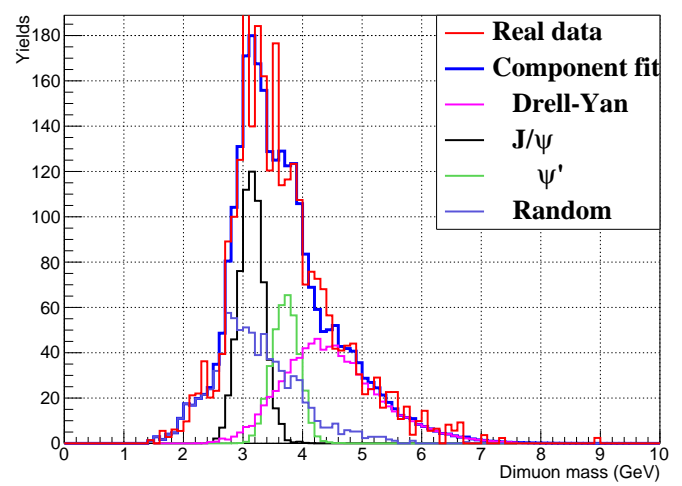

(e) Occupancy $=200-250$

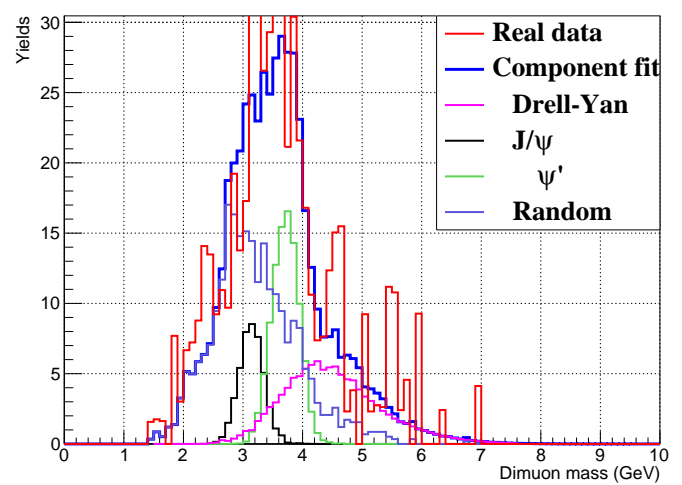

(g) Occupancy $=300-350$

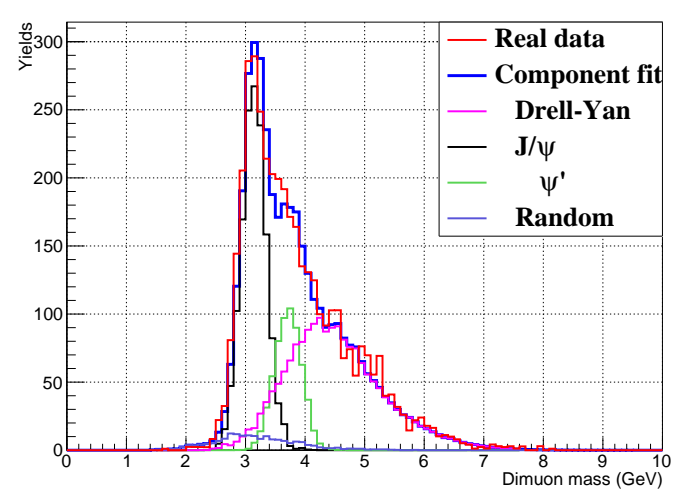

(b) Occupancy $=50-100$

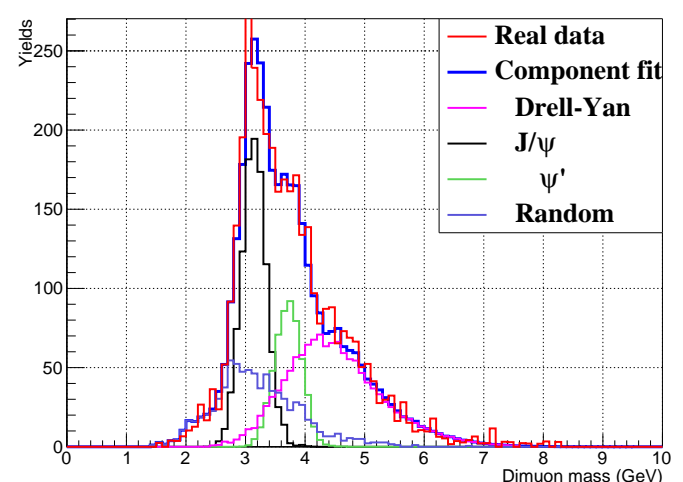

(d) Occupancy=150-200

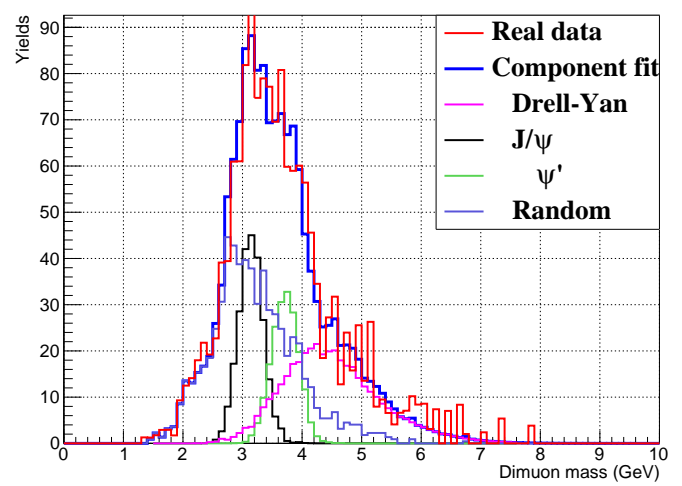

(f) Occupancy $=250-300$

Figure 4.62: Mass distribution of LD25arget events in each occupancy bin. 


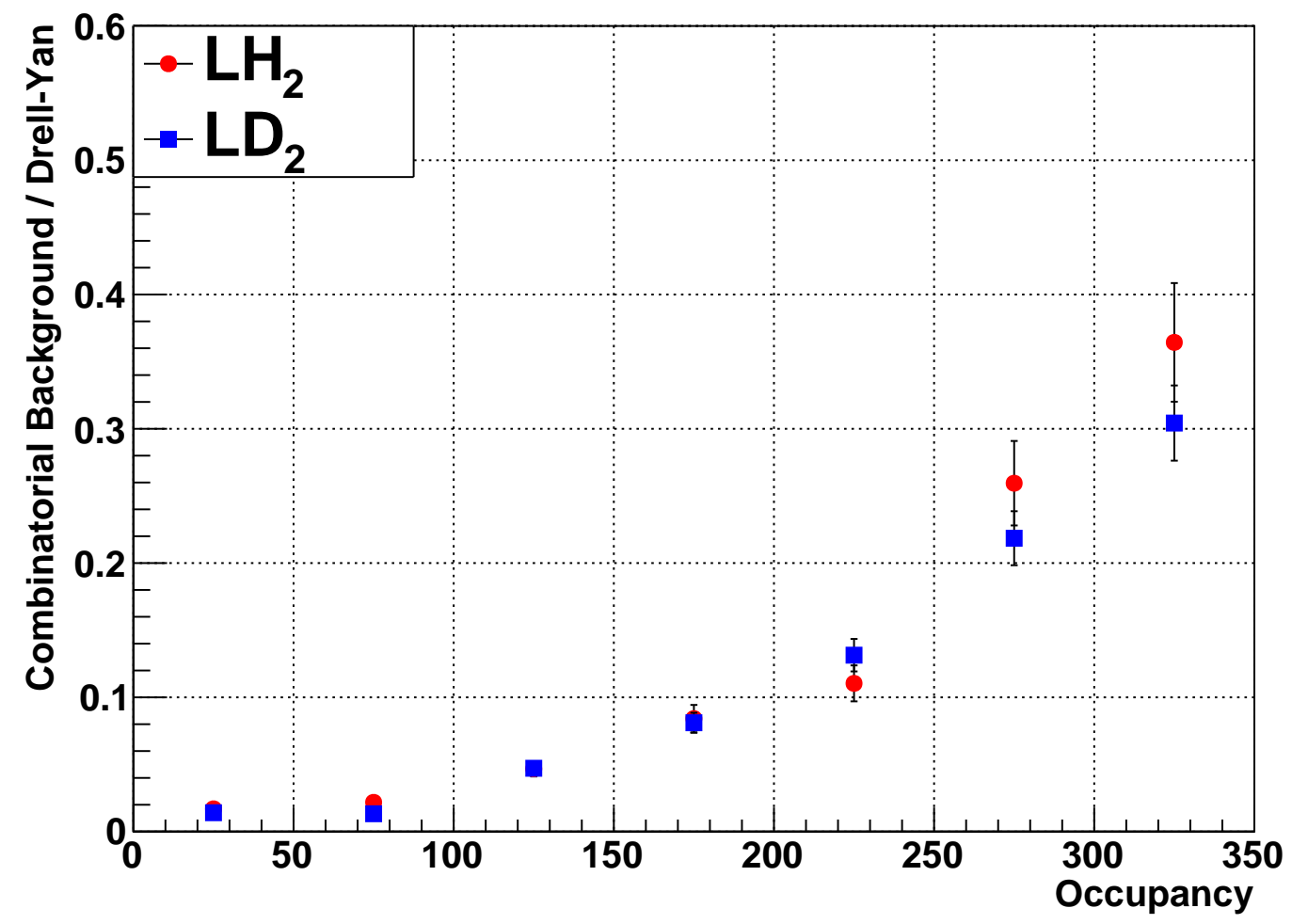

Figure 4.63: Ratio of the combinatorial background to the Drell-Yan dimuons. The red points are the data from the $\mathrm{LH}_{2}$ target and the blue points are the data from the $\mathrm{LD}_{2}$ target. 


\subsection{Cross Section Ratio}

The following variables are used in this section:
$A_{H}:$ Atomic mass
$\lambda_{H}:$ Nuclear interaction length
$\rho_{H}:$ Density
$G_{H}:$ Number of atoms per area
$W_{H}$ : Corrected dimuon yields
$P_{H}:$ Number of beam protons
$L$ : Target Length

The subscript " $H$ " denotes the hydrogen. The variables for the deuterium are denoted with " $D$ ", and those for the empty target are denoted with " $E$ ". The variables are listed in Tab. 4.13.

Table 4.13: Values used in the analysis [44].

\begin{tabular}{cr}
\hline Quantity & Value \\
\hline$A_{H}$ & $1.00794 \mathrm{~g} / \mathrm{mol}$ \\
$A_{D}$ & $2.00140 \mathrm{~g} / \mathrm{mol}$ \\
\hline$\lambda_{H}$ & $52.0 \mathrm{~g} / \mathrm{cm}^{2}$ \\
$\lambda_{D}$ & $71.8 \mathrm{~g} / \mathrm{cm}^{2}$ \\
\hline$\rho_{H}$ & $0.0708 \mathrm{~g} / \mathrm{cm}^{3}$ \\
$\rho_{D}$ & $0.1634 \mathrm{~g} / \mathrm{cm}^{3}$ \\
\hline$G_{H}$ & $2.076 \times 10^{24}$ atoms $/ \mathrm{cm}^{2}$ \\
\hline$P_{H}$ & $2.0790 \times 10^{17} \mathrm{protons}$ \\
$P_{D}$ & $1.0352 \times 10^{17} \mathrm{protons}$ \\
$P_{E}$ & $4.2531 \times 10^{16}$ protons \\
\hline$L$ & $50.8 \mathrm{~cm}$ \\
\hline
\end{tabular}

The $\mathrm{LD}_{2}$ target is not pure deuterium but is contaminated with the hydrogen. The purity of the $\mathrm{LD}_{2}\left(F_{D}\right)$ is defined as the fraction of the deuterium in the $\mathrm{LD}_{2}$ target. The purity in each Roadset is shown in Tab 4.14. As shown in Tab. 4.14, the purity is not the same between the Roadsets. The Table 4.14: The purity of deuterium in $\mathrm{LD}_{2}$ target in each Roadset. The fraction is based on the number of the deuterons and the proton.

\begin{tabular}{cc}
\hline Roadset & Fraction \\
\hline 57 & $0.904_{-0.00}^{+0.05}$ \\
$62-1$ & $0.955_{-0.05}^{+0.00}$ \\
$62-2 \& 67-1$ & $0.912_{-0.01}^{+0.04}$ \\
$67-2 \& 70$ & $1.000_{-0.00}^{+0.00}$ \\
\hline
\end{tabular}


purity for the analysis is averaged purity with number of dimuons on $\mathrm{LD}_{2}$ target:

$$
\left(F_{D}\right)_{\text {avg }} \equiv \sum \frac{\left(F_{D}\right)_{i}}{(n)_{i}}
$$

where $i$ is the Roadset, and $n$ is the number of dimuons in the Roadset $i$. The effective variables are defined to take the contamination into account as follows:

$$
\begin{aligned}
A_{\mathrm{eff}} & =A_{H} \cdot\left(1-F_{D}\right)+A_{D} \cdot F_{D}, \\
\rho_{\mathrm{eff}} & =\rho_{H} \cdot\left(1-F_{D}\right)+\rho_{D} \cdot F_{D}, \\
\frac{1}{\lambda_{\mathrm{eff}}} & =\frac{1-F_{D}}{\lambda_{H}}+\frac{F_{D}}{\lambda_{D}}, \\
\alpha_{D} & =1-\exp \left(-\frac{L \cdot \rho_{\mathrm{eff}}}{\lambda_{\mathrm{eff}}}\right), \\
G_{D} & =\alpha_{D} \cdot \frac{\lambda_{\mathrm{eff}}}{A_{\mathrm{eff}}}=2.335 \times 10^{24} \text { atoms } / \mathrm{cm}^{2},
\end{aligned}
$$

where $\alpha_{D}$ is the interaction fraction and $L$ is the target length.

\subsubsection{Cross Section Ratio Calculation}

The cross sections of $\mathrm{LH}_{2}$ and $\mathrm{LD}_{2}$ targets are

$$
\begin{aligned}
& \sigma_{\mathrm{LH}_{2}}=\sigma_{p p}, \\
& \sigma_{\mathrm{LD}_{2}}=\sigma_{p d} \cdot F_{D}+\sigma_{p p} \cdot\left(1-F_{D}\right),
\end{aligned}
$$

and the cross section ratio is

$$
\frac{\sigma_{p d}}{2 \sigma_{p p}}=\left(\frac{\sigma_{\mathrm{LD}_{2}}}{\sigma_{\mathrm{LH}_{2}}}+F_{D}-1\right) \cdot \frac{1}{2 F_{D}} .
$$

Using measured variables, the cross sections of $\mathrm{LH}_{2}$ and $\mathrm{LD}_{2}$ targets are also expressed as follows:

$$
\begin{aligned}
\sigma_{\mathrm{LH}_{2}} & =\left(\frac{W_{H}}{P_{H}}-\frac{W_{E}}{P_{E}}\right) \cdot \frac{1}{G_{H}}, \\
\sigma_{\mathrm{LD}_{2}} & =\left(\frac{W_{D}}{P_{D}}-\frac{W_{E}}{P_{E}}\right) \cdot \frac{1}{G_{D}} .
\end{aligned}
$$

Therefore,

$$
\frac{\sigma_{p d}}{2 \sigma_{p p}}=\left\{\left(\left(\frac{W_{D}}{P_{D}}-\frac{W_{E}}{P_{E}}\right) \cdot \frac{1}{G_{D}}\right) /\left(\left(\frac{W_{H}}{P_{H}}-\frac{W_{E}}{P_{E}}\right) \cdot \frac{1}{G_{H}}\right)+F_{D}-1\right\} \cdot \frac{1}{2 F_{D}} .
$$

\subsubsection{Error Estimation}

Statistical Error The statistical errors are calculated as follows:

$$
\begin{aligned}
& \delta_{\text {stat }}^{+} \equiv \delta_{\text {stat }}^{\mathrm{LH}_{2}} \oplus \delta_{\text {stat }}^{\mathrm{LD}_{2}} \oplus \delta_{\text {stat }}^{\text {Empty }} \oplus \delta_{K / \mathrm{LH}_{2}}^{+} \oplus \delta_{K / \mathrm{LD}_{2}}^{-}, \\
& \delta_{\text {stat }}^{-} \equiv \delta_{\text {stat }}^{\mathrm{LH}_{2}} \oplus \delta_{\text {stat }}^{\mathrm{LD}_{2}} \oplus \delta_{\text {stat }}^{\text {Empty }} \oplus \delta_{K / \mathrm{LH}_{2}}^{-} \oplus \delta_{K / \mathrm{LD}_{2}}^{+},
\end{aligned}
$$

where $\delta_{\text {stat }}^{\mathrm{LH}_{2}}, \delta_{\text {stat }}^{\mathrm{LD}_{2}}$ and $\delta_{\text {stat }}^{\text {Empty }}$ are the statistical errors propagated from Eq. 4.31, and $\delta_{K / \mathrm{LH}_{2}}^{ \pm}$and $\delta_{K / \mathrm{LD}_{2}}^{ \pm}$are the errors of the tracking efficiency correction. 
Systematic Error The systematic errors are calculated as follows:

$$
\begin{aligned}
& \delta_{\text {syst }}^{+} \equiv \delta_{F_{D}}^{-}+\delta_{R R}^{+}, \\
& \delta_{\text {syst }}^{-} \equiv \delta_{F_{D}}^{+}+\delta_{R R}^{-},
\end{aligned}
$$

$\delta_{F_{D}}^{ \pm}$is the error due to the hydrogen contamination in $\mathrm{LD}_{2}$ target, and $\delta_{R R}^{ \pm}$is the error due to the occupancy dependence.

The $\delta_{R R}^{ \pm}$is calculated based on Fig. 4.65 and Fig. 4.64. Figure 4.64 is the occupancy distribution of dimuons used in the analysis. The average occupancy is $O_{\text {avg }}=139$. Figure 4.65 shows the fitting

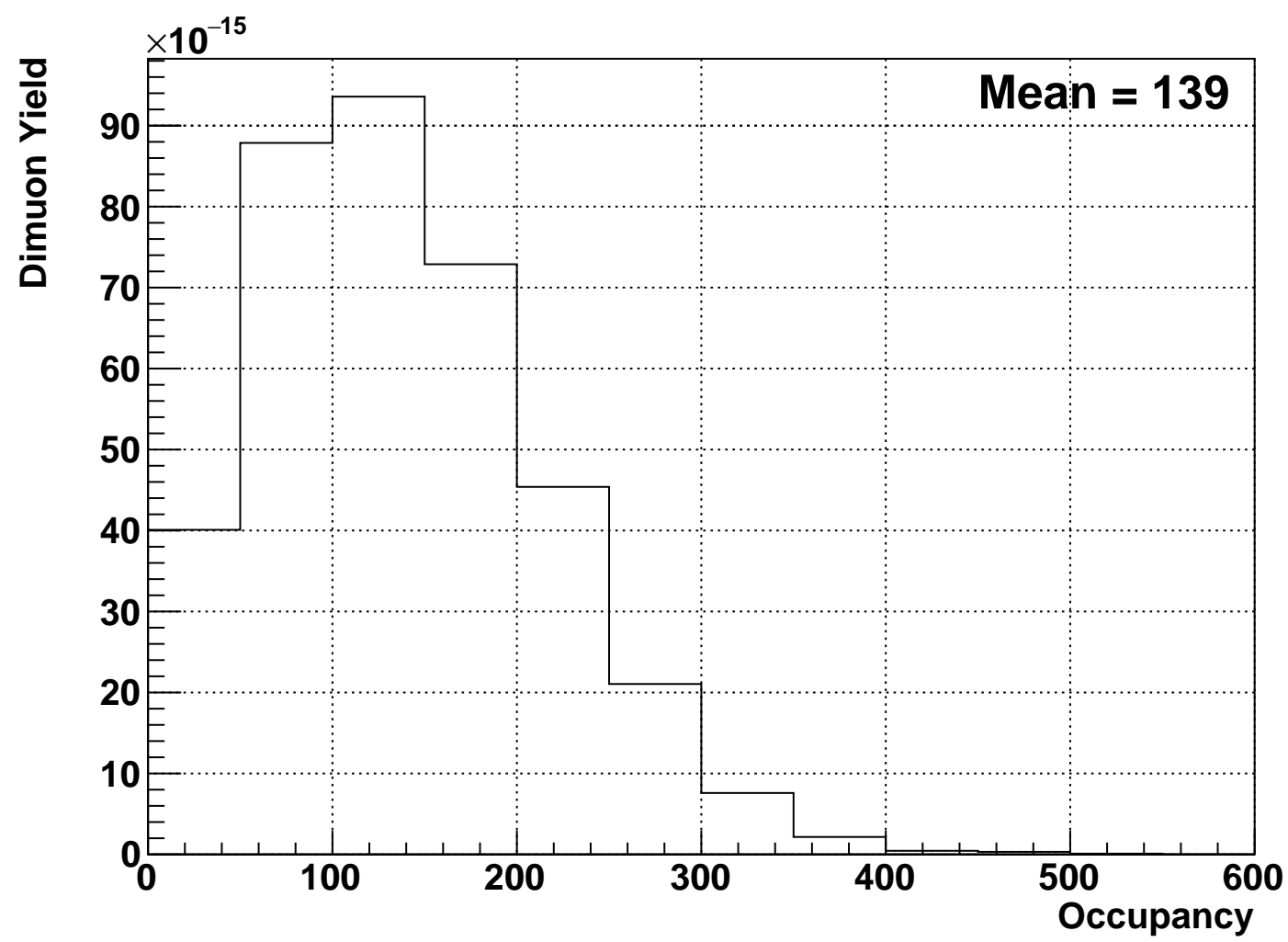

Figure 4.64: Occupancy distribution of dimuons used in the analysis. The average occupancy is 139. The number of the sense wires in the St. 1 drift chamber is 1124.

result of Fig. 4.58, which is the occupancy dependence of the dimuon yield ratio of $\mathrm{LD}_{2}$ to $\mathrm{LH}_{2}$ after the tracking efficiency correction and the empty target subtraction. The fitting function of Fig. 4.65 is:

$$
f(O)=p_{0}+p_{1} O
$$

where $O$ is the occupancy. Table 4.15 shows the fitting result of Fig. 4.65. The slope is consistent with zero. The error of the fitting at average occupancy $\left(O=O_{\text {avg }}=139\right)$ is

$$
\delta=\sqrt{\delta_{p_{0}}^{2}+\left(\delta_{p_{1}} O_{\mathrm{avg}}\right)^{2}+\rho \delta_{p_{0}} \delta_{p_{1}} O_{\mathrm{avg}}}
$$




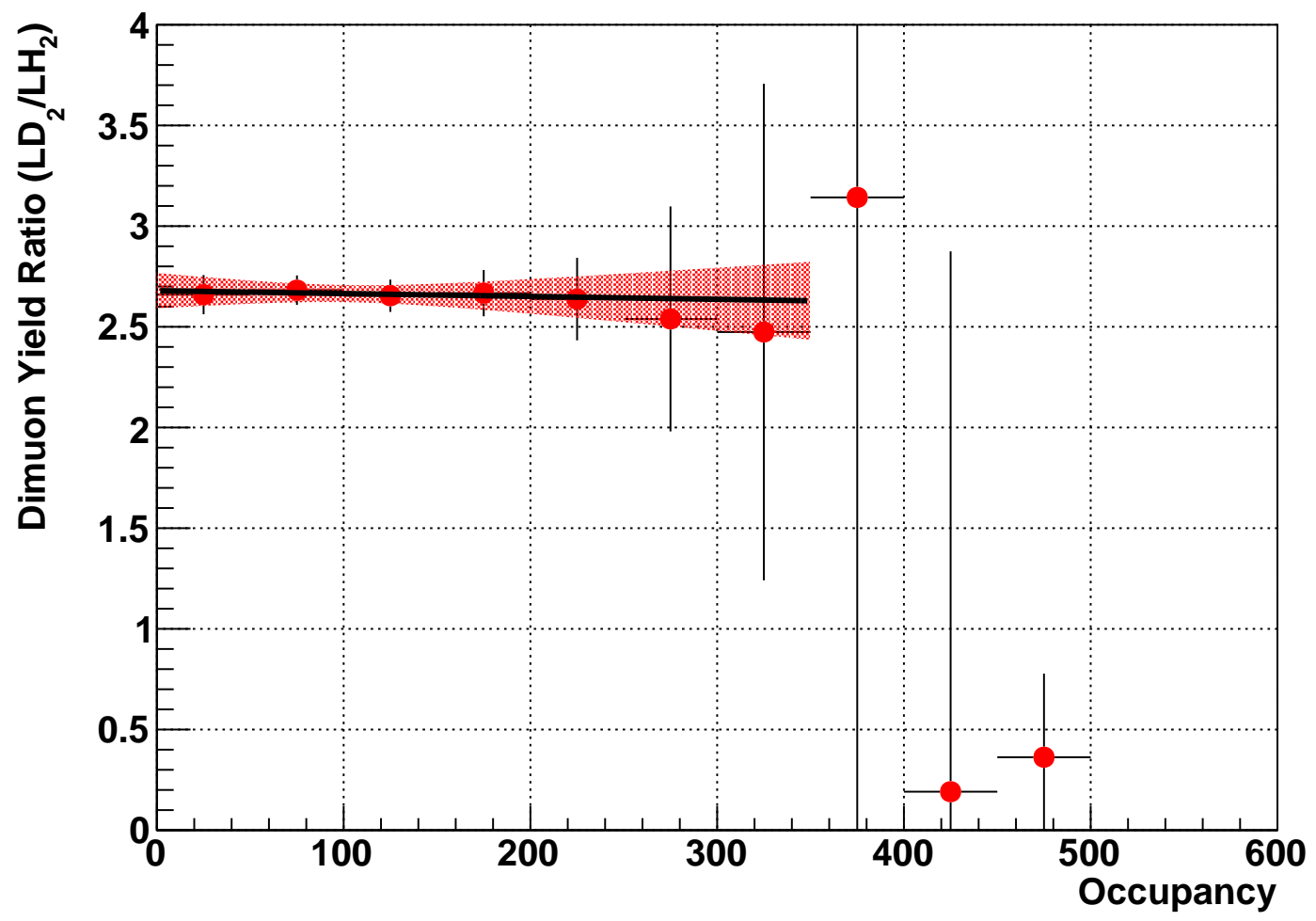

Figure 4.65: Fitting result of occupancy dependence of dimuon yield ratio. It is fitted with a linear function. The red band shows a uncertainty of the fit.

Table 4.15: Fitting result of Fig. 4.65.

\begin{tabular}{ccc}
\hline$p_{0}$ & $p_{1}$ & correlation \\
\hline $2.68 \pm 0.09$ & $-(1.42 \pm 7.60) \times 10^{-4}$ & -0.87 \\
\hline
\end{tabular}


where $\rho$ is the correlation. The estimation of the systematic error due to the occupancy dependence is performed by shifting the dimuon yield ratio by $\pm \delta$ :

$$
\delta_{R R}^{ \pm}=\left\{\left(\left(\frac{W_{D}}{P_{D}}-\frac{W_{E}}{P_{E}}\right) \cdot \frac{1}{G_{D}}\right) /\left(\left(\frac{W_{H}}{P_{H}}-\frac{W_{E}}{P_{E}}\right) \cdot \frac{1}{G_{H}}\right) \pm \delta+F_{D}-1\right\} \cdot \frac{1}{2 F_{D}}-\frac{\sigma_{p d}}{2 \sigma_{p p}} .
$$

The systematic error due to the combinatorial background is estimated using the dimuon yields ratio of $\mathrm{LD}_{2}$ and $\mathrm{LH}_{2}$ by shifting the cut-off mass from $4.2 \mathrm{GeV}$ to $4.5 \mathrm{GeV}$. Figure 4.66 shows the dimuon yield ratio of $\mathrm{LH}_{2}$ and $\mathrm{LD}_{2}$ with the cut-off mass of $4.2 \mathrm{GeV}$ and $4.5 \mathrm{GeV}$. The green points

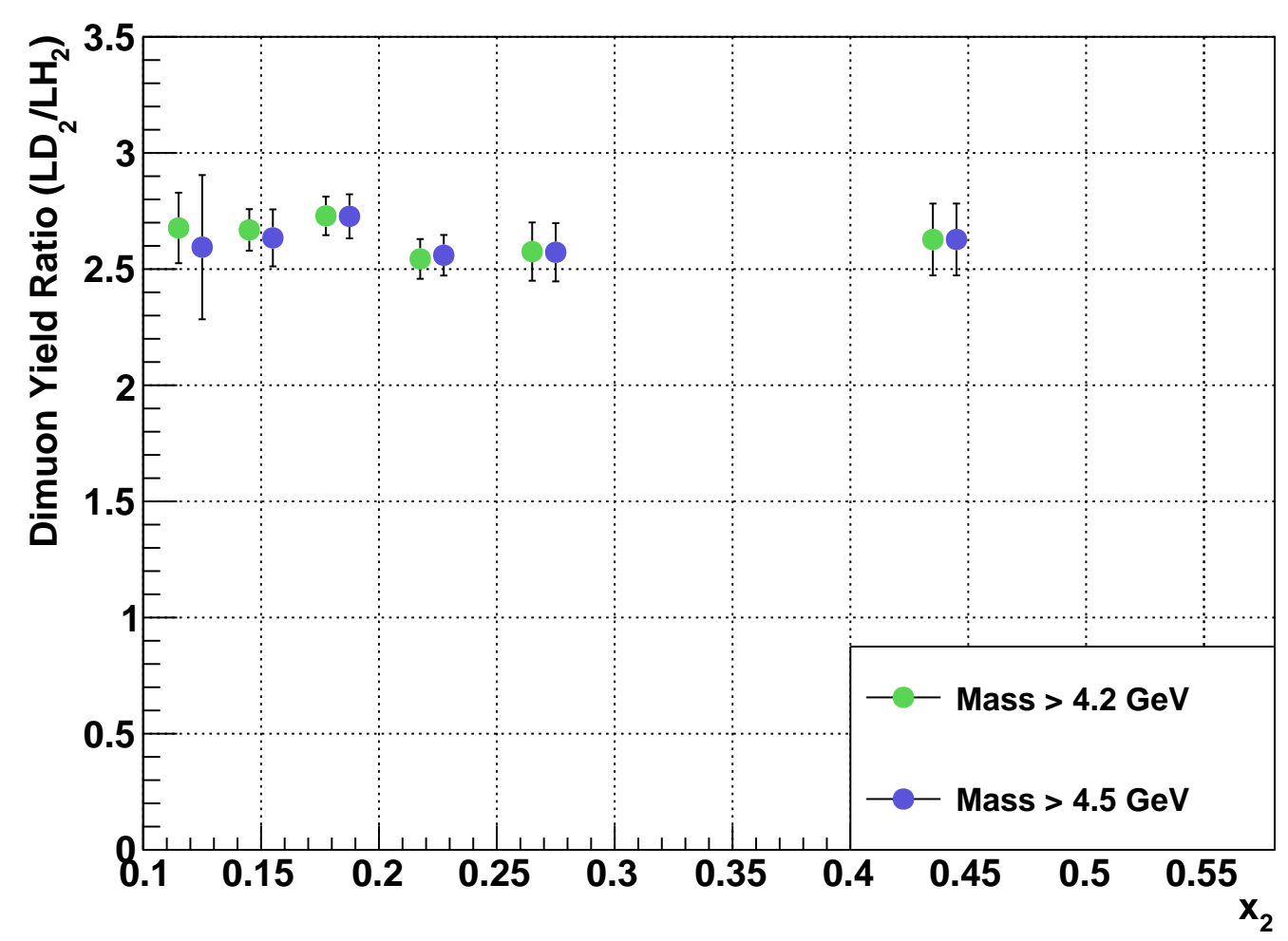

Figure 4.66: Dimuon yield ratio of $\mathrm{LH}_{2}$ and $\mathrm{LD}_{2}$ with cut-off mass of $4.2 \mathrm{GeV}$ and $4.5 \mathrm{GeV}$. The green points are the dimuon yield ratio with a cut-off mass of $4.2 \mathrm{GeV}$. The blue points are the dimuon yield ratio with a cut-off mass of $4.5 \mathrm{GeV}$.

are the dimuon yield ratio with a cut-off mass of $4.2 \mathrm{GeV}$. The blue points are the dimuon yield ratio with a cut-off mass of $4.5 \mathrm{GeV}$. The difference is small enough. The error due to the background is negligible.

The systematic errors due to the detector acceptance, the detector efficiency, the trigger efficiency, and the intensity of the beam do not have to be considered because they are cancelled out by taking the ratio.

The sources of the systematic errors are summarized in Tab. 4.16. 
Table 4.16: Sources of systematic errors.

\begin{tabular}{ll}
\hline Source & Error $(\%)$ \\
\hline Tracker efficiency correction & (included into statistical error) \\
Empty target correction & (included into statistical error) \\
Remaining occupancy dependence & $\pm 1 \%$ \\
& $+\sim 2.0 \%$ \\
Target contamination & $-\sim 0.4 \%$ \\
& negligible \\
Combinatorial background & negligible \\
Detector acceptance & negligible \\
Detector efficiency & negligible \\
Trigger efficiency & \\
\hline
\end{tabular}

Table 4.17: Cross section ratio and statistical and systematic errors at each $x_{2}$ bin. The average $x_{2}$ in each bin is also listed.

\begin{tabular}{cc|c|ccccc}
\hline$x_{2} \min$ & $x_{2} \max$ & $x_{2}$ average & $\sigma_{p d} / 2 \sigma_{p p}$ & $\delta_{\text {stat }}^{+}$ & $\delta_{\text {stat }}^{-}$ & $\delta_{\text {syst }}^{+}$ & $\delta_{\text {syst }}^{-}$ \\
\hline 0.10 & 0.13 & 0.1199 & 1.2665 & 0.0689 & 0.0687 & 0.0154 & 0.0401 \\
0.13 & 0.16 & 0.1459 & 1.2136 & 0.0535 & 0.0528 & 0.0151 & 0.0384 \\
0.16 & 0.20 & 0.1766 & 1.2478 & 0.0413 & 0.0411 & 0.0153 & 0.0395 \\
0.20 & 0.24 & 0.2149 & 1.1667 & 0.0412 & 0.0410 & 0.0149 & 0.0368 \\
0.24 & 0.29 & 0.2623 & 1.1950 & 0.0546 & 0.0545 & 0.0151 & 0.0378 \\
0.29 & 0.35 & 0.3148 & 1.2145 & 0.0824 & 0.0821 & 0.0152 & 0.0384 \\
0.35 & 0.45 & 0.3850 & 1.4071 & 0.1772 & 0.1769 & 0.0160 & 0.0449 \\
0.45 & 0.58 & 0.4899 & 1.0099 & 0.2372 & 0.2368 & 0.0142 & 0.0315 \\
\hline
\end{tabular}

\subsubsection{Results}

Figure 4.67 shows the cross section ratio as a function of $x_{2}$. The cross section ratio is larger than one at $0.1<x_{2}<0.45$. At the highest bin $\left(0.45<x_{2}<0.58\right)$, the cross section ratio is consistent with unity within the error.

Table 4.17 shows the cross section ratio at each bin. The breakdown of the errors is shown in Tab. 4.18 and Tab. 4.19. 


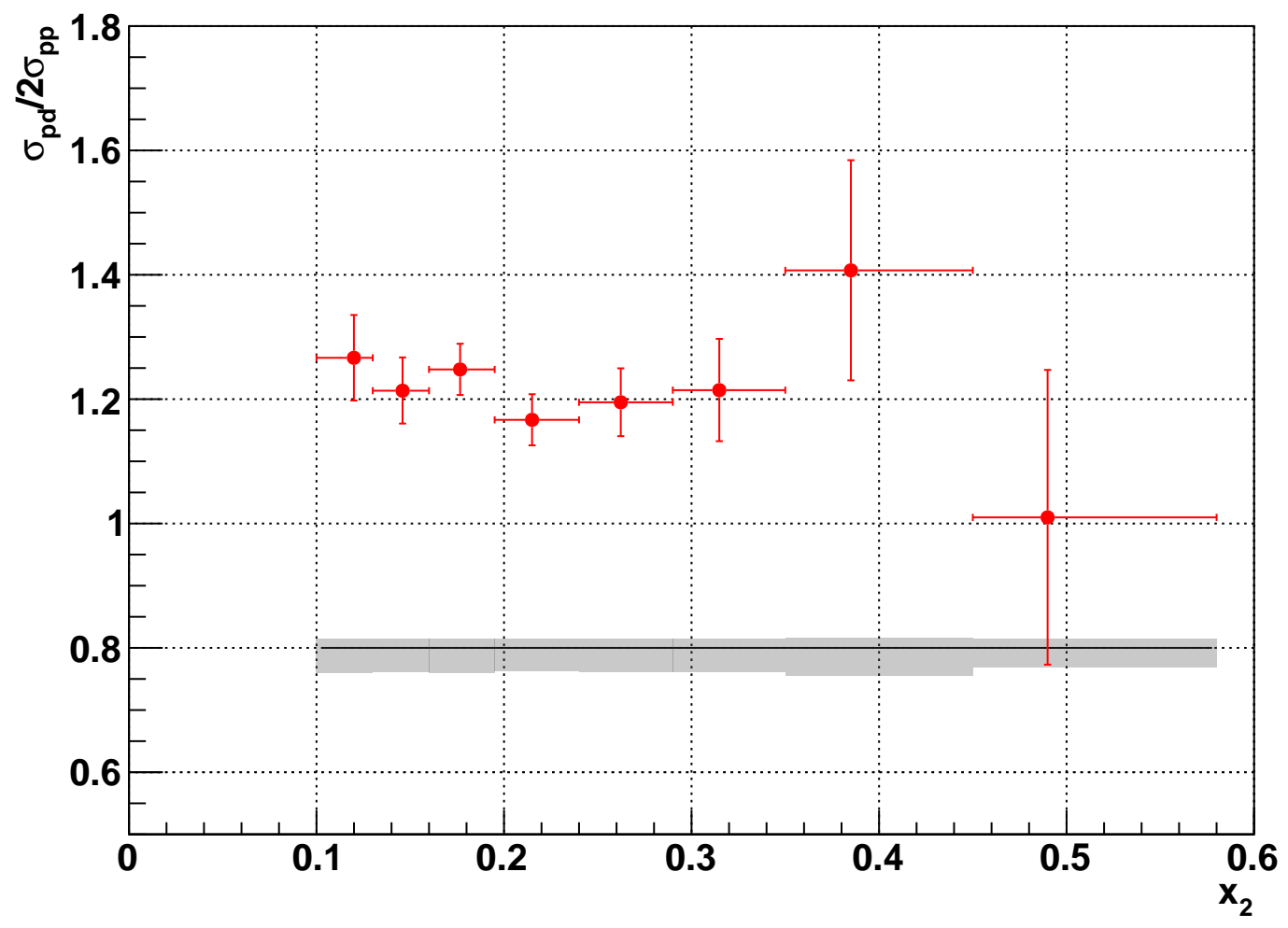

Figure 4.67: Cross section ratio as a function of $x_{2}$. The gray block shows the systematic error.

Table 4.18: Breakdown of errors with $+1 \sigma$ shift.

\begin{tabular}{cc|cccccc}
\hline$x_{2} \min$ & $x_{2} \mathrm{max}$ & \multicolumn{1}{|c}{$\delta_{\text {stat }}^{\mathrm{LH}_{2} \& \mathrm{LD}_{2} \& E m p t y}$} & $\delta_{K / \mathrm{LH}_{2}}^{+}$ & $\delta_{K / \mathrm{LD}_{2}}^{+}$ & $\delta_{K / \text { Empty }}^{+}$ & $\delta_{F_{D}}^{+}$ & $\delta_{R R}^{+}$ \\
\hline 0.10 & 0.13 & 0.0629 & 0.0200 & -0.0188 & -0.0030 & -0.0253 & 0.0104 \\
0.13 & 0.16 & 0.0358 & 0.0278 & -0.0263 & -0.0040 & -0.0236 & 0.0105 \\
0.16 & 0.20 & 0.0335 & 0.0174 & -0.0162 & -0.0023 & -0.0247 & 0.0105 \\
0.20 & 0.24 & 0.0348 & 0.0159 & -0.0147 & -0.0019 & -0.0221 & 0.0106 \\
0.24 & 0.29 & 0.0495 & 0.0169 & -0.0150 & -0.0019 & -0.0230 & 0.0105 \\
0.29 & 0.35 & 0.0739 & 0.0258 & -0.0241 & -0.0036 & -0.0236 & 0.0105 \\
0.35 & 0.45 & 0.1677 & 0.0444 & -0.0316 & -0.0107 & -0.0297 & 0.0102 \\
0.45 & 0.58 & 0.2326 & 0.0310 & -0.0300 & -0.0082 & -0.0171 & 0.0109 \\
\hline
\end{tabular}


Table 4.19: Breakdown of errors with $-1 \sigma$ shift.

\begin{tabular}{cc|cccccc}
\hline$x_{2} \min$ & $x_{2} \max$ & $\delta_{\text {stat }}^{\mathrm{LH}_{2} \& \mathrm{LD}_{2} \& E m p t y}$ & $\delta_{K / \mathrm{LH}_{2}}^{-}$ & $\delta_{K / \mathrm{LD}_{2}}^{-}$ & $\delta_{K / \text { Empty }}^{-}$ & $\delta_{F_{D}}^{-}$ & $\delta_{R R}^{-}$ \\
\hline 0.10 & 0.13 & 0.0629 & -0.0201 & 0.0196 & 0.0031 & 0.0050 & -0.0149 \\
0.13 & 0.16 & 0.0358 & -0.0283 & 0.0282 & 0.0044 & 0.0046 & -0.0148 \\
0.16 & 0.20 & 0.0335 & -0.0174 & 0.0167 & 0.0024 & 0.0048 & -0.0149 \\
0.20 & 0.24 & 0.0348 & -0.0160 & 0.0151 & 0.0019 & 0.0043 & -0.0147 \\
0.24 & 0.29 & 0.0495 & -0.0169 & 0.0154 & 0.0019 & 0.0045 & -0.0148 \\
0.29 & 0.35 & 0.0739 & -0.0260 & 0.0255 & 0.0038 & 0.0046 & -0.0148 \\
0.35 & 0.45 & 0.1677 & -0.0452 & 0.0338 & 0.0119 & 0.0058 & -0.0151 \\
0.45 & 0.58 & 0.2326 & -0.0315 & 0.0325 & 0.0100 & 0.0034 & -0.0144 \\
\hline
\end{tabular}




\section{Chapter 5}

\section{Extraction of $\bar{d} / \bar{u}$ Ratio}

The antiquark flavor asymmetry is approximately related to the cross section ratio $\sigma_{p d} / 2 \sigma_{p d}$ :

$$
\frac{\sigma_{p d}}{2 \sigma_{p p}} \approx \frac{1}{2}\left(1+\frac{\bar{d}}{\bar{u}}\right) .
$$

However, Eq. (5.1) is not appropriate to derive $\bar{d} / \bar{u}$ precisely because it is calculated based on several assumptions. For extracting $\bar{d} / \bar{u}$ precisely and reliably, an iterative analysis has been performed at each $x_{2}$ bin. The cross section is a function of $x_{1}$ and $x_{2}$ and is expressed in Eq. (2.11). The flow chart of this analysis is shown in Fig. 5.1. In the present analysis, the new method for tracking efficiency correction based on the chamber occupancy is adopted as described in the previous chapter. As the result, almost all the available data become analyzable. So, the analyzable data increased nearby $40 \%$ in the present analysis compared to the earlier analysis. The $R_{\text {data }}$ is the cross section ratio in data. It is derived with Eq. (4.31) and is shown in Fig. 4.67 and Tab. 4.17. 




Figure 5.1: Flow chart of the iterative analysis for extracting $\bar{d} / \bar{u}$. 


\section{$5.1 R_{\text {pred }}$ Calculation}

The $R_{\text {pred }}$ is the predicted cross section ratio in the leading order calculation using the estimate of $\bar{d} / \bar{u}$ and the CT10LO PDF set [45] provided by the CTEQ group. The cross sections of $\sigma_{p p}, \sigma_{p n}$ and $\sigma_{p d}$ are written as follows:

$$
\begin{aligned}
& \sigma_{p p} \propto 4 u\left(x_{1}\right) \bar{u}\left(x_{2}\right)+4 \bar{u}\left(x_{1}\right) u\left(x_{2}\right)+d\left(x_{1}\right) \bar{d}\left(x_{2}\right)+\bar{d}\left(x_{1}\right) d\left(x_{2}\right)+8 c\left(x_{1}\right) c\left(x_{2}\right)+2 s\left(x_{1}\right) s\left(x_{2}\right), \\
& \sigma_{p n} \propto 4 u\left(x_{1}\right) \bar{d}\left(x_{2}\right)+4 \bar{u}\left(x_{1}\right) d\left(x_{2}\right)+d\left(x_{1}\right) \bar{u}\left(x_{2}\right)+\bar{d}\left(x_{1}\right) u\left(x_{2}\right)+8 c\left(x_{1}\right) c\left(x_{2}\right)+2 s\left(x_{1}\right) s\left(x_{2}\right), \\
& \sigma_{p d}=\sigma_{p p}+\sigma_{p n} .
\end{aligned}
$$

Here, the following are assumed.

- The proton and the neutron are isospin symmetric $\left(u_{p}(x)=d_{n}(x), d_{p}(x)=u_{n}(x), \bar{u}_{p}(x)=\right.$ $\left.\bar{d}_{n}(x), \bar{d}_{p}(x)=\bar{u}_{n}(x)\right)$.

- The nuclear effect is ignored $\left(\sigma_{p d}=\sigma_{p p}+\sigma_{p n}\right)$.

- The $c$ in the proton is the same as $\bar{c}$.

- The $s$ in the proton is the same as $\bar{s}$.

- The heavy quarks, such as top and bottom quarks, are ignored.

The validity of assuming the isospin symmetry can be explained with the results of the global analyses of different reactions. Many groups performed the global analysis and provided the consistent results under the assumption of the isospin symmetry. Therefore, the isospin symmetry is adopted in the present analysis. The values of $u(x), d(x), s(x), c(x)$ and $\bar{u}(x)+\bar{d}(x)$ are taken from the PDF set. $\bar{u}$ and $\bar{d}$ are calculated as follows:

$$
\begin{aligned}
& \bar{u}(x)=\frac{[\bar{u}(x)+\bar{d}(x)]_{\mathrm{PDF}}}{\left[\frac{\bar{d}(x)}{\bar{u}(x)}\right]_{\mathrm{EST}}+1} \\
& \bar{d}(x)=\left[\frac{\bar{d}(x)}{\bar{u}(x)}\right]_{\mathrm{EST}} \cdot \bar{u}(x),
\end{aligned}
$$

where $[\bar{u}(x)+\bar{d}(x)]_{\mathrm{PDF}}$ is $\bar{u}(x)+\bar{d}(x)$ taken from PDF set, and $[\bar{d} / \bar{u}]_{\mathrm{EST}}$ is the estimate of $\bar{d} / \bar{u}$. The $\bar{d} / \bar{u}$ is assumed to be unity at the first iteration. The estimate of $\bar{d} / \bar{u}$ at $i$ th iteration in $0.1<x<0.58$ is taken from the result of $(i-1)$ th iteration. Although the iterative analysis is performed in each $x_{2}$ bin, $\bar{d} / \bar{u}$ at $x_{2}<0.1$ or $x_{2}>0.58$ is also needed since $\bar{d}\left(x_{1}\right)$ and $\bar{u}\left(x_{1}\right)$ are used for the calculation of $R_{\text {pred. }}$. The $\bar{d} / \bar{u}$ at $x<0.1$ or $x>0.58$ is taken from PDF set. The $R_{\text {pred }}=\sigma_{p d} / 2 \sigma_{p p}$ is calculated with mass, $x_{1}$ and $x_{2}$ event-by-event of the real data. Thus, the number of obtained $R_{\text {pred }}$ is the number of events in the real data. Then, the average of $R_{\text {pred }}$ is used as $R_{\text {pred }}: R_{\text {pred }}=\left\langle R_{\text {pred }}\right\rangle$.

\subsection{Update $\bar{d} / \bar{u}$}

The $\bar{d} / \bar{u}$ is approximately proportional to the cross section ratio as shown in Eq. (5.1). The $\bar{d} / \bar{u}$ is updated with $\bar{d} / \bar{u}_{\text {new }}=\bar{d} / \bar{u}_{\text {old }}+\Delta$, where $\Delta=R_{\text {data }}-R_{\text {pred. }}$ The next iteration is performed with this updated $\bar{d} / \bar{u}$. The iterative analysis is repeated until $|\Delta|$ satisfies $|\Delta|<10^{-4}$. 


\subsection{Error Estimation}

\subsubsection{Statistical Error}

In the $R_{\text {pred }}$ calculation in a $x_{2}$ bin, $\bar{d} / \bar{u}$ at other $x_{2}$ bins are also used according to the section 5.1 . Therefore, the statistical error on a $x_{2}$ bin includes the statistical errors on other $x_{2}$ bins. The statistical error at $i$ th bin is calculated as

$$
\delta_{i}=\sqrt{\sum_{j} r_{i j}^{2}}
$$

where $r_{i j}$ is a component of the statistical error. The procedure of $r_{i j}$ calculation is as follows:

1. Shift the cross section ratio at $j$ bin by its statistical error upward.

2. Calculate $\bar{d} / \bar{u}\left(=\bar{d} / \bar{u}_{i j}^{+}\right)$at $i$ bin.

3. Calculate $r_{i j}^{+}=\bar{d} / \bar{u}_{i j}^{+}-\bar{d} / \bar{u}_{\text {original }}$.

4. Shift the cross section ratio at $j$ bin by its statistical error downward.

5. Calculate $\bar{d} / \bar{u}\left(=\bar{d} / \bar{u}_{i j}^{-}\right)$at $i$ bin.

6. Calculate $r_{i j}^{-}=\bar{d} / \bar{u}_{\text {original }}-\bar{d} / \bar{u}_{i j}^{-}$.

7. $r_{i j}=\left(r_{i j}^{+}+r_{i j}^{-}\right) / 2$.

Table 5.1 and Tab. 5.2 shows $r_{i j}^{+}$and $r_{i j}^{-}$, respectively. The average of them are listed in Tab. 5.3.

Table 5.1: Statistical error matrix at each $i$ bin by upward shifting $j$ bin.

\begin{tabular}{c|cccccccc}
\hline & $i$ & & & & & & & \\
$j$ & 0 & 1 & 2 & 3 & 4 & 5 & 6 & 7 \\
\cline { 2 - 9 } & 0.173186 & 0.000213 & 0.000000 & 0.000000 & 0.000000 & 0.000000 & 0.000000 & 0.000000 \\
1 & 0.000000 & 0.133128 & 0.000160 & 0.000000 & 0.000000 & 0.000009 & 0.000084 & 0.001941 \\
2 & 0.000001 & 0.000001 & 0.108461 & 0.000200 & 0.000001 & 0.000048 & 0.000279 & 0.010168 \\
3 & 0.000001 & 0.000001 & 0.000001 & 0.109444 & 0.000052 & 0.000068 & 0.000508 & 0.014379 \\
4 & 0.000001 & 0.000001 & 0.000001 & 0.000001 & 0.157780 & 0.000093 & 0.002970 & 0.025718 \\
5 & 0.000007 & 0.000007 & 0.000007 & 0.000007 & 0.000007 & 0.272443 & 0.008489 & 0.048748 \\
6 & 0.000448 & 0.000470 & 0.000470 & 0.000470 & 0.000470 & 0.000574 & 0.963803 & 0.203208 \\
7 & 0.000067 & 0.000071 & 0.000071 & 0.000071 & 0.000071 & 0.003651 & 0.032954 & 1.121423 \\
\hline
\end{tabular}

\subsubsection{Systematic Error}

The systematic errors are calculated as follows:

$$
\begin{aligned}
& \delta_{\text {syst }}^{+}=\delta_{\mathrm{CSR}}^{+}+\left|\delta_{\text {high }-x}^{-}\right|, \\
& \delta_{\text {syst }}^{-}=\delta_{\mathrm{CSR}}^{-}+\left|\delta_{\text {high }-x}^{+}\right|,
\end{aligned}
$$


Table 5.2: Statistical error matrix at each $i$ bin by downward shifting $j$ bin.

\begin{tabular}{c|cccccccc}
\hline & $i$ & & & & & & & \\
$j$ & 0 & 1 & 2 & 3 & 4 & 5 & 6 & 7 \\
\cline { 2 - 9 } & 0.169576 & 0.000000 & 0.000000 & 0.000000 & 0.000000 & 0.000000 & 0.000000 & 0.000000 \\
1 & 0.000000 & 0.130894 & 0.000000 & 0.000000 & 0.000000 & 0.000008 & 0.000089 & 0.003521 \\
2 & 0.000001 & 0.000001 & 0.106762 & 0.000001 & 0.000001 & 0.000044 & 0.000258 & 0.018552 \\
3 & 0.000001 & 0.000001 & 0.000001 & 0.107473 & 0.000001 & 0.000064 & 0.000769 & 0.026222 \\
4 & 0.000001 & 0.000001 & 0.000001 & 0.000001 & 0.153117 & 0.000119 & 0.003887 & 0.046574 \\
5 & 0.000007 & 0.000007 & 0.000007 & 0.000007 & 0.000007 & 0.256856 & 0.010594 & 0.087597 \\
6 & 0.000469 & 0.000470 & 0.000470 & 0.000470 & 0.000470 & 0.000449 & 0.794821 & 0.370812 \\
7 & 0.000071 & 0.000071 & 0.000071 & 0.000071 & 0.000071 & 0.003507 & 0.036036 & 0.892715 \\
\hline
\end{tabular}

Table 5.3: Statistical error matrix at each $i$ bin by shifting $j$ bin.

\begin{tabular}{c|cccccccc}
\hline & $i$ & & & & & & & \\
$j$ & 0 & 1 & 2 & 3 & 4 & 5 & 6 & 7 \\
\cline { 2 - 9 } & 0.171381 & 0.000106 & 0.000000 & 0.000000 & 0.000000 & 0.000000 & 0.000000 & 0.000000 \\
1 & 0.000000 & 0.132011 & 0.000080 & 0.000000 & 0.000000 & 0.000009 & 0.000086 & 0.002731 \\
2 & 0.000001 & 0.000001 & 0.107612 & 0.000101 & 0.000001 & 0.000046 & 0.000269 & 0.014360 \\
3 & 0.000001 & 0.000001 & 0.000001 & 0.108458 & 0.000027 & 0.000066 & 0.000638 & 0.020301 \\
4 & 0.000001 & 0.000001 & 0.000001 & 0.000001 & 0.155448 & 0.000106 & 0.003428 & 0.036146 \\
5 & 0.000007 & 0.000007 & 0.000007 & 0.000007 & 0.000007 & 0.264649 & 0.009542 & 0.068173 \\
6 & 0.000459 & 0.000470 & 0.000470 & 0.000470 & 0.000470 & 0.000511 & 0.879312 & 0.287010 \\
7 & 0.000069 & 0.000071 & 0.000071 & 0.000071 & 0.000071 & 0.003579 & 0.034495 & 1.007069 \\
\hline
\end{tabular}


where $\delta_{\mathrm{CSR}}^{ \pm}$is the error propagated to $\bar{d} / \bar{u}$ by shifting cross section ratio by systematic error sizes upward or downward, and $\delta_{\text {high-x }}^{ \pm}$is the error due to the $\bar{d} / \bar{u}$ variance above $x_{2}=0.58$. In the iterative analysis, $\bar{d} / \bar{u}$ at $x<0.1$ or $x>0.58$ is taken from PDF set. At lower $x$ region $(x<0.1), \bar{d} / \bar{u}$ is well known by other experiments, but for $\bar{d} / \bar{u}$ at high $x(x>0.58)$ there is no experimental result. The $\bar{d} / \bar{u}$ at high $x$ is set to excessive values ( 0.5 for downward and 2.0 for upward) to perform the iterative analysis to take the effect due to the $\bar{d} / \bar{u}$ variance above $x_{2}=0.58$ into account.

The errors are summarized in Tab. 5.4.

Table 5.4: Breakdown of $\bar{d} / \bar{u}$ systematic errors.

\begin{tabular}{c|cccc}
\hline$x_{2}$ avg & $\delta_{\text {CSR }}^{+}$ & $\delta_{\text {CSR }}^{-}$ & $\delta_{\text {high }-x}^{+}$ & $\delta_{\text {high }-x}^{-}$ \\
\hline 0.1199 & 0.0385 & 0.0995 & -0.0044 & -0.0005 \\
0.1459 & 0.0376 & 0.0945 & -0.0095 & 0.0004 \\
0.1766 & 0.0394 & 0.1011 & -0.0098 & 0.0005 \\
0.2149 & 0.0384 & 0.0941 & -0.0095 & 0.0004 \\
0.2623 & 0.0408 & 0.1018 & -0.0153 & 0.0005 \\
0.3148 & 0.0443 & 0.1119 & -0.0246 & 0.0005 \\
0.3850 & 0.0633 & 0.1834 & -0.1030 & -0.0003 \\
0.4899 & 0.0565 & 0.1213 & -0.0692 & -0.0032 \\
\hline
\end{tabular}




\subsection{Results}

Figure 5.2 and Tab. 5.5 show the results of $\bar{d} / \bar{u}$ and error calculation. The $\bar{d} / \bar{u}$ is larger than one at



Figure 5.2: The result of the ratio $\bar{d} / \bar{u}$. The gray block shows the systematic error.

each $x_{2}$ in the range of $0.1<x<0.45$. At the highest bin, the $\bar{d} / \bar{u}$ is consistent with one. 
Table 5.5: $\bar{d} / \bar{u}$ and errors.

\begin{tabular}{c|ccccc}
\hline$x_{2}$ avg & $\bar{d} / \bar{u}$ & $\delta_{\text {stat }}^{+}$ & $\delta_{\text {stat }}^{-}$ & $\delta_{\text {syst }}^{+}$ & $\delta_{\text {syst }}^{-}$ \\
\hline 0.1199 & 1.6413 & 0.1732 & 0.1696 & 0.0389 & 0.1039 \\
0.1459 & 1.5209 & 0.1331 & 0.1309 & 0.0380 & 0.1040 \\
0.1766 & 1.6323 & 0.1089 & 0.1084 & 0.0399 & 0.1109 \\
0.2149 & 1.4472 & 0.1104 & 0.1106 & 0.0389 & 0.1037 \\
0.2623 & 1.5650 & 0.1599 & 0.1601 & 0.0413 & 0.1171 \\
0.3148 & 1.7039 & 0.2769 & 0.2716 & 0.0448 & 0.1365 \\
0.3850 & 2.8091 & 0.9850 & 0.8771 & 0.0636 & 0.2864 \\
0.4899 & 1.4171 & 1.1219 & 0.8934 & 0.0597 & 0.1905 \\
\hline
\end{tabular}




\section{Chapter 6}

\section{Discussion on the Result}

\subsection{Comparison with the Previous Results}

Result from the earlier analysis of the SeaQuest was shown in the doctor thesis by Miyasaka, Tokyo Tech, 2016 [46]. The results of the present thesis are compared with those by Miyasaka. The differences of the analyses are

- Raw data set: this thesis uses 3\% more data than Miyasaka

- The present thesis: Roadset 57, 62, 67, 70

- Miyasaka: Roadset 57, 62, 67

- Beam Intensity Corrections: a new method based on the chamber occupancy is used in this thesis.

- The present thesis: Corrections for tracking efficiency based on the occupancy of the St. 1 drift chamber was adopted. The data with the St. 1 occupancy from 0 to 350 are used. As the result, nearby all the available data became analyzable.

- Miyasaka: Corrections based on the chamber intensity (weighted average of the beam intensity to take the effects of other RF-bucket intensity into account). The data with chamber intensity from 40,000 to 60,000 were not used bacause the dimuon yield ratio in this region is significantly lower than the region 0-40,000 after the correction. About $30 \%$ of the dimuons were not used due to this.

As the result, the amount of analyzable data increased nearby $40 \%$ in the present analysis compared to the earlier analysis. The comparison plots are shown in Fig. 6.1. In both of the cross section ratio and $\bar{d} / \bar{u}$, the two results are in good agreement at lower four data points. The differences can be seen mainly at higher three data points.

The correction method in this thesis is performed based on the chamber occupancy. The advantage of the occupancy-based correction is that

- the correction reflects the real chamber performance, and

- the tracking efficiency of empty target is also clear as well as the target data.

Thus, the correction method used in this thesis is more reliable and precise. 


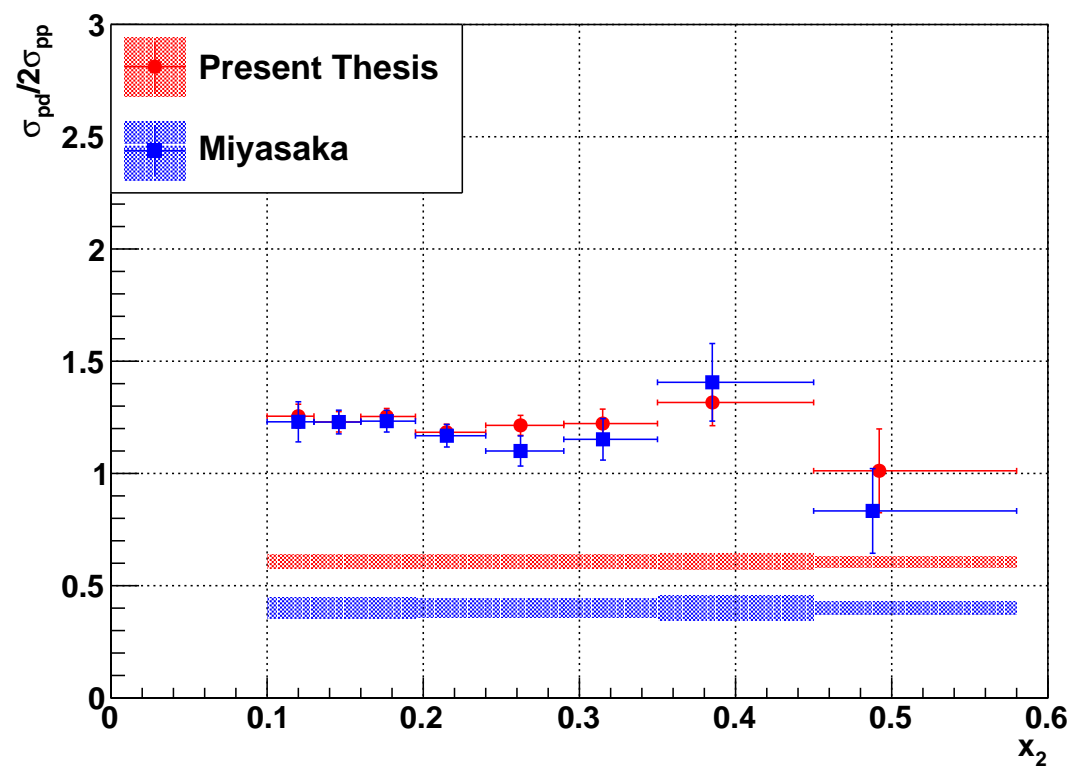

(a) Cross section ratio

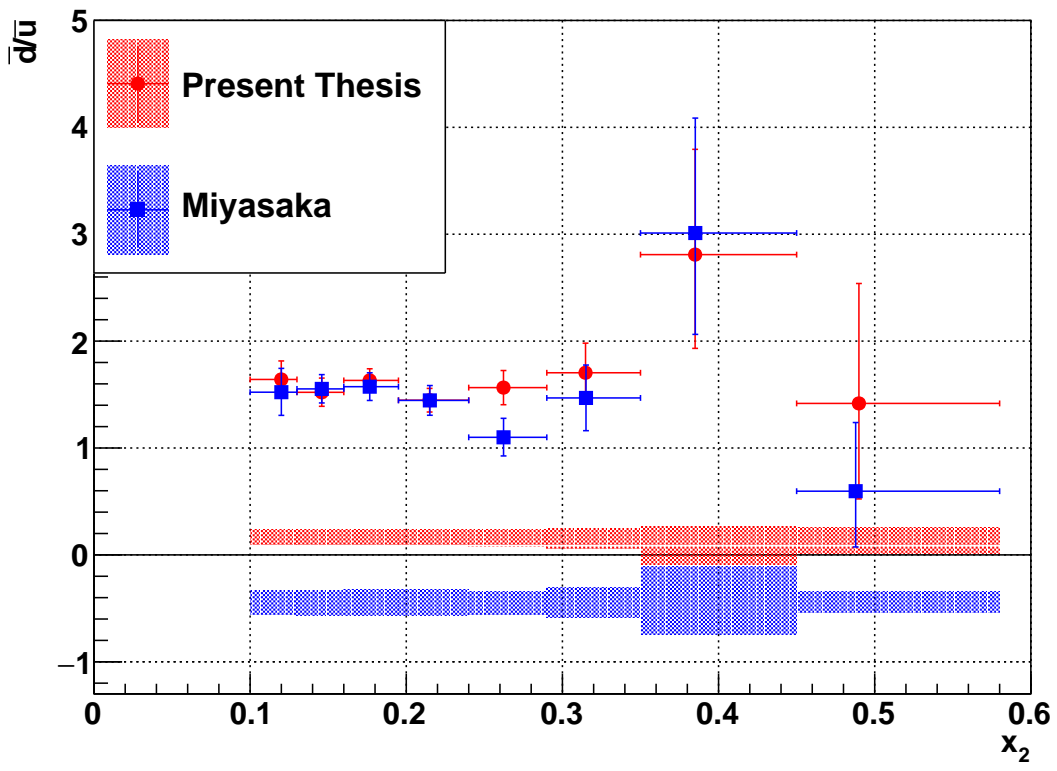

(b) Flavor asymmetry

Figure 6.1: Comparison of results in present thesis and those by Miyasaka. 


\subsection{Comparison with E866 Experiment}

Figure 6.2 shows the comparison with the E866 result. The results at the lower $x$ regions of $0.1<$

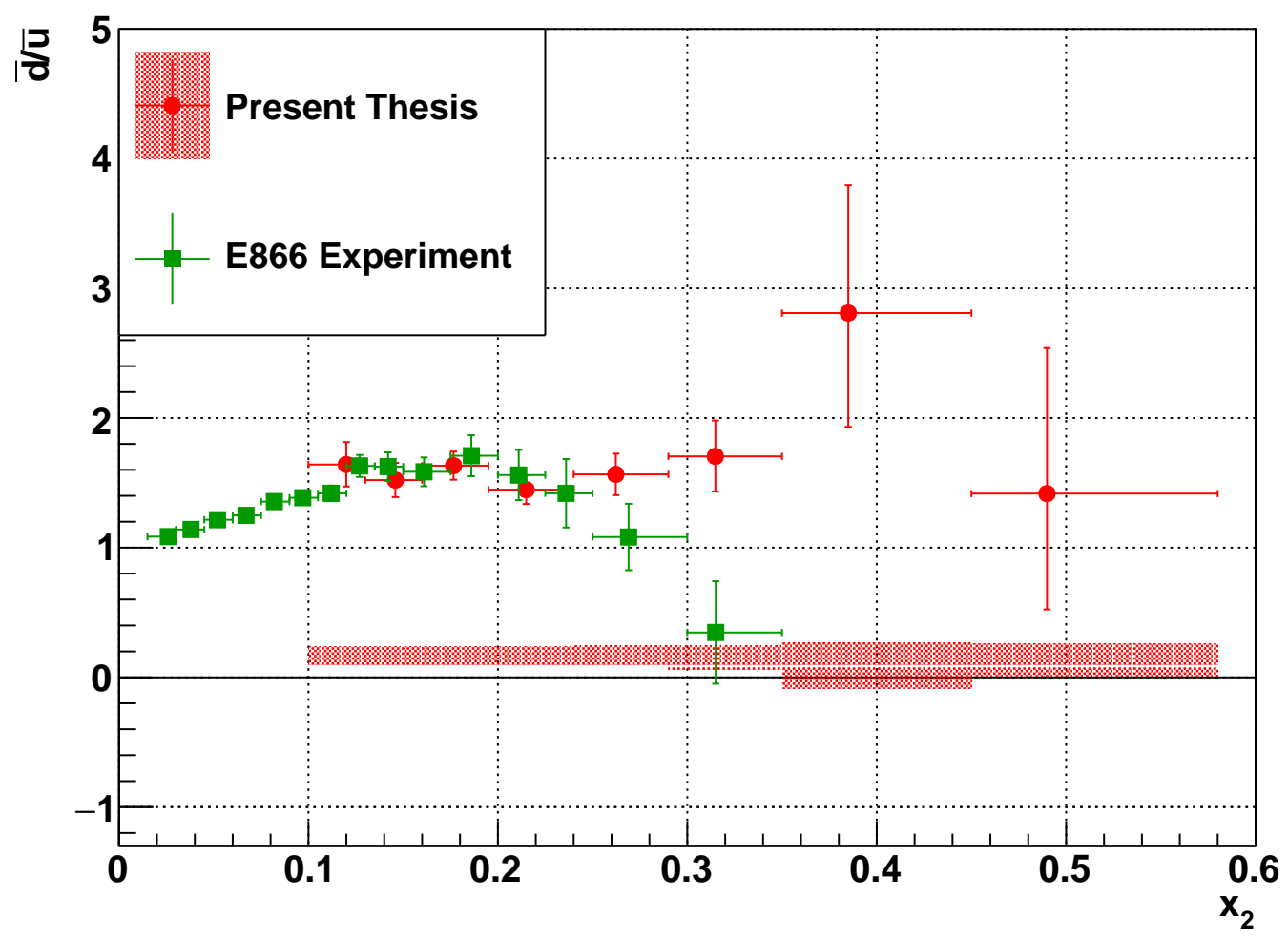

Figure 6.2: Comparison of the results in present thesis and those by the E866 experiment. The red points are the result in the present thesis. The green points are the results from the E866 experiment.

$x<0.24$ are consistent. The statistics at $x \sim 0.3$ in the present result are larger than those of the E866 experiment. The value of $\bar{d} / \bar{u}$ at $x \sim 0.3$ is about 1.5 as well as the results at lower $x$ regions although the result of E866 experiment is less than 1.0 with large statistical error. The impact of the result is that the flavor asymmetry $\bar{d} / \bar{u}$ keeps around 1.5 from $0.1<x<0.35$. The results at $0.35<x<0.58$ are also consistent with 1.5 due to the large statistical errors.

\subsection{Origin of Flavor Asymmetry}

The theme of the present research is the hypothesis of the flavor symmetry. Experimental evidence of the flavor symmetry has not been found. It had been assumed based on QCD. In QCD, the quarkantiquark pairs are created from the gluon splitting. The masses of $u$ and $d$ quarks are small, thus the gluon splitting is expected to be independent of these quark flavor $(\bar{d}=\bar{u})$.

The NMC experiment showed the violation of the Gottfried sum rule [6]. It suggested the integrated $\bar{d}$ is more than integrated $\bar{u}$. The results of this thesis and E866 experiment [11] suggest that the $\bar{d}$ and $\bar{u}$ at each $x$ are also asymmetric. The result of this thesis suggests that the $\bar{d} / \bar{u}$ is always 
larger than 1.0 in the region of $0.1<x<0.58$ while the E866 experiment showed the $\bar{u}>\bar{d}$ at large $x$ with large uncertainty.

The possible causes of the asymmetry can be listed as follows:

- The gluon splitting depends on the flavor,

- Pauli blocking [47,48],

- Statistical model $[49,50]$, and

- Pion cloud model $[25,51]$.

\subsubsection{Pauli Blocking}

The Pauli blocking is based on the Pauli exclusion principle. The probability that the process $g \rightarrow u \bar{u}$ is suppressed compared to the process $g \rightarrow d \bar{d}$ since the proton consists of $u u d$ valence quarks. However, this effect was already found to be very small (less than 1\%) [48].

\subsubsection{Statistical Model}

The statistical model is also based on the Pauli exclusion principle. In this model, quarks are regarded as Fermi gas and gluons are regarded as Bose gas. They obey the Fermi-Dirac and Bose-Einstein functions, respectively. Figure 6.3 shows the comparison of the present thesis result and the statistical model. The points at $0.1<x<0.35$ are consistent. The points at $0.35<x<0.58$ are also consistent though the statistical error is large. More statistics is needed to clarify the $\bar{d} / \bar{u}$ behavior at $0.35<x<$ 0.58 . 


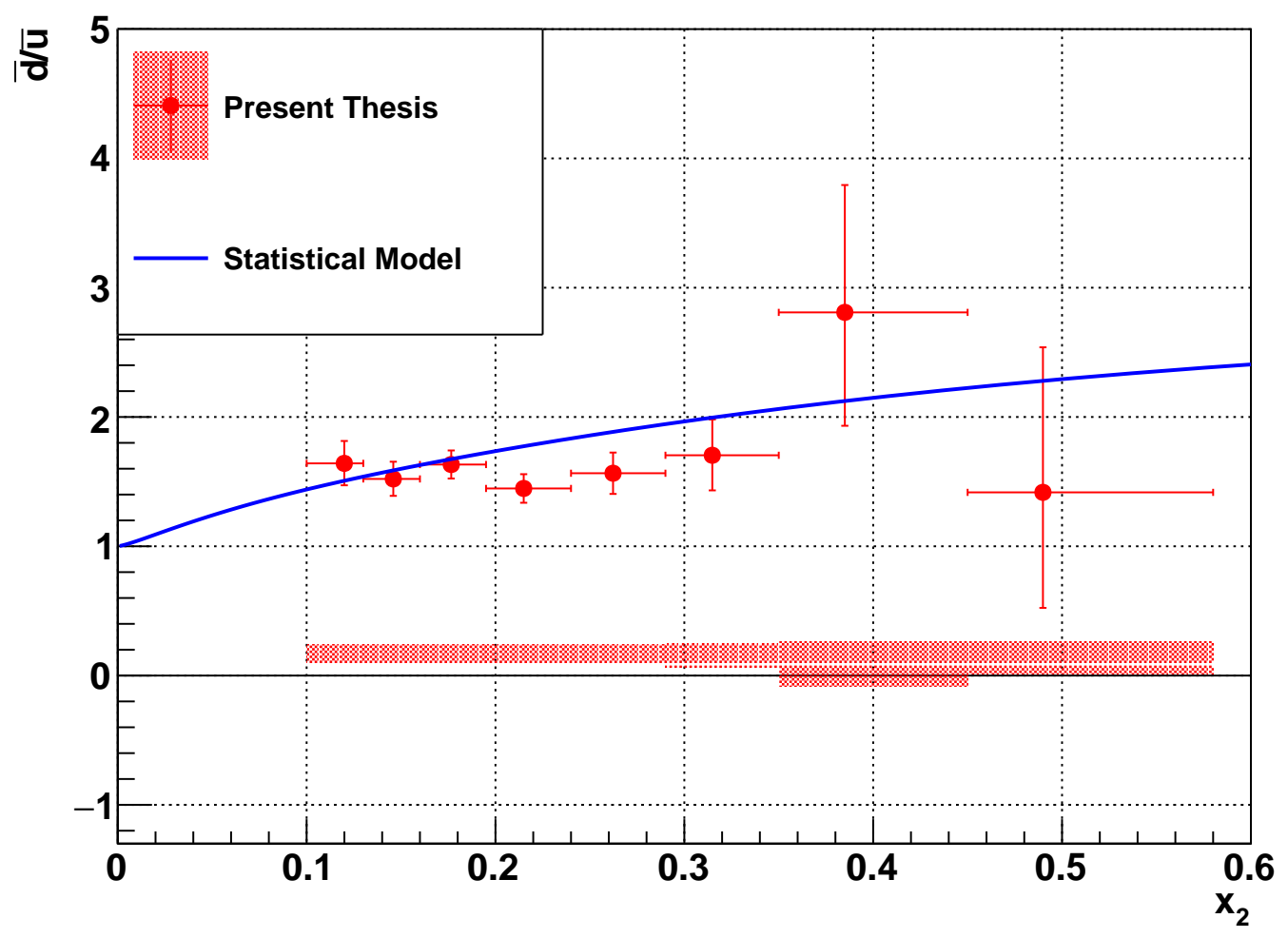

Figure 6.3: Comparison of the present result and the statistical model. The red points are the results in the present thesis. The blue line is the expected $\bar{d} / \bar{u}$ by the statistical model $[49,50]$. 


\subsubsection{Pion Cloud Model}

In the pion cloud model, the virtual pion contributes to the PDFs. The proton wave function is written as

$$
|p\rangle=(1-a-b)\left|p_{0}\right\rangle+a|N \pi\rangle+b|\Delta \pi\rangle
$$

Since the constituent $u$-quark is more than the constituent d-quark in the proton, the process $|p\rangle \rightarrow$ $\left|n \pi^{+}\right\rangle$by creating $d \bar{d}$ pair is more likely than the process $|p\rangle \rightarrow\left|\Delta^{++} \pi^{-}\right\rangle$by creating $u \bar{u}$ pair. This results in $\bar{d}>\bar{u}$. Figure 6.4 shows the comparison of the present result and the pion cloud model. The

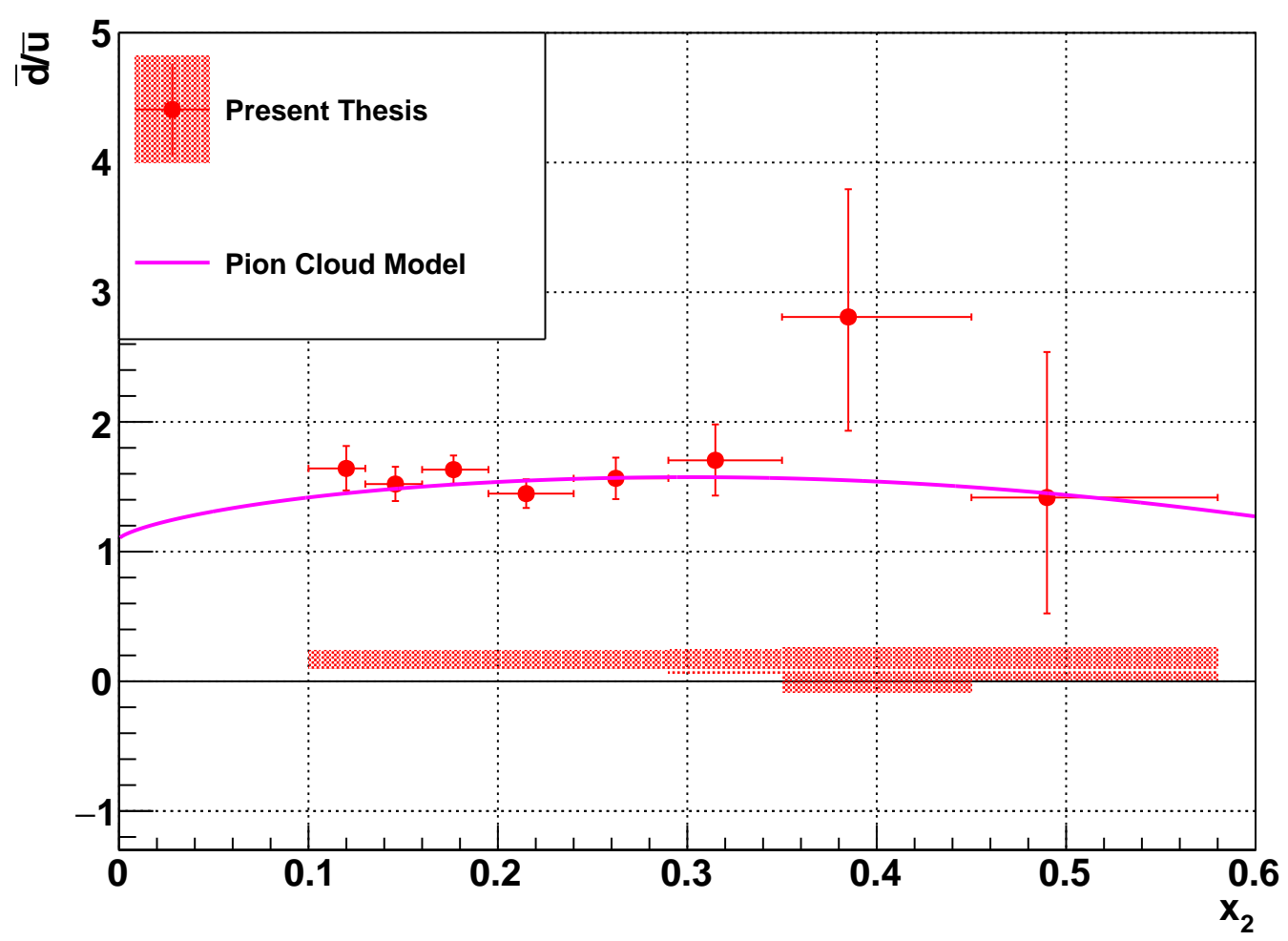

Figure 6.4: Comparison of the present thesis result and the pion cloud model. The red points are the results in the present thesis. The purple line is the expected $\bar{d} / \bar{u}$ by the pion cloud model [25].

results are consistent with the pion cloud model. The point at the seventh bin is slightly far from the curve of pion cloud model, but is consistent due to the large statistical error. Currently, both of the statistical model and the pion cloud model are consistent with the present results. 


\section{Chapter 7}

\section{Summary and Conclusion}

\section{Summary and Conclusion}

The SeaQuest experiment is a Drell-Yan experiment performed at Fermilab. The purpose of the SeaQuest is to determine the flavor asymmetry of antiquarks as a function of Bjorken $x$ in the range $0.1<x<0.45$.

The SeaQuest spectrometer measures the muon pairs in the final state of the Drell-Yan process in the $p-p$ and $p-d$ reactions. SeaQuest uses a $120 \mathrm{GeV}$ proton beam provided by the Fermi Main Injector and $\mathrm{LH}_{2}$ and $\mathrm{LD}_{2}$ targets. In the Drell-Yan process of proton-proton reaction, an antiquark in a proton and a quark in another proton annihilate and create a virtual photon, which then decays into a muon pair $\left(q \bar{q} \rightarrow \gamma^{*} \rightarrow \mu^{+} \mu^{-}\right)$. This process is suitable for investigating the antiquarks because an antiquark is always involved in the process. Bjorken $x$ dependence of flavor asymmetry of antiquarks, $\bar{d} / \bar{u}$, is important to understand the structure of the nucleon. From technical point of view, in exerimental with high intensity beam, it is important to control the tracking efficiency of charged particles.

The following points can be listed as the summary of this thesis.

- A new analysis method is developed and used to obtain the cross section ratio: the tracking efficiency is corrected for the beam intensity dependence by the occupancy-based method. As the result, nearby all the available data became analyzable. The amount of analyzable data increased by about $40 \%$ compare to the previous analysis.

- The cross section ratio of $\sigma^{p d} / 2 \sigma^{p p}$ for $0.1<x<0.58$ is obtained. This range is wider than in the proposal of SeaQuest. Cross section at each bin is larger than 1.0. The highest $x$ point is consistent with 1.0 within the statistical error.

- The flavor asymmetry of antiquark $\bar{d} / \bar{u}$ at $0.1<x<0.58$ is obtained. The $\bar{d} / \bar{u}$ at each bin is larger than 1.0. The highest $x$ point is consistent with 1.0 within the statistical error.

- The extracted $\bar{d} / \bar{u}$ can be used to investigate the validity of the theoretical models and is useful for understanding the structure of the nucleon.

- The flavor symmetry in the basic process is the fundamental feature of QCD. The present result raises a question on it. The higher order process involving multi-partons may generate the 
observed flavor asymmetry. It is now quite important to clarify these effects theoretically.

The present result has a practical application as well. Antiquark distributions are important as inputs to simulations of hadron reactions such as $W^{ \pm}$production in various experiments. The new knowledge on antiquark distributions helps to improve the precision of the simulations.

\section{Outlook}

The SeaQuest is currently taking data in Run 5. By the end of Run 5, the number of protons on target will increase by about $90 \%$ compared to the protons used in this thesis analysis. The SeaQuest may continue further data taking after Run 5. The SeaQuest data will provide most precise ratio $\bar{d} / \bar{u}$ in the high and wide $x_{2}$ range. 


\section{Acknowledgments}

I would like to express my deep gratitude to my supervisor Prof. Toshi-Aki Shibata. His guidance on the detector construction and the data analysis, and his advice on my doctor thesis were essential. He provided me with the opportunity to participate in the SeaQuest experiment at Fermi National Accelerator Laboratory and to study at the frontier of the particle physics. It was a great experience for me.

I am also deeply grateful to Prof. Kenichi Nakano for his valuable help and discussions. His advice concerning both hardware and software was very thoughtful.

I would like to thank all the Japanese group of the SeaQuest: Dr. Yuji Goto, Prof. Shin'ya Sawada, Prof. Yoshiyuki Miyachi, and Dr. Takahiro Sawada. The discussion with them at the Japanese group meeting was greatly helpful and valuable.

Dr. Paul E. Reimer and Dr. Don Geesaman, who is co-spokes-persons, gave me an opportunity to work in the SeaQuest experiment and gave me advice constantly. Dr. Dave C. Christian supported me during my stay at the Fermilab. Dr. Chuck N. Brown advised me on running the drift chamber and on the analysis. I would like to thank all of them.

I would like to thank the members of the $\bar{d} / \bar{u}$ analysis group, Prof. Kenichi Nakano, Dr. Markus Diefenthaler, Dr. Shou Miyasaka, Bryan Kerns, Shivangi Prasad, Arun Tadepalli, Dr. Paul E. Reimer, and Prof. Naomi Makins. The discussion with them is very useful and helpful for my analysis.

I always discussed the analysis with Dr. Kun Liu, Po-Ju Lin, Dr. Shou Miyasaka and Arun Tadepalli during my stay at Fermilab. The discussion with them advanced my research greatly. Especially, Dr. Shou Miyasaka always advised me kindly.

I like to thank all the students in the SeaQuest Japan group and Shibata Lab: Shou Miyasaka, Rui Sanada, Shumpei Nara, Takuto Miyazaki, Wataru Saito, Suguru Tamamushi, Yasufumi Kunisada, Koji Igarashi, Yuki Fujii, and Haruka Yamada.

I would like to thank all the members of the SeaQuest. It was a great pleasure for me to work in SeaQuest.

Lastly, I would like to thank my parents for supporting me many years. 


\section{References}

[1] B. Povh, K. Rith, C. Scholz, F. Ztsche, "Particles and Nuclei: An Introduction to the Physical Concepts", Springer, 1993.

[2] S. Kumano, "Nuclear Physics", KEK, 2016 (written in Japanese)

[3] E. D. Bloom et al., "High-Energy Inelastic $e-p$ Scattering at $6^{\circ}$ and $10^{\circ}$, Phys. Rev. Lett., Vol. 23, pp.930-934, Oct 1969.

[4] M. Breidenbach et al., "Observed Behavior of Highly Inelastic Electron-Proton Scattering", Phys. Rev. Lett., Vol. 23, pp. 935-939, Oct 1969.

[5] D. P. Barber et al., "Discovery of Three-Jet Events and a Test of Quantum Chromodynamics at PETRA", Phys. Rev. Lett., 43, p. 830, Sept 1979.

[6] P. Amaudruz et al., "Gottfried Sum from the Ratio $F_{2}^{n} / F_{2}^{p}$ ", Phys. Rev. Lett., Vol. 66, pp. 27122715, May 1991.

[7] M. Arneodo et al., "Reevaluation of the Gottfried sum", Phys. Rev. D., Vol. 50, pp. R1-R3, jul 1994.

[8] A. Baldit et al., "Study of the isospin symmetry breaking in the light quark sea of the nucleon from the Drell-Yan process", Phys. Lett. B, Vol. 332, pp. 244-250, 1994.

[9] R. S. Towell et al., "Improved measurement of the $\bar{d} / \bar{u}$ asymmetry in the nucleon sea", Phys. Rev. D, Vol. 64, p. 052002, Aug 2001.

[10] E.A. Hawker et al., "Measurement of the Light Antiquark Flavor Asymmetry in the Nucleon Sea", Phys. Rev. Lett., 80, p. 3715. 1998.

[11] G.T. Garvey and J.-C. Peng, "Flavor asymmetry of light quarks in the nucleon sea", Prg. Part. Nucl. Phys., Vol. 47, No. 1, pp. 203-243, 2001

[12] H. W. Kendall, "Deep inelastic scattering: Experiments on the proton and the observation of scaling”, Rev. Mod. Phys., Vol. 63, pp. 597-614, Jul 1991.

[13] D. J. Gross and F. Wilczek, "Ultraviolet Behavior of Non-Abelian Gauge Theories", Phys. Rev. Lett., Vol. 30, 1343, Jun 1973. 
[14] L.D. Faddeev and V.N. Popov, "Feynman diagrams for the Yang-Mills field", Phys. Rev. B, Vol. 25, pp. 29-30, July 1967.

[15] R. P. Feynman, "Very High-Energy Collisions of Hadrons", Phys. Rev. Lett., Vol. 23, pp. 14151417, Dec 1969.

[16] M. Gell-Mann, “A schematic model of baryons and mesons”, Phys. Lett., Vol. 8, pp. 214-215, Feb 1964.

[17] S. D. Drell and T. M. Yan, "Massive Lepton-Pair Production in Hadron-Hadron Collisions at High Energies", Phys. Rev. Lett, Vol. 25, p. 316, Aug 1970.

[18] A. D. Martin, W. J. Stirling, R. S. Thorne and G. Watt, "Parton distributions for the LHC", Eur. Phys. J. C, Vol. 63, No. 2, pp. 189-285, 2009.

[19] K. Gottfried, "Sum Rule for High-Energy Electron-Proton Scattering”, Phys. Rev. Lett., Vol. 18, pp. 1174-1177, Jun 1967.

[20] S. Stein et al., "Electron scattering at $4^{\circ}$ with energies of 4.5-20 GeV", Phys. Rev. D, Vol. 12, pp. 1884-1919, Oct 1975.

[21] A. Bodek et al., "Comparisons of Deep-Inelastic $e-p$ and $e-n$ Cross Sections", Phys. Rev. Lett., Vol. 30, pp. 1087-1091, May 1973.

[22] J.J. Aubert et al., "The ratio of the nucleon structure functions $F_{2}^{N}$ for iron and deuterium", Phys. Lett. B, Vol. 123, No. 3, pp. 275-278, 1983.

[23] J.J. Aubert et al., "Measurements of the nucleon structure functions $F_{2}^{N}$ in deep inelastic muon scattering from deuterium and comparison with those from hydrogen and iron", Nucl. Phys. B, Vol. 293, pp. 740-786, 1987.

[24] D. Allasia et al., "Measurement of the neutron and the proton $F_{2}$ structure function ratio", Phys. Lett. B, Vol. 249, No. 2, pp. 366-372, 1990.

[25] J. C. Peng et al., "“ $\bar{d} / \bar{u}$ asymmetry and the origin of the nucleon sea", Phys. Rev. D, Vol. 58, p. 092004, Sep 1998.

[26] S. Kumano, "Flavor asymmetry of antiquark distributions in the nucleon", Phys. Rep., Vol. 303, No. 4, pp. 183-257, 1998.

[27] H. L. Lai et al., "Improved parton distributions from global analysis of recent deep inelastic scattering and inclusive jet data", Phys. Rev. D, Vol. 55, pp. 1280-1296, Feb 1997.

[28] H. L. Lai et al., "Global QCD analysis of parton structure of the nucleon: CTEQ5 parton distributions", Eur. Phys. J. C, Vol. 12, No. 3, pp. 375-392, 2000. 
[29] J. Arrington et al., "Drell-Yan Measurement of Nucleon and Nuclear Structure with the Fermilab Main Injector: E906”, SeaQuest proposal, 2006.

[30] “The Fermilab Main Injector Technical Design Handbook”, Technical report, Fermi National Accelarator Laboratory, 1994.

[31] Private discussion with Tom O’Conner.

[32] "Garfield - simulation of gaseous detectors.", http://garfield.web.cern.ch/garfield/.

[33] K. Nagai, “Chamber Efficiency in Run2”, SEAQUEST Document 1321-v1, July 2015.

[34] K. Nagai, “Correction of Chamber Plane Resolution”, SEAQUEST Document 1686-v2, June 2016.

[35] W. M. Bokhari, J. G. Heinrich, N.S. Lockyer, and F. M. Newcomer, "The ASDQ ASIC [Centeral Outer Tracker CDF uprade application]”, pp. 445-446, Institute of Electrical and Electronics Engineers, 1998.

[36] "VME bus architecture", http://www. caen.it/csite/CaenProd.jsp?parent=11\&idmod= 705.

[37] S.-H. Shiu et al., "FPGA-based trigger system for the Fermilab SeaQuest experiment", NIMinPhysicsResearchSectionA, Vol. 802, pp. 82-88, 2015.

[38] "Data Acquisition Support Group at Thomas Jefferson National Accelerator Facility", https: //coda.jlab.org/drupal/.

[39] "A toolkit for the simulation of the passage of particles through matter", http://geant4 . web. cern.ch/geant4/.

[40] M. H. Schub et al., "Measurement of $J / \psi$ and $\psi^{\prime}$ production in $800 \mathrm{GeV} / c$ proton-gold collisions", Phys. Rev. D, Vol. 52, p. 1307, 1995.

[41] J. Pumplin et al., "New Generation of Parton Distributions with Uncertainties from Global QCD Analysis", JHEP, Vol. 012, p. 0207, 2002.

[42] K. Nagai, “Hit Cluster Removal”, SEAQUEST Document 1123-v1, Aug 2014.

[43] K. Nagai et al., "Progress of Drell-Yan experiment by SeaQuest at Fermilab", RIKEN Accelerator Progress Report, Vol. 49. 2016.

[44] "Atomic and Nuclear Properties of Materials for more than 300 materials", http://pdg. 1 bl. gov/2014/AtomicNuclearProperties/

[45] H. Lai et al., "New parton distributions for collider physics", Phys. Rev. D, Vol. 82, p. 074024, 2010. 
[46] S. Miyasaka, "Probing Flavor Asymmetry of Anti-quarks in the Proton by Drell-Yan Experiment SeaQuest”, PhD thesis, Tokyo Institute of Technology, 2016.

[47] R. D. Field, and R. P. Feynman, "Quark elastic scattering as a source of high-transversemomentum mesons”, Phys. Rev. D, Vol. 15, pp. 2590-2616, May 1977.

[48] D.A. Ross and C.T. Sachrajda, "Flavour symmetry breaking in antiquark distributions", Nucl. Phys. B, Vol. 149, No. 3, pp. 497-516, 1979.

[49] E. Basso, C. Bourrely, R. Pasechnik and J. Soffer, "The DrellYan process as a testing ground for parton distributions up to LHC", Nucl. Phys. A, Vol. 948, pp. 63-77, 2016.

[50] C. Bourrely and J. Soffer, "New developments in the statistical approach of parton distributions: Tests and predictions up to LHC energies", Nucl. Phys. A, Vol. 941, pp. 307-334, 2015.

[51] S. Kumano, "Effects of $\pi N N$ form factor on pionic contributions to $\bar{d}(x)-\bar{u}(x)$ distribution in the nucleon", Vol. 43, p. 3067, May 1991 


\section{List of Figures}

1.1 Proton structure in different energy scales. . . . . . . . . . . . . . 4

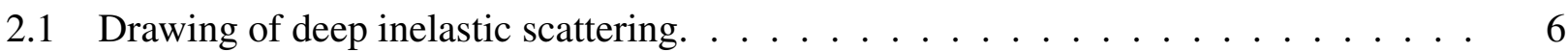

2.2 NLO PDF calculation at $Q^{2}=10 \mathrm{GeV}^{2}$ and $Q^{2}=10^{4} \mathrm{GeV}^{2} \ldots \ldots \ldots$

2.3 Drawing of Drell-Yan process. . . . . . . . . . . . . . . . 10

2.4 Acceptance of the SeaQuest spectrometer. . . . . . . . . . . . . . . 11

2.5 NMC results of $F_{2}^{p}(x)-F_{2}^{n}(x)$ and $\int_{x}^{1}\left(F_{2}^{p}(x)-F_{2}^{n}(x)\right) d x / x \ldots \ldots \ldots$

2.6 Results of E866 experiment and NA51 experiment. . . . . . . . . . . . . . . . 16

2.7 Comparison of the $\bar{d} / \bar{u}$ distribution in CTEQ4M and CTEQ5M. . . . . . . . . . . 16

3.1 Integrated protons on target. . . . . . . . . . . . . . . . . 19

3.2 Drawing of Fermilab Main Injector and SeaQuest experimental hall. . . . . . . . . 20

3.3 Beam structure of Fermilab Main Injector. . . . . . . . . . . . . . . . . . . 20

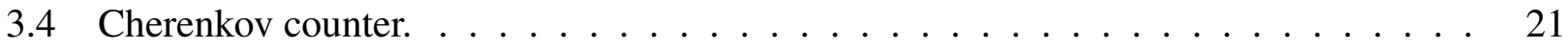

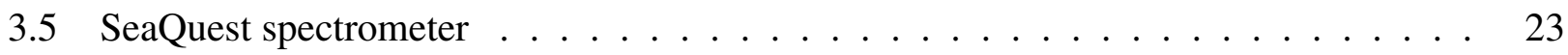

3.6 Targets used in the SeaQuest spectrometer. . . . . . . . . . . . . . . . . . 24

3.7 Drawing of the first dipole magnet FMag. . . . . . . . . . . . . . . . 26

3.8 Top view of the detector and muon pair tracks. . . . . . . . . . . . . . . . 27

3.9 Drawing of the second dipole magnet KMag. . . . . . . . . . . . . . . . . 28

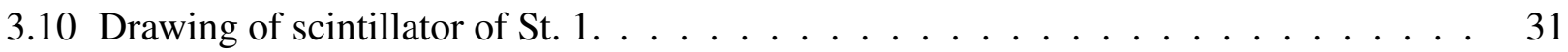

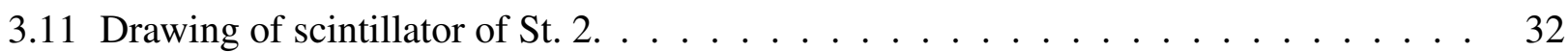

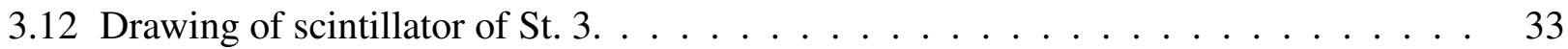

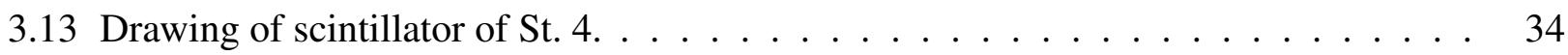

3.14 Structure of St. 1 drift chamber. . . . . . . . . . . . . . . . . . . 35

3.15 Structure of St. 2 drift chamber. . . . . . . . . . . . . . . . . . . . 37

3.16 Structure of St. 3+ drift chamber. . . . . . . . . . . . . . . . . . . . 37

3.17 Structure of St. 3- drift chamber. . . . . . . . . . . . . . . . . . 38

3.18 Cell structure of St. 3 drift chamber. . . . . . . . . . . . . . . . . . . . . 39

3.19 Electric potential between sense wire and field or cathode wires. . . . . . . . . . . 40

3.20 RT-curve of U plane on D3m. . . . . . . . . . . . . . . . . . . . 41

3.21 Residual at each plane. . . . . . . . . . . . . . . . . . . . 44

3.22 Top view and side view of the proportional tubes. . . . . . . . . . . . . . . 47

3.23 Structure of the proportional tubes. . . . . . . . . . . . . . . . . . . 48 
3.24 Drawing of the readout system. . . . . . . . . . . . . . . . . . 48

3.25 ASDQ card . . . . . . . . . . . . . . . . . . . . . . . 49

3.26 Level Shifter Board. . . . . . . . . . . . . . . . . . . . . . . . 49

3.27 Visualization of fired trigger-roads. . . . . . . . . . . . . . . . . 52

3.28 A chain of VME crates. . . . . . . . . . . . . . . . . . . . 54

3.29 Acceptance of " $4 \pi$ " GMC and "accepted" GMC . . . . . . . . . . . . . . 58

4.1 Procedure of the dimuon reconstruction. . . . . . . . . . . . . . . . . 59

4.2 Number of hits in the St. 1 drift chamber. . . . . . . . . . . . . . 61

4.3 Drawing on the method of checking if the true hits are removed by the hit removal. . $\quad 62$

4.4 Distributions of the occupancy on St. 3+ drift chamber before and after the track

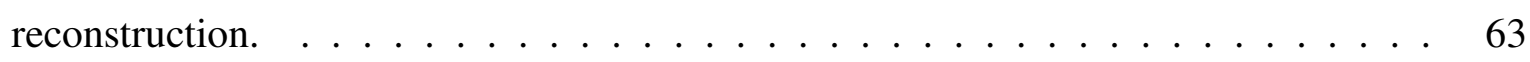

4.5 Rough structure of drift chamber. . . . . . . . . . . . . . . . . . . . 64

4.6 Components of $u_{\text {win }} \ldots \ldots \ldots \ldots \ldots \ldots$

4.7 Accepted range of $\mathrm{U}$ wires. . . . . . . . . . . . . . . . . 66

4.8 Calculation of $u$ hit position based on $x$ hit position. . . . . . . . . . . . 67

4.9 Definition of sagitta on St. $1(\mathrm{~S} 1)$ and St. $2(\mathrm{~S} 2) \ldots \ldots \ldots 9$

4.10 Vertex reconstruction at FMag slice. . . . . . . . . . . . . . . 71

4.11 Muon momentum variation due to the energy loss at FMag. . . . . . . . . . . . 72

4.12 Flow chart of selections and quality cuts. . . . . . . . . . . . . . . 74

4.13 Target position as a function of spill ID. . . . . . . . . . . . . 76

4.14 Duty factor as a function of spill ID. . . . . . . . . . . . . . . 77

$4.15 \mathrm{G} 2 \mathrm{SEM}$ as a function of spill ID. . . . . . . . . . . . . . . . 78

4.16 QIEsum as a function of spill ID . . . . . . . . . . . . . . . . . . . . . . . . . . . . . . . .

4.17 Flow chart of data taking in one spill. . . . . . . . . . . . . . . 81

4.18 TSGo as a function of spill ID. . . . . . . . . . . . . . . . . 82

4.19 AfterInhFPGA1 as a function of spill ID. . . . . . . . . . . . . 83

4.20 AcceptedFGPA1 as a function of spill ID. . . . . . . . . . . . . . . 84

4.21 AcceptedFGPA1/AfterInhFPGA1 as a function of spill ID. . . . . . . . . . 85

4.22 Inhibit as a function of spill ID . . . . . . . . . . . . . . . . . . . . . . . . . . .

4.23 Busy as a function of spill ID. . . . . . . . . . . . . . . . . 87

4.24 Number of track associated hits. . . . . . . . . . . . . . . . . . . 89

4.25 Reduced $\chi^{2} \ldots \ldots \ldots \ldots \ldots \ldots$

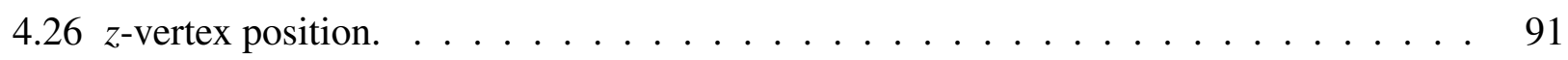

$4.27 z$-momentum at St. 1. . . . . . . . . . . . . . . . . . . 92

$4.28 z$-momentum at St. 1 with selections of "Number of track associated hits=15" and dimuon mass $>4.2 \mathrm{GeV} \ldots \ldots \ldots \ldots . \ldots \ldots$

$4.29 z$-vertex position of Monte-Carlo data . . . . . . . . . . . . . . . . . . . . . . . . . . . . . . . . . .

$4.30 z$-momentum at St. 1 of Monte-Carlo data. . . . . . . . . . . . . . . . . 95

4.31 Vertex position. . . . . . . . . . . . . . . . . . . 96

4.32 Vertex position of Monte-Carlo data. . . . . . . . . . . . . . . 97 
4.33 Momentum at vertex position. . . . . . . . . . . . . . . . . . . 97

4.34 Momentum at vertex position of Monte-Carlo data. . . . . . . . . . . . . . 97

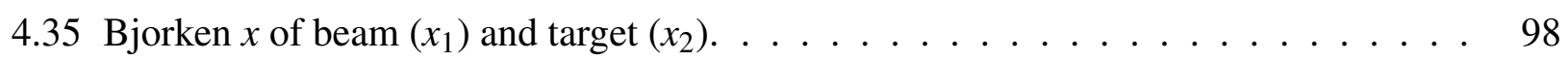

4.36 Bjorken $x$ of beam $\left(x_{1}\right)$ and target $\left(x_{2}\right)$ of Monte-Carlo data. . . . . . . . . . . 98

4.37 Feynman $x . \ldots \ldots \ldots \ldots \ldots$

4.38 Feynman $x$ of Monte-Carlo data. . . . . . . . . . . . . . . . . . . 99

4.39 Difference between $z$-vertex positions of $\mu^{+}$and $\mu^{-} \ldots \ldots \ldots$. . . . . . . 100

$4.40 \chi^{2}$ of vertex reconstruction. . . . . . . . . . . . . . . . 101

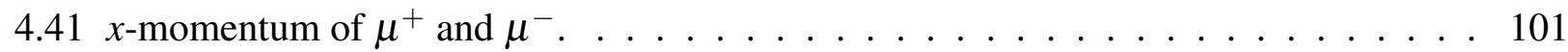

$4.42 x$-momentum of $\mu^{+}$and $\mu^{-}$of Monte-Carlo data. . . . . . . . . . . . 102

4.43 Dimuon mass. . . . . . . . . . . . . . . . . . . . . 102

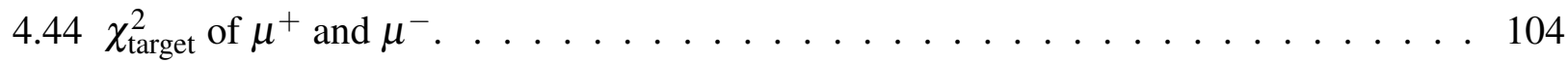



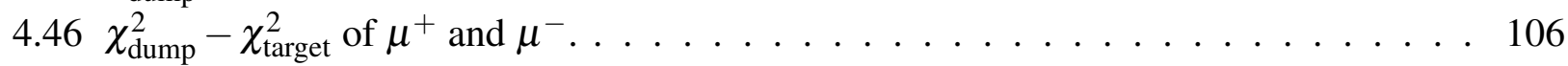

$4.47 z$-vertex position. . . . . . . . . . . . . . . . 107

$4.48 z$-vertex distribution of muon pairs whose invariant masses are larger than $4.2 \mathrm{GeV} / c^{2} .108$

4.49 Flow chart of obtaining the dimuon corrected yields. . . . . . . . . . . . . 110

4.50 Tracking efficiency as a function of occupancy. . . . . . . . . . . . . . 111

4.51 Occupancy dependence of the tracking efficiency and the fitting result at each $x_{2}$. . . 112

4.52 Tracking efficiency as a function of occupancy at each $x_{2} \ldots \ldots \ldots 113$

4.53 Tracking efficiencies with errors at each $x_{2}$ bin. . . . . . . . . . . . . 115

4.54 Track hit position of $\mu^{-}$at St. 1 drift chamber. . . . . . . . . . . . . . . 116

4.55 Hit distribution at St. 1 drift chamber. . . . . . . . . . . . . . . . 117

4.56 Relation between the occupancy and $x_{2} \ldots \ldots \ldots \ldots \ldots$. . . . . . . . . . . . . . . . . . . . . . .

4.57 Dimuon yields of $\mathrm{LH}_{2}, \mathrm{LD}_{2}$ and empty target. . . . . . . . . . . . . . . . 119

4.58 Dimuon yield ratio of $\mathrm{LD}_{2}$ to $\mathrm{LH}_{2}$ as a function of the occupancy after the tracking efficiency correction and the empty target subtraction. . . . . . . . . . . . 120

4.59 Dimuon yield ratio of $\mathrm{LD}_{2}$ to $\mathrm{LH}_{2}$ as a function of the occupancy range. . . . . . . 121

4.60 Dimuon yield ratio as a function of occupancy range in each $x_{2}$ bin. . . . . . . . 122

4.61 Mass distribution of $\mathrm{LH}_{2}$ target events in each occupancy bin. . . . . . . . . . . . . 124

4.62 Mass distribution of $\mathrm{LD}_{2}$ target events in each occupancy bin. . . . . . . . . . . 125

4.63 Ratio of the combinatorial background to the Drell-Yan. . . . . . . . . . . . 126

4.64 Occupancy distribution of dimuons used in the analysis. . . . . . . . . . . . . . . 129

4.65 Fitting result of occupancy dependence of dimuon yield ratio. . . . . . . . . . . 130

4.66 Dimuon yield ratio of $\mathrm{LH}_{2}$ and $\mathrm{LD}_{2}$ with cut-off mass of $4.2 \mathrm{GeV}$ and $4.5 \mathrm{GeV}$. . . 131

4.67 Cross section ratio as a function of $x_{2} \ldots \ldots \ldots \ldots$

5.1 Flow chart of the iterative analysis for extracting $\bar{d} / \bar{u} \ldots \ldots \ldots \ldots$

5.2 The result of the ratio $\bar{d} / \bar{u} \ldots \ldots \ldots \ldots \ldots \ldots \ldots$

6.1 Comparison of results in present thesis and those by Miyasaka. . . . . . . . . . . . . 144 
6.2 Comparison of the results in present thesis and those by the E866 experiment. . . . . 145

6.3 Comparison of the present thesis result and the statistical model. . . . . . . . . . 147

6.4 Comparison of the present thesis result and the pion cloud model. . . . . . . . . 148 


\section{List of Tables}

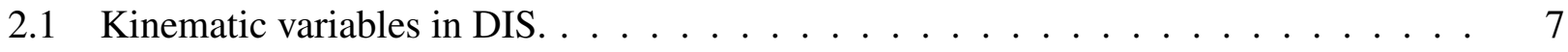

3.1 Timeline of SeaQuest experiment. . . . . . . . . . . . . . . . . . . . 19

3.2 Types and characteristics of the SeaQuest targets. . . . . . . . . . . . . . 25

3.3 Number and size of scintillator at each hodoscope plane. . . . . . . . . . . . . . . 29

3.4 Parameters of all the drift chambers. . . . . . . . . . . . . . . . . 36

3.5 List of the dead or noisy wire position. . . . . . . . . . . . . . . . . . . . . . . . . . .

3.6 Plane detection efficiency of each drift chamber plane. . . . . . . . . . . . . . . 43

3.7 Residual and plane position resolution. . . . . . . . . . . . . . . . . 45

3.8 Specification of proportional tubes. . . . . . . . . . . . . . . . 46

3.9 Name and characteristics of each trigger. . . . . . . . . . . . . . . . . 50

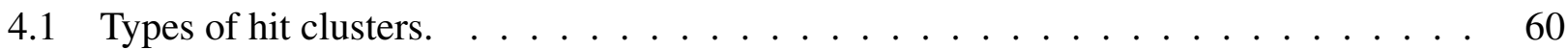

4.2 Upper limits of the occupancy of each drift chamber. . . . . . . . . . . . . . 63

4.3 Constants used in vertex fitting. . . . . . . . . . . . . . . 70

4.4 Spill level selections. . . . . . . . . . . . . . . . . . . . 75

4.5 The number of good spills and the number of live protons in each roadset. . . . . . 75

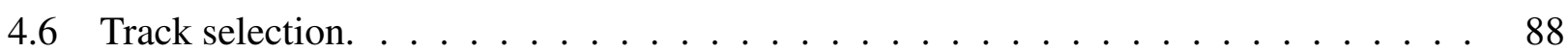

4.7 Dimuon selection. . . . . . . . . . . . . . . . . . . . 96

4.8 Target-Dump Separation . . . . . . . . . . . . . . . . . 103

4.9 Dimuon raw yields of $\mathrm{LH}_{2}, \mathrm{LD}_{2}$ and Empty targets. . . . . . . . . . . . . . . 109

4.10 Fitting parameters at each $x_{2}$ bin. . . . . . . . . . . . . . . . . 113

4.11 Covariance matrix at each $x_{2}$ bin. . . . . . . . . . . . . . . . . 114

4.12 Dimuon corrected yields. . . . . . . . . . . . . . . . . . . . . 114

4.13 Values used in the analysis [44] . . . . . . . . . . . . . . . . 127

4.14 The purity of deuterium in $\mathrm{LD}_{2}$ target in each Roadset. . . . . . . . . . . . . . . 127

4.15 Fitting result of Fig. 4.65 . . . . . . . . . . . . . . . . . . . 130

4.16 Sources of systematic errors. . . . . . . . . . . . . . . . . . . 132

4.17 Cross section ratio and statistical and systematic errors at each $x_{2}$ bin. . . . . . . . 132

4.18 Breakdown of errors $(+1 \sigma$ shift $) \ldots \ldots \ldots \ldots \ldots$

4.19 Breakdown of errors $(-1 \sigma$ shift $) \ldots \ldots \ldots \ldots \ldots \ldots$

5.1 Statistical error matrix at each $i$ bin by upward shifting $j$ bin. . . . . . . . . . 138 
5.2 Statistical error matrix at each $i$ bin by downward shifting $j$ bin. . . . . . . . . . 139

5.3 Statistical error matrix at each $i$ bin by shifting $j$ bin. . . . . . . . . . . . . . 139

5.4 Breakdown of $\bar{d} / \bar{u}$ systematic errors. . . . . . . . . . . . . . . . . . 140

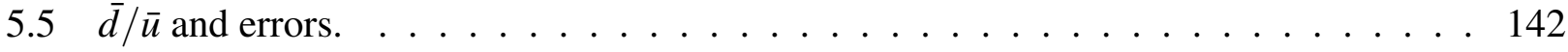

